\title{
Turbulence Hydrodynamics and Scalar Transport Processes in Boundary Layer Flow Past Corals
}

\author{
A Dissertation \\ Presented to \\ the faculty of the School of Engineering and Applied Science \\ University of Virginia
}

\author{
in partial fulfillment \\ of the requirements for the degree \\ Doctor of Philosophy \\ by \\ Jonathan Brady Stocking
}

May 2017 


\section{Copyright}

(C) 2017 Jonathan Stocking

All rights reserved. 


\title{
APPROVAL SHEET
}

\author{
This Dissertation \\ is submitted in partial fulfillment of the requirements \\ for the degree of \\ Doctor of Philosophy
}

Author Signature:

This dissertation has been read and approved by the examining committee:

$\begin{aligned} \text { Advisor: } & \text { Matthew Reidenbach } \\ \text { Committee Chair: } & \text { Haibo Dong } \\ \text { Committee Member: } & \text { Silvia Blemker } \\ \text { Committee Member: } & \text { Eric Loth } \\ \text { Committee Member: } & \text { Patricia Wiberg }\end{aligned}$

Accepted for the School of Engineering and Applied Science:

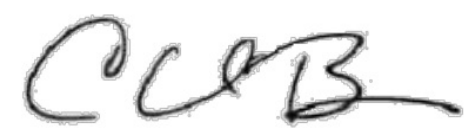

Craig H. Benson, School of Engineering and Applied Science

May 2017 


\section{Contents}

\begin{tabular}{ll}
\hline Abstract & ix
\end{tabular}

Nomenclature $\quad x i$

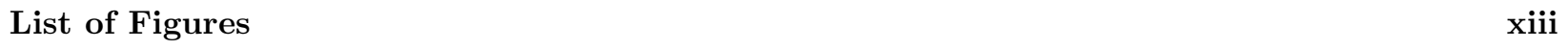

List of Tables $\quad x x$

\begin{tabular}{|l|l}
\hline Acknowledgments & xxi \\
\hline
\end{tabular}

\begin{tabular}{lll}
\hline & Introduction & 1
\end{tabular}

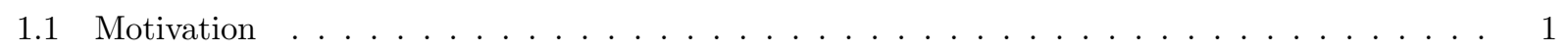

1.1.1 Economic, Environmental, and Cultural Value of Corals . . . . . . . . . . . . . 1

1.1.2 Mass Transfer and the Role of the Turbulent Boundary Layer . . . . . . . . . . . . . 2

1.2 Research Questions . . . . . . . . . . . . . . . . . . . . . . . . . . . 4

1.3 Contribution of Results $\ldots \ldots \ldots \ldots \ldots \ldots \ldots \ldots$

1.3 .1 Novelty and Significance $\ldots \ldots \ldots \ldots \ldots \ldots \ldots$

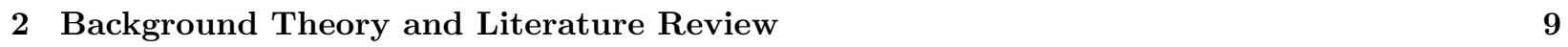

$2.1 \quad$ Coral Ecology $\ldots \ldots \ldots \ldots \ldots \ldots \ldots$

$2.1 .1 \quad$ Fundamentals of the Coral Lifecycle $\ldots \ldots \ldots \ldots \ldots$

$2.1 .2 \quad$ Benthic Boundary Layer and Concentration Gradients . . . . . . . . . . . . . . . . 11

2.1 .3 Turf-forming Macroalgae Degradation $\ldots \ldots \ldots \ldots \ldots \ldots$

2.2 Turbulent Boundary Layer Theory $\ldots \ldots \ldots \ldots$

$2.2 .1 \quad$ Equations of Motion $\ldots \ldots \ldots \ldots \ldots \ldots \ldots \ldots \ldots \ldots$

2.2 .2 Turbulent Kinetic Energy Budget $\ldots \ldots \ldots \ldots \ldots \ldots \ldots$

$2.2 .3 \quad$ Boundary Layer Scaling $\ldots \ldots \ldots \ldots$ 
2.3 Combined Wave-Current Flow . . . . . . . . . . . . . . . . . . . . . . . . . . . . . . . . . . . 17

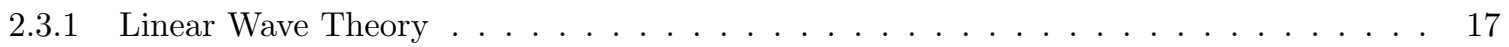

2.3 .2 Wave-Current Boundary Layers . . . . . . . . . . . . . . . . . . . . . . . . . . . . . . 18

2.4 Canopy Flow . . . . . . . . . . . . . . . . . . . . . . . . . . . . . 19

2.4 .1 Mixing Layer Analogy . . . . . . . . . . . . . . . . . . . . . . . . . . . . . . . 20

2.5 Large- and Small-Scale Morphology . . . . . . . . . . . . . . . . . . . . . . . . . . . . . . . . . 21

2.5 .1 Surface Roughness . . . . . . . . . . . . . . . . . . . . . . . . . . . . . . 22

2.5 .2 Roughness Length Scale and Roughness Function . . . . . . . . . . . . . . . . . . . . . 23

2.6 Mass Transfer . . . . . . . . . . . . . . . . . . . . . . . . . . . . . . . . . . . . . . . . . 24

3 Structure and Dynamics of Turbulent Boundary Layer Flow Over Healthy and Algae\begin{tabular}{ll} 
covered Corals & 27 \\
\hline
\end{tabular}

3.1 Introduction . . . . . . . . . . . . . . . . . . . . . . . . 27

3.1.1 Hydrodynamics of the Turbulent Boundary Layer . . . . . . . . . . . . . . . . . . . . . 28

$3.1 .2 \quad$ Boundary Layer Alterations Due to Waves and Canopies _ . . . . . . . . . . . . . . . . 29

3.2 Methods . . . . . . . . . . . . . . . . . . . . . . . . . . . 30

3.2.1 Study Site . . . . . . . . . . . . . . . . . . . . . . . . . . . . . 30

3.2 .2 Coral and Algal Morphology . . . . . . . . . . . . . . . . . . . . . . . . . . . . . . . . 31

3.2 .3 Instrumentation . . . . . . . . . . . . . . . . . . . . . . . . 32

$3.2 .4 \quad$ Analysis of Turbulence Statistics . . . . . . . . . . . . . . . . . . . . . . . . . . . . 34

3.3 Results . . . . . . . . . . . . . . . . . . . . . . . . . . 36

3.3 .1 Velocity Structure and Reynolds Stresses . . . . . . . . . . . . . . . . . . . . . . . . . 37

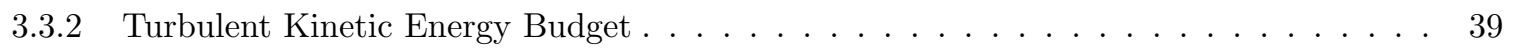

3.3.3 Bed Shear Stress . . . . . . . . . . . . . . . . . . . . . . . . . . . . . 40

3.3.4 $\quad$ PIV Spatial Analysis of Mean and Turbulent Flow Structure . . . . . . . . . . . . . . 41

3.4 Discussion . . . . . . . . . . . . . . . . . . . . . . . . . . . . . 43

3.4.1 Boundary Layer Characterization for Healthy Corals . . . . . . . . . . . . . . . . . . . 43

3.4 .2 Boundary Layer Characterization for Algae-covered Corals . . . . . . . . . . . . . . . 44

3.4 .3 Implications for Biology . . . . . . . . . . . . . . . . . . . . . . . . . . . 45

4 Experimental Investigation of Unidirectional Flow Past Smooth and Rough Hemispheres 47

4.1 Experimental Questions . . . . . . . . . . . . . . . . . . . . . . . . . . . . 48

4.2 Experimental Methods . . . . . . . . . . . . . . . . . . . . . . . . . 50

4.2 .1 Model Coral Geometries . . . . . . . . . . . . . . . . . . . . . 50 
$4.2 .2 \quad$ Water Tunnel Facilities and Model Setup . . . . . . . . . . . . . . . . . . . . 52

$4.2 .3 \quad$ Particle Image Velocimetry $\ldots \ldots \ldots \ldots \ldots \ldots$

4.2 .4 Dye Visualization Setup $\ldots \ldots \ldots \ldots \ldots$

4.3 Results: Mean Characteristics $\ldots \ldots \ldots \ldots \ldots \ldots \ldots$

4.3 .1 Flow Visualization $\ldots \ldots \ldots \ldots \ldots \ldots \ldots \ldots \ldots$

4.3 .2 Mean Horizontal Velocity $\ldots \ldots \ldots$. . . . . . . . . . . . . . . . . 59

4.3 .3 Polar Separation Angle $\ldots \ldots \ldots 1$

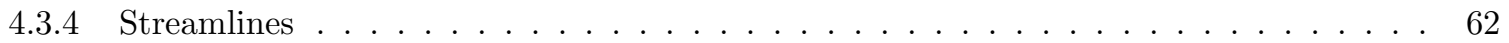

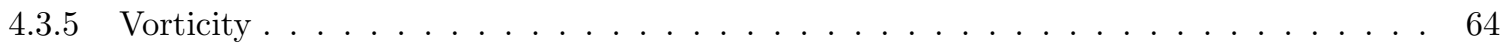

4.4 Results: Turbulence Characteristics . . . . . . . . . . . . . . . . . . . . . . 66

4.4 .1 Reynolds Shear Stress $\ldots \ldots \ldots$. . . . . . . . . . . . . . . . . . . 66

4.4 .2 Turbulence Production and Dissipation $\ldots \ldots \ldots \ldots \ldots$

4.4 .3 Joint Probability Distribution Functions $\ldots \ldots \ldots \ldots \ldots$

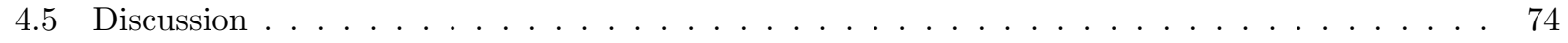

$4.5 .1 \quad$ Effect of Reynolds Number $\ldots \ldots \ldots \ldots \ldots \ldots$

4.5 .2 Effect of Roughness Type $\ldots \ldots \ldots \ldots \ldots$

4.6 Conclusions and Future Work $\ldots \ldots \ldots \ldots \ldots \ldots$

5 Large Eddy Simulations of Heat Transfer from Smooth and Rough Hemispheres under $\begin{array}{ll}\text { Unidirectional and Oscillatory Flow } & 87\end{array}$

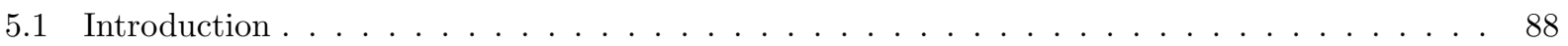

5.1 .1 Heat transfer fundamentals $\ldots \ldots \ldots \ldots \ldots \ldots$

5.1 .2 Convection heat transfer correlations $\ldots \ldots \ldots \ldots \ldots \ldots$

5.1 .3 Rough-wall heat transfer . . . . . . . . . . . . . . . . . . . . . . . . . 90

5.1 .4 Heat transfer for hemispheres $\ldots \ldots \ldots \ldots \ldots \ldots$. . . . . . . . . . . . 91

5.1 .5 Mass transfer for oscillatory flow $\ldots \ldots \ldots \ldots \ldots \ldots \ldots$

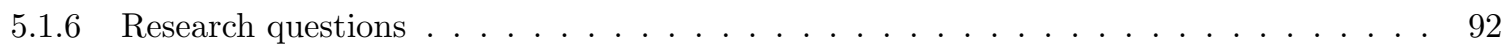

5.2 Formulation of the Numerical Model $\ldots \ldots \ldots \ldots$

5.2 .1 Domain setup and boundary conditions $\ldots \ldots \ldots \ldots \ldots \ldots$

$5.2 .2 \quad$ Large eddy simulation fundamentals $\ldots \ldots \ldots \ldots \ldots \ldots \ldots$

$5.2 .3 \quad$ LES governing equations and subgrid-scale model $\ldots \ldots \ldots \ldots$

$5.2 .4 \quad$ LES-Coupled heat transfer model $\ldots \ldots \ldots \ldots$. . . . . . . . . . . . . . 97

5.2 .5 Discretization and computational methods $\ldots \ldots \ldots \ldots \ldots \ldots$ 
5.3 Model Validation . . . . . . . . . . . . . . . . . . . . . . . . . 100

5.4 Numerical Results $\ldots \ldots \ldots \ldots$. . . . . . . . . . . . . . . . . . . . . . 104

5.4 .1 Results: Flow Characteristics . . . . . . . . . . . . . . . . . . . . . . . . 104

5.4 .2 Results: Heat Transfer Characteristics . . . . . . . . . . . . . . . . . . . . . . 116

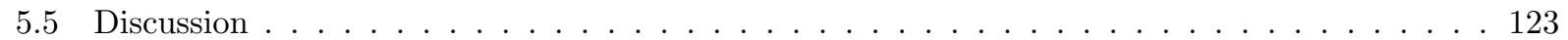

$5.5 .1 \quad$ Boundary layer and wake hydrodynamics $\ldots \ldots \ldots \ldots \ldots \ldots$

5.5 .2 Surface roughness and flow effects on convective heat transfer . . . . . . . . . . . 126

$5.5 .3 \quad$ Biological and ecosystem implications of heat and mass transfer $\ldots \ldots \ldots \ldots$

5.6 Conclusions $\ldots \ldots \ldots \ldots \ldots$

\begin{tabular}{lll}
\hline & Conclusions & 133
\end{tabular}

6.1 Relevance to Current Research in Coral Biology . . . . . . . . . . . . . . . . . . . . . 133

6.2 Relevance to Engineering Literature $\ldots \ldots \ldots \ldots \ldots$

\begin{tabular}{ll}
\hline Appendices & 139
\end{tabular}

\begin{tabular}{|l|l}
\hline Appendix A Appendix & 141
\end{tabular}

A.1 Wave-turbulence decomposition . . . . . . . . . . . . . . . . . . . . . . . . . 141

A.2 Turbulent kinetic energy (TKE) budget equations f . . . . . . . . . . . . . . . . . . 142

\begin{tabular}{ll}
\hline References & 157
\end{tabular} 


\section{Abstract}

Coral reefs provide an extensive range of ecosystem services to society, playing a critical role in shoreline protection, fishery habitat, tourism, and cultural/historic ties for local and indigenous communities. In addition to the inherent environmental significance of corals, these ecosystem services contribute billions of dollars annually in global economic value. Given the importance of corals, it is vital to understand the biological, ecological, and physical conditions that determine coral survival, especially in light of recent and projected future climate impacts that threaten these fragile organisms. Perhaps the most important biophysical driver of coral health is the fine-scale turbulent boundary layer, which acts as a "gatekeeper" to the coral surface through its hydrodynamic control of momentum, mass, and thermal transport processes.

The main objective of this dissertation is to investigate three key modulators of coral turbulent boundary layer dynamics: 1) wave-driven oscillatory flow, 2) topographic surface roughness, and 3) algalcanopy cover. To address these questions, an interdisciplinary research approach was undertaken combining in situ field measurements over algae-covered corals in a fringing reef in Bocas del Toro, Panama, laboratory experiments of rib roughness on a wall-mounted hemisphere using a recirculating water tunnel, and numerical modeling of hydrodynamics and heat transfer from hemispheres using Large Eddy Simulations (LES).

Acoustic Doppler velocimetry (ADV) and particle image velocimetry (PIV) were used to obtain measurements of combined wave-current flow over healthy and algae-covered Siderastrea siderea corals in the reef in Panama. The results demonstrate that the turbulence characteristics of the boundary layer shift in the presence of an algal canopy, from a traditional wall-bounded shear layer to a plane mixing layer. As a consequence of this transition, the algal canopy increases turbulent kinetic energy within the roughness sublayer by $\sim 2.5 \mathrm{x}$ compared to the healthy coral, while simultaneously reducing bed shear stress by nearly an order of magnitude. Lower surface stresses suggest a corresponding reduction in mass transfer at the coral-water interface, leading to negative impacts on a coral's ability to obtain resources and limiting its metabolic productivity.

The impact of coral surface roughness was investigated by collecting PIV measurements and dye visualizations in a recirculating water tunnel for unidirectional flow past a range of 3D-printed coral models 
with rib-type roughness and a model of an actual coral skeleton obtained by computed tomography scanning. The hydrodynamics of the boundary layer and wake structure are shown to depend on Reynolds number and the roughness element spacing, such that densely packed 'd-type' roughness generates flow properties - in particular, mean velocities, streamlines, eddy shedding frequency, and shear stresses - that are similar to a smooth hemisphere model. Widely spaced 'k-type' roughness, in contrast, displays strong vortex ejection from leading edge roughness cavities, resulting in enhanced shear stress (between 50-100\%) and turbulent mixing $(\sim 50 \%)$ in the wake flow.

Lastly, LES models of flow dynamics and heat transfer - used as an analogue for mass transfer - were implemented for unidirectional and oscillatory flows past the same coral geometries used in the laboratory experiments. The numerical results show that under unidirectional forcing, drag forces dominate the physical mechanisms that drive turbulence characteristics and heat transfer; while under oscillatory forcing, inertial forces play a larger role. In general, increased surface roughness and oscillatory flow conditions each enhance heat transfer between 1.2-2.1x. However, in specific flow and roughness combinations- e.g., oscillatory, high Reynolds number flow - the increased surface area resulting from 'd-type' roughness outperforms the greater turbulence produced by 'k-type' roughness with respect to heat transfer enhancement. The complex interaction of flow speed, steadiness of flow, and nature of surface roughness determines the physical mechanisms that optimize trade-offs from surface morphology and creates profound consequences for the bio-physical processes responsible for coral resilience and sustainability. 


\title{
Nomenclature
}

\author{
Roman Symbols \\ $A \quad$ Particle excursion length \\ A Area \\ c Conserved scalar \\ $c_{D} \quad$ Drag coefficient \\ $c_{p}$ Heat capacity \\ $C$ Courant-Friedrichs-Lewy (CFL) number \\ $d \quad$ Water depth \\ d Algal stem diameter \\ $D$ Molecular diffusivity \\ $e_{i j} \quad$ Fluctuating strain rate tensor \\ $f$ frequency \\ $F \quad$ External forcing \\ $F_{D} \quad$ Form drag \\ $h_{c} \quad$ Canopy height \\ $h$ Heat transfer coefficient \\ $H$ Wave height \\ $J$ Mass transfer rate \\ $L \quad$ Wave length \\ $k$ Thermal conductivity \\ $k$ Roughness height \\ $k$ Wavenumber \\ $k_{b} \quad$ Bottom roughness \\ $k_{b c} \quad$ Apparent bottom roughness \\ $K C \quad$ Keulegan-Carpenter number \\ $\ell_{m} \quad$ Turbulent mixing length \\ $\ell_{S} \quad$ Canopy shear length \\ $n$ Number of algae stems \\ $\mathrm{Nu}$ Nusselt number \\ $p$ Pressure \\ $P_{d} \quad$ Pressure drag \\ $\operatorname{Pr}$ Prandtl number, $\nu / \alpha$ \\ $\mathrm{Pr}_{t} \quad$ Turbulent Prandtl number, $\epsilon_{M} / \epsilon_{H}$ \\ $q$ Heat flux \\ $q^{2} \quad$ Turbulent kinetic energy \\ $r_{u w} \quad$ Reynolds stress correlation coefficient \\ Re Reynolds number \\ $R e_{o} \quad$ Wave Reynolds number \\ $R u$ Rugosity
}




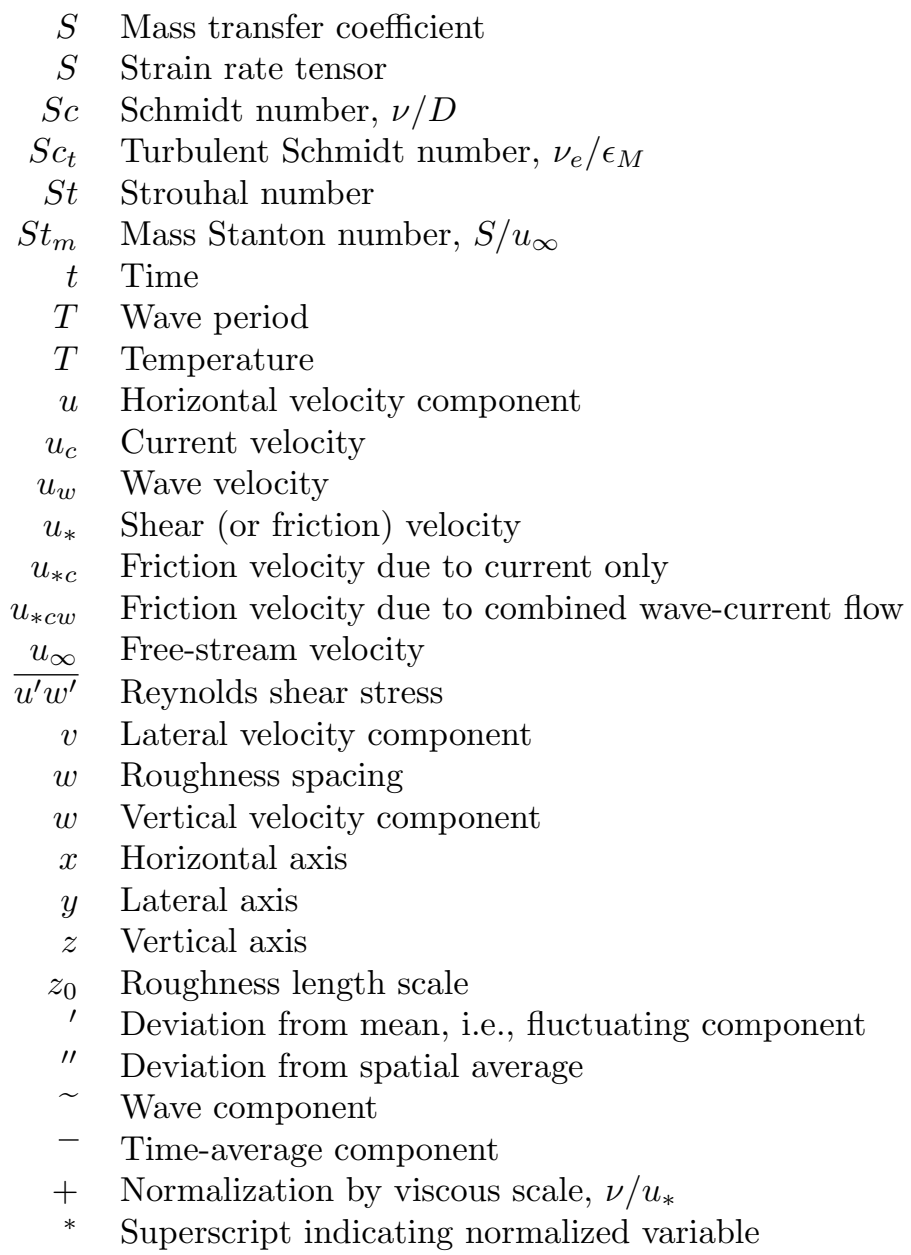

Greek Symbols

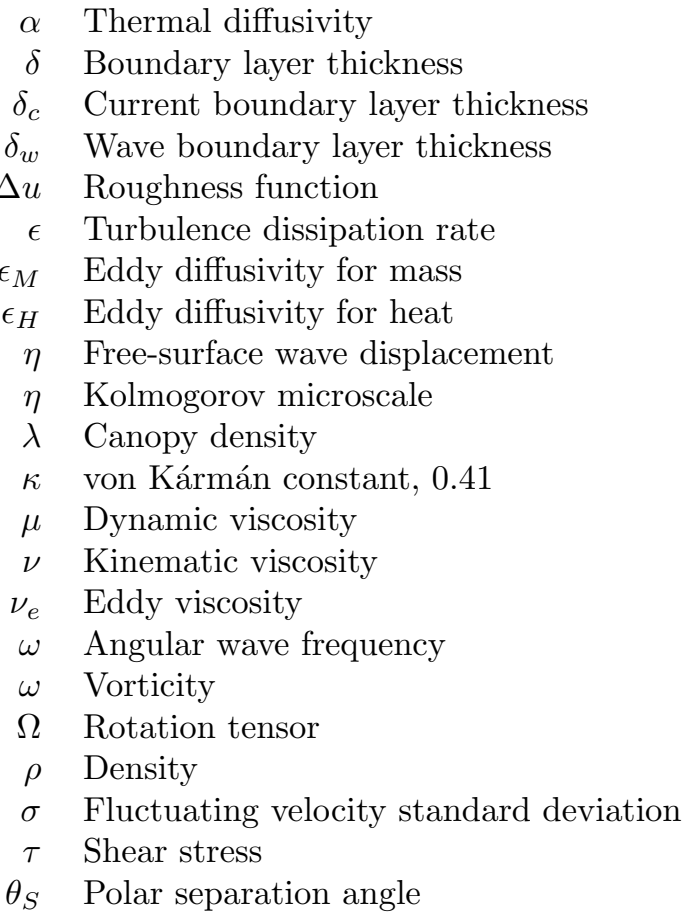




\section{List of Figures}

1.1 Photographs of massive Scleractinian corals from the family Siderastreidae (left) and Faviidae (right), which exhibit two distinct surface morphologies. Images taken in Bocas del Toro,

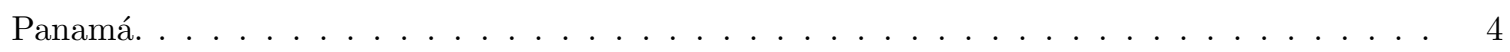

2.1 Colonial assemblage of the branching coral Porites furcata forming the foundation of the reef in Bocas del Toro, Panamá. Other reef species visible include the feather duster Sabellastarte spectabilis, the fire "coral" Millepora alcicornis in the foreground, and various reef fishes in the background. . . . . . . . . . . . . . . . . . . . . 10

2.2 Close-up photographs of the relatively smooth surface of a Siderastrea siderea coral (left) and the highly rugose surface of a Colpophyllia natans coral $($ right $) . \ldots \ldots \ldots \ldots$. . . . . . 22

$2.3 \quad$ Idealized d-type $($ left $)$ and k-type $($ right $)$ bar roughnesses. $\ldots \ldots \ldots \ldots \ldots$

3.1 Site map of island archipelago of Bocas del Toro, Panamá. The study site is located on landward side of Isla Colón. . . . . . . . . . . . . . . . . . . . . . . . . . . . 30

$3.2 \quad$ Schematic of instrument frame with particle image velocimetry experimental setup over healthy Siderastrea siderea coral. The frame was aligned with dominant flow direction to avoid nonlocal turbulence from frame legs. Acoustic doppler velocimeter (ADV) measurements were obtained using the same frame setup with the ADV instrument replacing the laser. . . . . . . 31

3.3 Typical record of instantaneous horizontal velocity $u$ for a 10-min burst. Black dashed line shows background mean, i.e., tidal current velocity, over entire record. Red dotted line plots 60-s running mean and shows consistent stationarity about the burst mean, confirmed quantitatively using reverse arrangements test. Inset plot expands a 30-s segment of the burst to illustrate the spectral wave signal with dominant period of $2.7 \mathrm{~s}$. Data taken from a single ADV measurement bin $\sim 1.5 \mathrm{~cm}$ above healthy coral surface. $\ldots \ldots \ldots \ldots \ldots$ 
3.4 (a) Vectrino acoustic Doppler velocimeter probe tip positioned above a healthy Siderastrea siderea coral. (b) Probe tip positioned above algae-covered S. siderea coral. Note white patch of bleached coral exposed after algae removal for in-canopy flow measurements. . . . . . . . . 33

3.5 Representative power spectral density (PSD) plot of turbulent vertical fluctuations $w^{\prime}$ obtained by ADV for a single measurement bin $\sim 1.5 \mathrm{~cm}$ above the healthy coral. The wave peak $\widetilde{S}_{w w}$, highlighted in red and bound by a $-5 / 3$ fit to the inertial subrange, is subtracted from the total power density (minus noise) to give the turbulent power density $S_{w w}^{\prime}$. . . . . . . . . 35

$3.6 \quad$ (a) Vertical profiles of root-mean-square (rms) horizontal velocity normalized by free-stream rms velocity. Markers indicate heights of measurement bins for ADV, and shaded regions represent \pm one standard deviation from mean. Horizontal blue dashed line indicates approximate height of canopy for algae-covered coral, and gray area represents region of "acoustic reflection" bias, in which data points are less reliable. (b) Vertical profiles of turbulent Reynolds stress normalized by square of the rms free-stream velocity. . . . . . . . . . . . . . . 37

3.7 Comparison of a theoretical combined wave-current logarithmic profile for mean velocity for an inertial boundary layer (in blue) versus measured mean velocity profile over healthy coral (in red). The arrow indicates the transition from wave to current boundary layer, i.e., where the slope of the logarithmic line increases. $\ldots \ldots \ldots \ldots \ldots$

3.8 Vertical profiles of (a) turbulent kinetic energy (b) turbulent production, and (c) turbulent dissipation rate, normalized by square and cube of the root-mean-square free-stream velocity, respectively. Markers indicate heights of measurement bins for ADV, and shaded regions represent \pm one standard deviation from mean. Horizontal blue dashed line indicates approximate height of canopy for algae-covered coral, and gray area represents region of "acoustic reflection" bias, in which data points are less reliable. . . . . . . . . . . . . . . . . . 40

3.9 Comparison of normalized bed shear stress for healthy and algae-covered corals using two independent techniques and showing statistically significant difference in means between healthy and algae-covered corals (Welch's $t$ test, $t=4.1, d f=7, p<0.01$ ). Error bars represent \pm one standard deviation from mean . . . . . . . . . . . . . . . . . . . . . . 41

3.10 (a) Vector field of normalized root-mean-square (rms) velocities over a healthy coral from particle image velocimetry. For clarity, only every third vector is plotted. (b) Plot of turbulent Reynolds stress $\overline{u^{\prime} w^{\prime}}$ normalized by square of rms free-stream velocity. . . . . . . . . . . . . 42

3.11 (a) Plot of turbulent kinetic energy over healthy coral normalized by square of root-meansquare (rms) freestream velocity. Plots of turbulence production (b) and dissipation (c) nor-

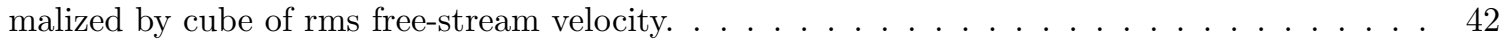


4.1 CAD models of idealized coral geometries. (a) Smooth hemisphere. (b) D-type roughness with $w / k$ ratio equal to 0.5. (c) K-type roughness with $w / k$ ratio equal to $2.0 . \quad \ldots \ldots \ldots \ldots$

4.2 Calcium carbonate skeleton of Platygyra coral (left) and resulting x-ray computed tomography scan (right). The computer model of the skeleton has been scaled up to an approximate diameter of $13.5 \mathrm{~cm}$ to match the size of the idealized models. . . . . . . . . . . . . . . . 51

4.3 Side-angle photograph of the 3D-printed and spray painted coral models (top). Top-down photograph of the coral models (bottom). From left: Smooth hemisphere; hemisphere with $w / k=0.5$; hemisphere with $w / k=2.0$; and CT-scanned coral skeleton. $\ldots \ldots \ldots \ldots$. . . 52

4.4 Photograph of Rolling Hills 1520 recirculating water tunnel with 3D-printed coral model affixed to a glass mounting plate and experimental particle image velocimetry setup. . . . . . 53

4.5 Individual still frame extracted from PIV video clip illustrating particle seeding, density, and focal clarity on the trailing edge of the smooth model. The free-stream horizontal velocity for this image is $15 \mathrm{~cm} \mathrm{~s}^{-1}$, which was most vulnerable to particle streaking due to camera

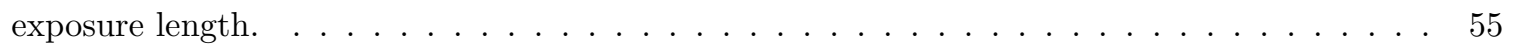

$4.6 \quad$ Photograph of setup for flow visualization using pressurized dye-releasing system. . . . . . . 56

4.7 Left column. Dye visualization of flow past a smooth hemisphere at $5 \mathrm{~cm} \mathrm{~s}^{-1}$, i.e., $R e=6,750$. Each descending row is a still frame from the video record separated by $0.5 \mathrm{~s}$, or 12 frames. Middle column. Flow past smooth hemisphere at $15 \mathrm{~cm} \mathrm{~s}^{-1}$, i.e., $R e=20,250$. Each row is separated by $0.17 \mathrm{~s}$, or 4 frames. Right column. Flow past hemisphere with k-type roughness at $15 \mathrm{~cm} \mathrm{~s}^{-1}$, i.e., $R e=20,250$. Row spacing is $0.17 \mathrm{~s}$, or 4 frames. . . . . . . . . . . . 58

4.8 Distribution of mean horizontal velocity $\bar{u}$. Left column. Low-flow condition: $u_{\infty}=5 \mathrm{~cm} \mathrm{~s}^{-1}$, $R e=6,750$. Right column. High-flow condition: $u_{\infty}=15 \mathrm{cms}^{-1}, R e=20,250$. Row 1. Smooth hemisphere. Row 2. Hemisphere with d-type roughness, $w / k=0.5$. Row 3. Hemisphere with

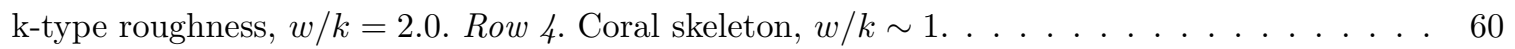

4.9 Polar separation angle for each model. $\ldots \ldots \ldots \ldots \ldots$

4.10 Streamlines of mean horizontal velocity for $R e=6,750$ (left column) and $R e=20,250$ (right

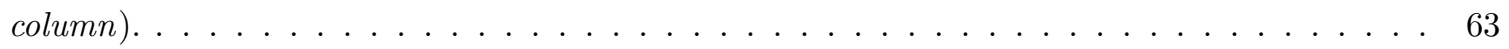

4.11 Vorticity plots for low Re regime (left column) and high Re regime (right column). Row 1 is smooth model, row 2 is d-type roughness model, row 3 is k-type roughness model, and row 4 is coral skeleton model. . . . . . . . . . . . . . . . . . . . . . . . . 65

4.12 Normalized Reynolds shear stress plots for smooth hemisphere at $15 \mathrm{~cm}$ with coordinate rotation into dominant flow direction for all panels $(l e f t)$ and without coordinate rotation for all

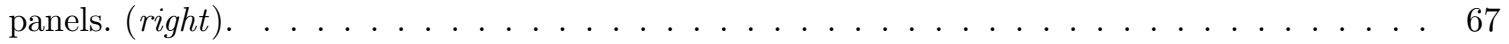


4.13 Normalized Reynolds shear stress plots for $R e=6,750$ (left column) and for $R e=20,250$ (right column). Row 1 is smooth model, row 2 is d-type roughness model, row 3 is k-type roughness model, and row 4 is coral skeleton model. . . . . . . . . . . . . . . . . . . . . . . . 68

4.14 Normalized turbulence production for low-flow (left column) and high-flow (right column) regimes. Row 1 is smooth model, row 2 is d-type roughness model, row 3 is k-type roughness model, and row 4 is coral skeleton model. . . . . . . . . . . . . . . . . . . . . . . . . . . . . 70

4.15 Normalized turbulence dissipation for low flow (left) and high flow (right) regimes. . . . . . . 72

4.16 Joint probability distribution of instantaneous horizontal and vertical velocity fluctuations for low Re regime (top row) and high $R e$ regime (bottom row). . . . . . . . . . . . . . . . . . . . 73

4.17 Single-sided amplitude spectral density (ASD) of instantaneous vertical velocity fluctuations $w^{\prime}$ for $R e=6,750$ (left) and $R e=20,250$ (right). The dashed vertical line indicates a frequency of $0.13 \mathrm{~Hz}$ and $0.40 \mathrm{~Hz}$ for the low and high $R e$ cases, respectively, which each correspond to a Strouhal number of 0.18.

4.18 Dye visualization of flow past k-type hemisphere at $5 \mathrm{~cm} \mathrm{~s}^{-1}$, i.e., $R e=6,750$. Roughness element on the downstream surface disturbs the flow separation and alters the shedding frequency of large-scale vortex structures. The circle of dye at the leading edge indicates a horseshoe vortex (so-called because of its physical similarity as it surrounds the bluff body) and is typical of boundary layer flow interacting with a wall-mounted bluff body. This is also clearly evident in front of the smooth hemisphere in figure 4.6 . . . . . . . . . . . . . . . . . . . . . . . . . 77

4.19 Semi-logarithmic profile plots of horizontal velocity normal to model surface along the leading edge boundary layer for each roughness type at $R e=20,250$. The roughness function $\Delta u$ measures $\sim 1.3 \mathrm{~cm} \mathrm{~s}^{-1}$ for the k-type roughness. . . . . . . . . . . . . . . . . . . . . . . . . 81

4.20 Profiles of turbulent Reynolds shear stress at the leading edge at $x=-4.8 \mathrm{~cm}($ left $)$ and in the downstream wake at $x=9.5 \mathrm{~cm}$ (right) for each roughness type at $R e=20,250$. Peak values for k-type roughness are approximately 50\% and 100\% larger, respectively, compared to smooth and d-type roughness. The much higher vertical location in peak stress for the coral skeleton is a result of the irregular shape of the 3D printed model's base, which elevates the coral skeleton $\sim 3 \mathrm{~cm}$ relative to the other models. . . . . . . . . . . . . . . . . . . . . . . . 82

$5.1 \quad$ Left. Fluid model domain with $w / k=2.0$ hemisphere as example. Right. Model domain showing placement of "high-resolution" box (for unidirectional case) and "super high-resolution" concentric sphere used for targeted grid discretization to improve simulation accuracy in flow regions of greatest interest. . . . . . . . . . . . . . . . . . . . . . . . . . . 93 
$5.2 \quad$ Close-up view in $x-z$ plane of the four model geometries: $(a)$ smooth hemisphere, $(b)$ d-type hemisphere with $w / k=0.5,(c)$ k-type hemisphere with $w / k=2.0$, and $(d)$ coral skeleton. . . 94

$5.3 \quad$ View of mid-plane slice at three levels of zoom illustrating the increasing mesh resolution for the flow areas of interest. Left. View of entire fluid domain mesh for unidirectional $w / k=0.5$ case. Middle. Zoomed view of "high-resolution" mesh region surrounding hemisphere model with maximum element size of $0.5 \mathrm{~cm}$. Right. Further zoom of "super high-resolution" concentric sphere mesh with maximum element size of $0.1 \mathrm{~cm} . \ldots \ldots \ldots$. . . . . . . . . 99

5.4 Streamwise locations of vertical profiles (indicated by dashed vertical lines) used for validation of LES model. . . . . . . . . . . . . . . . . . . . . . . . . . . . . . . . . . . . . . . 101

5.5 Profiles of mean horizontal velocity $\bar{u}$. Top. Low-flow condition: $u_{\infty}=5 \mathrm{~cm} \mathrm{~s}^{-1}, R e=6,750$. . 101

5.6 Profiles of mean horizontal velocity $\bar{u}$. Top. High-flow condition: $u_{\infty}=15 \mathrm{~cm} \mathrm{~s}^{-1}, R e=20,250.102$

5.7 Reynolds shear stress. $\ldots \ldots \ldots \ldots$. . . . . . . . . . . . . . . . . . 103

5.8 Time-averaged streamwise velocity fields for low (left) and high (right) Re unidirectional flow test cases. Maximum and minimum colorbar values are scaled proportionally across each Reynolds number regime so that color field plots are non-dimensional. . . . . . . . . . . . . 105

5.9 Time-averaged streamwise velocity $\bar{u}$ fields for low (left) and high (right) Re oscillatory flow test cases. . . . . . . . . . . . . . . . . . . . . . . . . 106

5.10 Horizontal profiles of mean streamwise velocity $\bar{u}$ (normalized by free-stream velocity $u_{\infty}$ ) at $z=1.5 \mathrm{~cm}$ above bottom wall. Top row: Unidirectional flow test case. Bottom row: Oscillatory flow test case. . . . . . . . . . . . . . . . . . . . . . . . . . 107

5.11 Vertical profiles of mean streamwise velocity $\bar{u}$ (normalized by free-stream velocity $u_{\infty}$ ) for $R e=6,750$ (left) and $R e=20,250$ (right) at one-half diameter downstream from trailing edge of model, i.e., $x / D=0.5$, under unidirectional flow. . . . . . . . . . . . . . . 108

5.12 Field plots of statistical Reynolds shear stress $\overline{u^{\prime} w^{\prime}}$ for low (left) and high (right) Re unidirectional flow test cases. Maximum and minimum colorbar values are scaled proportionally across each Reynolds number regime so that color field plots are non-dimensional. . . . . . . 109

5.13 Field plots of statistical Reynolds shear stress $\overline{u^{\prime} w^{\prime}}$ for low (left) and high (right) Re oscillatory flow test cases. . . . . . . . . . . . . . . . . . . . . . . . . . 110

5.14 Horizontal profiles of mean Reynolds shear stress $\overline{u^{\prime} w^{\prime}}$ (normalized by the square of the freestream velocity $\left.u_{\infty}^{2}\right)$ at an elevation equal to half the model height, i.e., $z / r=0.5$. Top row: Unidirectional flow test case. Bottom row: Oscillatory flow test case. . . . . . . . . . . . . . 111 
5.15 Near-surface close-up field plots of mean velocity magnitude $\overline{\mathbf{u}}$ for unidirectional test cases (top) and oscillatory test cases (bottom) for low and high Re regimes. Maximum and minimum colorbar values are scaled proportionally across each Reynolds number regime so that color field plots are non-dimensional. . . . . . . . . . . . . . . . . . . . . . . . . . . 113

5.16 Surface normal profiles of mean streamwise velocity $\bar{u}$ (normalized by free-stream velocity $\left.u_{\infty}\right)$ for unidirectional flow $(t o p)$ and oscillatory flow (bottom). Profiles are taken at the model leading edge at a location $45^{\circ}$ from the front base and extend outward from the bottom of the roughness cavity. . . . . . . . . . . . . . . . . . . . . . . . . . . . . . 114

5.17 Surface plots of mean wall shear stress for unidirectional (top) and oscillatory (bottom) flows for low and high $R e$ regimes. . . . . . . . . . . . . . . . . . . . . . . . . . . . . . . . . . . . 115

5.18 Time-averaged temperature fields for low (left) and high (right) Re unidirectional flow test cases. . . . . . . . . . . . . . . . . . . . . . . . . 116

5.19 Time-averaged temperature fields for low (left) and high (right) Re oscillatory flow test cases. 117

5.20 Vertical profiles of mean temperature $\bar{T}$ (normalized by background bulk fluid temperature $\left.T_{\infty}\right)$ for unidirectional (top) and oscillatory (bottom) flows at x-axis location one diameter downstream from trailing edge of model, i.e., $x / D=1$. . . . . . . . . . . . . . . . . . . . . 118

5.21 Horizontal profiles of mean temperature $\bar{T}$ (normalized by background bulk fluid temperature $\left.T_{\infty}\right)$ for unidirectional (top) and oscillatory (bottom) flows. Profiles begin adjacent to model trailing edge and extend to end of computational domain. Profiles are taken at a vertical elevation equal to one-half model height, i.e., $z / r=0.5$. . . . . . . . . . . . . . . . . . . . . 119

5.22 Near-surface close-up field plots of mean temperature $\bar{T}$ for unidirectional (top) and oscillatory (bottom) test cases for low and high Re regimes. . . . . . . . . . . . . . . . . . . . . . . . . . 120

5.23 Surface plots of mean wall heat flux for unidirectional (top) and oscillatory (bottom) flows for low and high $R e$ regimes. . . . . . . . . . . . . . . . . . . . . . . . . . . . . . . . . . 121

5.24 Instantaneous eddy vorticity fields for unidirectional low Reynolds number flow, as identified by the Q-Criterion. . . . . . . . . . . . . . . . . . . . . . . . . . . . . 125

5.25 Time series of streamwise velocity (top), pressure (middle), and Q-Criterion (bottom) across one-half of a wave cycle for high $R e$ oscillatory flow past the k-type roughness model. . . . . 126

5.26 Time series of turbulent eddy distribution, as measured by Q-Criterion, for d-type model during accelerating phase after flow reversal, i.e., after $t=\pi / s$, under high $R e$ conditions. . . 127

5.27 Time series of temperature fields for d-type (top) and k-type (bottom) models across one-half wave cycle of high $R e$ oscillatory flow. . . . . . . . . . . . . . . . . . . . . . . . . . . . . . . . 128 
6.1 Photographs illustrating variation in Scleractinian surface morphology. (a) Siderastrea siderea.

(b) Diploria labyrinthiformis. (c) Colpophyllia natans. (d) genus Platygyra, species-level identification unavailable. . . . . . . . . . . . . . . . . . . . . . . . . . . . . . . 134 


\section{List of Tables}

$3.1 \quad$ Flow conditions for healthy $(\mathrm{H})$ and algae-covered $(\mathrm{A})$ corals $\ldots \ldots \ldots \ldots$

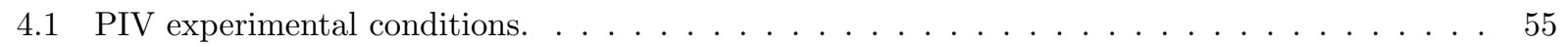

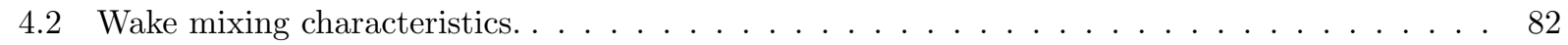

5.1 Description of surface parameters for model geometries. $\ldots \ldots \ldots \ldots$

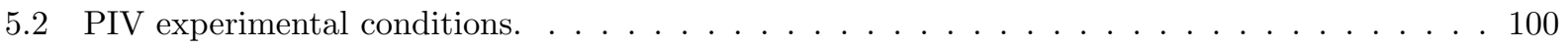

5.3 Turbulence characteristics for each roughness type and flow condition at a point location one model diameter downstream, $x / D=1$, and one model height above the wall, $z / r=1$. . . . 112

5.4 Top: Surface area-averaged and time-mean wall heat flux values $\bar{q}$. Middle: Total amount of heat flux $q_{t o t}$ per unit time. Bottom: Mean heat transfer coefficient $\bar{h}$. . . . . . . . . . . 122 


\section{Acknowledgments}

I have been greatly humbled by and thankful for the support and guidance of numerous individuals throughout the several years of work on this dissertation project. I must first thank my doctoral advisor Prof. Matthew Reidenbach, whose academic and professional counsel has greatly improved the quality of this research and with whom I have had many productive discussions on the fundamental scientific questions that have motivated this project. I would also like to acknowledge the generous and useful feedback provided by the other members of my dissertation committee. In particular, I thank the committee chair, Prof. Haibo Dong, for leading the committee process and for helpful comments on the numerical modeling aspects of this work; Prof. Eric Loth for insightful feedback on the experimental fluid mechanics and for his course on turbulence fundamentals; Prof. Patricia Wiberg for her role as outside committee member, as well as her helpful advice on the fieldwork and ecological importance of the project and whose own research on wave-current boundary layers was critical to the analysis of the field data; and finally, Prof. Silvia Blemker who offered excellent professional development guidance and suggestions for further developing the work for publication, despite the project being outside her area of expertise.

In addition to my doctoral committee, I am indebted to the late Prof. Joseph "Pepe" Humphrey, whose lab was my first academic home upon my arrival at the University of Virginia and who taught me much on the applied physics of fluid mechanics while pursuing my Masters degree. I must thank Prof. Benton Calhoun, who shepherded my MS project to completion following Pepe's passing and who helped bridge the transition to doctoral study with Prof. Reidenbach. Beyond the useful guidance of faculty members, I must also acknowledge the great support and social well-being provided by my fellow lab members. In particular, Craig Eberhardt and Sae-Woong Kil were welcoming and knowledgeable colleagues, who helped make my arrival as a graduate student in Prof. Humphrey's lab a smooth process. Swapnil Pravin joined me in both Pepe's lab group and in the transition to Prof. Reidenbach's lab group and was a good friend with whom I had many interesting conversations both academic and social. Though never a fellow lab member, I thank Christina (Johnson) Pappas, my first MAE and UVA friend, for her enduring friendship and heartening conversations over the years. 
Many fellow graduate students in Prof. Reidenbach's lab created a friendly and welcoming research environment, in particular Jennifer Hansen, Amy Grady, Martin Volaric, and especially Emily Thomas, who was an incredibly kind and friendly office mate, Ross Timmerman, whose field support in Panama was unparalleled, and Elizabeth Murphy, whose research discussions and collegial support have been particularly motivating during the final stretch of the dissertation. I am also indebted to several undergraduate students for assistance with fieldwork in Panama: Daniel Ellis, Alejandro Garcia-Chinchilla, Emma Hollowell, and Mariam Trejos. Special thanks are necessary to J.P. Rippe, who not only contributed tremendously to the fieldwork, but also co-authored the article based on Chapter 3 of this dissertation. I would also like to thank the several undergraduate students who allowed me to help mentor them through their Distinguished Major Projects, in particular Kyle Leathers, Laura Szczyrba, and J.P. Rippe.

I also received continued support from the University of Virginia Department of Mechanical and Aerospace Engineering, as well as the Department of Environmental Sciences. The Panama fieldwork could not have been completed without the assistance of the scientific and support staff at the Smithsonian Tropical Research Institute in Bocas del Toro, Panama. I would also like to extend a special debt of gratitude to the University of Virginia Department of English, which allowed me to periodically sit-in on courses on poetry and literary theory and criticism, particularly the incredibly enlightening courses of Prof. Stephen Cushman and Prof. Jahan Ramazani. The experience allowed me to fulfill my broader intellectual curiosities during my graduate career and was the source of many of my closest friends in the UVA and Charlottesville community.

I would like to thank the many dear friends who made my graduate school experience vastly more worthwhile, especially Adriana Streifer, Campbell Buie, and Rebekah Slodounik. Also, Daniel Fried, Mariusz Kolczykiewicz, and Joey Goodell were motivating work-out friends and always full of interesting conversations. My running group Colin Kielty, Jordan Marshall, and 'coach' William Sbach logged countless hours and miles together, which served as a welcome social outlet and rounded us into Boston Marathon shape. I am grateful for the continued presence in my life of my oldest friends from home, Zach Hines, Tim Szetela, and Evan Greenspoon, who entertain and motivate me more than anyone possibly could. Finally, I could not have accomplished all that I have without the enduring patience, support, and love of my family, especially my siblings Ryan and Nicole and their families, and most especially my parents, who instilled in me a curiosity for life and a love of learning that provides the foundation for everything I do. And last, I am extremely grateful for Kerstin Steitz, who offers me the greatest love, support, and companionship. 


\section{Chapter 1}

\section{Introduction}

"...the vital energies of the corals conquer the mechanical power of the waves; and the large fragments of reef torn up by every storm, are replaced by the slow but steady growth of the innumerable polypifers..."

—Charles Darwin, The Structure and Distribution of Coral Reefs

\subsection{Motivation}

\subsubsection{Economic, Environmental, and Cultural Value of Corals}

Coral reefs have long held a position of tremendous value to the marine ecosystem and global community and of great interest to scientists and naturalists, even before Charles Darwins first systematic and scientific characterization of corals in his 1842 monograph [1. Beyond their spectacular natural beauty, coral reefs generate very high levels of ecosystem productivity and species diversity - more than two orders of magnitude higher than the surrounding open waters - leading to frequent comparisons to tropical rainforests 2]. Despite covering only an estimated $0.1-0.5 \%$ of the ocean floor, coral reefs provide habitat for nearly one-third of all marine fish species and can be found in the coastal waters of over 100 countries 3 [5]. Within this global distribution, hotspots with the greatest coral diversity include southeast Asia and Australia, but corals can also be found in distant locations like the southern tip of Africa and the Mediterranean [6].

Decades of recent work have described and quantified the vast range of ecological goods derived from the direct use of coral reefs and the ecosystem services they provide. Goods sourced from coral reefs include both renewable resources - such as seafood products and raw materials for medicinal purposes - and 
non-renewable resources - such as coral blocks and rubble for construction and raw carbonate sources for production of lime and cement 7, 8. Ecosystem services provided by corals are even more diverse and have been categorized into five major groups: 1) physical structure services, e.g., shoreline protection via wave and current dissipation (as noted by Darwin) and the promotion of mangroves and seagrass beds; 2) biotic services, such as maintenance of habitat and biodiversity, as well as export of organic production to pelagic food webs; 3) biogeochemical services, e.g., nitrogen fixation, control of carbon dioxide and calcium budgets; 4) information services, e.g., climate and pollution records in carbonate skeletons; and 5) social/cultural services, e.g., recreation, aesthetic value, and cultural and religious significance [9]. Roughly estimated, the global economic value of these goods and services is in excess of $\$ 375$ billion annually [10]. In short, corals are an integral part of the global economy, environment, and cultural heritage, and as such, understanding the biophysical mechanisms that control and promote coral survival and success is vital to the preservation of these fragile organisms.

\subsubsection{Mass Transfer and the Role of the Turbulent Boundary Layer}

Coral ecology is governed by a wide range of biological and physical factors, including, but not limited to, such parameters as ocean temperature and acidification 11, 12], the availability of required nutrients and macromolecules [13, 14, the presence of photosynthetic endosymbiotic algae known as zooxanthellae 15 . 16], competition with other reef organisms for light, space, and nutrients [17, 18, direct loss of coral tissue from predation and disease [19, 20], and anthropogenic pressures 21]. Among the complex web of biophysical drivers affecting corals, perhaps the most important consideration is the hydrodynamic interface between the overlying ocean water and the coral surface - a process that can directly and indirectly affect nearly every other biophysical factor. As sessile, benthic organisms, corals must rely on fluid motion for the transport of materials to and from their surfaces. Essential nutrients and dissolved minerals- - uch as $\mathrm{CO}_{2}, \mathrm{PO}_{4}, \mathrm{NH}_{4}$, aragonite, and plankton — need to be transported to the coral for uptake, and waste products and spawning cells - such as $\mathrm{O}_{2}$, sediments, eggs, sperm, and larvae - need to be transported away from the coral for dispersal. Collectively, these dissolved and particulate materials can be treated as scalar masses that require transport by fluid motion and can be investigated across a variety of flow scales.

Coral reef systems and their interface with the overlying water column comprise an enormous range of length and time scales, owing to the reefs widely variable characteristic roughness size and the measurement domain of interest. At the largest reef-level scale on the order of kilometers, tidal and wave forcings connect the open ocean with whole-reef circulation patterns, which provide a direct link for the flux of mass to and from the open ocean [22], and also allow for the dissipation of shore-bound wave energy by reef- 
induced bottom friction and wave breaking 23. At the smallest polyp-level scale on the order of microns, concentration gradients of dissolved materials drive diffusive transport 24 and coral ciliary beating can actively generate vortical flows to enhance mass flux 25]. Between these two extremes of coral length scales is a broad intermediate range on the order of centimeters to meters, which links the two limiting-case regimes.

This intermediate length scale generates and controls both the hydrodynamics and mass transport rates of the fine-scale turbulent boundary layer at an individual coral's interface with the water column, and it is the regime of interest in this dissertation. Under typical forcing conditions of wind-driven spectral waves and tidal currents, the turbulent boundary layer is composed of a superposition of a time-dependent, thin, oscillatory wave boundary layer and a steady (for reasonable time scales) tidal current boundary layer. Known as the "gatekeeper" for thermal, mass, and momentum transport fluxes, the coral turbulent boundary layer has been shown to be strongly modified by the interaction of wave-driven oscillatory flow with the large roughness of the reef topography - a process that drives highly efficient mixing and nutrient uptake rates by the reef [26]. Such efficiency of mixing has been posited as a possible explanation for the so-called "Darwin's Paradox", which questions how reefs can generate unexpectedly large levels of productivity in spite of their highly oligotrophic habitat 27]. However, specific characterization of how coral-scale surface variation (as opposed to reef-scale roughness) affects individual transport rates has not been well studied.

A full description of the hydrodynamics of the intermediate-scale regime requires estimates of both mean quantities - such as tidal current velocity $\bar{u}_{c}$, wave orbital velocity $\tilde{u}_{w}$, wave height $H$, and wave period $T$-and also turbulent statistics - such as Reynolds shear stress $\overline{u^{\prime} w^{\prime}}$, turbulent kinetic energy budget terms, eddy viscosity $\nu_{e}$, and turbulent mixing length $\ell_{m}$. Characterization of energy transfer (i.e., momentum) from the mean flow into statistically defined turbulent motions is important for field, laboratory, and numerical studies, which seek to understand the processes that drive mixing and mass transport and their biophysical control on coral health. Owing to the importance and far-reaching impact of the coral turbulent boundary layer, the primary goal of this dissertation is to investigate the hydrodynamics of turbulent boundary layer flow over corals and to describe how alterations to the coral surface change these dynamics and the consequent implications for mass transfer and coral health. The dissertation describes a series of field measurements over healthy and algal-covered corals in Panama, laboratory water tunnel experiments using 3-D printed coral models of varying surface roughness, and computational fluid dynamics (CFD) simulations of flow structure and mass transfer rates (using heat transfer as a proxy measure) under unidirectional and oscillatory flows. 

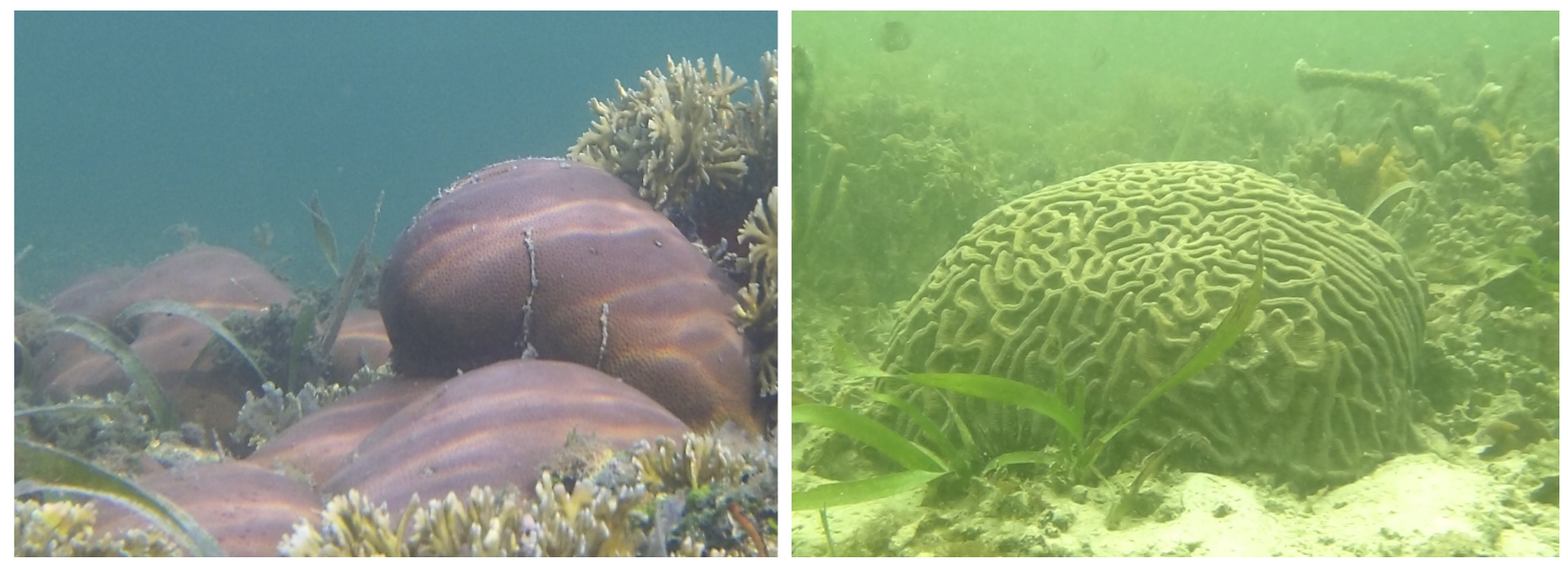

Figure 1.1: Photographs of massive Scleractinian corals from the family Siderastreidae (left) and Faviidae (right), which exhibit two distinct surface morphologies. Images taken in Bocas del Toro, Panamá.

\section{$1.2 \quad$ Research Questions}

Corals of the order Scleractinia, i.e., stony corals that generate a hard calcium carbonate skeleton 11 are known as foundational reef-builders, whose rigid skeletons create and maintain the fundamental structure of reef ecosystems. The importance of Scleractinian corals to reef ecology and the integral role of the fine-scale (order of centimeter) wave-current boundary layer motivate the questions investigated in this dissertation. Numerous studies have demonstrated the influence of the turbulent boundary layer on key ecological functions like nutrient uptake [28], larval dispersal [29], calcification [30], photosynthesis 31], and temperature and acidity modulation 32]. However, relatively few investigations have described the structure and dynamics of the turbulence regimes that physically mediate these mass flux process. Such fine-scale measurements in the field have not been technologically feasible until the recent advent of new micro-profiling measurement devices. Among the turbulence studies undertaken, most have focused predominantly on hydrodynamic descriptions over reef canopies composed of digitate, i.e., finger-like, corals [33, within assemblages of branching corals [34], or over encrusting corals [35]. Missing from the Scleractinian corals cited above, one major and very common group that has been less well studied are massive corals, that is, the families of hemispherical, boulder-shaped corals like Faviidae (commonly called brain corals) and Siderastreidae (commonly called starlet corals) and shown in figure 1.1. The main objectives of this dissertation are to characterize the hydrodynamics of the fine-scale turbulent boundary layer over massive corals, to investigate how modifications to the coral surface affect the turbulent boundary layer, and to numerically simulate how these modifications affect water-column mixing and mass transfer. In this way, the research aims to fill gaps

\footnotetext{
${ }^{1}$ The other major order of corals is Alcyonacea, or soft corals, which lack a calcium carbonate skeleton, and are beyond the scope of this work - though the fleshy morphology of Alcyonacea undoubtedly creates an interesting fluid-structure interaction that deserves study in its own right. Less common coral orders include Antipatharia, or black corals, and Helioporacea, or blue corals.
} 
in our understanding of coral boundary layers, mass flux, and the consequent impacts on individual coral health and entire reef ecosystem sustainability.

The experiments in this study will address three important environmental factors commonly present in a coral's habitat that have the ability to modulate the turbulent boundary layer and affect rates of mass flux: 1) wave-driven oscillatory flow, 2) surface roughness, and 3) overlying algal-canopy cover. Representing a typical coral environment found in a reef habitat - that is, an hemispherical coral with rough surface topography exposed to wave-swept flows and potentially susceptible to algal-canopy cover-these modulating factors complicate the dynamics of the coral-water interface and point to specific limitations in our understanding of coral boundary layers. Guided by these considerations, the overarching research questions that motivate this dissertation as a whole are described below.

1. What are the fine-scale mixing and turbulent hydrodynamics of boundary layer flow over smooth, hemisphere-shaped Scleractinian corals under steady, unidirectional flows?

The conditions considered in the above question, i.e., a smooth surface and unidirectional flow, represent a baseline state against which subsequent experimental conditions will be compared. In this way, the study seeks to build levels of complexity that represent a spectrum of realistic environmental conditions, while attempting to distinguish the effects of each added parameter. Hydrodynamic descriptions of the finescale boundary layer and downstream wake characteristics were achieved by collecting a series of experimental measurements in the Mechanical and Aerospace Engineering Department's recirculating water tunnel at the University of Virginia (UVA). Measurements of mean velocity and turbulent statistics were made over an idealized coral model represented by a smooth plastic hemisphere constructed through fused depositional modeling. Quantitative data records were collected using 2-dimensional particle image velocimetry (PIV), while qualitative descriptions were made using dye-injected flow visualization. Additionally, descriptions of boundary layer hydrodynamics and downstream wakes were numerically modeled through Large Eddy Simulations (LES) using commercially available computational fluid dynamics (CFD) code from ANSYS, Inc. Results from the numerical simulations were validated using the experimental water tunnel measurements, and simulations of surface mass flux were undertaken using a convective heat transfer module as an analogue for mass transfer.

2. How does oscillatory flow-forced by wind-driven spectral waves in situ or simulated as monochromatic waves in numerical simulations - affect the hydrodynamics of the turbulent boundary layer over corals, and what are the implications for mass flux compared to equivalent unidirectional flows? 
The defining characteristic of combined wave-current boundary layer hydrodynamics is that key parameters are time-dependent, i.e., the amplitudes of values such as Reynolds stresses and bed shear vary throughout the wave cycle (and since the signs of these values also vary with wave phase, i.e., flow direction, the most physically relevant time-average is the root-mean-square value). For both rigid and flexible canopies, oscillatory flow drives turbulent stresses deeper into the canopy and increases bed shear stresses compared to equivalent unidirectional flows 33,36 . For flows over smooth and rough flat surfaces, oscillatory flow alters the boundary layer structure and enhances mass transfer up to $60 \%$ over comparable steady flows [37]. However, for massive corals or other spherical bluff bodies, little is known about how the superposition of a time-varying wave boundary layer onto a steady current boundary layer affects turbulence dynamics and mass flux.

To address this gap in the literature, an investigation of combined wave-current flow over massive corals was undertaken during two summer field deployments to a fringing coral reef in Bocas del Toro, Panama. Two-dimensional velocity measurements were collected using a custom-made in situ PIV system, and full 3-dimensional velocity measurements were made using a profiling ADV. Field measurements were only able to characterize combined wave-current flows - rather than pure oscillatory or pure steady flowsbecause conditions were consistently wavy. However, spectral analyses were able to decompose time-averaged derived quantities such as Reynolds stresses and bed shear into either wave or current contributions. In order to resolve a complete volumetric 3-dimensional record of the hydrodynamics, Large Eddy Simulations of pure current and pure wave conditions were modeled, which also allowed for the estimation of mass transfer rates across the coral surface for both flow regimes.

\section{How does a coral's surface roughness affect the hydrodynamics of the turbulent boundary layer} for unidirectional and oscillatory flows, and how does roughness impact rates of mass transfer?

Previous studies of surface roughness have predominately investigated flat surfaces with bar-type roughness elements, composed of well-ordered ratios of roughness element spacing width $w$ to roughness element height $k$. Ratios less than unity, i.e., $w / k<1$, are defined as "d-type" roughness and exhibit stable internal flow within the cavities between elements. By contrast, surface roughness with ratios greater than unity, i.e., $w / k>1$, are classified as "k-type" roughness and characterized by the ejection of vortices into the overlying boundary layer 38 . (More recent classifications rely on the ratio of pressure drag to frictional drag, which will be discussed in the following chapter). A second important method to describe surface topography is the concept of rugosity, which quantifies the fine-scale variation of surface elevation. Rugosity is calculated as the ratio of the total surface area to the projected 2-dimensional planar surface area. For massive corals, the surface topography can vary widely, from nearly smooth corals with low rugosity like Siderastrea siderea 
to deeply ridged brain-type corals with high rugosity, like Colpophyllia natans.

To investigate how surface roughness affects the turbulent boundary layer and wake dynamics, velocity measurements were made over and downstream of three idealized coral geometries possessing a range of $w / k$ ratios and representing both $\mathrm{d}$ - and k-type roughness. Additional velocity measurements were collected over a computed tomography $(\mathrm{CT})$ model of an actual brain coral skeleton. All models were constructed using fused depositional modeling and tested experimentally in the UVA recirculating water tunnel using PIV and dye-injected flow visualization. Experimental data were used to validate LES models of flow over the same geometries for unidirectional flow, and the LES models were extended to simulate oscillatory flow conditions and surface heat (and mass) flux.

\section{How does turf algae canopy on the surface of a massive coral affect the structure and dynamics} of the turbulent boundary layer, and what are the implications for mass transfer?

Assemblages of turf algae can invade and overgrow the surface of corals causing significant degradation to community biodiversity and to physical reef structures 17 . Previous work has shown that presence of an algal canopy can alter the boundary layer structure by increasing the thickness of the mean velocity profile 39]; however, no studies have attempted to measure the turbulence statistics of flow in an algal canopy. A flexible algal canopy is hypothesized to resemble more commonly studied terrestrial canopy flow, characterized by an inflection point in the mean velocity profile, which generates Kelvin-Helmholtz instabilities that propagate to fully developed turbulence. The presence of a canopy is expected to buffer the coral surface from turbulent mixing processes, which will strengthen the mass transfer bottleneck, despite the additional drag produced by algal elements. In order to describe the turbulence structure within a turf algae canopy, measurements were collected using a 3D profiling acoustic Doppler velocimeter over a massive S. siderea coral overgrown by algae during field experiments in Bocas del Toro, Panama.

For each main research question, the ultimate parameter of ecological interest is the mass transfer rate, which largely determines the health and sustainability of corals. Since the hydrodynamics of the turbulent boundary layer play a governing role in setting rates of mass flux (along with molecular diffusivities), this dissertation aims to describe key boundary layer measures - such as mean velocity, Reynolds stresses, TKE budgets - subject to various surface alterations, and consequently, the impact on mass transfer due to these alterations. Beyond specific contributions to coral hydrodynamics and ecology, this research also seeks to extend in a more general manner the scientific community's basic understanding of turbulent boundary layers over rough surfaces, which constitute a vital and active area of research with an enormous range of applications. 


\subsection{Contribution of Results}

\subsubsection{Novelty and Significance}

The research fields of boundary layer flow, convective heat transfer, and coral ecology are each well established and have been deeply investigated throughout scientific history. The novelty of this dissertation lies primarily in the unique intersection of these three research areas and the application of previously developed physics to new, interdisciplinary conditions, such wavy flow in coral habitats. In particular, the results of chapter 3 present the first quantitative description of the turbulence regime for an algal canopy growing on the surface of an individual coral. Chapter 4 provides the first experimental investigation of and hydrodynamic results for flow past a wall-mounted hemisphere with various rib roughness. Finally, chapter 5 extends the results of chapter 4 by implementing the first Large Eddy Simulations of convective heat transfer from rough hemispheres subject to both unidirectional and oscillatory flow conditions. Each chapter presents novel physical descriptions of previously unstudied hydrodynamic systems and offers insight into the potential ecological implications that result from these various boundary layer alterations. In sum, the results derived from the combination of field work, laboratory experiments, and numerical simulations not only add to our knowledge of the various conditions affecting boundary layer flow and its turbulence characteristics, but also permit a better understanding of the biophysical interactions that are critical to the health and resilience of corals and the many significant ecological, economic, and social benefits they provide. 


\section{Chapter 2}

\section{Background Theory and Literature}

\section{Review}

In this chapter, I review the fundamental biology of corals and provide an overview of the theory of key fluid dynamic processes that affect the coral lifecycle. Relevant experiments and field studies from the literature that describe the current state of understanding of coral hydrodynamics will be presented and used to establish gaps in the scientific record that this dissertation seeks to address. Section 2.1 will briefly outline basic coral anatomy and the coral lifecycle, including reproduction, feeding, and colony formation, and discuss the importance of concentration boundary layers to corals. Sections 2.2 and 2.3 present the basic equations of motion governing turbulent flows, boundary layers, and combined wave-current flows. Sections 2.4 and 2.5 describe the hydrodynamics resulting from alterations to a typical coral surface in the form of algal canopy cover and high surface roughness. Chapter 2 closes with a review of scalar mass transport and the advection-diffusion equations governing this important physical control on coral health.

\subsection{Coral Ecology}

\subsubsection{Fundamentals of the Coral Lifecycle}

Corals are marine invertebrate animals classified within Linnaean taxonomy as part of the phylum Cnidaria and class Anthozoa [6] The focus of this dissertation is the stony coral of the order Scleractinia, described perviously in Chapter 1 as a foundational "reef builder" that generates a hard calcium carbonate skeleton. An individual coral is composed of a colonial assemblage of many individual polyps, which are the constituent body morphologies responsible for the uptake of dissolved crystalline $\mathrm{CaCO}_{3}$, called aragonite, 


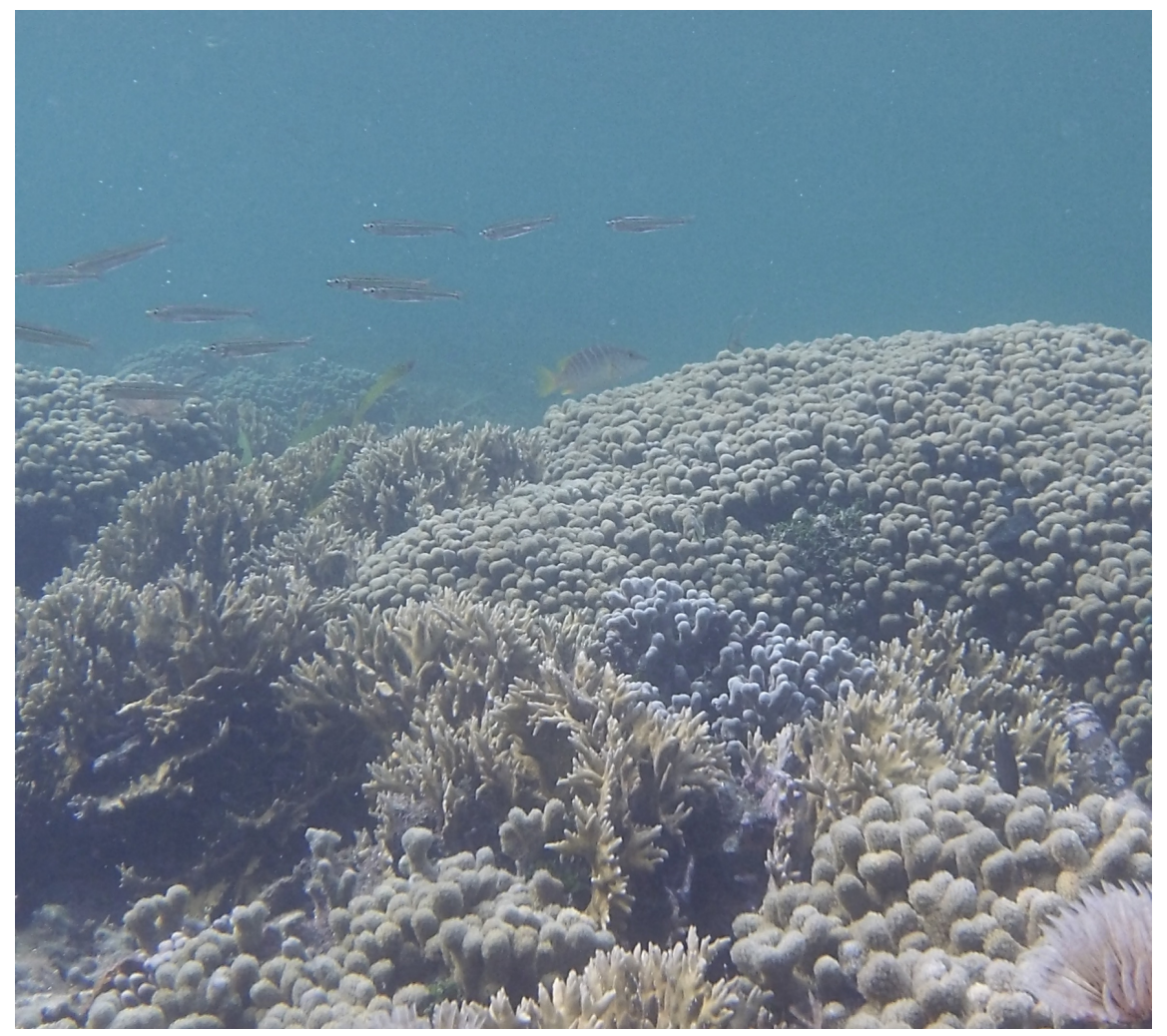

Figure 2.1: Colonial assemblage of the branching coral Porites furcata forming the foundation of the reef in Bocas del Toro, Panamá. Other reef species visible include the feather duster Sabellastarte spectabilis, the fire "coral" Millepora alcicornis in the foreground, and various reef fishes in the background.

from the ocean water column. Each polyp subsequently deposits the calcium carbonate through its basal plate to create a permanent skeleton structure that forms the basis of the reef community. Like most coral species, the Scleractinian corals in this study asexually bud from an initial "parent" polyp to produce "offspring" polyps, which continue the cycle until a familiar colony formation is created, as shown in Figure 2.1. Upon establishment of the adult colony, the vast majority of corals undertake hermaphroditic sexual reproduction, in which a synchronized release of sperm and eggs occurs during an annual spawning event [40]. Timed to allow for predominantly cross-fertilization in the water column, coral broadcast spawning generates freeswimming larvae, which actively respond to chemical cues in the water column directing them to suitable locations for attachment and growth 41 .

As sessile animals, corals have evolved two distinct strategies to obtain nutrition without the aid of locomotion. First, nearly all reef-building corals residing in the photic zone, i.e., at a water depth at which light still penetrates, contain millions of endosymbiotic unicellular dinoflagellates called zooxanthellae within individual polyps. Zooxanthellae are autotrophic organisms that undertake photosynthesis, providing coral cells with oxygen and complex organic compounds needed for respiration and metabolic processes while simultaneously removing the polyp's $\mathrm{CO}_{2}$ and nitrogen waste products. Additionally, zooxanthellae are re- 
sponsible for creating the brilliant diversity of coloration in corals, and the expulsion of zooxanthellae during conditions of environmental stress result in the familiar bleaching patterns seen on degraded corals. The second strategy corals have adopted for nutrient intake is direct predation of zooplankton and suspended organic particles. As nocturnal predators, individual coral polyps are able to extend their tentacles to trap microscopic plankton, which are then dissolved within the polyp gut. Corals are also able to predate by creating a surface mucus layer, which ensnares free-floating or swimming prey and are subsequently transported to the gut for consumption.

\subsubsection{Benthic Boundary Layer and Concentration Gradients}

The marine benthic boundary layer (BBL) is a specialization of the general boundary layer concept used to describe the region of near-bottom ocean water and its frictional interaction with the benthos, i.e., the community of flora and fauna living in or near the seafloor. The marine BBL, similar to traditional hydrodynamic boundary layers, is distinguished by sharp gradients in velocity, momentum, mass concentrations, and thermal energetics and can be defined across a range length scales. In deep-water regimes, the marine BBL can extend tens of meters in elevation above the seafloor; whereas, in more typical shallow-water reef communities, the BBL thickness is on the order of tens of centimeters to meters 42 . When nutrients or dissolved chemicals are either produced or consumed by the benthos - for example, the uptake of ammonium or phosphate by corals, the release of oxygen and dissolved organic matter by autotrophs, or the consumption of zoo- or phytoplankton by heterotrophs - the formation of a concentration boundary layer occurs, which can influence metabolic rates and limit growth of benthic organisms.

Initial characterizations of the BBL relied on applying fundamental engineering concepts of scalar mass and heat transfer (to be discussed in Section 2.6) to environmental systems. Early evidence of large-scale, $O$ (meter), concentration boundary layer formation came from experimental observations of benthic filtering by suspension-feeding bivalves, which were seen to control annual spring phytoplankton blooms in San Francisco Bay [43, 44. Similarly, in the St. Lawrence River estuary, increased rates of bivalve metabolism were found to correlate with higher current speeds, suggesting an enhancement in turbulent mixing that replaced nutrient-depleted benthic water with nutrient-rich surface water 45 . Laboratory experiments subsequently quantified these field observations, demonstrating that the ratio of pumping velocity $u_{j}$ to boundary shear velocity $u_{*}$ determines the phytoplankton removal efficiency — the parameter responsible for establishing the water column concentration gradient 46 . Within the BBL exist several other smaller scale boundary layersdepending on the scale of investigation - such as the momentum boundary layer (MBL), the viscous boundary layer (VBL), and the diffusive boundary layer (DBL). Each boundary layer can possess a concentration 
gradient of its own so that larger boundary layers represent the integrated effects of smaller scale layers [42].

At the smallest scales, $O$ (¡millimeter), concentration gradients and vertical mass transport are controlled by molecular diffusion $D$ at the coral surface. In this region, surface friction retards the flow so that viscosity becomes the dominant control on fluid motion, and rates of molecular diffusion become larger than rates of turbulent eddy diffusion, thereby creating a concentration gradient called the diffusive boundary layer. The size of the DBL is determined by surface roughness, flow speed, and the molecular diffusivities of relevant dissolved materials (e.g. $\mathrm{O}_{2}, \mathrm{CO}_{2}, \mathrm{PO}_{4}^{3-}, \mathrm{NH}_{4}^{+}$) 47. As the DBL is the final barrier for nutrient uptake by individual coral polyps, it has become known as the mass transfer "bottleneck" [48. Numerous studies have shown that larger turbulence-induced wall shear stress $\tau$ reduces the thickness of the diffusive boundary layer and enhances mass flux at a water-substrate interface 49 51]. Additionally, higher levels of turbulent kinetic energy $k$ in the intermediate-scale boundary layer near the benthic surface thin the DBL, causing it to more smoothly follow the bottom bed topography [52] and increasing mass transport [53]. Finally, oscillatory flow driven by surface waves also disrupts the formation of boundary layer concentration gradients and breaks down the diffusive boundary layer - especially during flow reversal — thereby enhancing mass flux $[54$.

\subsubsection{Turf-forming Macroalgae Degradation}

In reef flat communities, many other species directly compete with corals for resources in the oligotrophic, i.e., nutrient-poor, tropical and sub-tropical coastal waters. In particular, benthic macroalgae compete with corals for space, light, and nutrients [55], often exhibiting a competitive advantage under conditions of limited herbivory or excessive nutrient loading [56. The algae typically found in coral reef systems are multi-specific assemblages of green, red, and brown seaweeds with filamentous Cyanobacteria [57. Individual species identification is generally not performed since the biodiversity of reef turf algae is so high, containing greater than 20 species in $\sim 1 \mathrm{~cm}^{2}$ [58]. Coral-algae interactions result in a number of detrimental effects to coral health including: allelopathic chemical attack [59], epithelial sloughing [60], shading, and overgrowth 17]. An important potential hydrodynamic impact arises when macroalgae begin to colonize the surface of a coral and form a turf algae canopy, which can significantly alter the normal hydrodynamics within the fine-scale boundary layer and impede rates of vertical transport and mixing. The reduction in mass transfer due to a canopy degrades a coral's ability to obtain nutrients and endangers the productivity and diversity of the entire reef. 


\subsection{Turbulent Boundary Layer Theory}

\subsubsection{Equations of Motion}

The instantaneous velocity and pressure fields for an incompressible fluid flow in three dimensions are governed by the Navier-Stokes equations, given in tensor notation as

$$
\begin{gathered}
\frac{\partial u_{i}}{\partial t}+u_{j} \frac{\partial u_{i}}{\partial x_{j}}=-\frac{1}{\rho} \frac{\partial p}{\partial x_{i}}+\nu \frac{\partial^{2} u_{i}}{\partial x_{j} \partial x_{j}}+F_{i}, \\
\frac{\partial u_{i}}{\partial x_{i}}=0
\end{gathered}
$$

where $p$ is pressure, $\nu$ is the kinematic viscosity of the fluid, and $F_{i}$ is any external body force 61. 1. Equation 2.2 is the continuity equation, which ensures fluid incompressibility, i.e., non-divergence of the velocity field. For a turbulent flow, the instantaneous velocity and pressure fields can be decomposed into a temporal mean component and a deviation from the mean component - a procedure called Reynolds decomposition - such that $u_{i}=\bar{u}_{i}+u_{i}^{\prime}$ and $p=\bar{p}+p^{\prime}$, where the overbar indicates a time-averaged quantity and the prime indicates an instantaneous fluctuation. By definition, the average of the fluctuating components over time is zero, i.e., $\overline{u_{i}^{\prime}}=\overline{p^{\prime}}=0$. The temporal Reynolds decomposition requires stationary flow, i.e., $\partial \bar{u}_{i} / \partial t=0$; otherwise, ensemble averaging over many instantiations from the same initial conditions is required.

To derive the mean momentum and continuity equations that describe a turbulent flow field, the decomposed velocity and pressure terms are substituted into the instantaneous Navier-Stokes equations (2.1) 2.2), and these equations are averaged with respect to time. As an example of this procedure, substitution of the Reynolds decomposed velocity into the continuity equation 2.2 and subsequent averaging with respect tot time yields

$$
\overline{\frac{\partial}{\partial x_{i}}\left(\bar{u}_{i}+u_{i}^{\prime}\right)}=\overline{\frac{\partial \bar{u}_{i}}{\partial x_{i}}}+\overline{\frac{\partial u_{i}^{\prime}}{\partial x_{i}}}=\frac{\partial \bar{u}_{i}}{\partial x_{i}}+\frac{\partial \overline{u_{i}^{\prime}}}{\partial x_{i}}=0 .
$$

Recalling that $\overline{u_{i}^{\prime}}=0$, this requires that the final term, i.e., the gradient of mean fluctuations, also equals zero, which yields the continuity equation for the mean flow: $\partial \bar{u}_{i} / \partial x_{i}=0$. This expression can be subtracted from the total continuity equation 2.2 to produce the continuity equation for the turbulent flow, i.e., $\partial u_{i}^{\prime} / \partial x_{i}=0$. In this way, we have demonstrated that the instantaneous, the mean, and the turbulent components of the velocity field are all non-divergent.

\footnotetext{
${ }^{1}$ The derivation of the turbulent equations of motion and boundary layer scaling presented in this section can be found in any introductory text on fluid mechanics, and the organization here primarily follows 61 .
} 
A similar procedure (omitted here for clarity) can be undertaken for each of the terms of the instantaneous momentum equation (2.1), and assuming no external forcing, yields the mean momentum equation

$$
\frac{\partial \bar{u}_{i}}{\partial t}+\bar{u}_{j} \frac{\partial \bar{u}_{i}}{\partial x_{j}}=-\frac{1}{\rho} \frac{\partial \bar{p}}{\partial x_{i}}-\frac{\partial}{\partial x_{j}}\left(\overline{u_{i}^{\prime} u_{j}^{\prime}}\right)+\nu \frac{\partial^{2} \bar{u}_{i}}{\partial x_{j} \partial x_{j}} .
$$

Comparing the mean momentum equation (2.4) to the instantaneous momentum equation (2.1), it is evident that the turbulent fluctuations in the flow field have introduced an additional stress term $\overline{u_{i}^{\prime} u_{j}^{\prime}}$, called the Reynolds stress tensor. Factoring out a common flux divergence operator from the final two terms and multiplying by density, we can then define these terms as the total shear stress $\tau$, which is composed of a turbulent component and a viscous component: $\tau=-\rho \overline{u_{i}^{\prime} u_{j}^{\prime}}+\mu\left(\frac{\partial \bar{u}_{i}}{\partial x_{j}}+\frac{\partial \bar{u}_{j}}{\partial x_{i}}\right)$. Except very near a boundary where fluctuations are small and mean gradients are large, turbulent stresses are significantly larger than viscous stresses. The origins of Reynolds stresses lie in the nonlinear advection term in the Navier-Stokes equations, i.e., $u_{j} \frac{\partial u_{i}}{\partial x_{j}}$, and can be thought of conceptually as the transfer of momentum by turbulent fluctuations from the mean flow to the turbulent flow.

\subsubsection{Turbulent Kinetic Energy Budget}

Having established the governing equations for the velocity and pressure fields for an incompressible turbulent flow, it is now possible to explore the sources and sinks of turbulent kinetic energy (TKE) within the flow. A general procedure for obtaining kinetic energy is to integrate the scalar product of velocity and the change in momentum, i.e., $\mathrm{KE}=\int u_{i} \cdot d\left(p_{i}\right)=\frac{1}{2} m u_{i}^{2}$, where $p_{i}=m u_{i}$ is momentum. Analogously, the turbulent kinetic energy equation is obtained by multiplying the turbulent velocity $u_{i}^{\prime}$ by the turbulent momentum equation $\frac{\partial u_{i}^{\prime}}{\partial t}$ (itself obtain by subtracting the mean momentum equation 2.4 from the total momentum equation (2.1) and averaging the scalar product. Ignoring buoyancy and other external forcings, this yields

$$
\frac{\partial q^{2}}{\partial t}+\bar{u}_{j} \frac{\partial q^{2}}{\partial x_{j}}=\underbrace{-\frac{\partial}{\partial x_{j}}\left(\frac{1}{\rho} \overline{p u_{j}^{\prime}}+\overline{q^{2} u_{j}^{\prime}}-2 \nu \overline{u_{i}^{\prime} e_{i j}}\right)}_{\mathrm{I}}-\underbrace{\overline{u_{i}^{\prime} u_{j}^{\prime}} \frac{\partial \bar{u}_{i}}{\partial x_{j}}}_{\mathrm{II}}-\underbrace{2 \nu \overline{e_{i j} e_{i j}}}_{\mathrm{III}},
$$

where $q^{2} \equiv \frac{1}{2}\left(\overline{u^{\prime 2}}+\overline{v^{\prime 2}}+\overline{w^{\prime 2}}\right)$ is turbulent kinetic energy per unit mass and $e_{i j} \equiv \frac{1}{2}\left(\frac{\partial u_{i}^{\prime}}{\partial x_{j}}+\frac{\partial u_{j}^{\prime}}{\partial x_{i}}\right)$ is the fluctuating strain rate tensor 2 The left-hand side of equation 2.5 represents the temporal rate of change in

\footnotetext{
${ }^{2}$ A note on coordinate systems: Throughout this dissertation, the streamwise direction is denoted by $x$ with corresponding horizontal velocity $u$, the lateral direction is denoted by $y$ with cross-flow velocity $v$, and the transverse direction, i.e., wallnormal, is denoted by $z$ with corresponding vertical velocity $w$.
} 
TKE and the advection of TKE, respectively. Term I on the right-hand side (r.h.s.) describes the quantities responsible for the spatial transport of TKE (a result of the leading divergence operator). The physical mechanisms described by each term are pressure-drive transport, turbulence-driven transport, and viscousdriven transport, respectively. Term II on the r.h.s. is the shear production of turbulent kinetic energy, resulting from the interaction of the Reynolds stress with the mean velocity gradient - in effect, a transfer of kinetic energy from the mean flow to the turbulent flow. Term III on the r.h.s. is the viscous dissipation of turbulent kinetic energy, which is a kind of "smearing out" by fluid viscosity of the smallest scale turbulent fluctuations into thermal energy. Dissipation, also represented as $\epsilon$, can be calculated directly through the velocity gradients of the strain rate tensor or though spectral fitting to a theoretical Kolmogorov spectrum. For fully developed turbulence in equilibrium, shear production and viscous dissipation directly balance each other and transport terms are negligible.

\subsubsection{Boundary Layer Scaling}

Flow over the surface of a coral is a specific example of the general class of wall-bounded shear flows, the major features of which I describe below. In general, turbulent flow bounded by a solid wall requires a "no-slip", i.e., zero velocity, boundary condition at the surface. This boundary condition creates a vertical gradient in velocity that decreases until the free-stream velocity $u_{\infty}$ is reached, resulting in the formation of a turbulent boundary layer (TBL), the thickness of which is typically defined as the height at which the mean horizontal velocity reaches 95 or $99 \%$ of $u_{\infty}$. The TBL can be divided into three regions: 1) the inner layer, in which fluid viscosity $\nu$ governs flow properties, 2) the outer layer, in which flow is inviscid and the TBL thickness $\delta$ controls turbulent eddy size, and 3) the overlap layer, in which neither $\nu$ nor $\delta$ directly influences the flow characteristics and inertia dominates. The three boundary layer regions are analogous to the viscous range, inertial range, and forcing range for homogeneous, isotropic turbulence.

First, the inner layer, also called the viscous sublayer, is the region of the turbulent boundary layer in which turbulent fluctuations are small and a uniform viscous stress dominates. This constant viscous stress is set equal to the wall shear stress $\tau_{0}$, which is used to define a normalizing scale called the friction velocity $u_{*} \equiv$ $\sqrt{\tau_{0} / \rho}$, with dimensional units $[L] \cdot[t]^{-1}$. In the viscous sublayer, velocity $u$ is nondimensionalized by $u_{*}$, so that the dimensionless velocity becomes $u_{+} \equiv u / u_{*}$. Similarly, the wall normal height $z$ is nondimensionalized by the viscous scale $\nu / u_{*}$, so that dimensionless wall units become $z_{+} \equiv z u_{*} / \nu$. The constant viscous stress in the inner layer requires that the velocity gradient also remain constant (noting that since $\overline{u^{\prime} w^{\prime}}=0$ here, then $\tau_{0}=\mu \frac{\partial u}{\partial z}$ ), which implies that the velocity distribution depends linearly on height only. This gives rise 
to the famous law of the wall, which takes the universal functional form

$$
\frac{u}{u_{*}}=f\left(z_{+}\right) .
$$

The linear velocity profile has been shown to be valid until approximately $z_{+} \sim 5$, which determines the thickness of the viscous sublayer.

Next, the outer region of the turbulent boundary layer is characterized by a flow structure resembling inviscid, wall-free turbulence and is governed by the total thickness of the boundary layer $\delta$. Reynolds stresses found in this region impart a drag on the mean velocity resulting in a reduction in velocity magnitude compared to the free-stream velocity, i.e, $u_{\infty}-u(z)$. The size of the defect in velocity is expected to be proportional to the wall friction velocity $u_{*}$ and a function of the distance from the wall normalized by the boundary layer thickness. This effect is called the velocity defect law and is given by the functional form

$$
\frac{u_{\infty}-u}{u_{*}}=f\left(\frac{z}{\delta}\right) .
$$

Finally, there exists a region of overlap between the inner and outer layers in which neither $\nu$ nor $\delta$ directly determines the flow characteristics. Since the inner layer and outer layer possess different scaling terms for height $z$ - the viscous scale $\nu / u_{*}$ and boundary layer scale $\delta$, respectively - in order to define the overlap region, it is necessary to match the law of the wall and the velocity defect law by simultaneously taking the limits $z u_{*} / \nu \rightarrow \infty$ and $z / \delta \rightarrow 0$. Rearranging equations (2.6) and (2.7) in terms of their velocity gradients $\partial u / \partial z$ and integrating yields the velocity distribution for the overlap layer

$$
\frac{u}{u_{*}}=\frac{1}{\kappa} \ln \frac{z u_{*}}{\nu}+C,
$$

where $\kappa$ is the universal von Kármán constant equal to 0.41 and $C$ is an integration constant empirically shown to equal 5.0 for a smooth plate. The overlap region is also known as the logarithmic layer because of the logarithmic distribution of velocity, or alternatively, the inertial layer because little turbulent production or dissipation occurs, merely an inertial cascade from large to small eddies through nonlinear inviscid processes. The $\log$ law distribution has been shown to be valid for $30<z_{+}<300$, which is typically only about $20 \%$ of the total boundary layer thickness — the majority of which is governed by the velocity defect law $3^{3}$

\footnotetext{
${ }^{3}$ The "missing" region from $5<z_{+}<30$ is called the buffer layer, in which the velocity profile is neither linear nor logarithmic. Here, both viscous and Reynolds stresses are important, and steep velocity gradients cause turbulence production to reach a maximum.
} 


\subsection{Combined Wave-Current Flow}

Most corals exist in habitats where both current-driven steady flow and wave-driven oscillatory flow are important. The addition of an unsteady oscillatory component to the total velocity field can have significant implications for the structure and dynamics of the turbulence, as well as the resulting mixing rates and mass transport to the coral. Early evidence for wave enhancement of mass flux to corals was reported from a large-scale, fully enclosed self-sustaining mesocosm experiment, in which inorganic nutrient uptake, e.g., $\mathrm{PO}_{4}, \mathrm{NO}_{3}$, and $\mathrm{NH}_{4}$, was 2-3 times higher under wave forcing compared to equivalent steady flows 62]. Laboratory studies later supported and expanded upon this finding, reporting similar wave enhancement of mass transfer in idealized water flume experiments and suggesting that flow rate and substrate morphology/spacing significantly control the size of the effect 63, 64.

The major challenge of combined wave-current flow is the superposition of two unique turbulent boundary layers - a thin, oscillating wave boundary layer (WBL), and a thicker, steady current boundary layer (CBL) - that interact in a highly nonlinear fashion. During unsteady wave conditions, quantifying hydrodynamic parameters important for the estimation of mass flux can be problematic because of the manner in which large wave-induced velocity variances can contaminate calculations of velocity profiles 65], turbulent shear stresses [66, and friction velocities [67]. When wave motion is correctly accounted forthrough, for example, phase averaging or spectral separation - the turbulence structure of combined wavecurrent flow has been shown to differ significantly from steady wall turbulence. Characteristics of turbulence in wave-current flows exhibit a strong dependence on wave phase, with peak production, dissipation, and shear stress occurring in the decelerating phase 68. Time-dependent vertical profiles are altered due to the ejection of vortices during flow reversal, which are formed by the periodic acceleration of flow over bed roughness elements during periods of wave-induced adverse pressure gradients 69. These periodic "jets" are distinct, though difficult to distinguish, from traditional "bursts" of turbulent eddies in a steady flow, which arise from instabilities occurring over both smooth and rough beds. The result is that the interaction of a combined wave-current flow with the bed greatly enhances time-averaged bed shear stress and turbulence intensities compared to unidirectional flow alone.

\subsubsection{Linear Wave Theory}

Linear wave theory, also called Airy wave theory after $19^{\text {th }}$ century English mathematician George Biddell Airy, provides the equations that describe the fluid motion induced by surface gravity waves, such as the wind-driven waves typically found in coral reef environments. Governing equations for horizontal and vertical velocity components are defined in terms of several key wave parameters: wavelength $L$, wave period 
$T$, wave height $H$, and water depth $d$. The theory assumes inviscid, incompressible, irrotational fluid flow, and has been demonstrated to be valid for small ratios of wave height to water depth $(H: d)$ and wave height to wavelength $(H: L)$. The free-surface displacement $\eta$ of the wave field induces a pressure field, which drives subsurface fluid motion, and is given by

$$
\eta=\frac{H}{2} \cos (k x-\omega t)
$$

where $k=\frac{2 \pi}{L}$ is wavenumber and $\omega=\frac{2 \pi}{T}$ is angular wave frequency 70 . The instantaneous horizontal $u$ and vertical $w$ velocity components generated by the free-surface displacement are given by

$$
\begin{aligned}
& u=\frac{\omega H}{2} \frac{\cosh k(z+d)}{\sinh (k d)} \cos (k x-\omega t), \\
& w=\frac{\omega H}{2} \frac{\sinh k(z+d)}{\sinh (k d)} \sin (k x-\omega t),
\end{aligned}
$$

where $z$ is the vertical coordinate system, such that $z=0$ is the mean surface elevation and $z=-d$ is the ocean floor. The cos and sin terms in equations 2.10 and 2.11), respectively, indicate that the maximum horizontal and vertical velocity components of the subsurface fluid flow are $90^{\circ}$ out of phase, which creates the characteristic wave orbital motion. The magnitudes of wave orbital velocities decrease with increasing depth - as seen by the $(z+d)$ argument of the hyperbolic functions - and the shape of the wave orbitals flatten as they approach the seafloor, until very near the bed where the oscillatory wave motion becomes fully linearized to create a wave boundary layer. The size of the wave orbitals, or the horizontal distance of oscillatory motion at the bed, is described by the particle excursion length, which varies with elevation above the bed and is given as $A_{\max }(z)=u_{\max }(z) / \omega$.

\subsubsection{Wave-Current Boundary Layers}

The turbulence characteristics resulting from a thin, oscillating wave boundary layer are distinct from those resulting from a thick, steady current boundary layer. As noted above, when a current boundary layer with thickness $\delta_{c}$ is superimposed on a wave boundary layer with thickness $\delta_{w}$, the interaction between the two turbulence regimes is highly nonlinear, and a unique combined wave-current boundary layer is formed. Early pioneering measurements in a purely harmonic rough turbulent boundary layer were undertaken by Jonsson (1963) and later, within a combined wave-current boundary layer by Bakker and van Doorn (1978). These early experimental investigations focused primarily on obtaining mean velocity profiles, shear stresses, 
and bottom friction estimates 71,72 , and provided the basis for numerous analytical models of combined wave-current flow, such as those of Smith (1977), Grant and Madsen (1979), and Fredsøe (1984) 65, 67, 73. The theoretical models for combined wave-current flow predict the distribution of the mean current velocity profile to be logarithmic both inside and outside of the wave boundary layer, though with differing slopes, and each layer to generate a distinct friction velocity $-u_{* c}$ for the current-only region and $u_{* c w}$ for the combined region. The governing equations for mean current velocity $\bar{u}_{c}$ in a combined wave-current boundary layer over a rough surface are given by

$$
\begin{aligned}
& \bar{u}_{c}=\frac{u_{* c}}{\kappa} \frac{u_{* c}}{u_{* c w}} \ln \frac{30 z}{k_{b}} \quad z<\delta_{w}, \\
& \bar{u}_{c}=\frac{u_{* c}}{\kappa} \ln \frac{30 z}{k_{b c}} \quad \delta_{w}<z<\delta_{c},
\end{aligned}
$$

where $k_{b}$ is the physical bottom roughness and $k_{b c}$ is the "apparent" bottom roughness, which accounts for the effects of both physical roughness, as well as the drag generated by WBL turbulence and felt by the CBL. The magnitude of $k_{b c}$ has been shown to depend on the ratio of the particle excursion length to the physical bottom roughness $A_{\max } / k_{b}$, such that larger values of $A_{\max } / k_{b}$ generate a larger boundary layer thickness and consequently, a larger logarithmic layer [74.

\subsection{Canopy Flow}

Most previous work on the hydrodynamics of aquatic canopies has focused on large-scale systems such as seagrass meadows [75], kelp forests 76], and reef-scale coral canopies [77, while few studies have investigated the interaction of flow and algal canopies over corals, and to the author's knowledge, none have yet described the turbulent hydrodynamics, either in the lab or in situ. As a general feature of canopy flow, drag from the canopy top creates a sharp inflection in the mean velocity profile, resulting in increased levels of shear stress just above the canopy 78,79 . In unidirectional flow, turbulent stresses within the canopy are quickly damped as the canopy base is approached, and turbulence production rapidly shifts from shear-generation due to canopy top drag to wake-generation due to canopy element vortex shedding [80]. In oscillatory flow, however, the effect on turbulent stresses depends on the nature of the canopy structure, such that rigid, dense canopies, e.g., branching stony coral, enhance stresses within the canopy compared to above it [33], while flexible canopies, e.g., seagrasses and kelp forests, attenuate stresses and dampen vertical transport within the canopy compared to above it 81 . 
For corals covered by turf algae mats, the height of the algal canopy alters the thickness of the overlying momentum boundary layer as measured by mean flow profiles, with taller canopies increasing boundary layer thickness compared to shorter canopies [39]. Unidirectional flow speed has also been shown to control near-surface exchange processes for algal-covered corals with higher flow rates thinning vertical profiles of oxygen concentration and reducing the diffusive boundary layer thickness 82 . While an important role has been established for the impact of algal canopies on coral mass transfer, detailed measurements of turbulent hydrodynamics over algal-covered corals have not been made, and little is known about how turf algae mats modify the structure of the turbulent BBL, especially for combined wave-current flow environments in which corals are typically found.

\subsubsection{Mixing Layer Analogy}

Due to the lack of measurements of turbulent statistics over a coral algal canopy, it remains an open question as to the type of turbulent regime present in this system. Numerous measurements, however, have been made for a variety of terrestrial canopies, e.g., crops, forests, wind-tunnel models, and these results have led to the so-called mixing layer analogy for canopy flow. First described by Raupach, Finnigan, and Brunet in 1996 83], the mixing layer analogy argues that the active turbulence and coherent motions seen near a canopy top more closely resemble a plane mixing layer rather than a perturbed surface layer-the long-held previous model for canopy flow. Plane mixing layer turbulence forms when two co-flowing streams of different velocities interact along a mid plane, traditionally separated by a splitter plate in engineering schemes, but here represented by the presence of the canopy. The shear generated along the co-flowing streams induces Kelvin-Helmholtz instabilities, which propagate until fully developed turbulence is established.

The mixing layer analogy makes several predictions regarding key descriptors of turbulence in canopy flow, which are described below [78]:

1. The mean streamwise velocity profile $\bar{u}$ exhibits a sharp inflection at the canopy top, while second velocity moments, such as Reynolds shear stress $\overline{u^{\prime} w^{\prime}}$, fluctuating velocity standard deviations $\sigma_{u}=\left(\overline{u^{\prime 2}}\right)^{1 / 2}$ and $\sigma_{w}=\left(\overline{w^{\prime 2}}\right)^{1 / 2}$, and the correlation coefficient $r_{u w}=\overline{u^{\prime} w^{\prime}} /\left(\sigma_{u} \sigma_{w}\right)$, display vertical inhomogeneity and rapid decay within the canopy.

2. A roughness sublayer' exists from $z=h$ to approximately $z=2 h$, where $h$ is the canopy height, in which values of $\sigma_{u} / u_{*}, \sigma_{w} / u_{*}$, and $r_{u w}$ vary significant from their surface layer values. Canopy flow values of $\sigma_{u} / u_{*}$ and $\sigma_{w} / u_{*}$ decrease from 2.5 to 2.0 and 1.25 to 1.0 , respectively, and $r_{u w}$ decreases from -0.32 to -0.5 , indicating a more efficient transfer of momentum near a canopy top compared to a surface layer. Additionally, eddy diffusivity for momentum, $\nu_{e}=-\overline{u^{\prime} w^{\prime}} /(\partial \bar{u} / \partial z)$, is enhanced between $10-50 \%$ in this 
region.

3. The turbulent kinetic budget shifts from a local equilibrium state typical of inertial sublayer turbulence, in which shear production and viscous dissipation are balanced, to a regime in which turbulent and pressure transport terms approach magnitudes similar to production terms, indicating highly nonlocal turbulence.

4. The turbulent length scale for canopy flow is set by the strength of the shear at the canopy top, i.e., $\ell_{s}=\bar{u}(h) /(\partial \bar{u}(h) / \partial z)$. The size of $\ell_{s}$ decreases as the magnitude of the shear increases and exhibits a dependence on canopy density. By contrast, the turbulent mixing length scale for surface layer turbulence scales with distance from the wall, according to $\ell_{m}=\kappa z$.

The mixing layer analogy predicts additional differences in $u^{\prime}$ and $w^{\prime}$ skewness as well as the structure of coherent motions; however, these properties are less relevant for questions of coral mass transfer considered in this dissertation. The above hypotheses have been well demonstrated for terrestrial canopies under unidirectional flow $78,83,84$, but no measurements have been made to determine if the analogy - and its predictive capabilities - apply to aquatic systems subject to oscillatory flows.

\subsection{Large- and Small-Scale Morphology}

From a (relatively) large-scale hydrodynamic perspective, fluid motion past a massive lobate coral represents flow past a bluff body. A stationary body exposed to moving fluid experiences a drag force composed of two components: pressure (or form) drag, which derives from wake-induced eddy production that creates a pressure loss on the downstream side, and frictional (or viscous) drag, which results from the interaction of the moving fluid along the surface of the body creating a shear stress. An object is considered to be a bluff body if, at high Reynolds numbers, the total drag force is dominated by pressure drag. Early experiments demonstrated that adding roughness elements to the surface of a sphere decreased the critical Reynolds number for transition to turbulence, thereby reducing total drag [85]. Subsequent work explained the mechanism behind this effect, showing that the ejection of momentum from roughness elements into the overlying boundary layer acts in opposition to the adverse pressure gradient and delays flow separation [86]. With regard to the analogous impact on coral mass transfer, the biological implications of treating a coral as a bluff body arise from: 1) the pressure distribution across the coral's surface and the effect on boundary layer separation, 2) the enhancement of turbulence-mediated mixing resulting from eddy shedding, and 3) the control over diffusive boundary layer thickness by friction- and pressure-induced shear stress. 

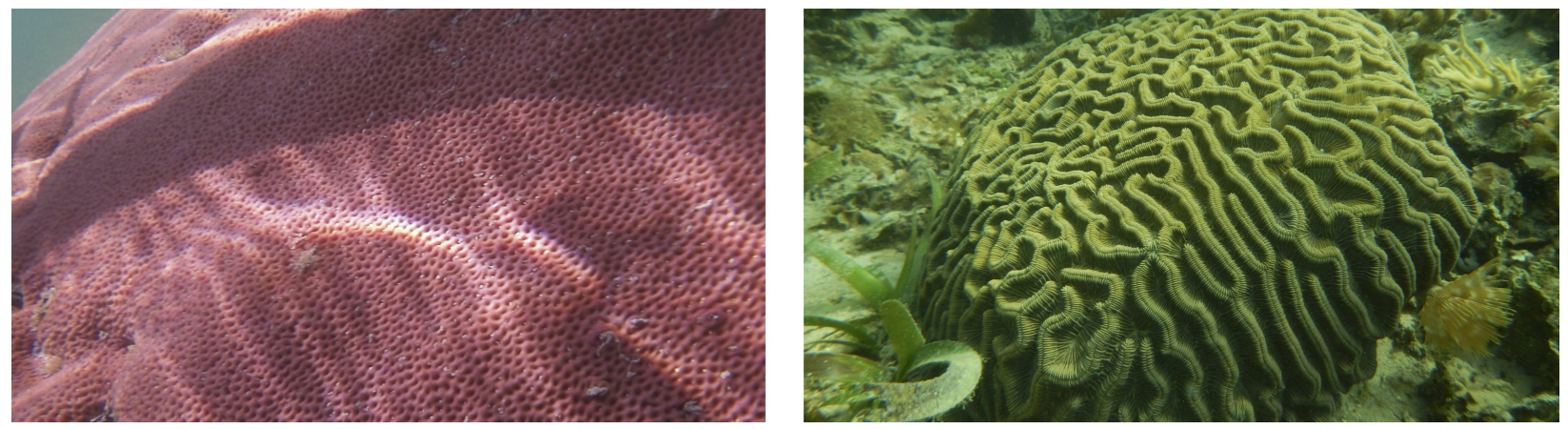

Figure 2.2: Close-up photographs of the relatively smooth surface of a Siderastrea siderea coral (left) and the highly rugose surface of a Colpophyllia natans coral (right).

\subsubsection{Surface Roughness}

Corals, however, are not simply idealized, smooth bluff bodies. Rather, corals exhibit a variety of small-scale surface morphologies that can impact surface layer hydrodynamics, ranging from the lightly dimpled surface of Siderastrea siderea to the highly convoluted surface of Colpophyllia natans (see figure 2.2). For corals with roughness heights that exceed the concentration boundary layer thickness (typically on the order of tens to hundreds of $\mu \mathrm{m}$ ), mass transfer rates have been shown to scale linearly with total surface area (TSA) 37]. This finding implies that all surfaces are exposed comparably to the flow conditions, a reasonable assumption provided roughness-generated turbulence transports momentum into less exposed areas (which may not be true for the cryptic regions of branching corals). Additionally, if mass transfer rates depend on total surface area, then they must also be a function of the coral's rugosity, i.e., the ratio of total surface area to projected planar area (PPA), $R u=$ TSA/PPA. Rugosity is a widely used measure of the "roughness" of surface topography, common in fields such as oceanography, ecology, and materials sciences.

Though an object's surface rugosity has been shown to affect the mass flux rate, the individual roughness element size and distribution also plays a key role in altering local hydrodynamics and thus, scalar transport. Historically, two-dimensional "bar" roughness has been a common idealized system used to investigate surface roughness effects on the turbulent boundary layer and serves as a reasonable analogue for the ridges present on lobate brain corals like C. natans pictured above. Perry et al. (1969) defined two types of bar roughness based on the ratio of the distance between elements $w$ to the element height $k$, i.e., the $w / k$ ratio 87 . For $w / k<1$, the roughness is known as "d-type" (the $\mathrm{d}$ originally referred to pipe diameter), and is characterized by stable, fully contained flow within the roughness cavity. For $w / k>1$, the roughness is known as "k-type" (the k originally referred to the roughness scale), and is characterized by periodic vortex shedding from the roughness cavity that imparts momentum into the boundary layer. Figure 2.3 illustrates the differences in dimensions between the two types. Recent direct numerical simulations have 


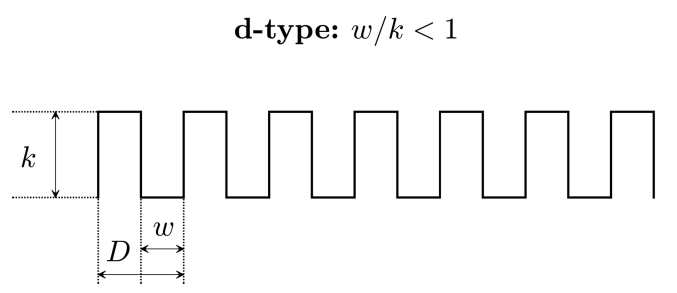

k-type: $w / k>1$

Figure 2.3: Idealized d-type (left) and k-type (right) bar roughnesses.

shown that the distinction between d-type and k-type roughness is more accurately reflected by the relative contributions of frictional and pressure drag, such that d-type behavior occurs when friction dominates and k-type behavior occurs when pressure dominates [38].

\subsubsection{Roughness Length Scale and Roughness Function}

The degree to which roughness elements protrude through the viscous sublayer and disrupt its formation can be described by the normalized roughness scale, $k_{b}^{+}=k_{b} u_{*} / \nu$ (also known as the roughness Reynolds number) and divided into three regimes according to 88

$$
\begin{aligned}
\text { hydraulically smooth : } & 0 \leq k_{b}^{+} \leq 5, \\
\text { transitional region : } & 5<k_{b}^{+}<70, \\
\text { fully rough : } & 70 \leq k_{b}^{+} .
\end{aligned}
$$

For fully rough flow, the viscous sublayer is completely disturbed and the scaling of the logarithmic layer with viscosity, as in the smooth-wall case of equation 2.8, is no longer valid. Instead, the velocity distribution of the overlap layer is a function only of height $z$ and the nature of the surface roughness, such that

$$
\frac{\bar{u}}{u_{*}}=\frac{1}{\kappa} \ln \frac{z}{z_{0}}
$$

where $z_{0}$ is the roughness length scale, which in the fully rough limit, depends only on the geometry of the surface roughness [80]. Historically, the value of $z_{0}$ was first described for homogenous sand roughness (as studied by Nikuradse in 1933) and found to equal $k_{b} / 30$ [89]; however, for a surface topography with such diverse geometric features as lobate corals, a relationship between roughness height $k_{b}$ and roughness length scale $z_{0}$ has not yet been determined.

The ratio of roughness length scale to roughness height, i.e., $z_{0} / k_{b}$, represents the depth of momentum absorption by the surface roughness, and is analogous to the roughness function $\Delta u / u_{*}$ used in engineering 
approaches to surface roughness. The engineering version of the modified log law for rough surfaces is given by

$$
\frac{\bar{u}}{u_{*}}=\frac{1}{\kappa} \ln \frac{u_{*} z}{\nu}+C_{0}-\frac{\Delta u}{u_{*}} .
$$

The roughness function reflects the downward shift of the mean velocity profile relative to a smooth wall and can be converted to the roughness length scale according to $\Delta u / u_{*}=C_{0}+\kappa^{-1} \ln \left(z_{0} u_{*} / \nu\right)$, where $C_{0}=5.1$ for a smooth wall 87 .

\subsection{Mass Transfer}

The advection-diffusion equation governs the transport of a passive conservative scalar $c$ subject to both diffusive and convective motion and is given in tensor notation by

$$
\frac{\partial c}{\partial t}=\nabla \cdot(D \nabla c)-\nabla \cdot\left(u_{i} c\right)+S
$$

where $D$ is the molecular diffusivity and $S$ is a source or sink of the scalar. As in the derivation of the mean momentum equation 2.4], the instantaneous scalar concentration can be decomposed into a mean component and fluctuating component, respectively, such that $c=\bar{c}+c^{\prime}$. Assuming a constant diffusivity $D$, the absence of any sources or sinks, and stationary, steady-state concentration levels (a reasonable assumption given that the bulk ocean concentration can be considered invariant on the coral-scale), then the Reynolds-averaged advection-diffusion equation becomes

$$
\bar{u}_{i} \frac{\partial \bar{c}}{\partial x_{i}}=-\frac{\partial}{\partial x_{i}}\left(\overline{u_{i}^{\prime} c^{\prime}}\right)+D \frac{\partial^{2} \bar{c}}{\partial x_{i} \partial x_{i}}
$$

where the terms on the left-hand-side represent scalar advection, and the terms on the right-hand-side represent turbulent diffusion and molecular diffusion, respectively. For mass flux across a surface, modeling efforts typically assume stationarity in the streamwise and lateral directions, i.e., $\partial \bar{c} / \partial x=0$ and $\partial \bar{c} / \partial y=0$, and as with mass transport to a coral, for example, vertical mixing is the dominant measure of interest. Given this assumption of a 2-dimensional boundary layer, the vertical turbulent flux component, i.e., $\overline{w^{\prime} c^{\prime}}$, can be modeled in analogous fashion to the Boussinesq eddy viscosity hypothesis, yielding

$$
\overline{w^{\prime} c^{\prime}}=\varepsilon_{M} \frac{\partial \bar{c}}{\partial z}
$$


where $\varepsilon_{M}$ is the eddy diffusivity for mass (similar to the mixing length $\ell_{m}$ used to describe the relationship between the Reynolds stress and mean velocity gradient, $\overline{u^{\prime} w^{\prime}}=\ell_{m} \frac{\partial \bar{u}}{\partial z}$.

Much like the dimensionless Reynolds number describes the relative influence of inertial to viscous forces for fluid flow, several dimensionless parameters are used to describe the relative influence of forces in mass and heat transfer. The ratio of eddy viscosity of momentum to eddy diffusivity of mass defines the turbulent Schmidt number, $S c_{t} \equiv \nu_{e} / \varepsilon_{M}$, which is the turbulent version of the regular Schmidt number relating viscous diffusion to molecular diffusion, $S c \equiv \nu / D$. Since the dimensionless governing equations for heat and mass transfer are of the same functional form, simulations of mass transfer across a coral surface can be accomplished by first modeling heat transfer, then relating the eddy diffusivity for heat $\varepsilon_{H}$ to the eddy diffusivity for mass in order to estimate mass flux. The ratio of mass to heat eddy diffusivities defines the turbulent Prandtl number, i.e., $P r_{t} \equiv \varepsilon_{M} / \varepsilon_{H}$, which is the turbulent version of the regular Prandtl number relating viscous diffusion to thermal diffusion $\operatorname{Pr} \equiv \nu / \alpha$. If $\operatorname{Pr} r_{t}=1$, known as the Reynolds analogy, then the relationship between heat and mass transfer is exact. However, empirical results show that $P r_{t}$ depends on the specific fluid and typically ranges between 0.7 and 0.9 90.

Recalling the previous discussion on benthic boundary layers and concentration gradients, the diffusive boundary layer at the coral surface, which acts as a bottleneck in nutrient uptake, creates a mass-transfer limited system for corals [22, 91]. That is, corals utilize nutrients as quickly as they obtain them from the water column (as opposed to a biologically limited system, in which the reaction kinetics of corals can not process nutrients as quickly as they are provided). Since the DBL controls access to the coral surface, the rate of mass transfer $J\left(\mathrm{~mol} \mathrm{~m}^{-2} \mathrm{~s}^{-1}\right.$, where $\mathrm{m}^{-2}$ represents the projected planar area) can be parameterized according to scalar concentrations in this region, given by

$$
J=S\left(c_{b u l k}-c_{\text {surf }}\right)
$$

where $S$ is the mass transfer coefficient $\left(\mathrm{m} \mathrm{s}^{-1}\right), c_{b u l k}\left(\mathrm{~mol} \mathrm{~m}^{-3}\right)$ is the bulk nutrient concentration of the water column, and $c_{\text {surf }}\left(\mathrm{mol} \mathrm{m}^{-3}\right)$ is the nutrient concentration at the coral surface, assumed to equal 0 for a mass-limited system that fully consumes available nutrients. The mass transfer coefficient across the diffusive boundary layer can be measured directly according to

$$
S=\frac{-D(\partial \bar{c} / \partial z)}{c_{\text {surf }}-c_{b u l k}}
$$

which when substituted into equation $(2.22)$, yields the diffusive version of the turbulent mass flux equation (2.21) that governs mixing in the turbulent boundary layer above the diffusive region. 
Given that the molecular diffusivity, surface concentration, and bulk concentrations are all typically constant, the only way to enhance mass transfer across the DBL is to steepen (or disrupt entirely) the gradient $\frac{\partial \bar{c}}{\partial z}$. A reduction of DBL thickness can be accomplished by increasing boundary layer turbulenceeither enhancing turbulent shear stresses or the prevalence of coherent motions, e.g., sweeps and ejections 92] - or by increasing the free-stream velocity 91. The ratio of the mass transfer coefficient to the advection velocity $u_{\infty}$ determines how efficient a system is at extracting nutrients from the available passing water, and is called the mass Stanton number, $S t_{m}=S / u_{\infty}$, (the mass equivalent to the regular Stanton number describing heat transfer under forced convection) 93. The Stanton number for mass has been shown to be a function of Reynolds number, Schmidt number, and surface roughness/geometry 27]. Additionally, wave motion can have a significant impact on mass transfer, generating 2-3x higher fluxes compared to comparable unidirectional flows [62], and in field experiments, coral nutrient uptake rates measure 40x higher than predicted from engineering estimates [27, likely due to wave surge, higher rugosity values for corals compared to measured engineering geometries, and active ciliary micro-mixing at biotic surfaces [25]. However, the effect on total mass transfer - and associated uptake efficiency rates - due to combined d-type and k-type roughness on a bluff body exposed to oscillatory flow has not been investigated. 


\section{Chapter 3}

\section{Structure and Dynamics of Turbulent Boundary Layer Flow Over Healthy and Algae-covered Corals}

Note: The contents of this chapter have been published in the journal Coral Reefs on 2 April 2016 and are presented here in a slightly modified format. The citation is: Stocking, J.B., Rippe, J.P. \& Reidenbach, M.A. Coral Reefs (2016) 35: 1047. doi:10.1007/s00338-016-1446-8 [94].

\subsection{Introduction}

As sessile, benthic organisms, corals depend on mass transfer to and from the overlying water column for many biologically necessary processes, including photosynthesis by endosymbiont zooxanthellae, carbonate skeleton building, larval dispersal and settlement, and nutrient and dissolved gas exchange 28 , 30. The mass transfer required for these processes can be achieved through diffusion alone, but even small amounts of fluid advection can greatly enhance the efficiency of mass flux 31. Corals are typically found in shallow, oligotrophic tropical and sub-tropical coastal oceans, in which fluid motion generated by both currents and waves supplies the momentum required for mass-exchange processes. The dominant factor controlling the vertical transport of mass between the water column and coral surface is the fine-scale hydrodynamics of the turbulent bottom boundary layer (BBL) adjacent to the coral surface 33 .

Within coral reef communities, many other organisms directly compete with corals for resources in the nutrient-poor marine habitat, and as a result, can alter BBL dynamics. In particular, benthic macroalgae 
often exhibit a competitive advantage for space and light under conditions of limited herbivory or excessive nutrient loading [56]. Algal turfs impact boundary layer hydrodynamics by altering the mean velocity profile [39], in addition to direct interactions such as overgrowth and shading, chemical attack, and surface abrasion 17]. Little is known, however, about how algae affects the turbulence regime within the BBL, which has important implications for vertical transport and mixing between the coral and overlying water columncritical processes for coral mass transfer, but broadly applicable to other substrates with algal canopies as well.

\subsubsection{Hydrodynamics of the Turbulent Boundary Layer}

Along the surface of an individual coral, the overlying water column interacts through friction with the fixed coral boundary, producing a layer of shear flow that generates a turbulent wall boundary layer 95]. The BBL is composed of: 1) an inner layer very near the wall, in which fluid viscosity is the controlling scale and which typically extends a few hundred microns in elevation, and 2) an outer layer in which the flow resembles inviscid, wall-free turbulence and whose vertical range depends on the scale of the maximum size of turbulent eddies [61]. Connecting these two regions is an overlap layer, called the inertial sublayer, which typically measures $15-20 \%$ of the total BBL thickness and where inertial effects dominate, causing the velocity to follow a logarithmic profile - a key characteristic of wall-bounded shear flow.

Close to the coralwater interface within the inner layer, fluid viscosity dampens turbulent motions, and molecular diffusion is the primary mechanism of vertical transport. The production or consumption of dissolved materials by the coral surface creates a concentration gradient, forming a diffusive boundary layer (DBL). Typically less than $1.0 \mathrm{~mm}$ thick, the size of the DBL is controlled by surface roughness, flow speed, and the molecular diffusivities of relevant dissolved materials (e.g., $\mathrm{O}_{2}, \mathrm{CO}_{2}, \mathrm{PO}_{4}^{3-}, \mathrm{NH}_{4}^{+}$). Ultimately, the DBL governs the rate of mass flux to and from individual coral polyps and acts as a bottleneck for mass transfer [48. Increases in wall shear stress $\tau_{\text {wall }}$ and in turbulent kinetic energy $q^{2}$ reduce the thickness of the diffusive boundary layer and can enhance mass flux across this water-substrate interface [49, 53. Further, corals themselves may actively disrupt the DBL, by employing ciliary beating to generate microscale vortical flows, which enhance mass transfer up to 4-fold compared to molecular diffusion alone 25].

Above the DBL, vertical mass transport is primarily controlled by turbulent diffusivity $\nu_{e}$, the magnitude of which is proportional to the ratio of turbulent kinetic energy $q^{2}$ to the turbulent dissipation rate $\epsilon$, i.e., $\nu_{e} \propto\left(q^{2}\right)^{2} / \epsilon$ 61]. Higher levels of turbulent kinetic energy (TKE), therefore, act to mix and transport both dissolved and particulate matter above the coral surface more efficiently. Additionally, increases in turbulence intensity - defined as the ratio of turbulent fluctuations to the mean free-stream velocity — have 
been shown to enhance mass transfer rates 96 . Of interest for this study are the biological implications for coral health due to the turbulent boundary layer, and thus, turbulent control on diffusive and convective mass transport processes.

\subsubsection{Boundary Layer Alterations Due to Waves and Canopies}

Coral reef ecosystems are generally exposed to a combination of tide-driven unidirectional currents and wave-driven oscillatory motion 97 . Combined wave-current flows feature a non-linear interaction between the steady turbulent boundary layer created by the unidirectional current and the thin, time-varying wave boundary layer created by the oscillatory flow 67]. The time-averaged velocity profile for combined wave-current flow has been shown to be logarithmic within and above the wave boundary layer, though with different slopes for each region [65]. The presence of waves significantly impacts reef biophysical processes, e.g., enhancing vertical flow rates and mass flux within branching corals 64.

Additionally, the presence of a surface canopy can significantly alter boundary layer hydrodynamics, evident by a sharp inflection in velocity at the canopy top which results in increased levels of shear just above the canopy 83. For unidirectional flow, turbulent stresses within the canopy quickly dampen as the canopy floor is approached 78. Under oscillatory forcing, canopy structure is important, such that rigid canopies generate larger turbulent stresses in the upper canopy compared to above the canopy 33 , whereas flexible canopies dampen turbulent stresses and vertical transport within the canopy compared to above it 81 .

Investigations into algal canopy flows have measured mean flow quantities, finding that higher canopies extend the vertical range of the overlying momentum boundary layer compared to lower canopies 39]. Unidirectional flow speed has also been shown to control vertical profiles of oxygen concentration over algae-covered corals, with higher flow rates reducing the DBL thickness [98], thereby demonstrating a key role for algal canopies in controlling coral mass flux. More broadly, reef algal turfs make important contributions in their own right to benthic primary production, which sustains higher trophic levels in reef food webs. Understanding the turbulent hydrodynamics of algal canopy flow has wide applicability; however, detailed measurements of turbulence characteristics over algae-covered corals have not been made, and little is known about how algal turfs may modify the structure and type of boundary layer turbulence, especially for combined wave-current flow environments.

The purpose of this study is to provide a detailed in situ characterization of the fine-scale turbulent boundary layer dynamics for both healthy and degraded, algae-covered coral exposed to naturally occurring wave-current flows. This study seeks to answer the following questions:

1. What are the hydrodynamic characteristics - specifically mean velocity, bed shear stress, Reynolds 
stress, turbulent kinetic energy, and turbulent production and dissipation-that describe the boundary layer at a coral-water interface under combined wave-current conditions?

2. How does an algal turf canopy alter the structure and dynamics of the turbulent boundary layer, and what are the implications for mass transfer at the coral-water interface?

Using a combination of an in situ particle image velocimetry (PIV) system and a profiling acoustic Doppler velocimeter (ADV), boundary layer flow profiles were recorded over the scleractinian coral Siderastrea siderea in the coastal Caribbean waters of Bocas del Toro, Panamá.

\subsection{Methods}

\subsubsection{Study Site}

Experiments were conducted on the fringing coral reef in the Caribbean archipelago of Bocas del Toro, Panamá $\left(9^{\circ} 21 \mathrm{~N}, 82^{\circ} 16 \mathrm{~W}\right)$. The study site (figure 3.1) is situated near the Smithsonian Tropical Research Institute (STRI) approximately $50 \mathrm{~m}$ off the bayside shore of Isla Colón. The reef begins just below the mean low tide mark and extends to depths $>20 \mathrm{~m}$. The reef composition is dominated by the branching coral Porites furcata and the lobate (brain) corals Colpophyllia natans and S. siderea, with a variety of sponges, anemones, and urchins also common. Measurements were collected over healthy and algae-covered S. siderea corals (Fig. 2a, b) situated at depths of approximately 1.0-2.5 m during two site visits: 7-20 June 2012 and

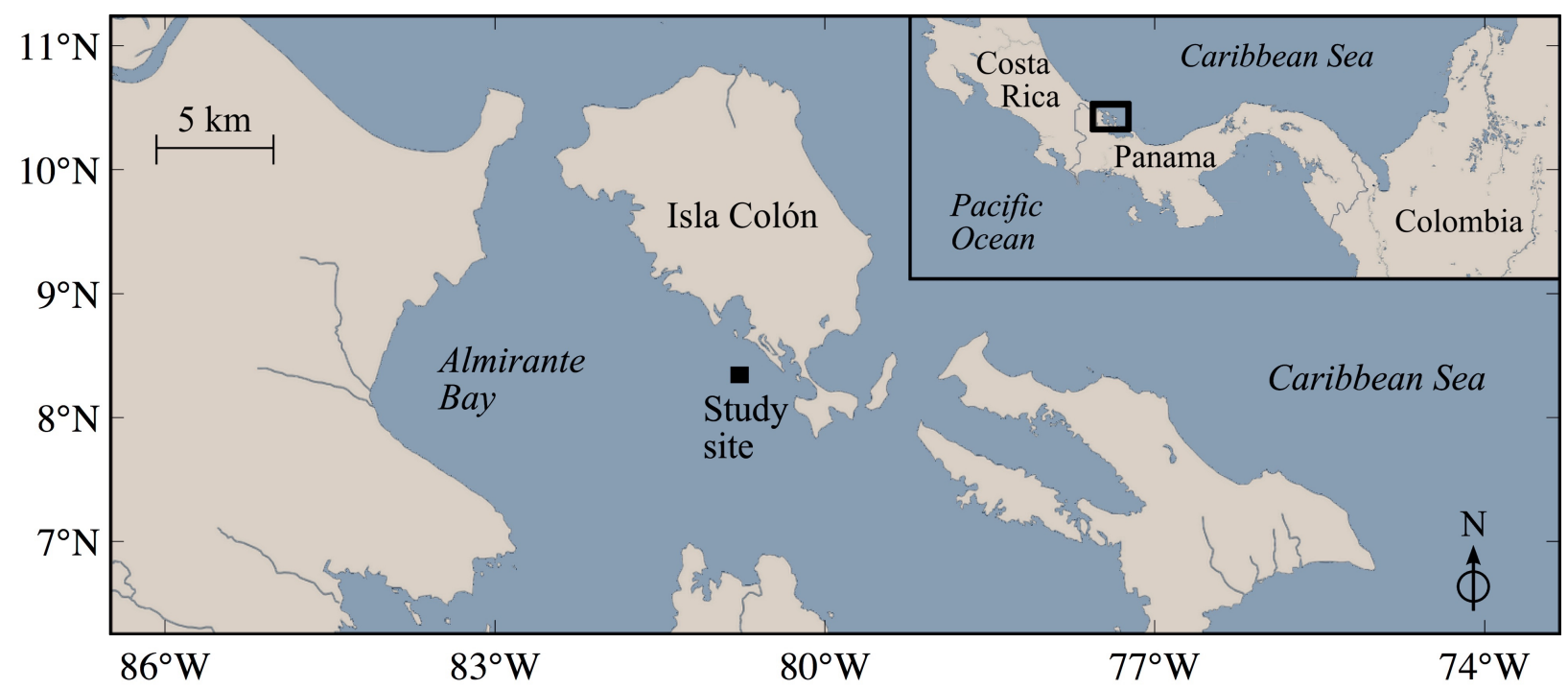

Figure 3.1: Site map of island archipelago of Bocas del Toro, Panamá. The study site is located on landward side of Isla Colón. 
12-30 July 2013. The site is exposed to mixed semidiurnal tides with a tidal range of approximately $0.5 \mathrm{~m}$ 99. The wave environment is primarily wind-driven from the southeast, with mean winds of $1.9 \mathrm{~m} \mathrm{~s}^{-1}$ during June 2012 and $1.8 \mathrm{~m} \mathrm{~s}^{-1}$ during July 2013.

\subsubsection{Coral and Algal Morphology}

The boulder-shaped species S. siderea was selected because of its prevalence at the study site, uniform surface topography, and susceptibility to algal overgrowth. Polyps of sampled corals measured approximately $0.25 \mathrm{~cm}$ in height, creating a slightly dimpled surface at the coral-water interface. During the day when all measurements were collected, polyp tentacles were retracted so that a relatively uniform roughness height could be estimated. Measurements were taken as close as possible to the center of the corals to reduce the effects of large-scale topographic features that might generate leading or trailing edge effects, and efforts were made to minimize the influence of non-local turbulence from surrounding structures by selecting spatially isolated corals and aligning the instrument frame with the dominant flow direction (see figure 3.2). The influence of non-local turbulence, however, has been shown to be relatively limited, enhancing mass transfer coefficients up to only $\pm 10 \%$ for very high levels of free-stream turbulence 96.

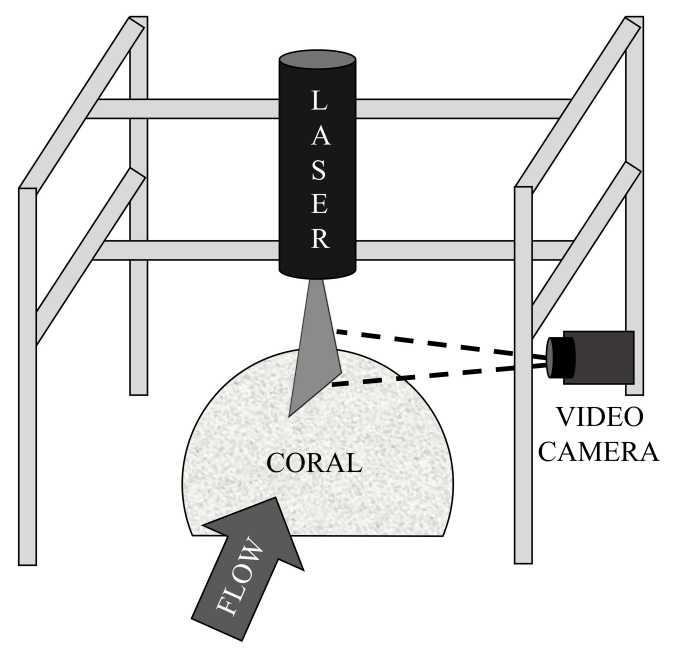

Figure 3.2: Schematic of instrument frame with particle image velocimetry experimental setup over healthy Siderastrea siderea coral. The frame was aligned with dominant flow direction to avoid non-local turbulence from frame legs. Acoustic doppler velocimeter (ADV) measurements were obtained using the same frame setup with the ADV instrument replacing the laser. 


\subsubsection{Instrumentation}

Siderastrea siderea corals with algal turfs, which colonized the surface in discrete patches, were selected in close proximity to the healthy corals. Polyps beneath the canopy were degraded, but likely in a still recoverable state. The algae found in the Bocas del Toro reef, common among Caribbean marine habitats, is a multispecies assemblage of green, red, and brown seaweeds with filamentous cyanobacteria 57]. Estimates of algal canopy density $\lambda$ were made based on the total algal frontal area per unit ground area 83, given as $\lambda \equiv \frac{h d}{A / n}$, where $h$ is the canopy height, $d$ is the algal stem diameter, $A$ is the ground area, and $n$ is the number of stems in the measured area. Typical values for canopy parameters were estimated as $h \sim 10 \mathrm{~mm}, d \sim 0.2 \mathrm{~mm}, A \sim 100 \mathrm{~mm}^{2}$, and $n \sim 20$, which yields a canopy roughness density of $\lambda \sim 0.4$, significantly higher than the threshold of $\lambda>0.1$ that defines a "dense" vegetation canopy [79].

Three-component velocity measurements (horizontal $u$, lateral $v$, and vertical $w$ ) were collected using a profiling acoustic Doppler velocimeter (Vectrino Profiler, Nortek AS) at a sampling rate of $25 \mathrm{~Hz}$ for burst intervals ranging between 5 and 10 min (7,500-15,000 samples per burst). The minimum burst length was set to allow for a balance between obtaining convergence of mean velocity and turbulent quantities while still maintaining stationarity within changing wave and tidal conditions [100. Burst intervals were rotated into the dominant streamwise direction $u$ during post-processing. Stationarity of the tidal current for each burst was verified using the nonparametric reverse arrangements test $(N=10, \alpha=0.05)$ [101], and bursts exhibiting non-stationarity were omitted. Figure 3.3 plots the full instantaneous velocity record for a sample

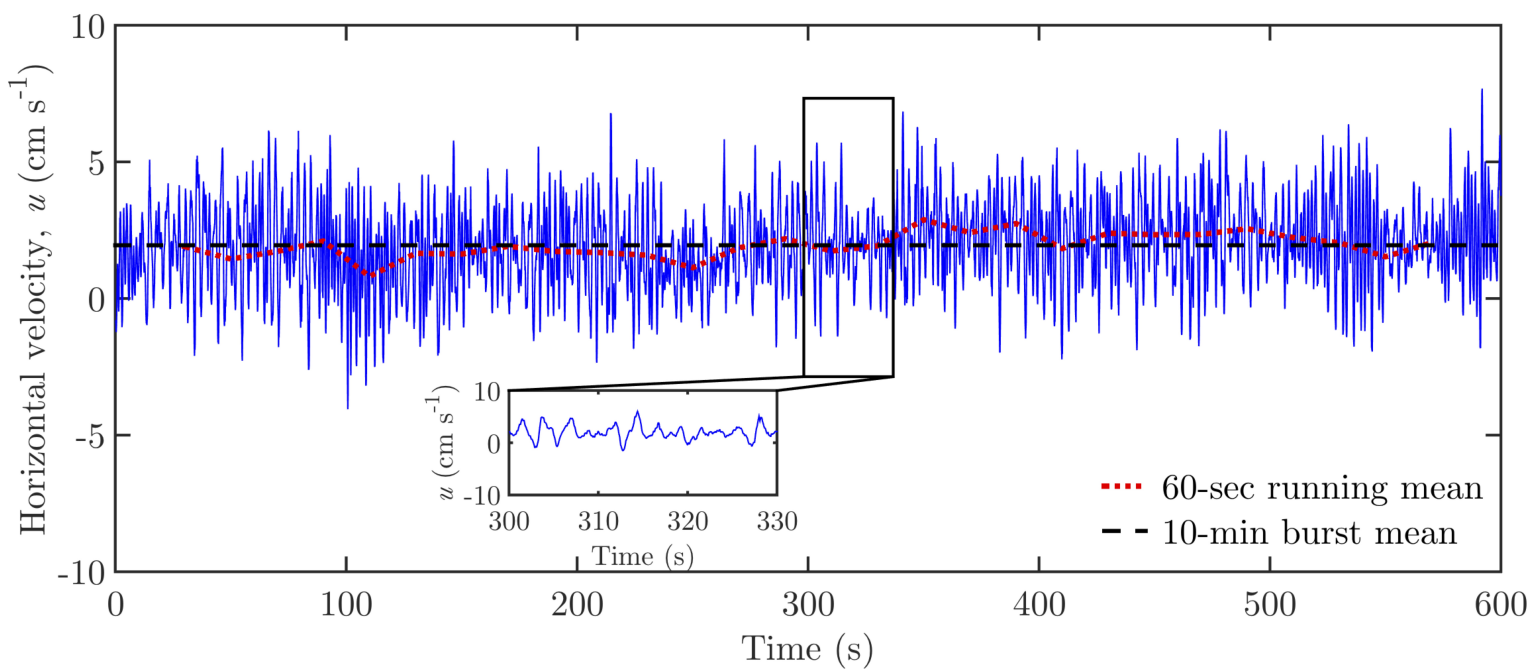

Figure 3.3: Typical record of instantaneous horizontal velocity $u$ for a 10-min burst. Black dashed line shows background mean, i.e., tidal current velocity, over entire record. Red dotted line plots 60-s running mean and shows consistent stationarity about the burst mean, confirmed quantitatively using reverse arrangements test. Inset plot expands a 30-s segment of the burst to illustrate the spectral wave signal with dominant period of $2.7 \mathrm{~s}$. Data taken from a single ADV measurement bin $\sim 1.5 \mathrm{~cm}$ above healthy coral surface. 

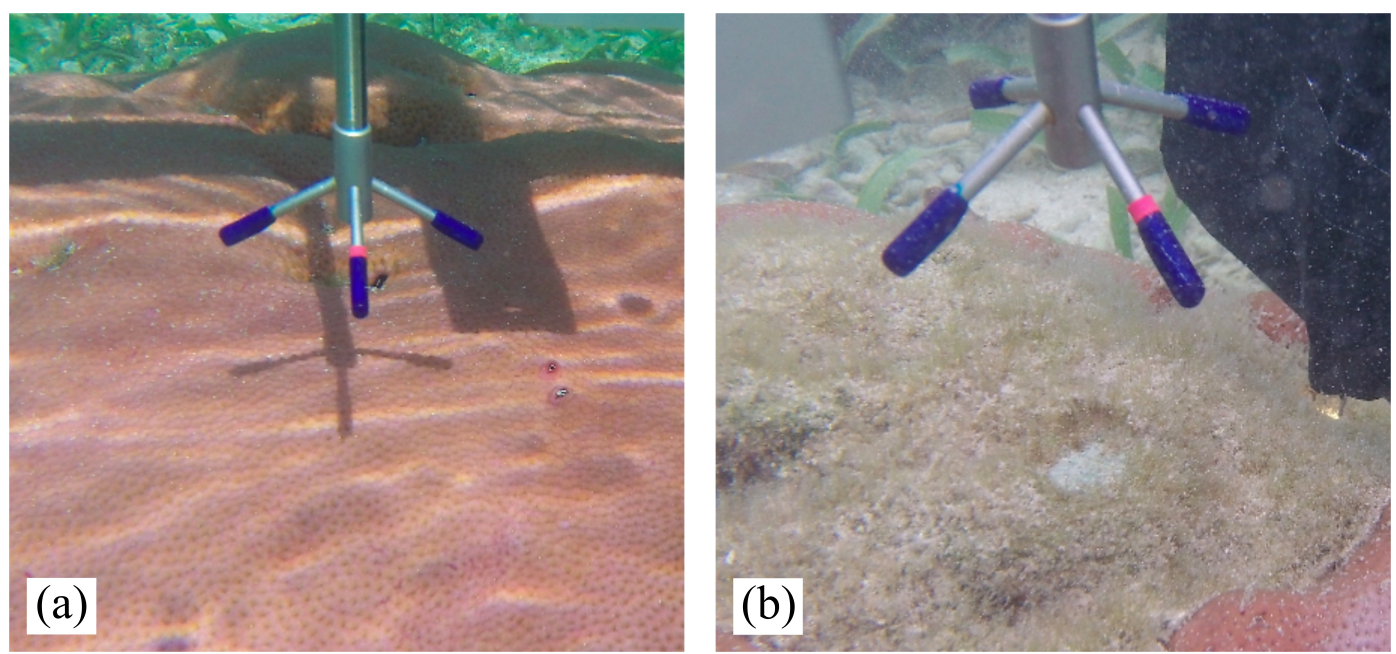

Figure 3.4: (a) Vectrino acoustic Doppler velocimeter probe tip positioned above a healthy Siderastrea siderea coral. (b) Probe tip positioned above algae-covered S. siderea coral. Note white patch of bleached coral exposed after algae removal for in-canopy flow measurements.

10-min burst and illustrates the stationarity of the background current. The instrument was mounted to an aluminum frame with a width and length of $1 \mathrm{~m}$ and height-adjustable legs used to minimize instrument tilt. Velocity profiles approximately $3 \mathrm{~cm}$ high with a resolution of $1 \mathrm{~mm}$ were obtained over the coral surface (see figure 3.4 ). For the algae-covered corals, a small patch $\left(\sim 1 \mathrm{~cm}^{2}\right)$ of algae directly beneath the probe tip was cleared to allow for within-canopy measurements (see figure $3.4 \mathrm{~b}$ ). To accommodate the double-averaging technique typically used to describe canopy flows 102 , multiple spatial measurements of time-averaged profiles were collected over the same and different corals. Though simultaneous spatial measurements were not possible due to the limited space in the canopy patch, normalization of multiple spatial profiles by the free-stream velocity (described below) allowed for a single double-averaged profile to be computed.

As an instrument class, acoustic Doppler velocimeters (ADVs) have known difficulties measuring near boundaries due to interference of the reflected acoustic pulses echoing from solid boundaries 103. The height above the boundary for which echoes bias velocity estimates can be identified by a sharp increase in the signal-to-noise ratio (SNR) and depends on boundary material and flow conditions 104. We measured this contamination height for each burst by identifying the transition from decreasing to increasing SNR derived from the mean of the four transducer heads. The location of the coral surface was determined by the instruments bottom detection measurement and confirmed by steady zero velocity components. Together, these two locations determined the height of echo interference, which extended $\sim 3 \mathrm{~mm}$ above the coral surface and is indicated by a gray box in all plots of ADV data.

A second set of independent velocity measurements used to obtain spatial information of velocity structure along the coral surface was made using a custom-fabricated underwater particle image velocimetry 
(PIV) system. A 532-nm green laser (300 mW Hercules Series, Laserglow Technologies) was secured inside a watertight housing and directed through a convex lens at the housing base to create a continuous, twodimensional sheet of laser light. The laser housing was mounted on the instrument frame above the coral and aligned with the dominant flow direction. Perpendicular to the laser sheet, an underwater video camera (Sony HDR-CX160, Sony Electronics Inc.) was attached to the frame and fitted with a $530 \pm 3 \mathrm{~nm}$ bandpass filter (Andover Corporation) to remove excess daylight. The camera recorded a field-of-view of approximately $5 \times 6 \mathrm{~cm}$ at 30 frames $\mathrm{s}^{-1}$. Free-floating particulates in the water column served as velocity tracers and were illuminated and recorded as they passed through the laser sheet plane. PIV measurements were only performed over the healthy coral since the algal canopy blocked the field-of-view of the camera. Raw PIV video records were processed using open-source MatPIV 1.6.1 software written for MATLAB [105, which implements a hybrid digital PIV technique using iterative windowing to a final interrogation resolution of $32 \times 32$ pixels. Outliers were identified using global mean and local median filters $( \pm 3$ and \pm 2.5 standard deviations, respectively), and replaced via linear interpolation. Accuracy of PIV measurements was previously determined in a tow-tank to be $\pm 6 \%$ of the mean velocity magnitude 106 .

\subsubsection{Analysis of Turbulence Statistics}

In combined wave-current flow, wave orbital motions outside the thin wave boundary layer are irrotational and do not contribute to the shear-generated turbulence from the current. However, wave orbitals induce large variances in the horizontal and vertical velocity components, which mask velocity variances due to turbulence, making it necessary to separate the wave and turbulence parts of the signal. For wave-current flows, the components of the total instantaneous velocity $u$ can be separated via Reynolds decomposition according to

$$
u=\bar{u}+\widetilde{u}+u^{\prime}
$$

where $\bar{u}$ is the mean current velocity, $\widetilde{u}$ is the instantaneous wave orbital velocity, and $u^{\prime}$ is the instantaneous turbulent velocity. Wave and turbulence perturbations were separated using a spectral decomposition technique called the Phase Method [107, which converts the temporal velocity record into frequency space via Fourier transformation and isolates the wave stress (evident as the clear wave peak in the sample power spectrum of figure 3.5 using the phase lag between horizontal and vertical components of the two-sided cross-spectral density. Details of the Phase Method can be found in the Appendix I and in Bricker and Monismith (2007).

Following separation of the burst-averaged wave and turbulence signals, turbulent stresses were used 


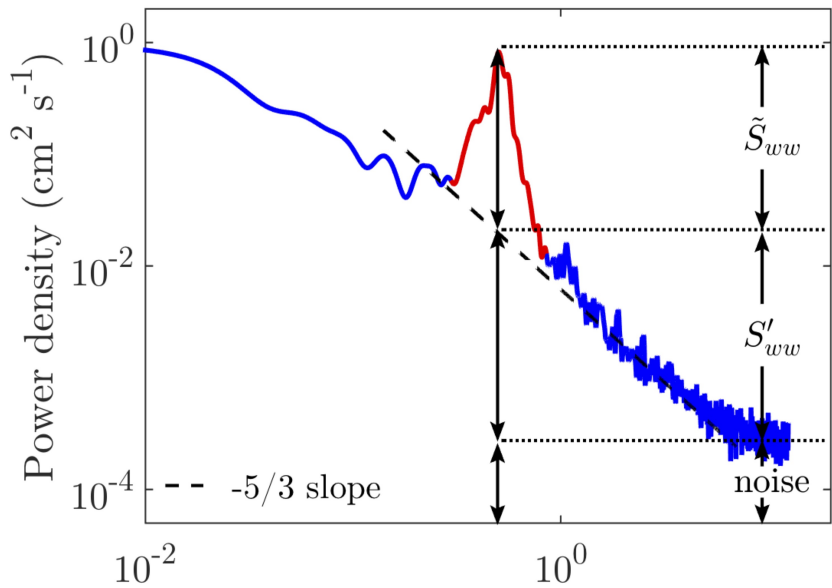

Frequency $(\mathrm{Hz})$

Figure 3.5: Representative power spectral density (PSD) plot of turbulent vertical fluctuations $w^{\prime}$ obtained by ADV for a single measurement bin $\sim 1.5 \mathrm{~cm}$ above the healthy coral. The wave peak $\widetilde{S}_{w w}$, highlighted in red and bound by a $-5 / 3$ fit to the inertial subrange, is subtracted from the total power density (minus noise) to give the turbulent power density $S_{w w}^{\prime}$.

to estimate the components of the TKE budget, given in tensor notation as

$$
\frac{\partial q^{2}}{\partial t}+\bar{u}_{j} \frac{\partial q^{2}}{\partial x_{j}}=\underbrace{-\overline{u_{i}^{\prime} u_{j}^{\prime}} \frac{\partial \bar{u}_{i}}{\partial x_{j}}}_{P s}-\underbrace{\overline{u_{i}^{\prime} u_{j}^{\prime \prime}} \frac{\partial \bar{u}_{i}^{\prime \prime}}{\partial x_{j}}}_{P w}-\underbrace{\frac{\partial \overline{q^{2} u_{j}^{\prime}}}{\partial x_{j}}}_{T t}-\underbrace{\frac{\partial \overline{p^{\prime} u_{j}^{\prime}}}{\partial x_{j}}}_{T p}-\epsilon
$$

where $q^{2}=0.5\left(\overline{u^{\prime} u^{\prime}}+\overline{v^{\prime} v^{\prime}}+\overline{w^{\prime} w^{\prime}}\right)$ is turbulent kinetic energy, $P_{S}$ is turbulence shear production, $P_{W}$ is turbulence wake production (where the double primes signify deviations from a spatial average), $T_{t}$ is advective turbulent transport, and $T_{p}$ is pressure-driven transport [78. Equation 3.2 omits buoyancy production, as well as dispersive and viscous turbulent transport terms, which are all assumed to be negligible. The left-hand side of equation 3.2 represents the temporal change and spatial advection of TKE, respectively, and is equal to zero for a stationary, fully developed shear layer. The degree of development is described by the wave Reynolds number $R e_{o}=\left(\widetilde{u}_{\mathrm{om}} A_{\max }\right) / \nu$, where $\widetilde{u}_{\mathrm{om}}$ is the maximum near-bed wave orbital velocity, $A_{\max }$ is the near-bed wave orbital amplitude, and $\nu$ is fluid viscosity. The dominant terms on the right-hand side of equation 3.2 are production and dissipation $\epsilon$, which are measured directly, and the transport terms are set equal to the difference between production and dissipation. Wake production, which results from vortex shedding from individual canopy elements, is only relevant for algae-covered corals. Turbulence dissipation rates $\epsilon$ were calculated using the inertial subrange dissipation method (IDM), described in Appendix II.

Bed shear stress $\tau_{\text {bed }}$ was calculated using the formulation of Wiberg and Smith (1983) [108], defined 
as

$$
\tau_{\text {bed }}=\sqrt{\tau_{c}^{2}+\tau_{w}^{2}}
$$

where $\tau_{c}$ is bed shear due only to currents and $\tau_{w}$ is bed shear due only to wave orbital motions. The currentinduced bed shear stress is estimated using near-bed TKE outside the wave boundary layer, according to Soulsby and Dyer (1981) 109

$$
\tau_{c}=0.19 \rho q^{2}
$$

The wave-induced contribution is parameterized using the near-bottom wave orbital velocity $\widetilde{u}_{\text {om }}$ and a wave friction factor $f_{w} 110$

$$
\tau_{w}=0.5 f_{w} \rho \widetilde{u}_{\mathrm{om}}
$$

The appropriate wave friction factor is determined by the ratio of the wave amplitude $a$, i.e., half of the wave height, to the bed roughness $k_{b}$, and for the corals studied, is given by $f_{w}=0.4\left(a / k_{b}\right)^{-3 / 4} 111$.

\subsection{Results}

A total of eight ADV records over healthy corals and four ADV records over algae-covered corals met the inclusion criteria for data analysis. Table 3.1 lists key wave and current parameters, including wave

\begin{tabular}{lllllll}
\hline $\begin{array}{l}\text { Coral } \\
\text { case }\end{array}$ & $\begin{array}{l}\text { Root-mean-square } \\
\text { horizontal velocity } \\
u_{\infty, \mathrm{rms}}\left(\mathrm{cm} \mathrm{s}^{-1}\right)\end{array}$ & $\begin{array}{l}\text { Mean current } \\
\text { velocity } \\
\bar{u}_{\infty, c}\left(\mathrm{~cm} \mathrm{~s}^{-1}\right)\end{array}$ & $\begin{array}{l}\text { Maximum wave } \\
\text { orbital velocity } \\
\widetilde{u}_{\text {om }}\left(\mathrm{cm} \mathrm{s}^{-1}\right)\end{array}$ & $\begin{array}{l}\text { Mean wave } \\
\text { period } \\
T(\mathrm{~s})\end{array}$ & $\begin{array}{l}\text { Mean wave } \\
\text { height } \\
H(\mathrm{~cm})\end{array}$ & $\begin{array}{l}\text { Wave Reynolds } \\
\text { number } \\
R e_{o}\end{array}$ \\
\hline \hline H1 & 1.41 & 0.48 & 3.91 & 2.00 & 7.73 & 548.1 \\
$\mathrm{H} 2$ & 1.90 & 0.68 & 4.93 & 2.01 & 9.64 & 873.9 \\
$\mathrm{H} 3$ & 1.96 & 1.57 & 3.04 & 3.72 & 2.02 & 613.8 \\
$\mathrm{H} 4$ & 2.34 & 0.83 & 9.66 & 2.21 & 15.62 & 3695.6 \\
$\mathrm{H} 5$ & 2.35 & 0.28 & 4.80 & 2.28 & 7.42 & 973.9 \\
$\mathrm{H} 6$ & 2.41 & 1.91 & 4.63 & 2.69 & 3.37 & 1030.5 \\
$\mathrm{H} 7$ & 2.54 & 0.37 & 4.99 & 2.10 & 8.87 & 935.0 \\
$\mathrm{H} 8$ & 4.26 & 0.86 & 13.83 & 2.41 & 19.69 & 8229.8 \\
$\mathrm{~A} 1$ & 0.89 & 0.30 & 2.30 & 1.68 & 1.99 & 157.9 \\
$\mathrm{~A} 2$ & 1.08 & 0.41 & 2.78 & 1.27 & 3.87 & 174.7 \\
$\mathrm{~A} 3$ & 1.29 & 0.26 & 2.14 & 1.38 & 2.46 & 112.8 \\
$\mathrm{~A} 4$ & 1.40 & 0.43 & 2.93 & 1.52 & 2.88 & 232.4 \\
\hline
\end{tabular}

Table 3.1: Flow conditions for healthy (H) and algae-covered (A) corals. 
Reynolds numbers, for healthy and algae-covered corals. As conditions varied across records, we chose to normalize velocities and turbulence statistics using the root-mean-squared (rms) velocity, which measures total fluid motion from waves and currents, and is given by

$$
u_{\mathrm{rms}}=\sqrt{\overline{\overline{u^{2}}}}
$$

where the overbar represents time-averaging of the squared instantaneous horizontal velocity $u$ for an individual record. The horizontal free-stream rms velocity $u_{\infty, \mathrm{rms}}$ was calculated for each burst by averaging the ten measurement bins farthest from the coral surface, which varied by less than $3 \%$, indicating a steady, "local" free-stream velocity.

\subsubsection{Velocity Structure and Reynolds Stresses}

Figure 3.6 shows vertical profiles of rms horizontal velocity for healthy coral (in red) and algaecovered coral (in green). Profiles plot the normalized mean \pm one standard deviation of all included bursts ( $n=8$ for healthy corals, $n=4$ for algae-covered corals). RMS horizontal velocities over the healthy corals show a steep increase from the "no-slip" boundary condition at the coral surface and reach the $99 \%$ freestream rms velocity threshold approximately $5 \mathrm{~mm}$ above the coral. For bursts in which rms wave orbital velocities are much larger than mean current velocities, the thicker current-only boundary layer can be
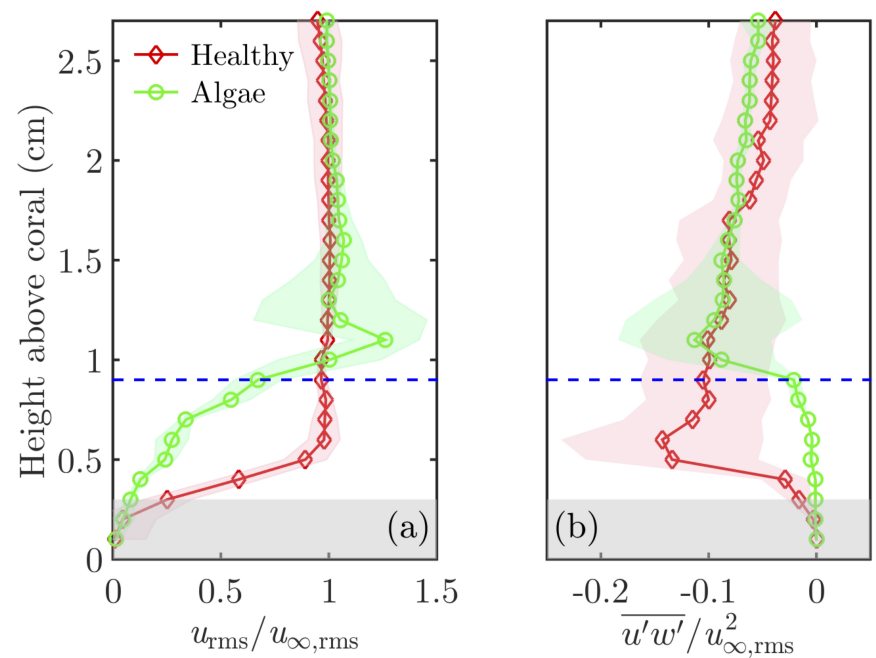

Figure 3.6: (a) Vertical profiles of root-mean-square (rms) horizontal velocity normalized by free-stream rms velocity. Markers indicate heights of measurement bins for ADV, and shaded regions represent \pm one standard deviation from mean. Horizontal blue dashed line indicates approximate height of canopy for algae-covered coral, and gray area represents region of "acoustic reflection" bias, in which data points are less reliable. (b) Vertical profiles of turbulent Reynolds stress normalized by square of the rms free-stream velocity. 


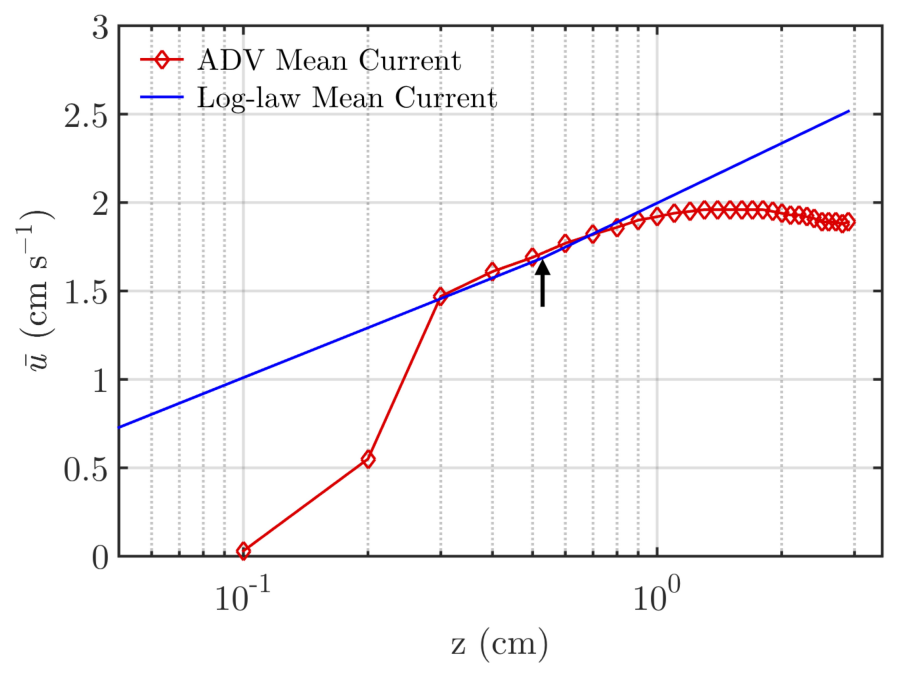

Figure 3.7: Comparison of a theoretical combined wave-current logarithmic profile for mean velocity for an inertial boundary layer (in blue) versus measured mean velocity profile over healthy coral (in red). The arrow indicates the transition from wave to current boundary layer, i.e., where the slope of the logarithmic line increases.

"masked" by the wave velocities in the total rms velocity profile (evident in the log profile below). To determine the degree to which the measured turbulent boundary layer over the healthy corals approximates a theoretical log-law regime, we isolated the mean current profile from a typical ADV burst and compared it to the predicted profile both within the wave boundary layer (WBL) and within the superimposed current boundary layer (CBL). For flows over rough surfaces, the profile of the current is predicted to be logarithmic, with differing slopes for each region, according to

$$
\bar{u}_{c}=\frac{\beta}{\kappa} \ln \frac{30 z}{\gamma}
$$

where $\beta=u_{*, c}^{2} / u_{*, c w}$ and $\gamma=k_{b}$ within the WBL and $\beta=u_{*, c}$ and $\gamma=k_{b c}$ above the WBL 67. $u_{*, c}$ and $u_{*, c w}$ are friction velocities due to steady flow only and combined wave-current flow, respectively. $k_{b}$ is bottom roughness, set equal to the coral polyp size $(\sim 0.25 \mathrm{~cm})$, and $k_{b c}$ is the "apparent" bottom roughness, which includes the actual bottom roughness and an additional roughness component resulting from the drag generated by the WBL and felt by the current boundary layer. The value of $k_{b c}$ is found by matching the mean velocity at the end of the wave boundary layer to the mean velocity at the start of the current boundary layer.

Figure 3.7 shows record $\mathrm{H} 1$ as a representative dataset comparing the profile of the mean current velocity to the theoretical log-law inertial subrange. Excellent agreement between the two profiles exists between approximately $z=3$ and $z=9 \mathrm{~mm}$. The transition from wave boundary layer to current boundary layer-indicated by the increase in slope of the predicted mean velocity profile and marked by the vertical 
arrow - occurs approximately $5 \mathrm{~mm}$ above the coral, matching the thickness estimated from the rms profile.

In contrast to the healthy coral, the profile of rms horizontal velocity over the algae-covered coral (figure 3.6a) is characterized by a distinct inflection point just above the canopy top and a strong shear layer due to the frictional drag imparted on the flow by the algae. Within the canopy, horizontal velocities decay rapidly and exhibit an almost linear profile near the coral surface. Compared to the healthy coral, the algae-covered corals possess reduced velocities near the coral surface, where for example, the $50 \%$ free-stream velocity threshold is reached $8 \mathrm{~mm}$ above the coral surface compared to only $3 \mathrm{~mm}$ above the healthy coral.

Normalized Reynolds stress profiles over the healthy coral (figure $3.6 \mathrm{p}$ ) show a peak approximately $5 \mathrm{~mm}$ above the surface, indicating maximum rates of turbulent mixing very near coral polyps. Above this region, Reynolds stresses remain fairly steady between $z=6$ and $z=9 \mathrm{~mm}$-above the WBL but still within the current boundary layer - suggesting the presence of a constant stress layer typical of equilibrium boundary layer flows. Higher in the water column, turbulent stresses trend toward zero, though $\overline{u^{\prime} w^{\prime}}$ remains non-zero likely due to transported non-local turbulence. The normalized Reynolds stress profile for the algae-covered coral peaks just above the canopy top, where the inflection in velocity occurs. Above this, no constant stress layer is evident; rather, the stresses decrease linearly toward zero. Direct comparison of the profiles shows that Reynolds stresses for the healthy coral do not approach zero until nearly the surface of the coral, whereas the algal canopy dampens Reynolds stresses to close to zero at approximately $8 \mathrm{~mm}$ above the coral-water interface.

\subsubsection{Turbulent Kinetic Energy Budget}

For the healthy coral, vertical profiles of normalized mean TKE (figure 3.8 show a rapid rise in the near-bed region, peaking $\sim 5 \mathrm{~mm}$ above the bed, and remaining constant throughout the upper profile. The algae-covered corals, by contrast, exhibited nearly three times higher levels of TKE above the canopy compared to the healthy corals, owing to the interaction between the flow and the drag generated by the canopy top. Normalized TKE levels for healthy corals demonstrate low variance across wave and tidal conditions; whereas, the algal profile feature higher variability, likely due to the inhomogeneity of algal turfs. Within the canopy, TKE levels are drastically reduced, and fall below healthy coral levels for regions below $z=6 \mathrm{~mm}$.

Turbulence production over the healthy corals, shown in figure $3.8 \mathrm{p}$, exhibits a sharp peak approximately $5 \mathrm{~mm}$ above the coral surface due to the steep velocity gradients and peak Reynolds stresses at this elevation. Above this height, production decreases rapidly and is effectively zero for elevations greater than $z=6 \mathrm{~mm}$. Similarly, turbulence dissipation over the healthy corals (figure $3.8 \mathrm{c}$ ) peaks at $z=5 \mathrm{~mm}$ above the 

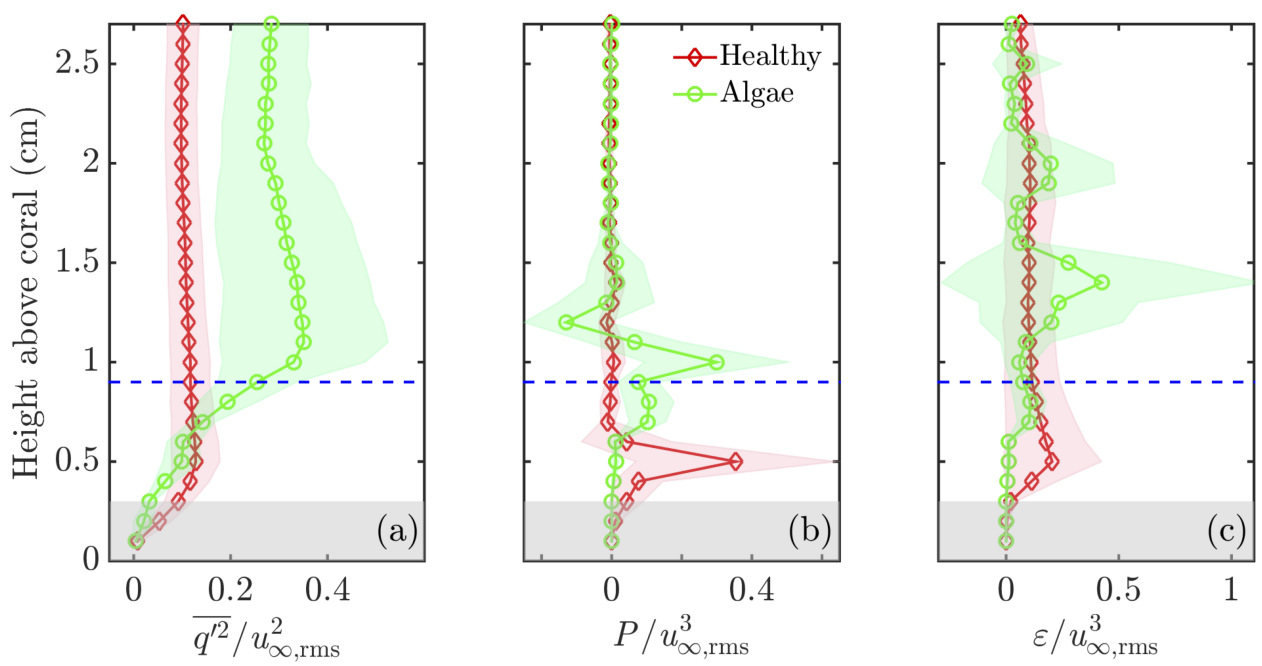

Figure 3.8: Vertical profiles of (a) turbulent kinetic energy (b) turbulent production, and (c) turbulent dissipation rate, normalized by square and cube of the root-mean-square free-stream velocity, respectively. Markers indicate heights of measurement bins for ADV, and shaded regions represent \pm one standard deviation from mean. Horizontal blue dashed line indicates approximate height of canopy for algae-covered coral, and gray area represents region of "acoustic reflection" bias, in which data points are less reliable.

coral surface and is similar in magnitude to production, indicating a local equilibrium balance. The decrease in dissipation rate with height is gradual and remains slightly non-zero throughout the water column, which suggests dissipation of non-local turbulence may be occurring.

Algae-covered corals exhibit two strong peaks in turbulence production above the canopy (3.8p), one positive and one negative, and another moderate production region within the upper canopy, a result of fine-scale wake shedding. The peaks above the canopy result from the velocity shear layer, and the inflection in the velocity profile generates the negative gradients responsible for "negative" production. Higher in the water column, production falls to zero, similar to the healthy corals. The profile of turbulence dissipation over the algae-covered corals (3.8k) shows a peak in $\epsilon$, with large variability, at an elevation higher than the two shear production peaks, indicating nonlocal equilibrium and significant turbulent transport in this region.

\subsubsection{Bed Shear Stress}

For the healthy corals, near-bed values of $q^{2}, \widetilde{u}_{o m}$, and $\overline{u^{\prime} w^{\prime}}$ required to estimate bed shear stress were selected based on their peak values within the boundary layer. However, the algal canopy dampens vertical transport so that peak values of mean and turbulent quantities above the canopy are not appropriate predictors of stresses at the coral surface. For dense canopies, the penetration depth $h_{p}$ of momentum, called the "vertical exchange zone", has been shown to extend to approximately $z / h_{c} \sim 0.7$, where $h_{c}$ is canopy 


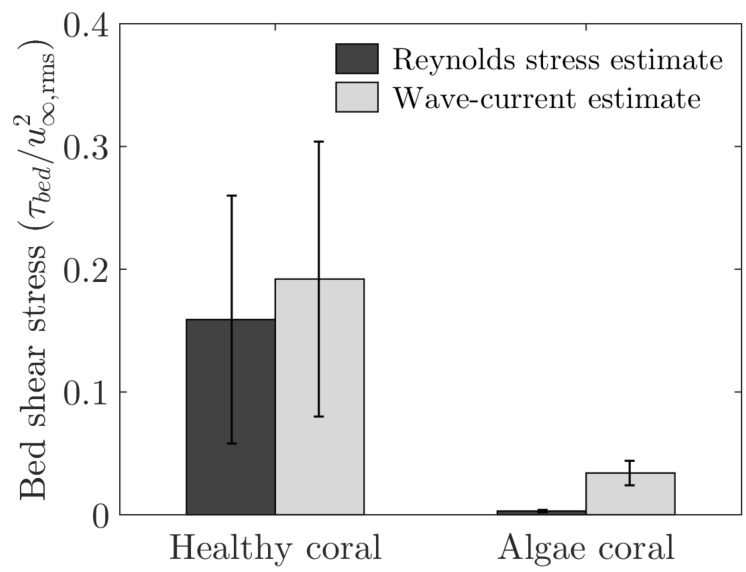

Figure 3.9: Comparison of normalized bed shear stress for healthy and algae-covered corals using two independent techniques and showing statistically significant difference in means between healthy and algae-covered corals (Welch's $t$ test, $t=4.1, d f=7, p<0.01)$. Error bars represent \pm one standard deviation from mean.

height [78. Below this height, turbulent terms remain relatively constant, so values just below $h_{p}$ were used to calculate bed shear stress. These estimates were compared to direct estimates of bed shear stress using total Reynolds stresses $\overline{u^{\prime} w^{\prime}}$, which include contributions from both current and wave motion, according to the relationship $\tau_{\text {bed }}=-\rho \overline{u^{\prime} w^{\prime}}$. Figure 3.9 compares normalized bed shear stress calculated using each technique for healthy and algae-covered corals. Results show that the mean bed shear stress for healthy corals was nearly an order of magnitude greater than for the algae-covered corals (Welch's $t$ test, $t=4.1$, $d f=7, p<0.01)$.

\subsubsection{PIV Spatial Analysis of Mean and Turbulent Flow Structure}

Figure 3.10 a shows the rms velocity field for PIV data consisting of 900 image pairs, totaling $30 \mathrm{~s}$. The mean of rms velocities for the top $1 \mathrm{~cm}$ is $0.85 \mathrm{~cm} \mathrm{~s}^{-1}$, and the vertical profiles show a trend similar to the ADV measurements with a sharp decrease near the surface. A key difference, however, is the spatial variability of the velocity around the coral surface. The leading edge of the coral structure (left side of image) causes a much smaller decrease in near-surface velocities compared to the flatter coral top (right side of image), which is attributed primarily to accelerating flow at the leading edge of the spherical bluff body. Normalized Reynolds stresses $\overline{u^{\prime} w^{\prime}}$ (figure $3.10 \mathrm{~b}$ ) exhibit a similar vertical profile compared to ADV measurements with low stresses near the surface, a steep rise to peak values within $1 \mathrm{~cm}$ of the coral, and a gradual decrease for regions above. The peak Reynolds stress values for the right half of the imaging window $(\sim 0.15-0.25)$ correspond well to ADV values, while the left half of the imaging window shows stress values nearly $50 \%$ higher and a much sharper gradient near the coral surface. The spatial variation in stresses agrees with similar spatial variations in velocity and suggests large-scale topography plays a significant role 

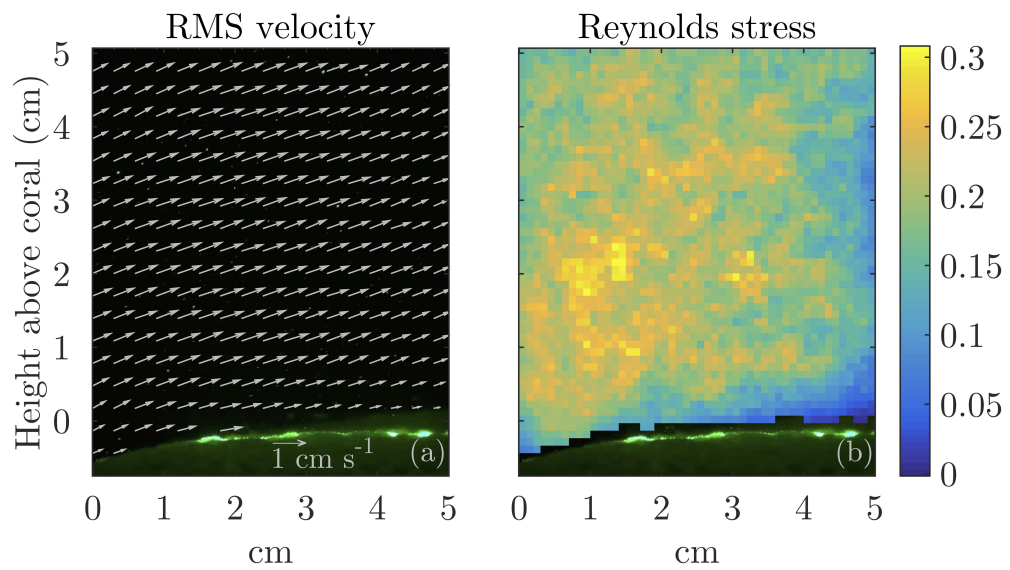

Figure 3.10: (a) Vector field of normalized root-mean-square (rms) velocities over a healthy coral from particle image velocimetry. For clarity, only every third vector is plotted. (b) Plot of turbulent Reynolds stress $\overline{u^{\prime} w^{\prime}}$ normalized by square of rms free-stream velocity.

in creating spatial heterogeneity in the flow.

Normalized TKE (figure 3.11 a $)$ indicates a relatively constant value $(\sim 0.7-0.8)$ for elevations above $0.5 \mathrm{~cm}$, which compares well to the TKE vertical profile seen in ADV measurements. Magnitudes of TKE, however, are higher for PIV data, likely owing to low particle seeding, which can create larger errors in instantaneous velocity estimates, thereby biasing terms in the TKE budget. Turbulence production (figure 3.11p) shows large peaks in the lowest $1 \mathrm{~cm}$ of the boundary layer and nearly zero production above this height, similar to the ADV profile, while turbulence dissipation (figure 3.11) indicates low levels close to the surface and patchy increased rates higher in the water column. Peak normalized values for both production and dissipation are comparable to ADV estimates.
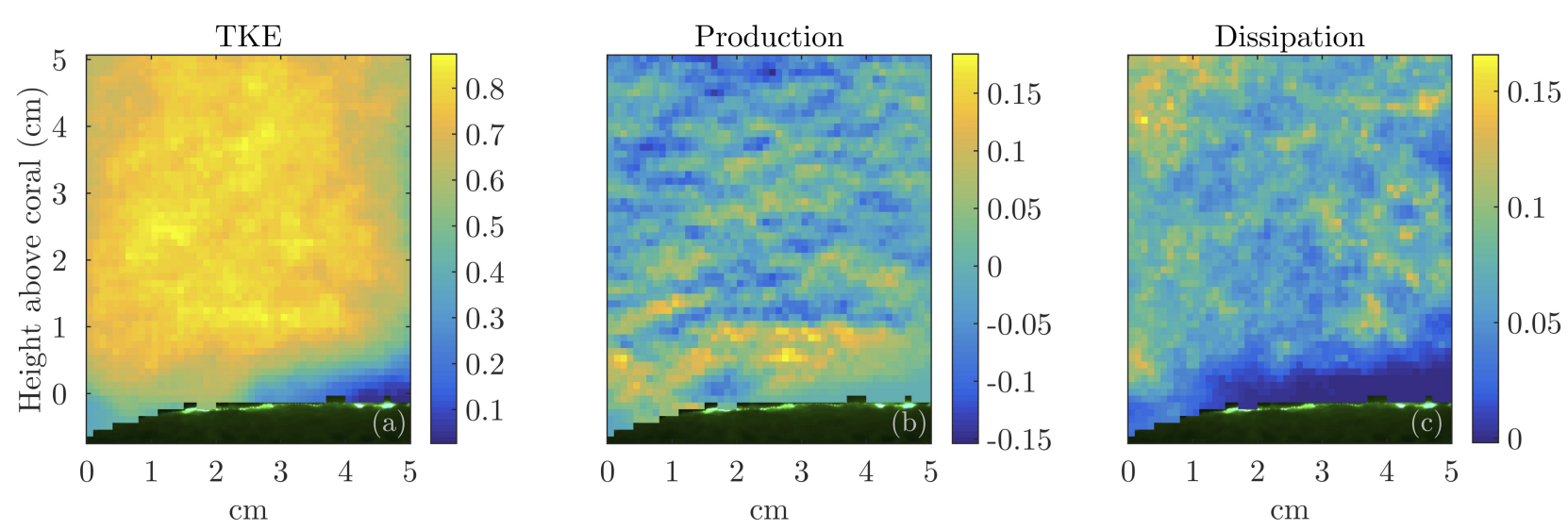

Figure 3.11: (a) Plot of turbulent kinetic energy over healthy coral normalized by square of root-mean-square (rms) freestream velocity. Plots of turbulence production (b) and dissipation (c) normalized by cube of rms free-stream velocity. 


\subsection{Discussion}

Reef productivity and metabolism are driven both temporally and spatially by nutrient uptake rates, which have been shown to operate near the limits of mass transfer in reef communities $[22$. Under conditions of mass transfer limitation, nutrient uptake rates are physically limited by the transport of nutrients across concentration boundary layers, rather than biologically limited by rates of cellular transport and assimilation. This indicates that reef primary producers such as coral or algae take up nutrients as fast as physically possible, and thus, in the long term, flow-mediated enhancement of mass transfer can augment rates of benthic primary production. Predicting hydrodynamic control of nutrient mass transfer rates requires an adequate description of the near-bed turbulence that is mechanistically correct at the scale of the inertial and concentration boundary layers $(<1 \mathrm{~mm})$. Our results indicate that two distinct fine-scale turbulent boundary layer regimes exist over healthy corals and algae-covered corals and that the transition from a wall-bounded inertial layer over healthy corals to a plane-mixing layer regime over algae-covered corals has significant implications for mass transfer to corals.

\subsubsection{Boundary Layer Characterization for Healthy Corals}

Results describing mean and turbulence profiles over the healthy corals (figures 3.6 and 3.8 suggest that key features of a canonical wall-bounded shear layer driven by combined wave-current flow are present. First, fully developed turbulent boundary layers possess an inertial sublayer defined by a logarithmic velocity profile (the "law of the wall") extending from $30<z^{+}<300$, where $z^{+}=u_{*} z / \nu$ is the dimensionless wall unit normalized by the viscous scale 61 . The agreement between the predicted and measured mean velocity profiles illustrated in figure 3.7 supports the existence of an inertial sublayer over the healthy corals. However, the plotted inertial subrange extends only to $z^{+} \sim 60$, indicating transitional, rather than fully developed, turbulence (confirmed by the sub-critical wave Reynolds number). More energetic wave conditions-e.g., faster wave orbital velocities or larger wave orbital amplitudes-would enhance turbulence development, extending the sublayer vertical range and enhancing near-surface mixing.

A second feature of wall-bounded shear layers is the local balance of turbulence production and dissipationevident in the TKE budget profiles in figure 3.8 . Eddy production provides a main mechanism for advective fluid exchange between the coral surface and water column, driving scalar mixing and mass flux. To describe the length scale over which an over-turning eddy mixes and the rate at which it advects, we estimated the turbulent mixing length $\ell_{m}$ and eddy viscosity $\nu_{e}$, respectively, which scale linearly with distance from the wall according to $\ell_{m}=\kappa z$ and $\nu_{e}=u_{*} \kappa z \mid 61$. Direct estimates of $\ell_{m}=\left|\overline{u^{\prime} w^{\prime}}\right|^{1 / 2} /\left|\partial u_{\mathrm{rms}} / \partial z\right|$ and $\nu_{e}=-\overline{u^{\prime} w^{\prime}} /\left(\partial u_{\mathrm{rms}} / \partial z\right)$ at approximately $4 \mathrm{~mm}$ above the coral surface give mean values of $\ell_{m}=0.16 \pm 0.1 \mathrm{~cm}$ 
and $\nu_{e}=0.64 \pm 0.4 \mathrm{~cm}^{2} \mathrm{~s}^{-1}$, which closely match predicted values. Taken together, the agreement between experimental and predicted values for mean velocity, mixing length, and eddy viscosity, as well as the local balance in TKE components, strongly suggests that the turbulent boundary layer structure over the healthy corals is a canonical wall-bounded turbulent shear layer, and traditional log-layer dynamics can be used to parameterize predictions of flow and scalar flux.

Though ADV measurements taken on the flat section of the coral top suggest a wall-bounded shear layer, PIV results indicate significant spatial heterogeneity in mean and turbulent flow dynamics exists across the varied topography of an individual coral. When describing the fine-scale boundary layer within centimeters of the coral surface, point measurements such as those recorded by an ADV may demonstrate only limited predictive value for considerations of integrated coral mass flux. Rather, estimates need to account for the effects of local topography and large-scale geometry on boundary layer dynamics, especially given that such variability influences coral morphological development and plasticity [14.

\subsubsection{Boundary Layer Characterization for Algae-covered Corals}

Mean and turbulence profiles over algae-covered corals illustrate a much different boundary layer structure, more closely approximating a plane mixing layer. Similar to unconfined canopy flows in terrestrial systems, the active turbulence at a canopy top forms when two co-flowing streams with different velocities interact along a midplane to produce a turbulent shear layer via propagation of Kelvin-Helmholtz instabilities [79]. Several key hydrodynamic features characterize the plane mixing analogy for canopy turbulence. First, profiles of mean velocity and turbulent statistics exhibit strong vertical inhomogeneity, with a characteristic inflection point in velocity at the canopy top, and rapid decay of turbulent terms within the canopy - results evident in the rms velocity and TKE budget profiles. Qualitatively, the abrupt dampening of these terms within the canopy top is similar to many previously reported "dense" canopy flow profiles 83].

The velocity inflection identifies the location of maximum shear and determines the canopy shear length scale according to $\ell_{S}=u_{\mathrm{rms}}\left(h_{c}\right) / u_{\mathrm{rms}}^{\prime}\left(h_{c}\right)$, where $h_{c}$ is the canopy height and the prime indicates a spatial derivative. $\ell_{S}$ controls the size of active turbulence above and within the canopy top [83], and for the algae-covered corals, the mean shear length scale measures $\ell_{S}=0.29 \pm 0.01 \mathrm{~cm}$, which compares well with reported values of $0.27-0.72 \mathrm{~cm}$ for a canopy of equivalent height, where smaller values correlate with denser canopies 78 . Though this turbulent length scale is larger than the turbulent mixing length over the healthy coral, those eddies occur directly adjacent to the coral surface, whereas eddies induced by plane mixing occur at the canopy top and only have an overturning length $\sim 30 \%$ of the canopy height. This indicates that momentum (and mass) is not actively exchanged with the lower parts of the canopy or the coral surface. 
The plane mixing layer analogy for canopy flow suggests a significantly altered TKE budget profile, described by a local imbalance between production and dissipation in the "roughness sublayer", i.e., the region just above the canopy $\left(h_{c}<z<2 h_{c}\right)$ 83. Production and dissipation profiles in figure 3.8 highlight this local imbalance, suggesting non-equilibrium turbulence in the roughness sublayer with significant pressure and turbulent transport vertically away from the coral surface. Finally, efficiency of momentum transfer can be measured by the Reynolds stress correlation coefficient, $r_{u w}=\overline{u^{\prime} w^{\prime}} /\left(\sigma_{u} \sigma_{w}\right)$, where $\sigma_{u}$ and $\sigma_{w}$ are standard deviations of horizontal and vertical velocity fluctuations, respectively. Typical values of $r_{u w}$ for a plane mixing layer approach -0.5 , compared to -0.32 for an inertial layer, an indication of more effective momentum transfer 83. Just above the canopy top, our results estimate $r_{u w}=-0.59 \pm 0.08$, matching predicted levels well and suggesting high momentum (and scalar) transfer in this region. Though the algal canopy enhances turbulent mixing and fluid exchange between the overlying water column and canopy top, its presence and density serve to buffer the coral surface from these exchanges and prevents the coral from benefitting from greater mixing rates.

\subsubsection{Implications for Biology}

Many biological processes vital to coral health depend on the mean and turbulence flow structure near the coral surface, which control the diffusive boundary layer thickness and affect the mass flux "bottleneck". Rates of mass flux $m$ at the coral-water interface can be described by mass-transfer theory according to $m=S\left(C_{b}-C_{s}\right)$, where $C_{b}$ and $C_{s}$ are the bulk fluid and surface nutrient concentrations, respectively, and $S$ is a mass transfer coefficient dependent on molecular diffusivity and proportional to $\tau_{\text {bed }}^{1 / 2} 49$. This scaling suggests that for a fixed concentration gradient at the coral surface, the nearly nine times higher bed shear stress reported for healthy corals compared to algae-covered corals results in three times greater rates of mass transfer under the same ambient flow conditions. Though velocities reported here are relatively slow due to sheltering of the field site, faster flows should exhibit similar effects as higher velocities would enhance bed shear stresses for both regimes; however, relative enhancements in mass flux would depend on the specific local conditions, e.g., surface roughness and canopy density.

Bed stress values alone, however, do not fully describe the hydrodynamic impact of the algal canopy. RMS velocity profiles between the two regimes, for example, demonstrate that the algal canopy dampens flow near the coral surface, which allows the DBL thickness to increase and reduces the efficacy of diffusive transport. Additionally, algal canopies actively photosynthesize, removing $\mathrm{CO}_{2}$ before it reaches the coral surface (for use by zooxanthellae) and releasing $\mathrm{O}_{2}$ adjacent to the surface, strengthening gradients in the DBLs of each species. More efficient mixing at the canopy top may produce a positive feedback loop, 
promoting algal primary productivity and growth while reducing a coral's physical access to nutrients.

Within a broader ecological context, these results may be important in the framework of shifting benthic community composition and a coral's ability to recover from large-scale natural or anthropogenic disturbances. Seasonal-scale events, like hurricanes or disease outbreaks, and decadal-scale events, such as ocean warming and acidification, can physically damage reef structures and stress corals into bleaching events, rendering them vulnerable to invasion of algal competitors [11. Coral resilience depends on the ability to re-recruit expelled zooxanthellae, to efficiently exchange nutrients, and to mitigate thermal stress via advective transport. Turbulent hydrodynamics are key drivers of these processes. Our results demonstrate that the presence of an algal canopy on a coral surface shifts the turbulent boundary layer regime from a wall-bounded inertial layer to a plane mixing layer, and the hydrodynamic consequences of this transition lead to significant reductions in mass transfer, negatively impacting the ability of a coral to obtain resources and limiting its metabolic productivity. 


\section{Chapter 4}

\section{Experimental Investigation of}

\section{Unidirectional Flow Past Smooth and}

\section{Rough Hemispheres}

This chapter describes a set of laboratory measurements made over hemispheres with varying surface roughness, designed to represent idealized models of various massive coral species. The objective of this experimental investigation is to quantify how the addition of different bar-type roughness elements to a hemispheric bluff body exposed to steady, unidirectional flow affects: 1) near-surface flow structure and turbulence characteristics, and 2) downstream wake formation and mixing dynamics. Implications of the resulting hydrodynamics on biophysical controls of coral health will be discussed. The chapter is organized as follows: first, a brief review of previous work on flow past spheres and hemispheres will be discussed, along with a presentation of the specific experimental questions addressed by this work. Next, fabrication techniques for model hemispheres, laboratory facilities, and experimental measurement apparatus will be described, followed by a section presenting the data analysis techniques for both mean and turbulent quantities. Experimental results comparing model morphologies and Reynolds number regimes will be presented in two separate sections, first, for mean quantities, and second, for turbulence statistics. Finally, the chapter closes with a discussion offering interpretations of the presented results in light of previous findings and with regard to their implications for thermal and scalar transport and associated biological impacts. 


\subsection{Experimental Questions}

Descriptions of flow past a sphere have a long history in the physics and engineering literature. Seminal contributions came in the mid-nineteenth century from Irish mathematician Sir George Stokes, who derived an analytical solution for steady, low Reynolds number flow (i.e., $R e<<1$ ) around a sphere - a regime known as creeping or Stokes flow 61. Such low Re flow regimes, however, are essentially never present in a coral's habitat. Rather, the most typical hydrodynamic regimes in a reef system are moderate to occasionally high Reynolds number flows. In contrast to low $R e$ flow, characterizations of high $R e$ flow past a sphere do not readily admit an analytical solution, and thus early studies relied on empirical measurements.

Achenbach (1972) directly measured the total drag on a smooth sphere and found evidence for a critical Reynolds number between $3-4 \times 10^{5}$. Below $R e_{\text {crit }}$, the drag coefficient $c_{D}$ is $\sim 0.5$ and the boundary layer separation angle $\phi$ is $\sim 82^{\circ}$, while for $R e>R e_{c r i t}, c_{D}$ drops sharply to $0.1-0.2$, and $\phi$ rises to $115-120^{\circ}$ [12]. Taneda (1978) used flow visualizations of wake coherent motions to support Achenbach's $R e_{c r i t}$, finding a transition from "progressive wave motion" to pairs of streamwise line vortices at approximately $R e=3.8 \times 10^{5} 113$. In an additional set of experiments with roughened spheres, Achenbach also demonstrated a dependence of $R e_{\text {crit }}$ on the size of the surface roughness, such that increasing roughness element size (as a ratio of sphere diameter) decreases the critical Reynolds number, accelerating the transition to a turbulent wake, yet also producing a larger $c_{D}$ at high $R e$ compared to smooth spheres 85 .

More recent work investigating flow past spheres has employed computationally expensive direct numerical simulations (DNS), which solve the Navier-Stokes equations directly. Due to computational requirements, simulations have typically been limited to Reynolds numbers up to 1000 and only for smooth surfaces. Johnson and Patel (1999) reported stable, separated, axisymmetric toroidal vortices for $R e<210$, above which pressure gradients overtake stabilizing viscous forces and axial symmetry breaks down, producing streamwise vortices with reflection symmetry [114]. Tomboulides and Orszag (2000) extended simulations to $R e=1000$, finding a second transition to time-dependent vortex shedding between $350<R e<450$, and a loss of planar symmetry with chaotic vortex orientation for $R e=500$, above which Kelvin-Helmholtz instabilities create a turbulent wake while still maintaining a large-scale structure [115].

Using investigations of flow past a sphere to draw conclusions on the dynamics of flow over hemispherical corals is only informative to first order. The presence of a solid boundary surrounding a coral bluff body, i.e., the ocean floor, affects the formation and structure of the downstream wake relative to a freely exposed sphere. Additionally, the incident flow is not vertically uniform, but rather represents a wall-bounded shear layer (and nearly always turbulent in the natural environment), which also impacts the distribution of velocity and turbulence dynamics. For shear flow past a sphere, the critical Reynolds number is lower than 
for a comparable uniform flow, and $R e_{\text {crit }}$ decreases as the strength of the shear increases 116. Similarly, for shear flow past a smooth, wall-mounted hemisphere, the drag coefficient has been shown to be a function of fluid viscosity, friction velocity (i.e., a measure of shear strength), and hemisphere diameter [117. Thus, for corals embedded in a sheared bottom boundary layer, the polar separation angle and transition of wake characteristics occurs at lower velocities than for a free-stream sphere.

Parameters typically of interest in the engineering literature, such as separation angle and drag coefficient, also have biological implications on the health of corals. A delayed boundary layer separation, for example, implies that a greater percentage of the coral surface area is in contact with high velocity, highly sheared flow, and thus, more efficient at interfacial mass flux. Additionally, for bluff bodies like corals in which pressure drag dominates, a larger drag coefficient indicates greater eddy formation in the downstream wake, which promotes dispersion of released chemicals or larvae. However, little is known about the distribution of turbulent kinetic energy budget components over hemispheres or levels of surface shear, which directly impact an individual coral's rate of mass transfer and health. Furthermore, the effect of adding bar-type roughnessas opposed to roughness height to sphere diameter ratios as employed by Achenbach — on boundary layer hydrodynamics is not well understood, an especially important question given the similarity of the surface morphology of certain species of brain coral to engineered bar roughness. This gap in understanding leads to the specific research questions addressed in this chapter:

1. What is the effect of d-type and k-type roughness on the boundary layer hydrodynamics and downstream wake structure of a wall-bound hemisphere?

As highlighted above and in previous chapters, a significant body of literature exists describing flow past rough hemispheres and flow over various 2-dimensional bar roughnesses on a flat plate. However, no prior work has investigated the hydrodynamic impact of d- and k-type roughness elements positioned along a highly curved surface - designed to represent an idealized coral structure. It is expected that by altering the angle of incidence between the incoming flow and the curved roughness features, a significantly different hydrodynamic regime will be produced compared to either flat-plate roughness or a traditionally surfaceroughened sphere. In particular, the near-surface turbulence structure and downstream wake dynamics will be investigated as these parameters have significant influence on interfacial mass flux and far-field mixing, respectively.

2. What are the implications for mass transfer, i.e., coral health, resulting from the altered hydrodynamics due to varying surface roughness?

Descriptions of purely hydrodynamic results are useful and important in their own right and may be applied across a range of potential physical systems, such as roughened heat exchangers or airfoil riblets. 


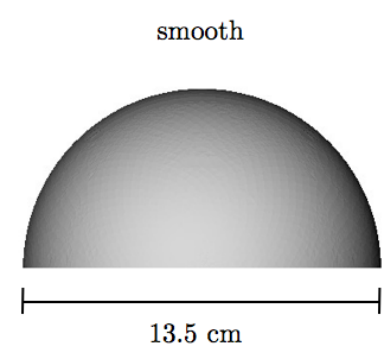

(a)

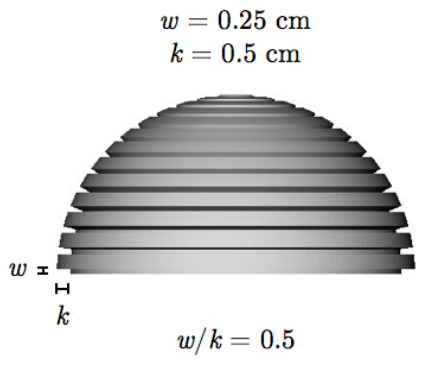

(b)

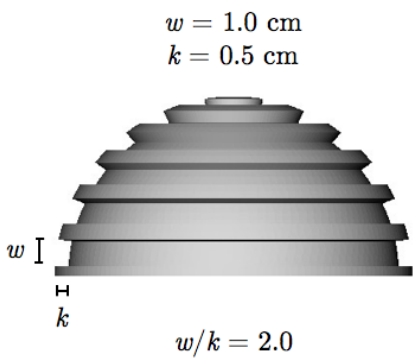

$(c)$

Figure 4.1: CAD models of idealized coral geometries. (a) Smooth hemisphere. (b) D-type roughness with $w / k$ ratio equal to 0.5 . (c) K-type roughness with $w / k$ ratio equal to 2.0 .

However, given the overarching theme of this dissertation, it is critical to apply these physical processes to questions affecting coral health and sustainability, in particular, relative rates of near-surface mixing, coral-water column mass exchange, and far-field scalar transport. Scaling arguments will be applied to estimate potential rates of nutrient uptake. The impact of hydrodynamics on critical biophysical process, e.g., calcification, particle filtration, larvae dispersal, recovery from disturbances, etc., will be discussed.

\subsection{Experimental Methods}

\subsubsection{Model Coral Geometries}

In order to determine the effect of surface roughness on boundary layer and wake hydrodynamics, four coral models of varying roughness type were designed and fabricated. Three of the four models were idealized designs of massive corals representing: 1) a hemisphere with a perfectly smooth surface, 2) a hemisphere with d-type roughness $(w / k=0.5)$, and 3$)$ a hemisphere with k-type roughness $(w / k=2)$. The idealized coral models were designed using FreeCAD, an open-source parametric 3D computer-aided design modeler, and are shown in Figure 4.1. Roughness elements were implemented as ridges of concentric circles with a height of $0.5 \mathrm{~cm}$ and ridge spacing of $0.25 \mathrm{~cm}$ and $1 \mathrm{~cm}$, respectively, and the total model diameter, inclusive of ridge height, was $13.5 \mathrm{~cm}$ for each model. Strictly defined, the intended d-type and k-type roughness spacing only exists on line integrals passing through the midplane of the hemisphere; however, this design simplification was necessary to isolate the effect of roughness type, and all measurements were collected along the model midplane. The radial symmetry of the design eliminated any dependence of coral orientation with respect to the incident flow field.

In addition to the three idealized coral models, a fourth model created from an actual coral skeleton was fabricated. With the assistance of colleagues Prof. Dr. Christian Laforsch and Robert Sigl at the Univer- 

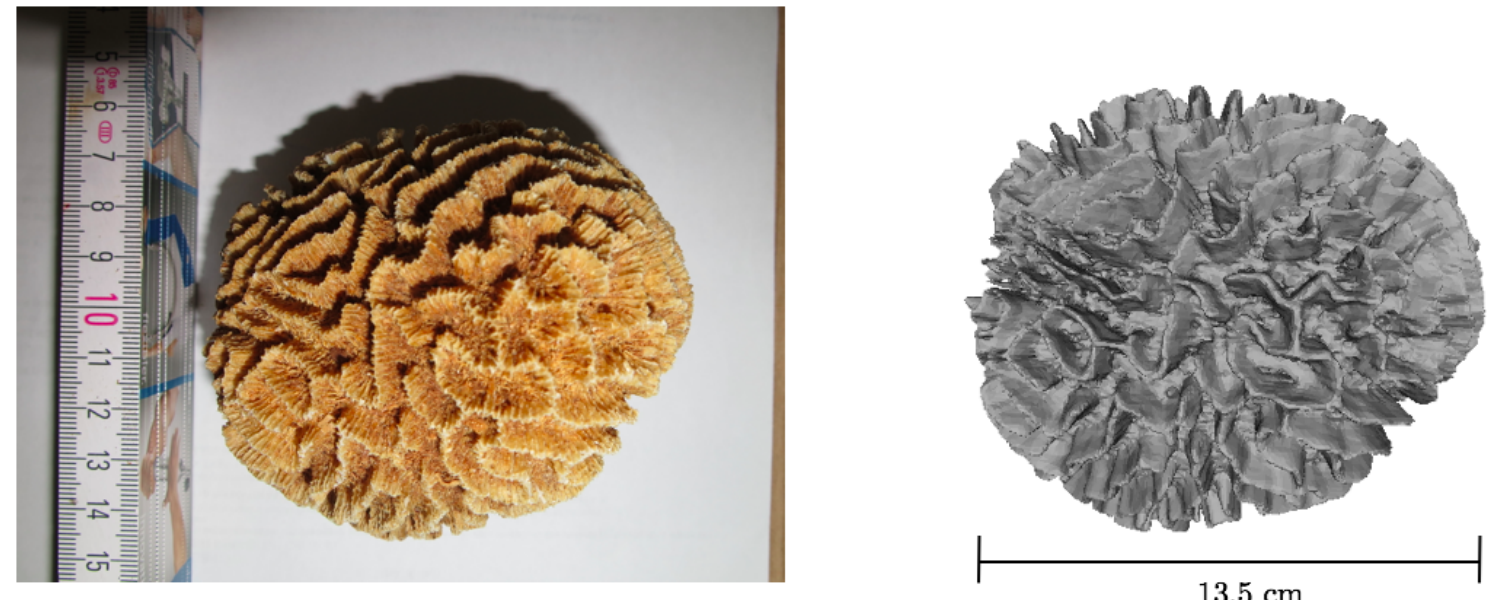

Figure 4.2: Calcium carbonate skeleton of Platygyra coral (left) and resulting x-ray computed tomography scan (right). The computer model of the skeleton has been scaled up to an approximate diameter of $13.5 \mathrm{~cm}$ to match the size of the idealized models.

sität Bayreuth in Germany, a calcium carbonate skeletal structure from a brain coral was obtained courtesy of the Zoologische Staatssammlung München (The Bavarian State Collection of Zoology). The skeleton sample was collected in the Indian Ocean off the coast of Mombasa, Kenya, and classified as genus Platygyra, though identification at the species level was not available. Prof. Dr. Laforsch and Mr. Sigl performed a high-resolution x-ray computed tomography $(\mathrm{CT})$ scan using a Siemens ${ }^{\circledR}$ Somatom ${ }^{\circledR}$ Emotion 16 CT scanner at a tube voltage of $110 \mathrm{kV}$ (Eff mAs 64) and spatial resolution of $0.46 \times 0.46 \times 0.5 \mathrm{~mm}$ (size of voxel, i.e., volume element) with a slice thickness of $0.75 \mathrm{~mm}$. The scan was performed at the private radiology institute Radiologie im Duererhof (Bayreuth, Germany), and the data was acquired using the integrated Somaris software (Syngo CT 2007, Siemens Healthcare GmbH). Post-processing and 3D reconstruction of the DICOM format files was performed using the software package AMIRA 5.5 (Mercury Computer Systems, Inc., France) using an isosurface threshold of -190 Hounsfield Units (standard computed tomography units) to identify coral surfaces, a technique developed by Laforsch and colleagues [118]. The final output produced a stereolithography (.stl) file used to create the fused deposition models (and for use in future numerical simulations). Figure 4.2 shows a photograph of the coral skeleton and a corresponding image of the resulting CT model.

The digital CAD files of the three idealized models and one skeleton model were used as templates to create physical models of the corals through fused deposition modeling. Due to the simplified geometry of the idealized models, 3D printing of these models was able to be performed using the MakerBot ${ }^{\circledR}$ Replicator 2 Desktop 3D printer at the University of Virginia Scholar's Lab in Alderman Library. Models were printed 


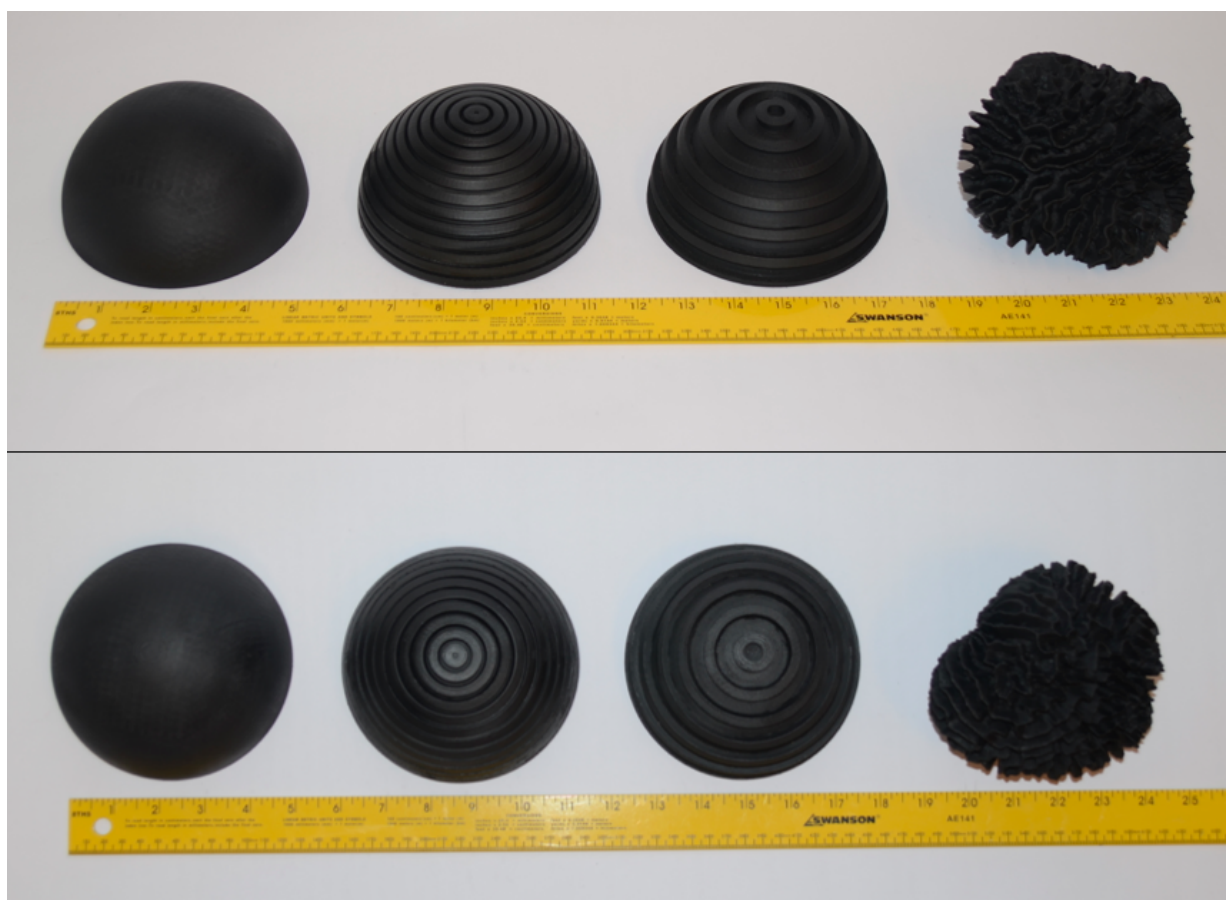

Figure 4.3: Side-angle photograph of the 3D-printed and spray painted coral models (top). Top-down photograph of the coral models (bottom). From left: Smooth hemisphere; hemisphere with $w / k=0.5$; hemisphere with $w / k=2.0$; and CT-scanned coral skeleton.

using polylactic acid (PLA) filament with a layer height of $0.2 \mathrm{~mm}$ and $50 \%$ infill. Upon completion, each idealized model was lightly sanded to remove the small ridges from filament layering and spray painted matte black to reduce reflections of the laser light used during PIV data collection. The resolution of the skeleton model was too detailed for the capabilities of the Replicator 2 printer, which instead required that the skeleton be printed at the University of Virginia Mechanical Engineering Department's Rapid Prototyping Lab (RPL). The skeleton model was printed with a Stratasys ${ }^{\circledR}$ uPrint SE Plus printer using acrylonitrile butadiene styrene (ABS) thermoplastic at a resolution of $0.25 \mathrm{~mm}$ and utilizing water-soluble support material. As with the idealized models, the completed skeleton model was spray painted matte black. Figure 4.3 shows the final 3D printed models in order of increasing topographic complexity.

\subsubsection{Water Tunnel Facilities and Model Setup}

Experimental measurements of flow over the coral models were collected using the UVA Mechanical Engineering Department's recirculating water tunnel. The Rolling Hills Research Corporation Eidetics ${ }^{\circledR}$ Flow Visualization Water Tunnel Model $1520 \mathrm{HK}$ is a closed circuit, continuous flow channel with a tempered glass test section measuring 60 inches long by 20 inches high by 15 inches wide, and permits visual access from the top, sides, bottom, and axially (via a downstream window) for video recording and laser instrumentation. 


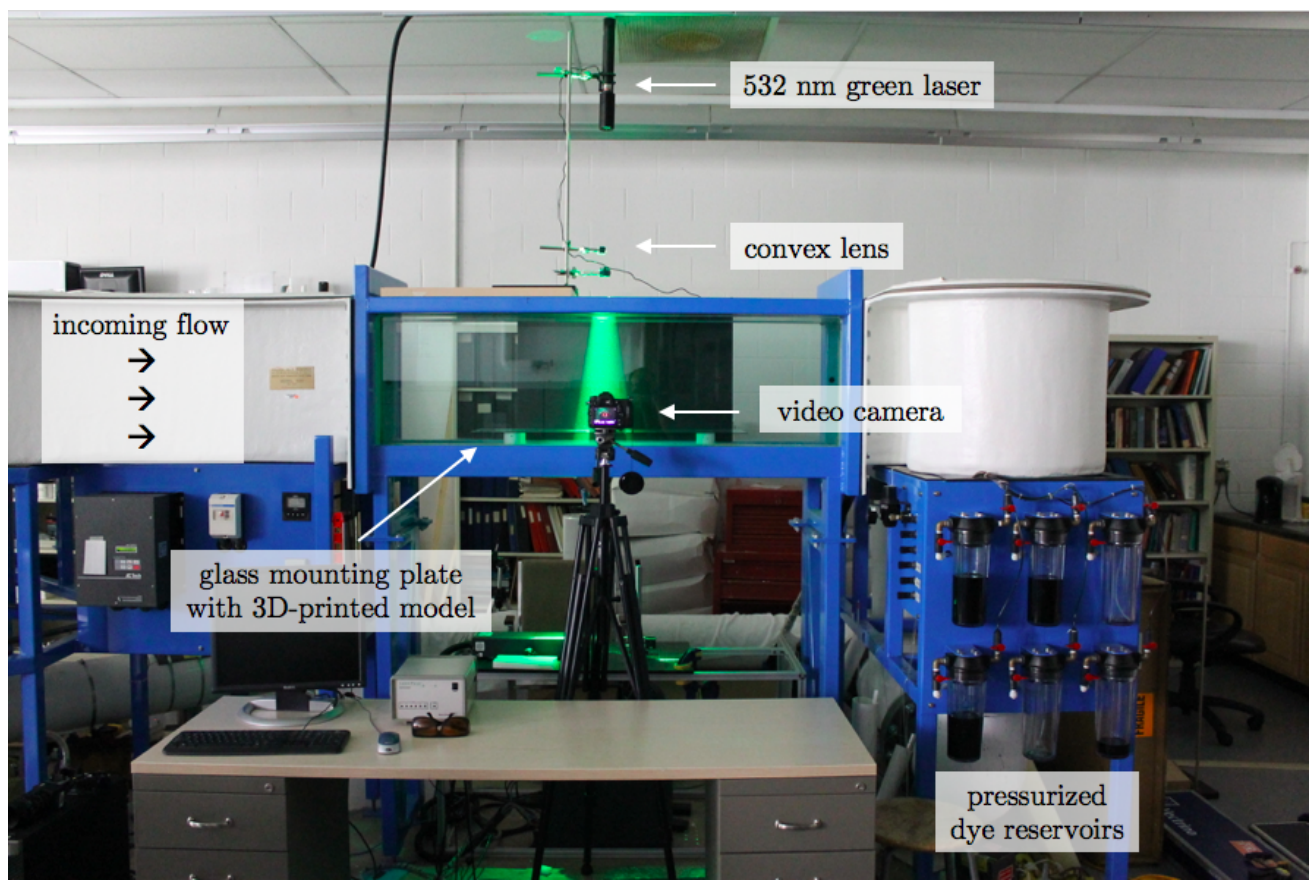

Figure 4.4: Photograph of Rolling Hills 1520 recirculating water tunnel with 3D-printed coral model affixed to a glass mounting plate and experimental particle image velocimetry setup.

The water tunnel has a capacity of 1,000 gallons, a velocity range from 0 to $30.5 \mathrm{~cm} \mathrm{~s}^{-1}$, and an incident rootmean-square (RMS) turbulence intensity of $<1.0 \%$, achieved through a series of upstream flow straighteners. Flow is driven by a 7.5 horsepower centrifugal pump, and the water tunnel is also outfitted with a pressurized 6-color dye system for flow visualization. Schematics for the design and layout of the water tunnel are publicly available at the Rolling Hills Research website (www.rollinghillsresearch.com). With the assistance of fellow MAE graduate student William Eberhardt, I assembled the entire water tunnel system in the summer of 2009.

To standardize the placement of models within the water tunnel test section during data collection, each model was mounted on a separate sheet of plate glass measuring 36 inches long by 14.75 inches wide by $3 / 32$ of an inch thick. Each plastic model was affixed to the center of a glass plate using a waterproof all-purpose adhesive (Welder ${ }^{\mathrm{TM}}$ Adhesive, Homax Products, Inc.) and allowed to set for 24 hours. The glass test section of the water tunnel is framed by powder-coated steel supports, which block horizontal visual access to the bottom of the test section. This required elevating the glass mounting plates above the test section frame, which was achieved by affixing four 2-inch long PVC tubes with a diameter of 1.25 inches at each corner of the underside of the mounting plates. To prevent the models and mounting plates from moving during testing (due to the drag force imparted on the model by the flow), a separate sheet of heavy plate glass measuring XX inches wide by $\mathrm{YY}$ inches long by ZZ inches thick was positioned in the middle 
of the test section and held in place by suction cups attached to the bottom of the glass test section. Four additional 1/2 inch high PVC tubes with a diameter of just under 1.25 inches were affixed to the corners of the base plate and served as locking feet for the model-mounted glass plates. Figures 4.4 and 4.6 show a centrally positioned glass-mounting plate and attached PVC feet mechanism within the water tunnel test section.

\subsubsection{Particle Image Velocimetry}

Measurements of flow over the coral models were obtained using a custom-built particle image velocimetry (PIV) experimental setup. A $325 \mathrm{~mW}$ diode-pumped solid-state laser (Laserglow Technologies ${ }^{\circledR}$ Hercules Series) was positioned centrally on a wooden platform clamped to the top of the water tunnel test section. The continuous wave laser produced a $532 \mathrm{~nm}$ wavelength green light, which was directed vertically downward through a pair of convex lenses to produce a 2-dimensional laser sheet measuring approximately $10 \mathrm{~cm}$ by $10 \mathrm{~cm}$. Unlike a typical commercial PIV system which pulses the laser light and synchronizes the image capture, the laser in this system is constantly on and the image capture occurs continuously. Perpendicular to the laser sheet and level with the coral models, a digital single-lens reflex (DSLR) camera (Nikon Corporation ${ }^{\circledR}$ D7000) with a $24-85 \mathrm{~mm}$ macro lens was mounted on a tripod (Smith-Victor Corporation ${ }^{\circledR}$ ). Figure 4.4 illustrates the experimental PIV setup. The DSLR camera was set to record in video mode at full high-definition resolution of 1920 x 1080 pixels at a frame rate of 24 frames per second (fps). The camera focus was manually set to a depth-of-field inline with the laser sheet, and the shutter speed was set to 1/400 $\mathrm{s}$ with an ISO of 1600 in order to minimize particle streaking while maximizing the sensitivity of the camera sensor to low light. The water tunnel was seeded with neutrally buoyant $11 \mu \mathrm{m}$ silver-coated glass spheres (Potters Industries ${ }^{\circledR}$ ) assumed to faithfully follow the fluid flow. Figure 4.5 shows a typical still frame exported from a video clip of particle-laden flow over a smooth coral model and illustrates the typical size of the field-of-view, the clarity of image focus, and the particle seeding density.

Because the video recording field-of-view was limited to $10 \mathrm{~cm}$ by $10 \mathrm{~cm}$, in order to capture the entire flow field over and downstream of the models, it was necessary to record three separate, slightly overlapping windows: one viewing the model's leading edge, one viewing the model's trailing edge, and one viewing the downstream wake. At each location, $60 \mathrm{~s}$ video data records were made for four different flow speeds: $2.5 \mathrm{~cm}$ $\mathrm{s}^{-1}, 5.0 \mathrm{~cm} \mathrm{~s}^{-1}, 10 \mathrm{~cm} \mathrm{~s}^{-1}$, and $15 \mathrm{~cm} \mathrm{~s}^{-1}$. Using the model diameter $D$ as the length scale for Reynolds number calculations, i.e., $R e=\frac{u_{\infty} D}{\nu}$, the flow rates corresponded to Reynolds numbers of 3375, 6750, 13500, and 20250, respectively. The incoming flow along the flat mounting plate allowed for the development of a laminar boundary layer before the flow interacted with the coral models. The thickness of the boundary 


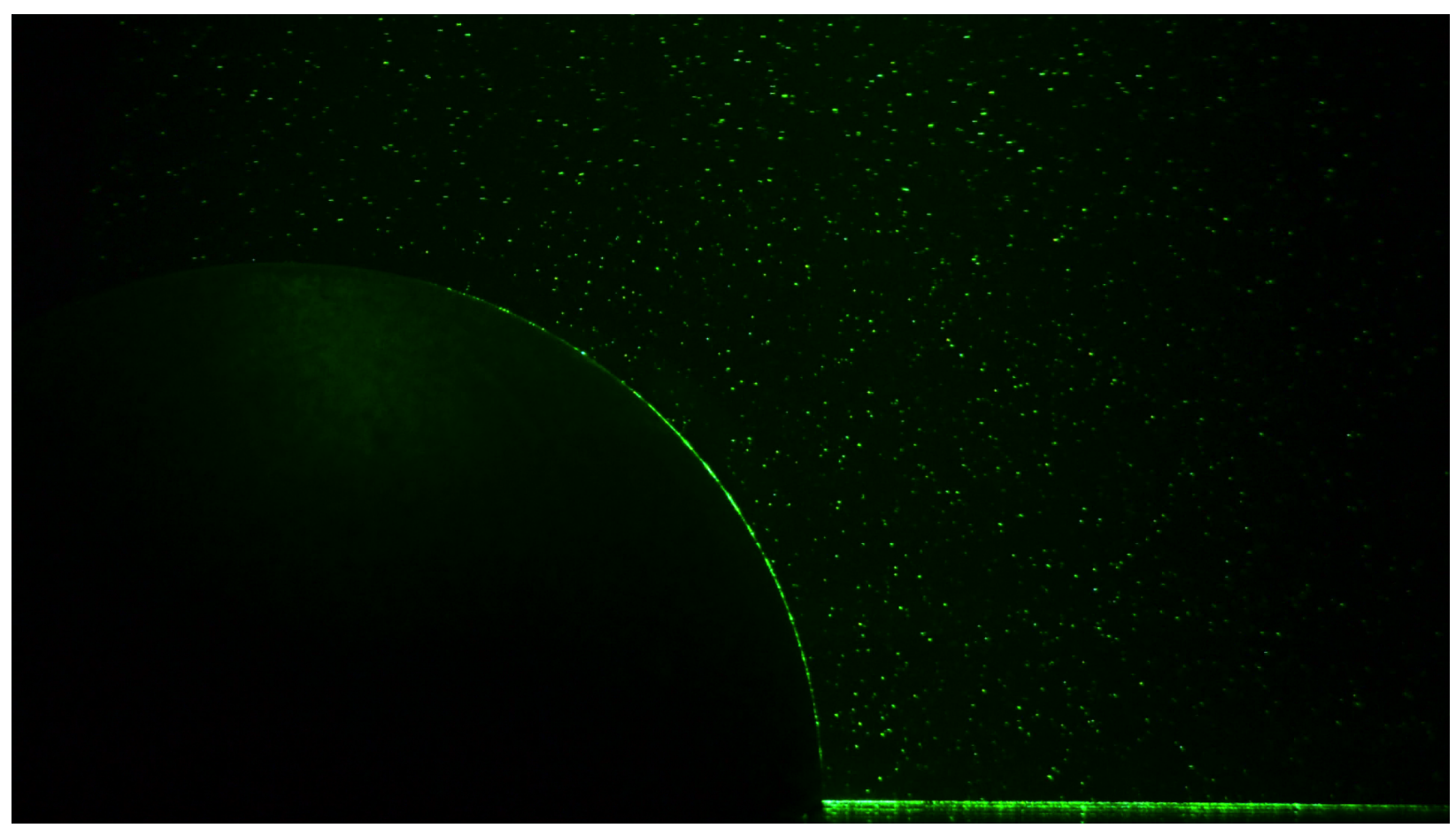

Figure 4.5: Individual still frame extracted from PIV video clip illustrating particle seeding, density, and focal clarity on the trailing edge of the smooth model. The free-stream horizontal velocity for this image is $15 \mathrm{~cm} \mathrm{~s}^{-1}$, which was most vulnerable to particle streaking due to camera exposure length.

layer of the incident flow varied from $\sim 0.7 \mathrm{~cm}$ to $\sim 1.7 \mathrm{~cm}$ as a function of the free-stream velocity, according to the Blasius solution for boundary layer flow over a flat plate, given as $\delta=5.0 \sqrt{(\nu x) / u_{\infty}}$, where $x$ is the distance downstream from the edge of the plate 61. Table 4.1 summarizes the experimental conditions tested using the PIV system.

Using the Matlab ${ }^{\circledR}$ Image Processing Toolbox (The MathWorks, Inc.), video records of raw PIV data were first converted to a series of 1440 individual still frames (24 fps for $60 \mathrm{~s}$ ) for use as paired images in the cross-correlation particle matching required to estimate velocities. Image pairs were interrogated using the open-source software MatPIV version 1.6.1 [105], which employs an iterative displacement estimating function derived from a hybrid digital particle image velocimetry/particle tracking velocimetry algorithm 119. If shear layers creating strong vertical gradients are present in the flow-as was expected-interrogation

\begin{tabular}{ccc}
\hline $\begin{array}{c}\text { Velocity } \\
{\left[\mathrm{cm} \mathrm{s}^{-1}\right]}\end{array}$ & $\begin{array}{c}\text { Reynolds Number } \\
{[-]}\end{array}$ & $\begin{array}{c}\text { Boundary Layer Thickness } \\
{[\mathrm{cm}]}\end{array}$ \\
\hline \hline 2.5 & 3,375 & 1.67 \\
5.0 & 6,750 & 1.18 \\
10.0 & 13,500 & 0.84 \\
15.0 & 20,250 & 0.68 \\
\hline
\end{tabular}

Table 4.1: Description of free-stream velocities, corresponding Reynolds numbers, and resulting flat-plate boundary layer thicknesses for experimental flows over coral models. 


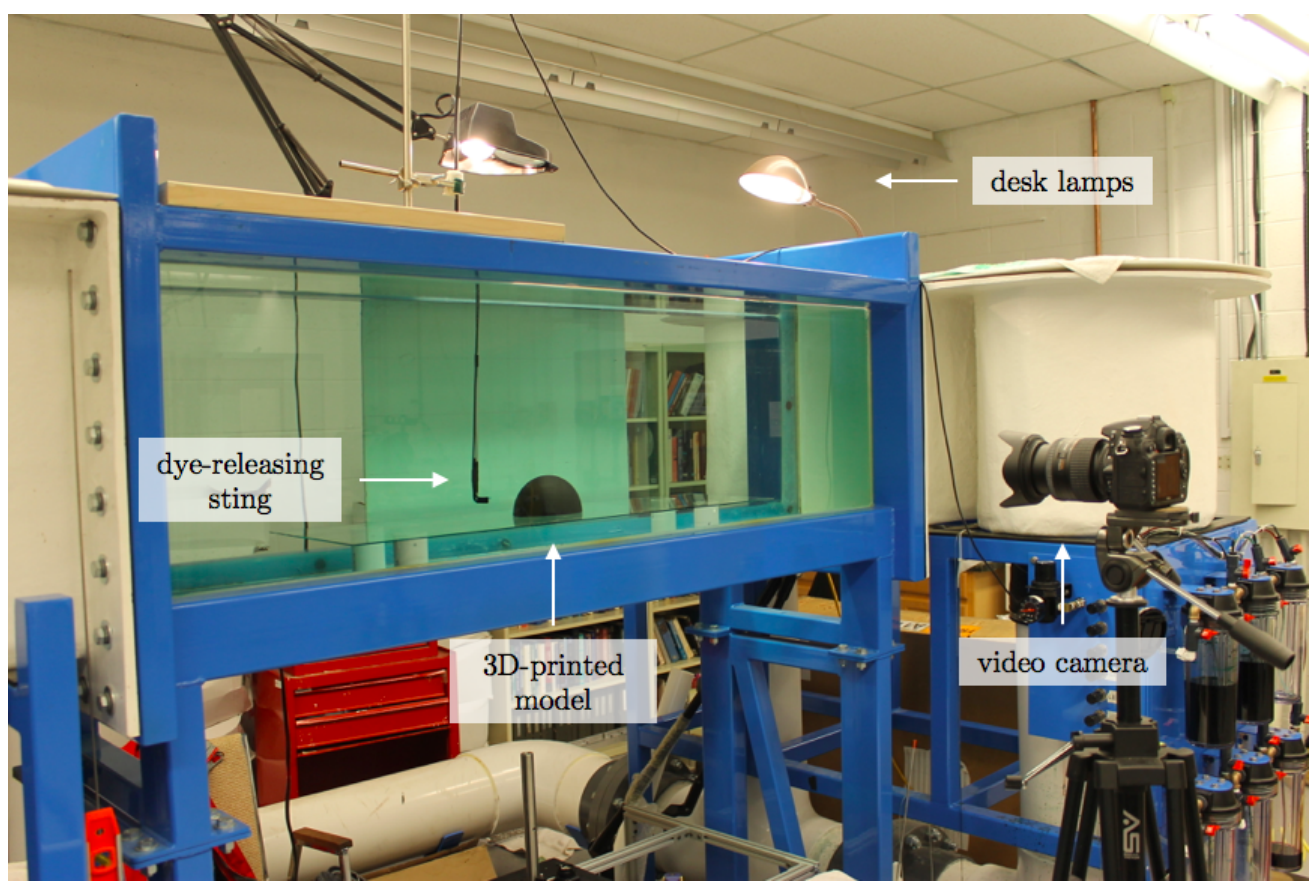

Figure 4.6: Photograph of setup for flow visualization using pressurized dye-releasing system.

windows that are too large in the vertical direction can create biased velocity values that overestimate the true velocity. To avoid this, initial interrogation windows with $75 \%$ overlap were implemented asymmetrically (with smaller vertical components) and decreased in size with subsequent passes until a symmetric final pass was used; the four window sizes implemented measured $256 \times 64$ pixels, $128 \times 32$ pixels, $64 \times 32$ pixels, and $32 \times 32$ pixels. For this window size and overlap, velocity estimates were produced at a final, physical resolution of approximately 0.75 by $0.75 \mathrm{~mm}$. Spurious vectors were identified using global mean and local median filters ( \pm 3 and \pm 2.5 standard deviations, respectively), and outliers were replaced using a linear interpolation of neighboring vectors.

\subsubsection{Dye Visualization Setup}

In addition to the quantitative data collected using PIV, a qualitative dataset describing flow over the coral models was obtained using the water tunnel's pressurized dye visualization system. The plate glassmounted models were positioned in the middle of the tunnel test section and illuminated from above by two 60-watt desk lamps. A 3/16 inch metal rod was clamped vertically to a metal stand positioned above the test section, and an L-shaped hex key was attached to the bottom of the metal sting with electrical tape, so that the release of dye was directed inline with the dominant horizontal flow path. Flexible Tygon ${ }^{\circledR}$ tubing with a diameter of $1 / 8$ of an inch was affixed with electrical tape to the metal sting and connected to the pressurized blue dye tank. The sting was positioned approximately 2.5 inches in front of the coral model 
and 1 inch above the glass mounting plate. Similar to the PIV setup, a Nikon D7000 DSLR camera was used in video recording mode to capture the dye visualization; however, the camera was positioned such that the entire model and downstream wake was visible in the field-of-view, eliminating the need for multiple recording windows. Video was recorded in full high-definition at $24 \mathrm{fps}$ with a shutter speed of $1 / 30 \mathrm{~s}$ and an ISO of 2500. Dye was released as a 10-second slug at a pressure that was intended to match the approximate flow speed, so as to not impart additional momentum to the dye-laden incoming flow. Visualizations were obtained for all four models at two flow speeds: $5 \mathrm{~cm} \mathrm{~s}^{-1}$ and $15 \mathrm{~cm} \mathrm{~s}^{-1}$. Figure 4.6 shows the experimental setup used to perform dye visualization over the models.

\subsection{Results: Mean Characteristics}

\subsubsection{Flow Visualization}

Qualitative differences in flow structure due to Reynolds number and surface roughness can be clearly identified through videos of dye visualization. Figure 4.7 plots a time series of still frames extracted from full-length video records for the smooth hemisphere at $R e=6,750$ (left), at $R e=20,250$ (middle), and for the k-type roughness at $R e=20,250$ (right). These three example cases were selected because they represent the most significant differences in flow speed and model roughness, and accordingly, they exhibited the largest differences in visualization results. Full video records for all model geometries and flow rates can be found in the appendix.

Examining first the different flow velocities past the smooth hemisphere (left versus middle), the main similarity between the two regimes is the vertical spread of the downstream wake, which extends to approximately one model radius, or $6.75 \mathrm{~cm}$, above the model top. Key differences are evident upon close examination of the wake structures, which show the low Re regime retains thin, high-concentration filaments of dye, whereas the high Re regime much more quickly "smears out" the wake-entrained dye into an homogenous, low-gradient area. The arrows in each frame mark the evolution of an individual packet of dye through the separation point and shear layer as it tracks the formation and dissolution of an individual eddy. The low Re regime shows evidence of hairpin vortex shedding, typical of von Kármán vortex shedding from spheres in low to moderate $R e$ flows [114]. The high $R e$ regime features more chaotic wake shedding without clear formation of coherent structures. The tracking arrows in both regimes show an individual eddy ripping apart with increasing distance between the pieces of the original eddy as time increases. Interestingly, after approximately the same distance downstream, $\sim 15 \mathrm{~cm}$, the eddy pieces are roughly the same distance apart for both regimes, though with the time elapsed being $3 \mathrm{x}$ longer in the low flow regime. 

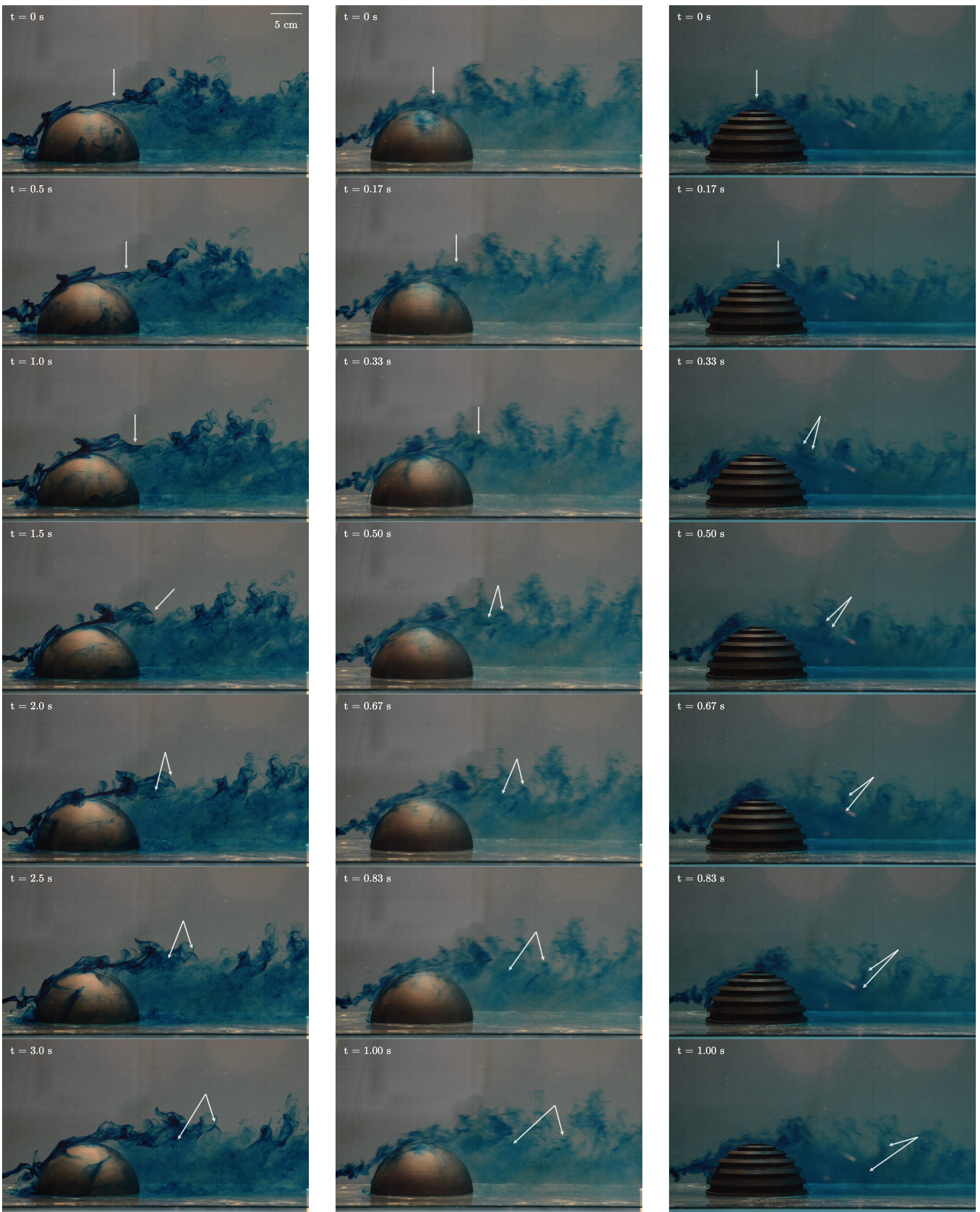

$\mathrm{t}=0.17 \mathrm{~s}$

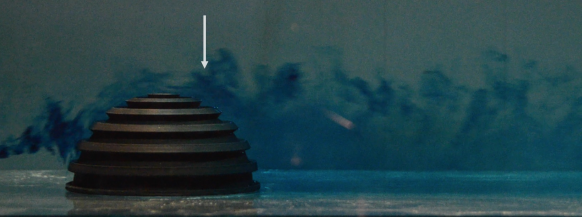

$\mathrm{t}=0.33 \mathrm{~s}$

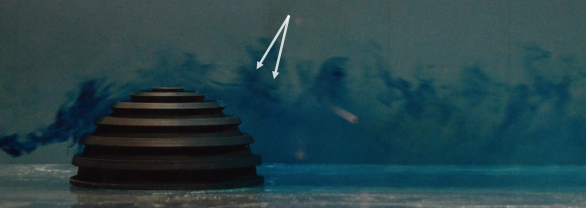

$\mathrm{t}=0.50 \mathrm{~s}$

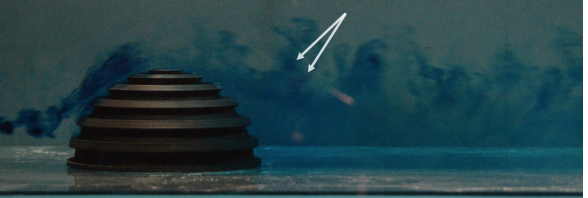

$\mathrm{t}=0.67 \mathrm{~s}$

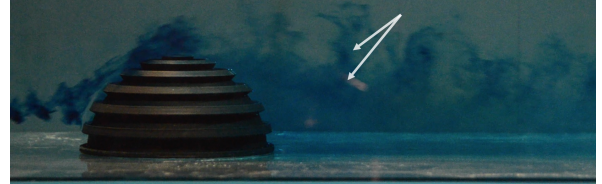

$\mathrm{t}=0.83 \mathrm{~s}$

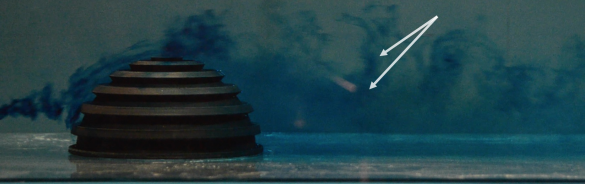

$\mathrm{t}=1.00 \mathrm{~s}$

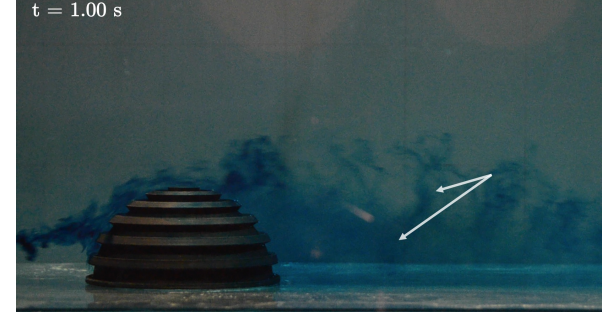

Figure 4.7: Left column. Dye visualization of flow past a smooth hemisphere at $5 \mathrm{~cm} \mathrm{~s}^{-1}$, i.e., $R e=6,750$. Each descending row is a still frame from the video record separated by $0.5 \mathrm{~s}$, or 12 frames. Middle column. Flow past smooth hemisphere at $15 \mathrm{~cm} \mathrm{~s}^{-1}$, i.e., $R e=20,250$. Each row is separated by $0.17 \mathrm{~s}$, or 4 frames. Right column. Flow past hemisphere with k-type roughness at $15 \mathrm{~cm} \mathrm{~s}^{-1}$, i.e., $R e=20,250$. Row spacing is $0.17 \mathrm{~s}$, or 4 frames. 
Comparing the high Re cases for the smooth (center) and k-type (right) models reveals a key difference in the size of the downstream wake. Whereas the smooth model wake extends vertically from the bed to approximately double the model height, the k-type model wake briefly extends approximately $3 \mathrm{~cm}$ above the model top and quickly is directed downward so that by the end of the recording frame, the vertical extent of the wake is no higher than the model itself. Similar to the smooth model, the k-type also "smears out" high-gradient filaments of dye very quickly, indicating rapid scalar mixing within the chaotic wake. Finally, the dissolution of an individual dye-containing eddy into several pieces appears to occur at nearly the same rate and results in a similar distance of separation between the two models, suggesting a similar rate of energy dissipation controlling the inertial cascade.

\subsubsection{Mean Horizontal Velocity}

Figure 4.8 plots the mean horizontal velocity for the low-flow case of $u_{\infty}=5 \mathrm{~cm} \mathrm{~s}^{-1}$ (left) and for the high-flow case of $u_{\infty}=15 \mathrm{~cm} \mathrm{~s}^{-1}$ (right). All subsequent PIV results panels use the same layout as Figure 4.8, i.e., Row 1 is the smooth hemisphere, Row 2 is the d-type roughness, $w / k=0.5$, Row 3 is the k-type roughness, $w / k=2.0$, and Row 4 is the coral skeleton. Each individual panel is stitched together from four separate recordings of the model's leading edge, center, trailing edge, and downstream wake. To create a single portrait of the entire flow field around the models, overlapping regions from each window were cropped at matching locations (indicated by the vertical white spacing at $x=-1.5 \mathrm{~cm}, x=1.5 \mathrm{~cm}$, and $x=10 \mathrm{~cm}$ for the idealized models and at $x=-4 \mathrm{~cm}, x=3 \mathrm{~cm}$, and $x=9 \mathrm{~cm}$ for the coral skeleton).

Several key differences are evident in the mean flow characteristics between flow conditions and across model geometries. First, comparing the leading edges of the low and high flow regimes, the $15 \mathrm{~cm} \mathrm{~s}^{-1}$ flow creates a significant region of recirculating negative flow, which extends approximately $1-2 \mathrm{~cm}$ normal to the model face for all geometries. The $5 \mathrm{~cm} \mathrm{~s}^{-1}$ flow exhibits reduced positive flow along the leading edge, but no negative velocities that would indicate an area of recirculation. Comparing differences across geometry types, little difference is seen in the leading edges of the high flow case, except for increased patchiness for the k-type and coral models. However, the low flow panels show a clear effect of surface topography, in which the k-type roughness possesses distinct regions of reduced velocities just above individual roughness cavities, indicating the ejection of low speed vortices that disrupt the leading edge boundary layer. Close inspection of the d-type roughness and coral model reveal similar bulges of low momentum fluid being ejected from the roughness cavities, though with a smaller vertical range compared to the k-type roughness. No such disruptions affect the leading edge of the smooth model.

Next, examining the trailing edges and downstream wakes illustrates the large effect of Reynolds 
(a)

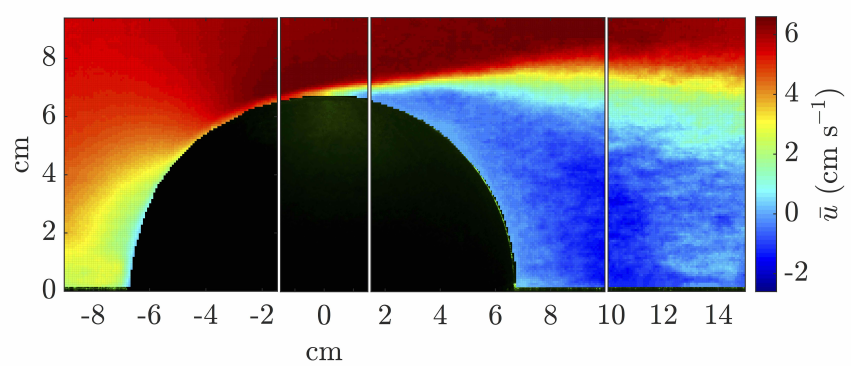

(b)

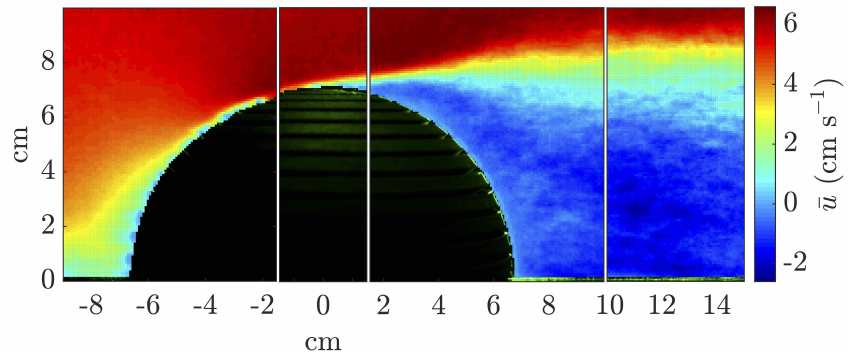

$(c)$

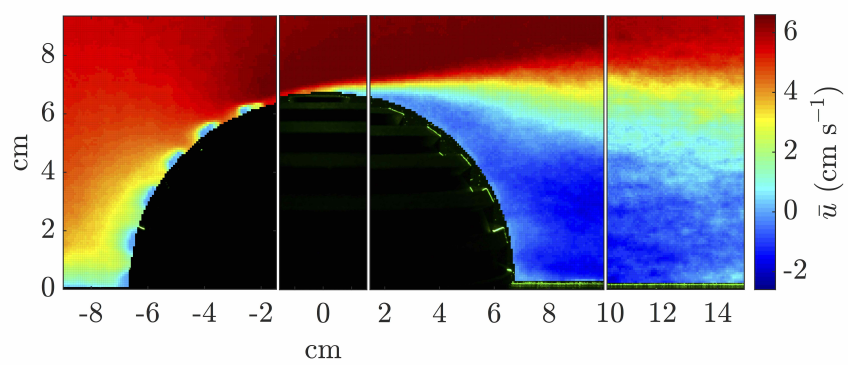

$(d)$

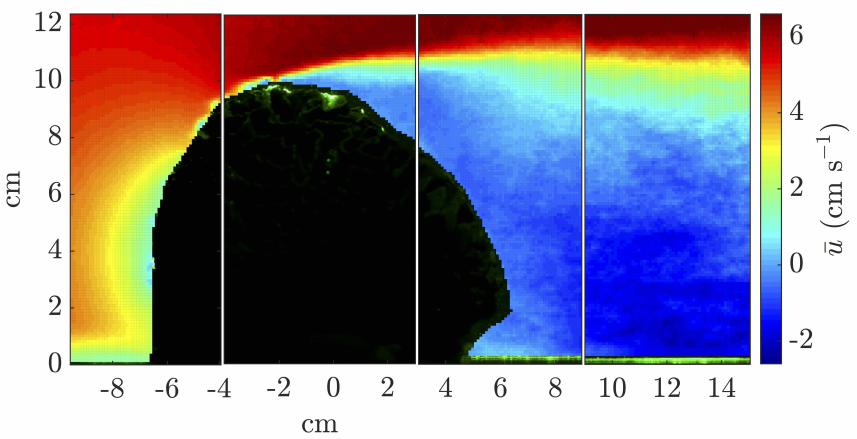

(e)

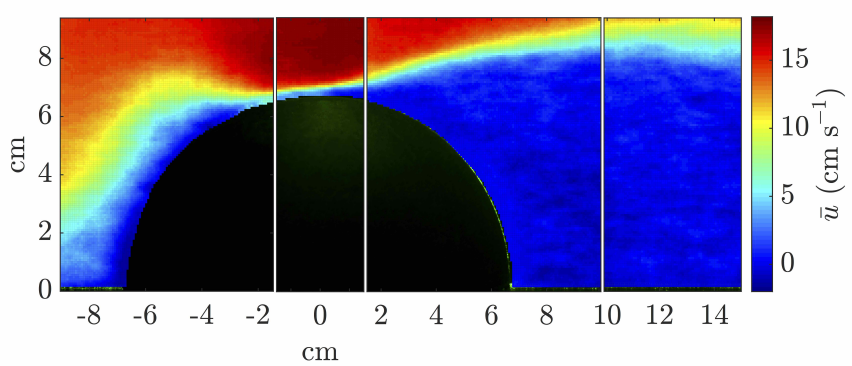

$(f)$

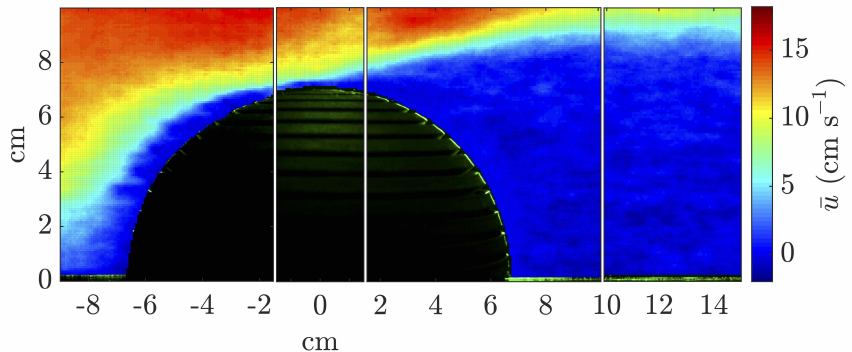

$(g)$

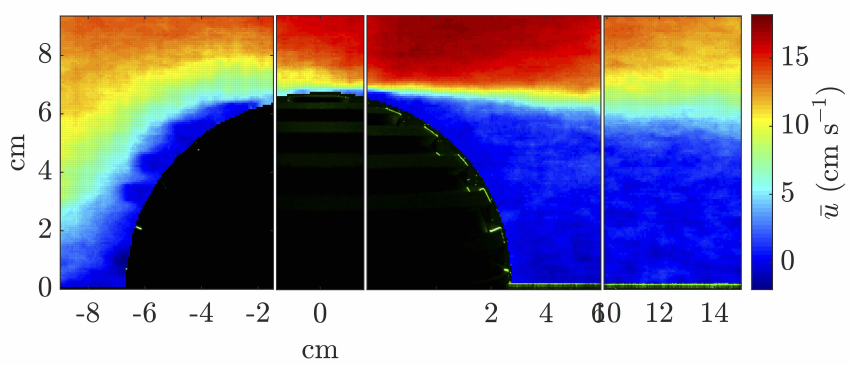

$(h)$

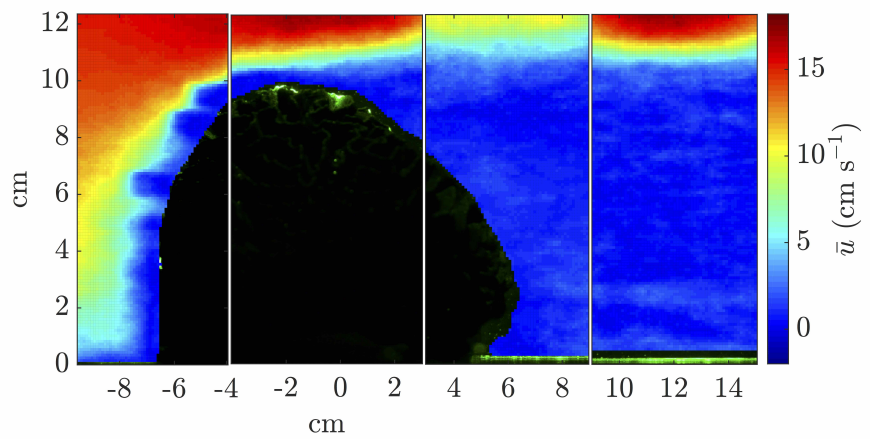

Figure 4.8: Distribution of mean horizontal velocity $\bar{u}$. Left column. Low-flow condition: $u_{\infty}=5 \mathrm{~cm} \mathrm{~s}^{-1}, R e=6,750$. Right column. High-flow condition: $u_{\infty}=15 \mathrm{~cm} \mathrm{~s}^{-1}, R e=20,250$. Row 1. Smooth hemisphere. Row 2. Hemisphere with d-type roughness, $w / k=0.5$. Row 3. Hemisphere with k-type roughness, $w / k=2.0$. Row 4. Coral skeleton, $w / k \sim 1$. 
number on the distribution patterns of mean velocity. The high-flow panels all feature very large zones of recirculation, which encompass nearly the entire downstream imaging windows. The most significant difference is in the k-type roughness (panel $g$ ), in which the downstream shear layer is directed downward toward the bed, rather than upward as is seen in the smooth and d-type roughness (panels $e$ and $f$ ). This redirection of the wake shear layer results from a delay in the flow separation point, achieved by the injection of momentum from leading edge roughness elements in order to overcome the adverse pressure gradient. The coral model exhibits behavior in-between these two extremes, such that the shear layer is nearly parallel to the bed. The low-flow panels all exhibit relatively similar distributions downstream, with a much smaller recirculation zone and a much less step vertical gradient in the shear layer compared to the high-flow panels.

\subsubsection{Polar Separation Angle}

In all panels of Figure 4.8, the flow on the trailing edge diverges from the model surface creating a separation point in the boundary layer. The location of this separation point determines the magnitude of the polar separation angle $\Theta_{S}$, which is measured from the bed at the leading edge to the point of flow separation-identified by locating the first negative velocity measurement near the surface of the model's trailing edge. Figure 4.9 plots the polar separation angle for each model under low $R e$ and high $R e$ conditions. For all model types, $\Theta_{S}$ is greater under the high Reynolds number condition, indicating a delay in flow separation at higher free-stream flow rates, a result consistent with previous literature [112]. The smooth and d-type roughness models exhibit very similar behaviors, as previously reflected in the mean velocity plots of Figure 4.8, suggesting the tightly packed roughness elements of the d-type model allow the boundary layer flow to predominantly skim over the roughness cavities without significantly altering the large-scale flow characteristics.

The polar separation angle of the k-type model under low flow, in contrast, is greater than $\Theta_{S}$ for all models regardless of flow condition. Further, the k-type model under high flow exhibits a greater than 25\% increase in $\Theta_{S}$ compared to the low-flow condition, which corresponds to an additional $3.3 \mathrm{~cm}$ of curvilinear contact along the midline between the model surface and the high-momentum boundary layer (given the model radius of $6.75 \mathrm{~cm}$ ). Estimating $\Theta_{S}$ for the coral model is difficult due to the coral's irregular shape, which makes defining a centerline and corresponding polar angle a subjective task. However, regardless of the accuracy of the absolute magnitude of $\Theta_{S}$, the relative magnitudes between the low-flow and high-flow cases provides valuable information. For the coral model, the high-flow case produces an $18 \%$ larger polar separation angle - a value in between the smooth/d-type models and the k-type model, which further reflects the intermediate $w / k$ roughness ratio of the coral skeleton. 


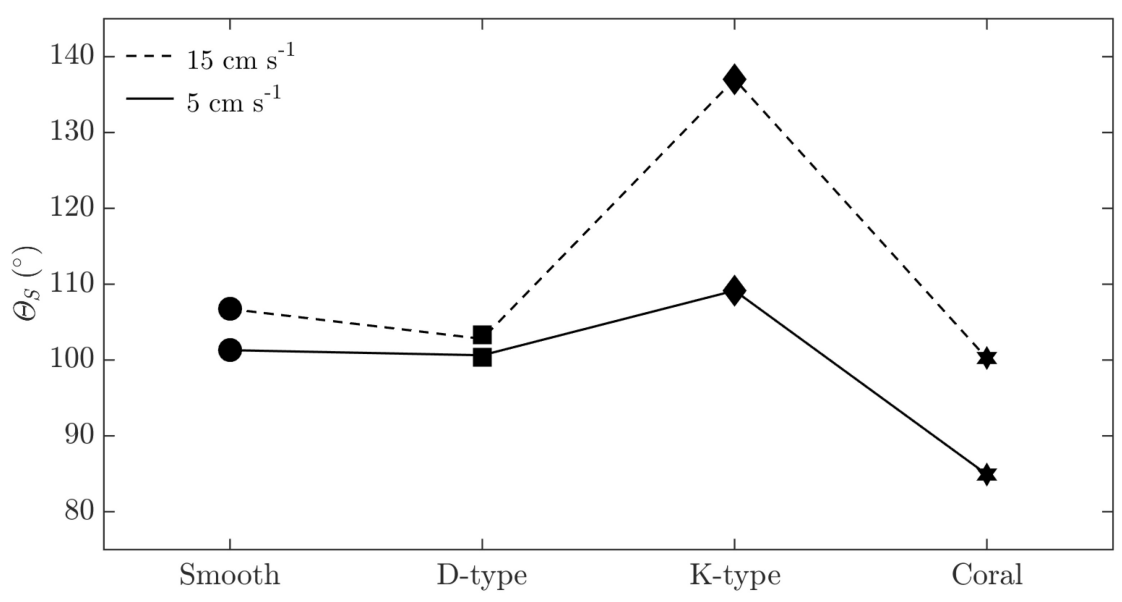

Figure 4.9: Polar separation angle for each model.

\subsubsection{Streamlines}

Streamlines of the mean velocity field were calculated for each model under low- and high-flow conditions, according to the definition $\mathbf{u} \times d \mathbf{s}=0$, where $d \mathbf{s}$ is a small element of the arc length along a streamline. Individual panels for the leading edge, trailing edge, and downstream wake were calculated separately and pieced together during post-processing to create a single image of streamlines for each model. Due to the need to select new streamline starting points on the boundaries of each panel, some small discrepancies exist at the junction points between the three panels for each model.

The left column of Figure 4.10 (panels $a-d$ ) plots the streamlines for the low-flow regime at $R e=$ 6,750 . The leading edges of each model show nearly identical results with a stagnation point at approximately $z=2 \mathrm{~cm}$ (slightly higher for the taller coral model at $z=3.5 \mathrm{~cm}$ ) and smooth, undisturbed flow leading to significant streamline compression from approximately $z=5 \mathrm{~cm}$ to the model top, which indicates an area of large negative (suction) pressure. The trailing edges and downstream wakes, by contrast, highlight several important differences created by the model geometries. Streamlines of each model's large-scale wake appear topologically similar and demonstrate the presence of a closed toroidal vortex, whose center location varies according to model roughness. The k-type model produces a downstream vortex closest to the model surface, located at $x=9.5 \mathrm{~cm}, z=5 \mathrm{~cm}$, which holds significant implications for near-surface mixing and mass flux. Interestingly, the smooth model also possesses two secondary vortices on the trailing edge, known as Moffat vortices 120, which are typically found at corners and near points of flow separation as is the case here. Such secondary vortices do not appear on the d-type, k-type, and coral models, presumably having been disrupted by the injection of momentum generated by the surface topographies.

Streamlines for the high-flow regime at $R e=20,250$ are shown in the right column of Figure 4.10 
(a)

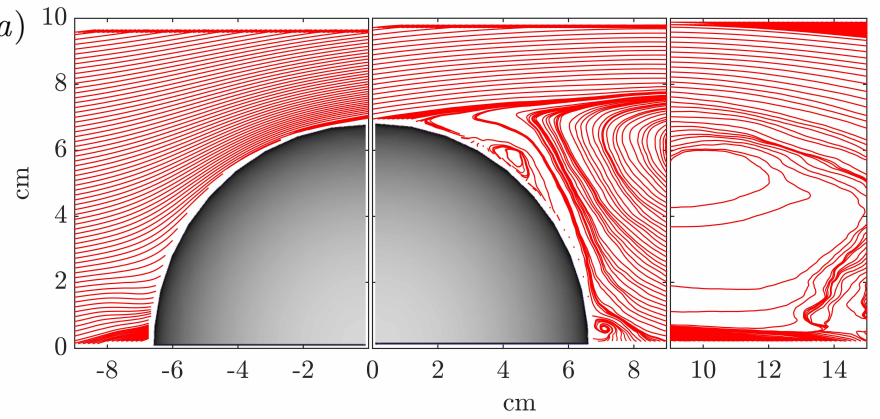

(b)

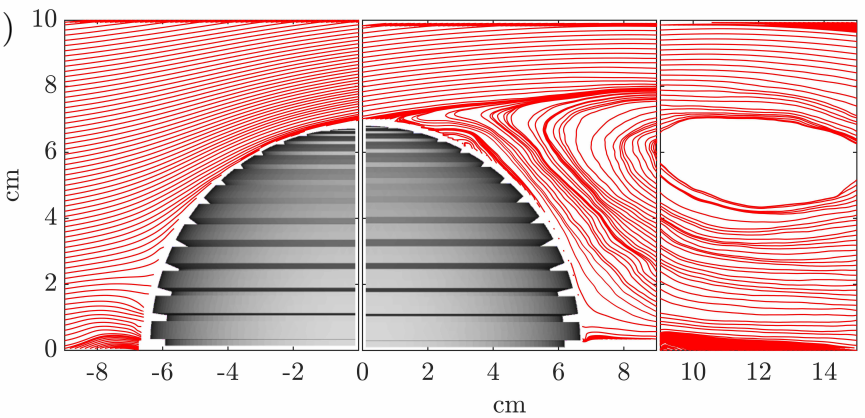

$(c)$

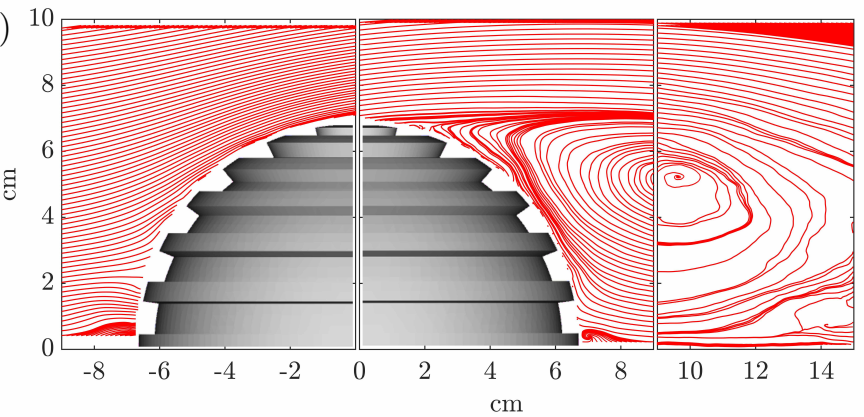

(d)

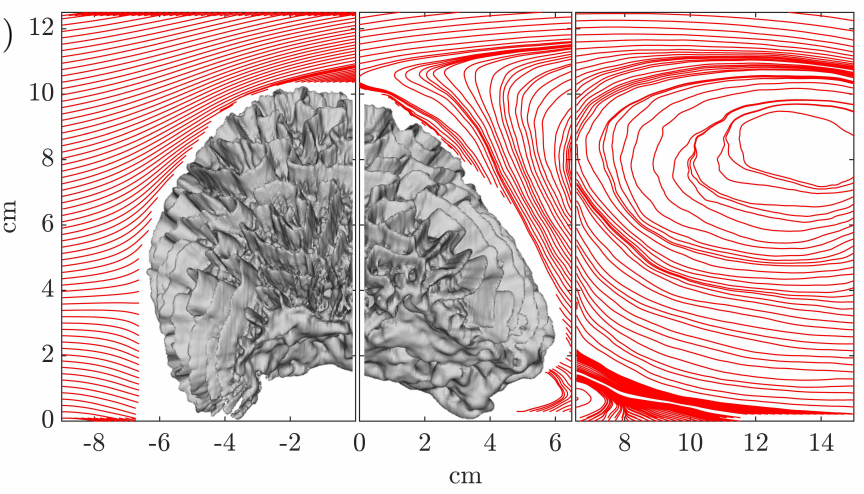

(e)

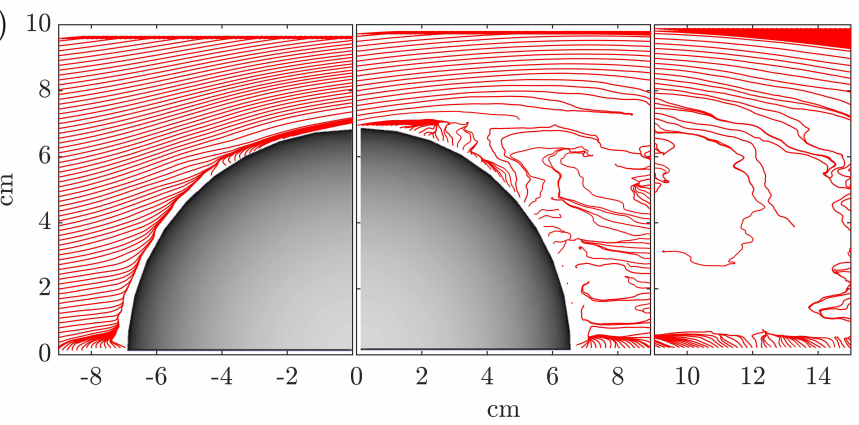

$(f)$

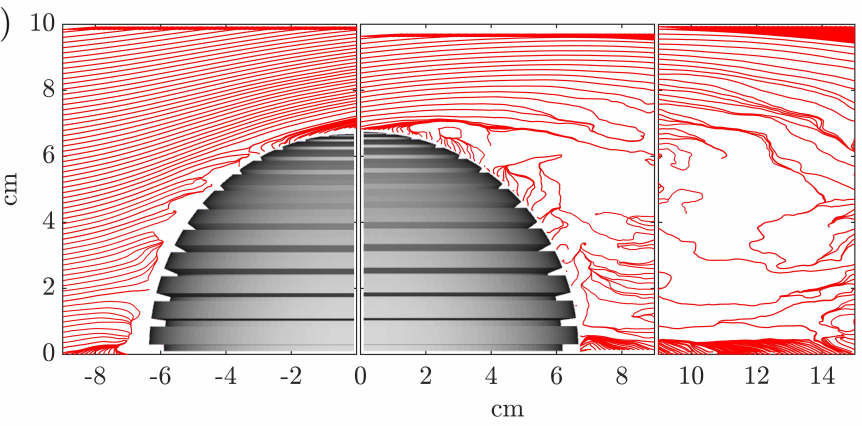

$(g)$

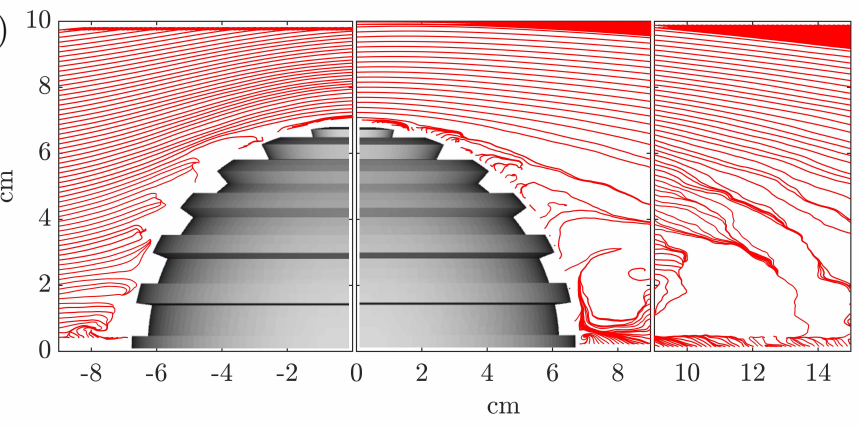

(h)

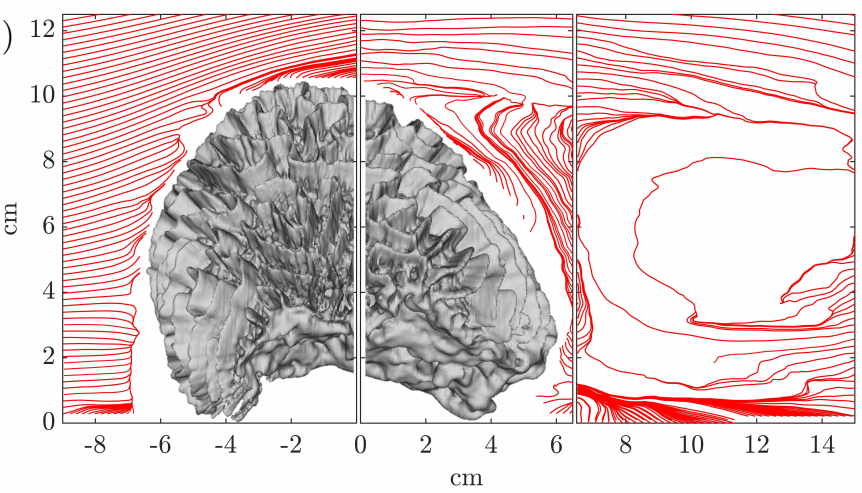

Figure 4.10: Streamlines of mean horizontal velocity for $R e=6,750$ (left column) and $R e=20,250$ (right column). 
(panels $e-h$ ). Unlike the low-flow regime, the leading edge streamlines show significant differences among model geometries. The smooth model begins to demonstrate minor unsteadiness in the flow paths near the model surface, but the streamlines remain intact. The d-type, k-type, and coral models all exhibit interrupted streamlines along the leading edge, as the incident flow interacts with the large-scale roughness elemednts to create pockets of local recirculation. The magnitude of the leading-edge disturbance outward from the model surface correlates well with $w / k$ ratio, such that the tightest roughness spacing of the d-type model disturbs the flow less than the roughest spacing of the k-type model. Streamlines along the trailing edge of each model are heavily disrupted by the turbulent wake and show little difference among the models. The most significant difference is the much smaller area of chaotic streamlines present behind the k-type model, which is reflective of the delay in flow separation compared to the other models and the downward trajectory of the wake as seen in Figure 4.8 .

\subsubsection{Vorticity}

Turbulent flows are by definition rotational, and the measure of the local rotation within a fluid continuum is given by the flow's vorticity. As a key descriptor of eddy strength, vorticity is twice the angular velocity of a local fluid region and given by the curl of the velocity, i.e., $\boldsymbol{\omega} \equiv \nabla \times \mathbf{u}[121$. Mean horizontal and vertical flow fields were used to estimate the two-dimensional vorticity field over each model geometry, and the results are shown in Figure 4.11. The left column $(a-d)$ illustrates the vorticity fields for $R e=6,750$ and demonstrates very little difference in the spatial distribution of high vorticity regions among the four geometries. Each model shows peak levels of vorticity near the model top, approximately at the point of flow separation where the axisymmetric shear layer develops, and a rapid decline in magnitude in the streamwise direction. In addition to similar distribution patterns, maximum vorticity levels within the shear layer of each model are very close, ranging from $-14.77 \mathrm{~s}^{-1}$ for the smooth hemisphere to $-15.91 \mathrm{~s}^{-1}$ for the k-type geometry. The leading edges of the d-type, k-type, and coral models show clear evidence of vortical structures ejecting from roughness cavities. Interestingly, the strength of these vortices is roughly $25 \%$ greater than the vorticity generated by the shear layer, ranging from -18.39 to $-19.79 \mathrm{~s}^{-1}$.

The right column of Figure 4.11 $(e-f)$ shows the vorticity distribution for $R e=20,250$. In contrast to the low flow regime, the shear layer distribution pattern is not identical across all model geometries. Rather, the smooth and d-type models show a layer of strong vorticity projecting slightly upward - matching the trajectory of the shear layer in the mean horizontal velocity of Figure 4.8 -while the k-type vorticity is directed downward, and the coral vorticity is directed nearly parallel to the bed. Similar to the low flow regime, however, the d-type, k-type, and coral models all show the ejection of vortices from the leading edge, 
(a)

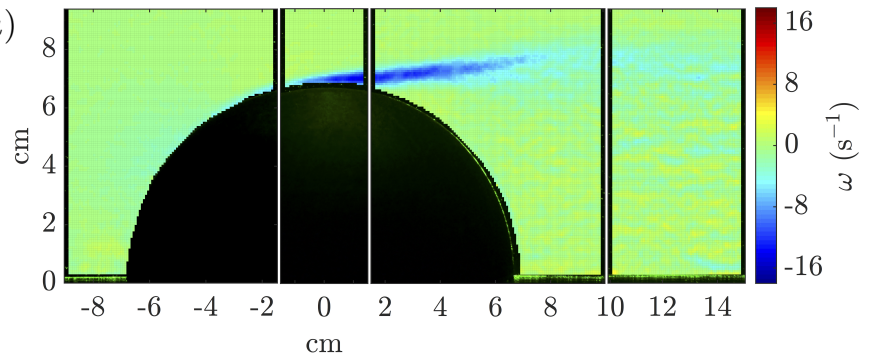

(b)

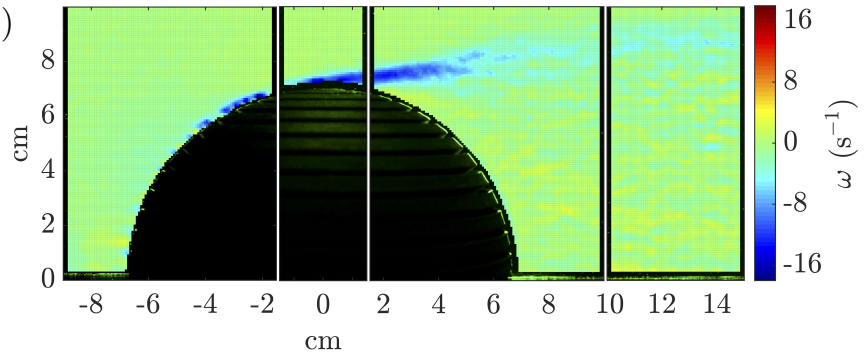

(c)

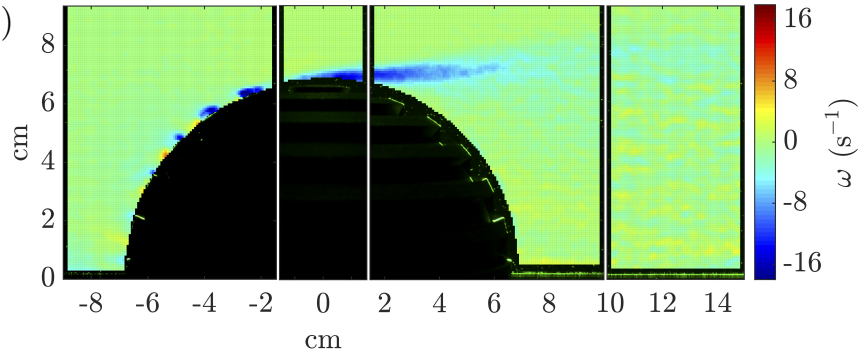

(d)

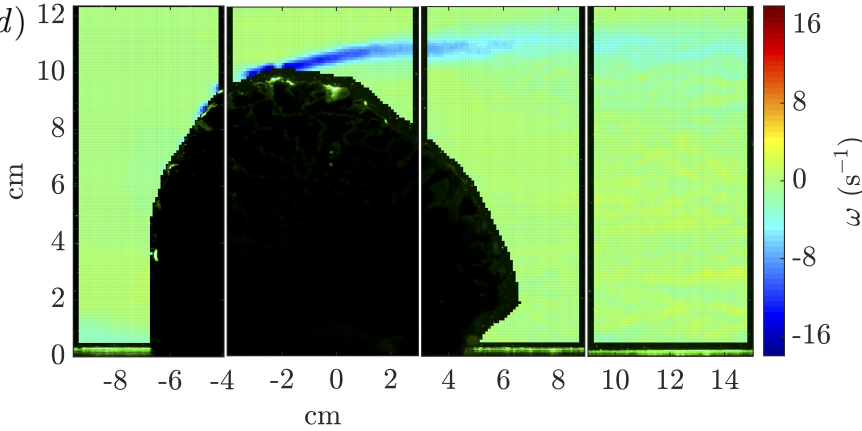

$(e)$

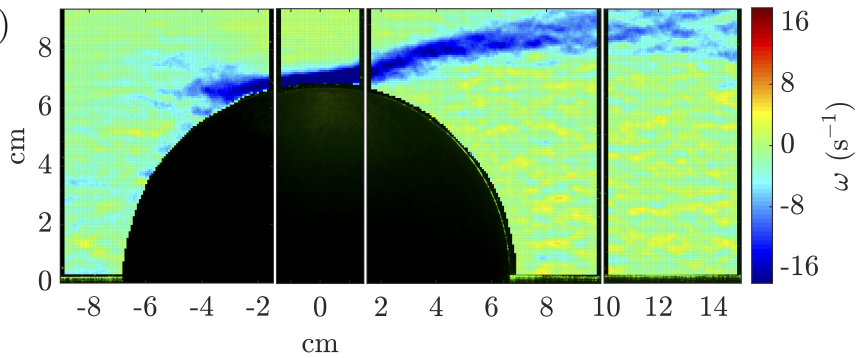

$(f)$

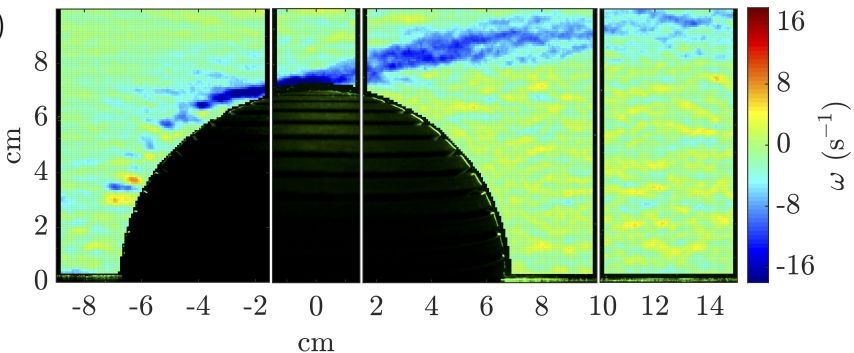

$(g)$

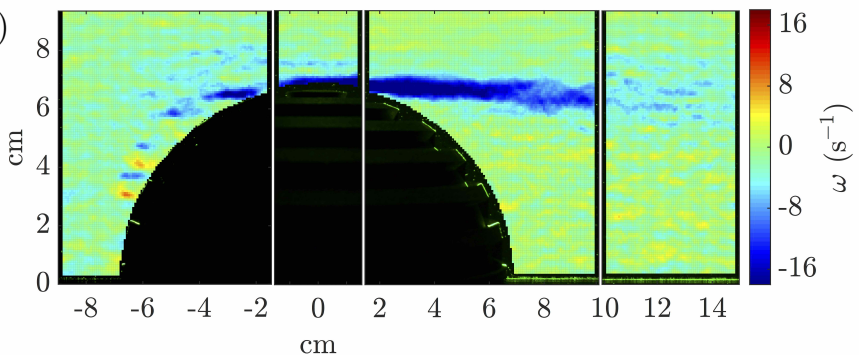

(h)

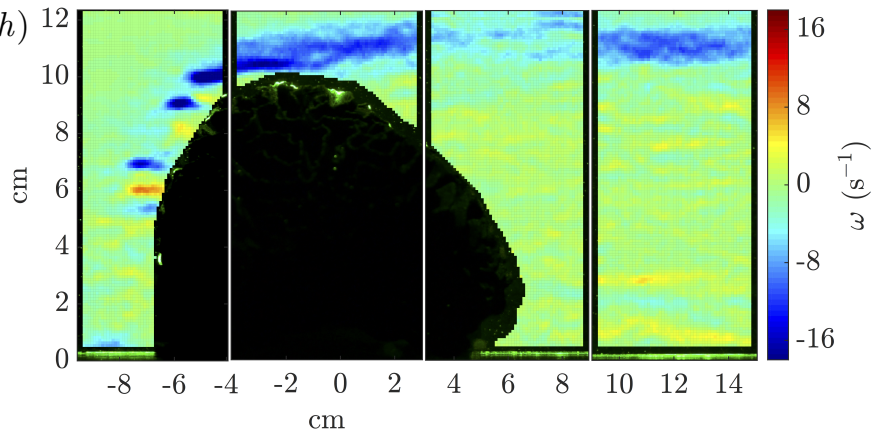

Figure 4.11: Vorticity plots for low Re regime (left column) and high Re regime (right column). Row 1 is smooth model, row 2 is d-type roughness model, row 3 is k-type roughness model, and row 4 is coral skeleton model. 
which the smooth model lacks. Interestingly, whereas the low flow regime featured stronger vorticity ejected from the leading edge compared to the wake shear layer, for the high flow regime this pattern is reversed. Peak vorticity levels in the shear layer range from -25.36 to $-43.62 \mathrm{~s}^{-1}$ and are reduced between $10-40 \%$ along the leading edge, with the greatest reduction occurring over the smooth model. Unlike the low flow regime in which the smooth model produces the smallest vorticity, for the high flow regime the shear layer behind the smooth model generates by far the highest levels of vorticity-approximately $25 \%$ greater than the other models - which suggests that the disruption of the shear layer due to leading edge vortices for the rough models interferes with shear-layer vortex production.

\subsection{Results: Turbulence Characteristics}

\subsubsection{Reynolds Shear Stress}

Reynolds shear stress provides a measure of momentum transfer from mean flow resulting from turbulent fluctuations and is a key descriptive characteristic of turbulence with a direct role in mass flux and mixing. When calculating Reynolds stresses, a standard Reynolds decomposition is performed for each velocity component, i.e., $u^{\prime}=u_{i}-\bar{u}$. For wall-bounded shear flows, the mean streamwise velocity component is typically parallel to the flat wall, so that Reynolds stresses provide information regarding the flux of momentum and mass toward (i.e., $-\overline{u^{\prime} w^{\prime}}$ ) or away (i.e., $+\overline{u^{\prime} w^{\prime}}$ ) from the interface. However, since the models possess curved surfaces, the Eulerian frame of reference requires a rotation of coordinate systems into the dominant flow direction in order to characterize the fluxes normal to the coral surface. Coordinate rotation is only required, however, for the leading edge until the point of flow separation since these surface areas are interacting with the incoming flow. On the trailing edge and in the wake region, no such rotation is necessary as the flow becomes a free shear layer without reference to a surface.

Figure 4.12 shows a comparison of Reynolds stresses calculated with and without coordinate rotation into the dominate flow direction. Reynolds stresses are non-dimensionalized by the square of the free-stream velocity $u_{\infty}^{2}$ and indicated by an asterisk. Without rotation (left panel), the cross-correlation of fluctuating velocity components produces unphysical positive Reynolds stress values in the region of flow compression at the leading edge. By contrast, coordinate rotation in the wake region creates unrealistically large Reynolds stress values also with an unphysical distribution pattern (right panel). Thus, to accommodate both the leading edge wall-bounded shear layer and the trailing edge free shear layer, Reynolds stress plots were constructed by combining coordinate rotation for the leading edge and center two panels with no rotation for the trailing edge and downstream panels. 

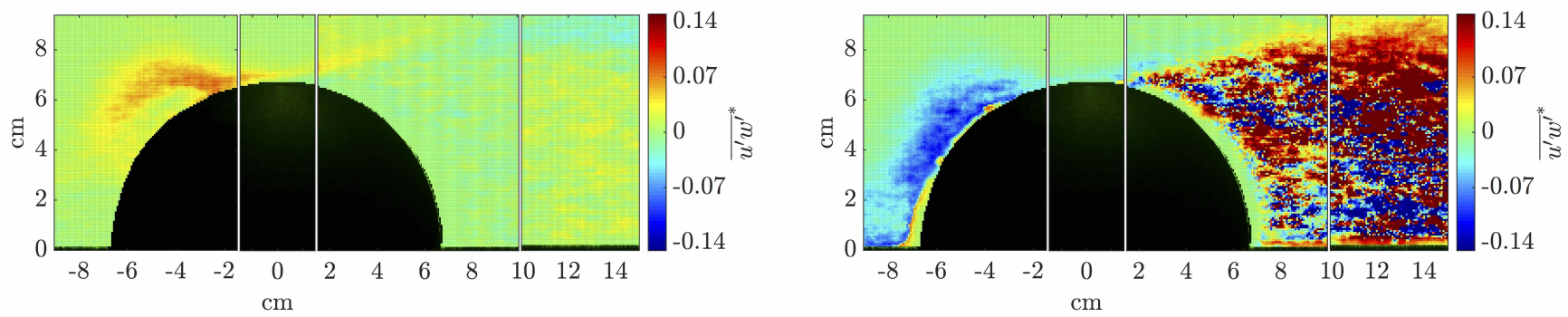

Figure 4.12: Normalized Reynolds shear stress plots for smooth hemisphere at $15 \mathrm{~cm}$ with coordinate rotation into dominant flow direction for all panels (left) and without coordinate rotation for all panels. (right).

Figure 4.13 plots distributions of normalized Reynolds stress ${\overline{u^{\prime} w^{\prime}}}^{*}$ for each roughness type under low-flow (left) and high-flow (right) conditions. First, the leading edge shows a large contrast in distribution and magnitude between the low-flow regime, which exhibits very little Reynolds stress, and the high-flow regime, which exhibits broad and intense shear stresses normal to the model surface. The low flow regime does show a "hot spot" of Reynolds stress in the recirculation zone, where each model meets the glass mounting plate. Values are positive in this area because the recirculating flow is moving in the negative direction of the prescribed coordinate system. This "hot spot" is noticeably absent from the coral model (panel $d$ ) because the recirculation is occurring in the shadow of the protruding edge, where PIV data was unable to be collected.

The distributions of $\overline{u^{\prime} w^{\prime}}{ }^{*}$ over the leading edges for the high $R e$ regime closely follow the patterns of mean flow parameters seen previously. In particular, the smooth model (panel $e$ ) exhibits peak stresses very near the surface, while the d-type (panel $f$ ), k-type (panel $g$ ), and coral (panel $h$ ) models all have low stress buffer regions adjacent to the surface, which force peak Reynolds stress farther out normal to the curved surface. The widely spaced roughness elements of the k-type model demonstrate clear spatial effects, with ejection of high intensity Reynolds stresses occurring from the roughness cavities. Quantitatively, peak values of normalized $\overline{u^{\prime} w^{\prime}}$ for the smooth, d-type, and coral model all range between -0.12 to -0.13 , whereas the k-type roughness exhibits approximately $35 \%$ higher peak stresses of -0.17 at the leading edge.

Comparison of distribution patterns and intensities of Reynolds stresses for the trailing edge and downstream wake regions exhibit an interesting and nearly reverse scenario from the leading edge. For all model types, the wake regions under the low-flow regime possess nearly $2 \mathrm{x}$ larger peak Reynolds stresses, ranging from -0.09 to -0.14 , compared to the high flow regime, which range from -0.04 to -0.07 . Though counterintuitive at first glance, the larger turbulent stress for the low Reynolds number regime reflect the contribution from coherent structures that disappear in the high Reynolds number chaotic wake. For all plots in figure 4.13, the wake regions show negative Reynolds stresses near the top of the recording window, where mean flow is from left-to-right, and positive Reynolds stresses below the shear layer in the recirculation 
(a)

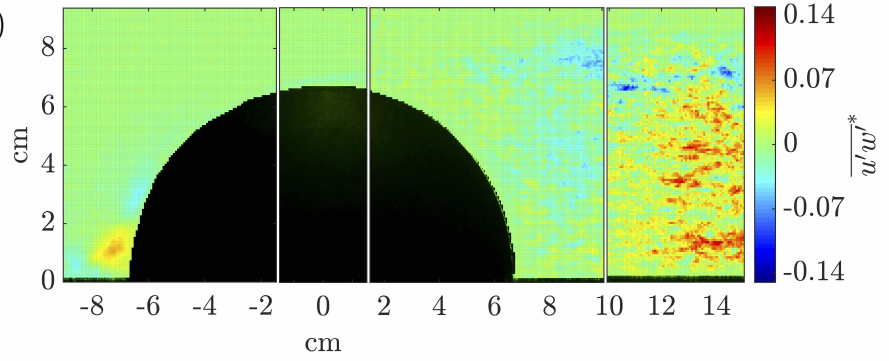

(b)

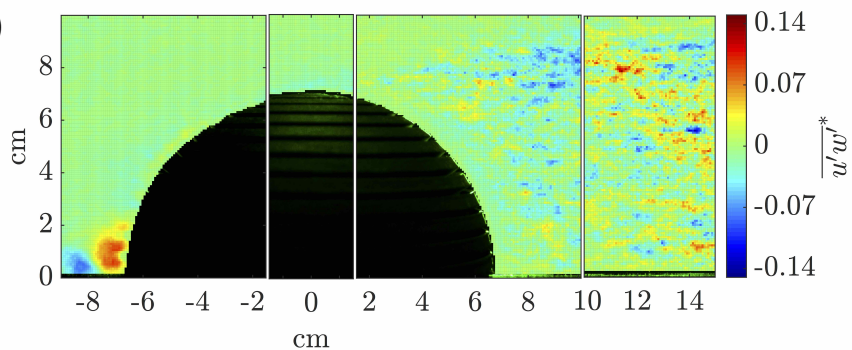

(c)

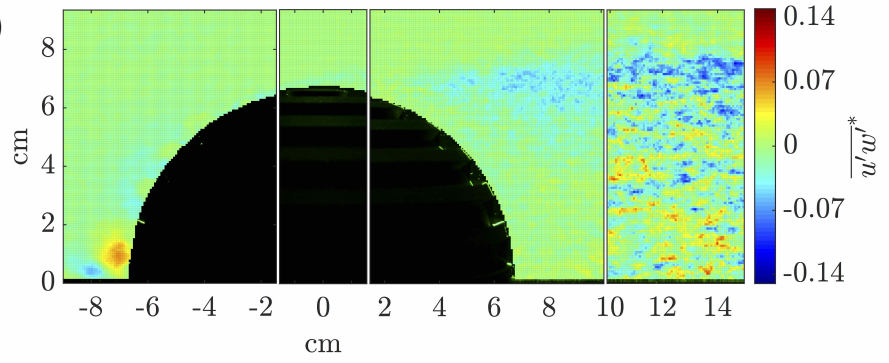

(d)

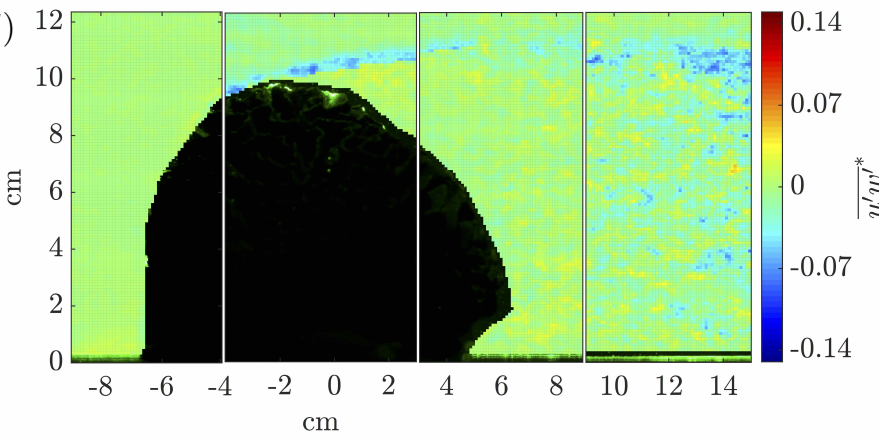

(e)

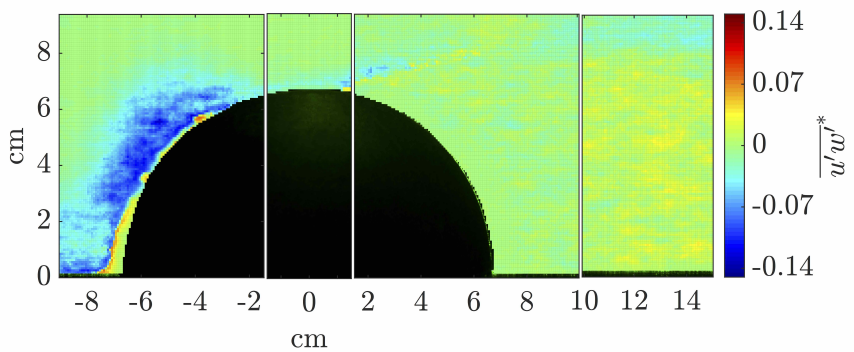

$(f)$

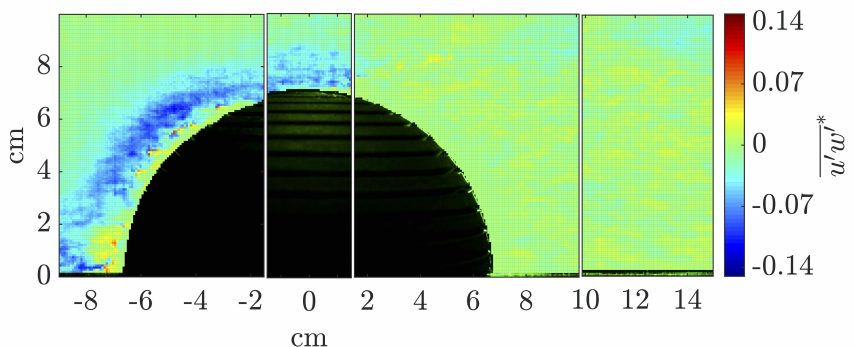

$(g)$

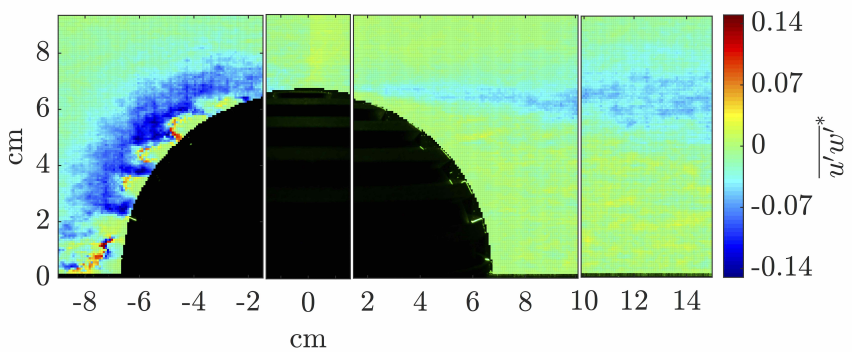

(h)

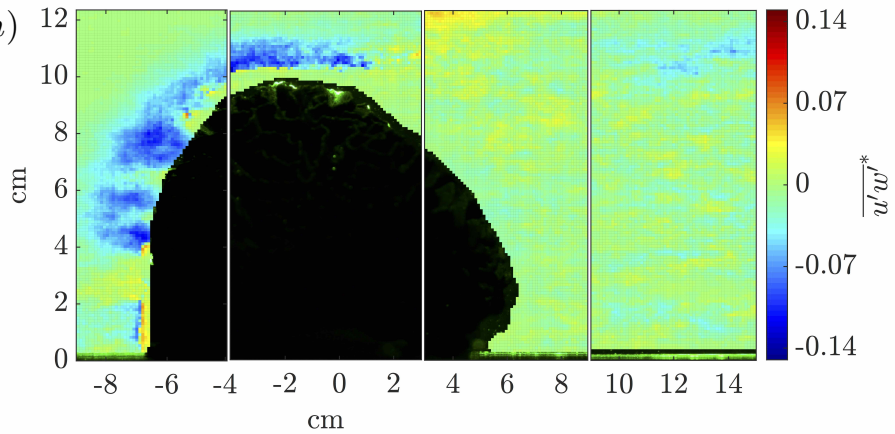

Figure 4.13: Normalized Reynolds shear stress plots for $R e=6,750$ (left column) and for Re $=20,250$ (right column). Row 1 is smooth model, row 2 is d-type roughness model, row 3 is k-type roughness model, and row 4 is coral skeleton model. 
zone, where mean flow is right-to-left. Distribution patterns and normalized magnitudes measured here are consistent with those observed in wakes behind spheres in the laboratory $[122$ and in wakes behind coral bommies in the field 123. Finally, the most significant observation regarding the effect of morphology on wake Reynolds stresses is the much broader distribution and much larger magnitude $(\sim 2 \mathrm{x})$ of Reynolds stresses resulting from the k-type morphology. The region of Reynolds stresses is directed downward due to the delay in flow separation, and the vertical spread of stresses is much wider, reflecting the broader vertical gradient in mean velocity of the downstream shear seen in figure 4.8 .

\subsubsection{Turbulence Production and Dissipation}

Similar to the methodology of the field experiments in Chapter 3, turbulence production was calculated as the interaction between Reynolds shear stresses and the gradient of the mean velocity, i.e., $P=\overline{u^{\prime} w^{\prime}} \frac{d \bar{u}}{d z}$. Turbulence dissipation rates, by contrast, were calculated using direct measures of local velocity gradients, rather than through inertial range spectral fitting as previously done. Total viscous dissipation due to turbulent fluctuations is defined as $\epsilon=2 \nu \overline{e_{i j} e_{i j}}$, where $e_{i j}$ is the fluctuating strain-rate tensor. Following the derivation in Fincham et al. (1996), the total dissipation rate for isotropic turbulence can be calculated from 2-dimensional in-plane gradients, which are used to estimate the out-of-plane contribution. Total dissipation is then given by [124]:

$$
\epsilon=\frac{15}{7} \nu\left[2 \overline{\left(\frac{\partial u}{\partial x}\right)^{2}}+2 \overline{\left(\frac{\partial w}{\partial z}\right)^{2}}+\overline{\left(\frac{\partial u}{\partial z}\right)^{2}}+\overline{\left(\frac{\partial w}{\partial x}\right)^{2}}+2 \overline{\left(\frac{\partial u}{\partial z} \frac{\partial w}{\partial x}\right)}\right]
$$

Production and dissipation terms were non-dimensionalized using the model length scale over the cube of the free-stream velocity, $D / u_{\infty}^{3}$. In shear layers, the assumption of isotropic turbulence may no longer be valid, resulting in a much lower contribution to dissipation from the lateral velocity gradients, and as such, the technique applied here may be prone to overestimation [124. However, since each geometry type features a comparably strong shear layer, the error in dissipation should be similar for each model, allowing for valid comparisons between the roughness types.

Plots of turbulence production are shown in figure 4.14 for the low Re regime (left column) and the high Re regime (right column). Given the dependence of turbulence production on $\overline{u^{\prime} w^{\prime}}$, the distributions of $P^{*}$ resemble the pattern of Reynolds stresses in figure 4.13 . As expected, the low-flow regime exhibits very little production along the leading edge, with the exception of regions of vortex ejection from the k-type model; whereas, the high-flow regime shows significant areas of turbulence production along the leading edge for all models due to the large values of $\overline{u^{\prime} w^{\prime}}$. Quantitatively, peak normalized production rates for the 
(a)

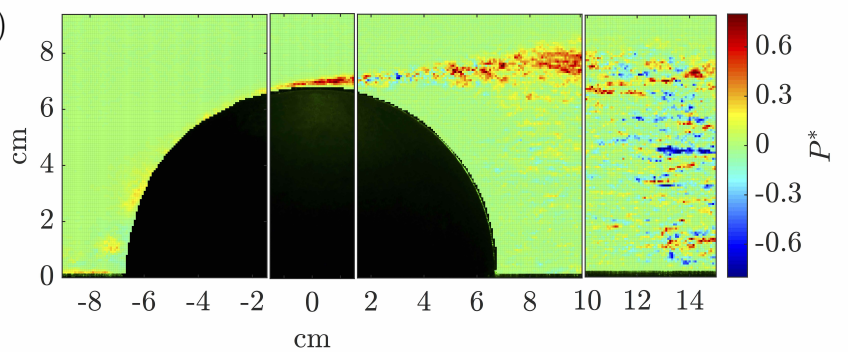

(b)

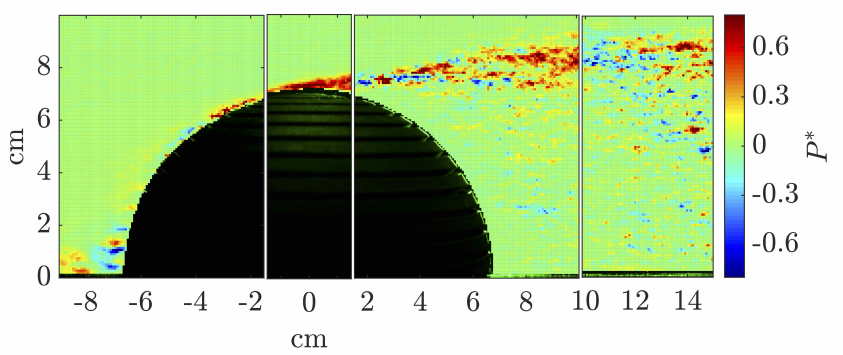

(c)

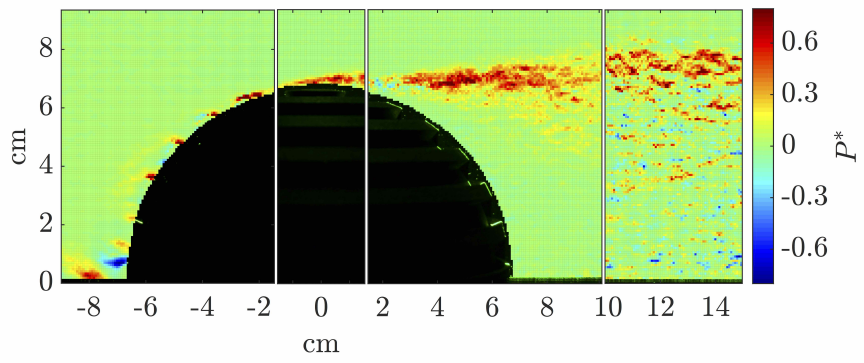

(d)

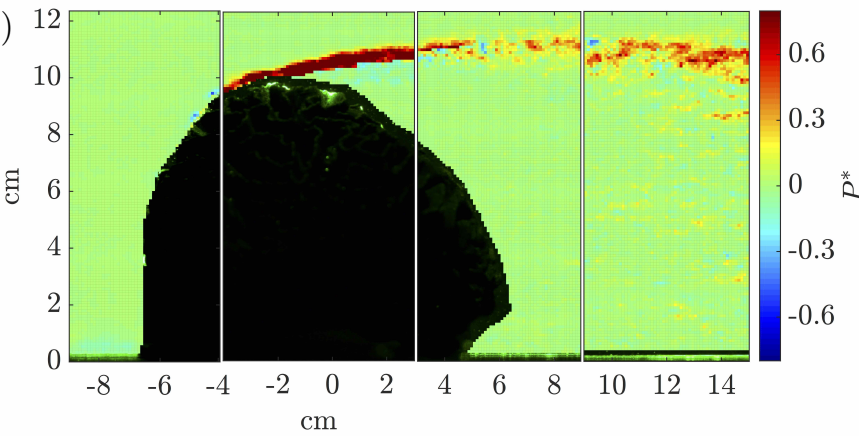

(e)

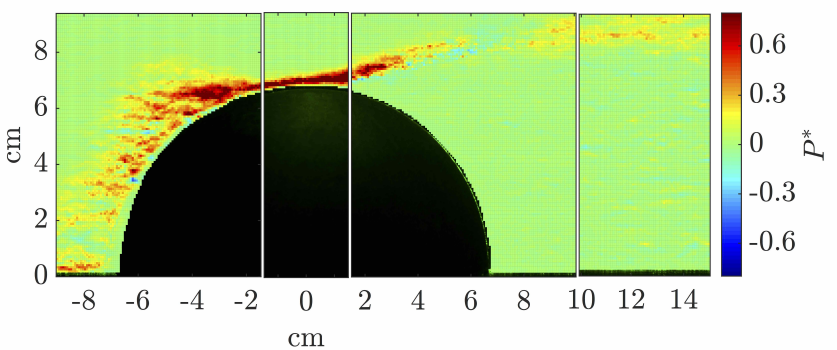

$(f)$

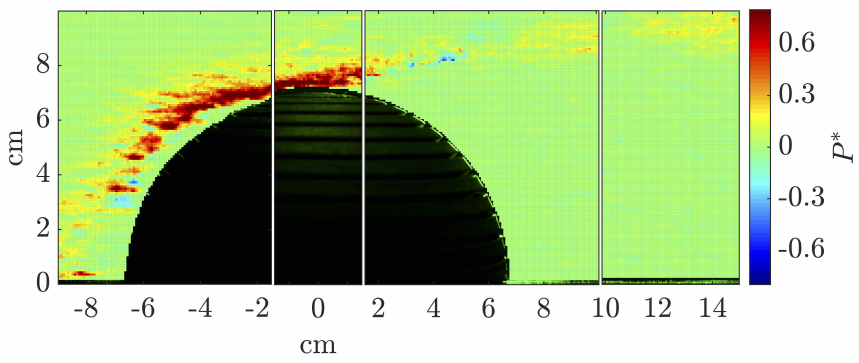

$(g)$

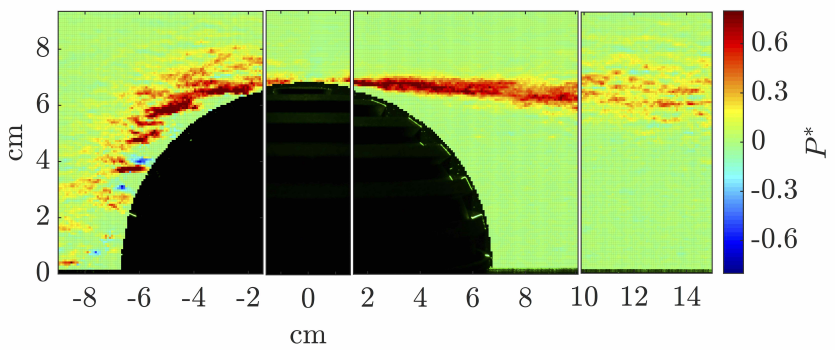

(h)

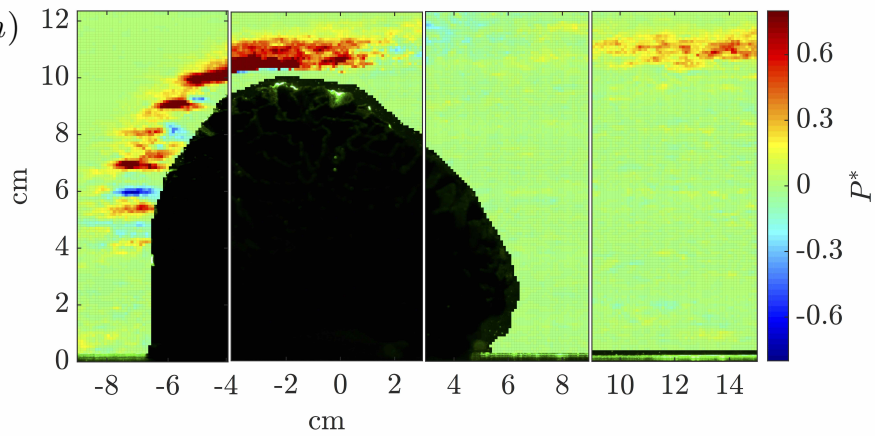

Figure 4.14: Normalized turbulence production for low-flow (left column) and high-flow (right column) regimes. Row 1 is smooth model, row 2 is d-type roughness model, row 3 is k-type roughness model, and row 4 is coral skeleton model. 
d-type, k-type, and coral model are all approximately the same on the leading edge-between 1.8 and 1.9, while the smooth model production is roughly $30 \%$ lower at 1.2 .

All roughness types for both low- and high-flow regimes show strong production levels at the model top, where flow compression is greatest and vertical gradients in mean velocity are largest. Interestingly, for both flow regimes, the very smooth and very rough surface topographies have significantly smaller peak production values in this region compared to the moderate surface roughness of the d-type and coral models 0.81 versus 1.62 , or approximately $50 \%$. Downstream wake regions in the low-flow plots show areas of patchy, strong production, which are negative in areas of flow reversal where $\overline{u^{\prime} w^{\prime}}$ is positive. Quantitatively, peak $P^{*}$ magnitudes in the wake are similar for all roughness types except the coral model, which is roughly $40 \%$ smaller. For the high-flow plots, downstream wakes exhibit low relative magnitudes of turbulence production, likely owing to the high rate of fluid advection, which pushes the shear layer instabilities responsible for eddy formation downstream beyond the PIV window. The exception is the k-type model, whose surface roughness generates vortex shedding itself - evident in the trailing edge wake - in addition to production from shear layer instabilities. This area of high turbulence production in the extended shear layer is nearly $5 \mathrm{x}$ larger than the other models.

Normalized turbulence dissipation rates $D^{*}$ are shown in figure 4.15 for low Re (left column) and high Re (right column) flow regimes. Distribution patterns of $D^{*}$ for the low Re case are very similar for all roughness types, though with increasing areas of dissipation on the leading edge as roughness increases. For the high $R e$ regime, distributions of $D^{*}$ vary significantly with roughness type, with the smooth model showing maximum dissipation at the model top, both moderate roughness models - d-type and coral - showing maximum dissipation at the leading edge, and the roughest k-type model showing maximum dissipation near the top of the trailing edge. Distribution areas and peak magnitudes of $D^{*}$ were significantly smaller for the high $R e$ regime compared to the low $R e$ regime, reflecting the additional contribution of coherent motions present in the low-flow regime.

Quantitatively, for the low $R e$ flow at the leading edge, peak magnitudes of $D^{*}$ are largest for the k-type roughness by approximately $25 \%$ and $75 \%$ compared to the d-type and coral models, respectively (4.1 versus 3.3 and 2.4). The smooth model has negligible dissipation in this region. However, along the model tops at the location of peak shear, this trend is reversed. Here, the d-type and coral models generate $2 \mathrm{x}$ and $3 \mathrm{x}$ higher dissipation rates, respectively, compared to the k-type and smooth models $(1.6$ and 2.3 versus 0.9 and 0.6), which indicates the important role of surface topography on the location of turbulent momentum transfer. On the trailing edge side and in the downstream wake, magnitudes of $D^{*}$ are significantly smaller and comparable across all geometry types. For the high $R e$ flow, peak values of $D^{*}$ are approximately an order of magnitude smaller than the low Re flow and there are few discrepancies in distribution. Leading 
(a)

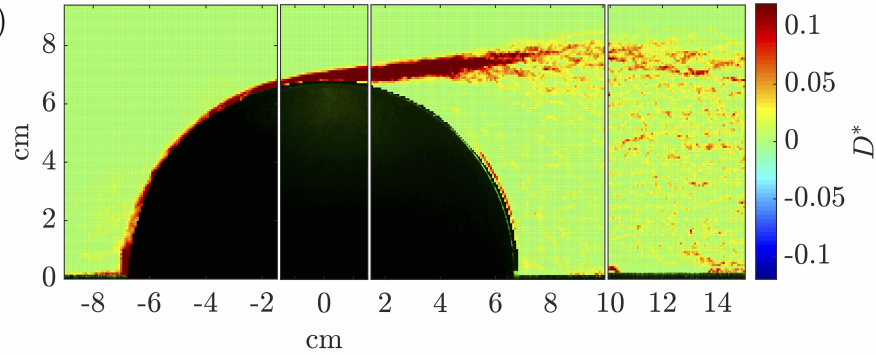

(b)

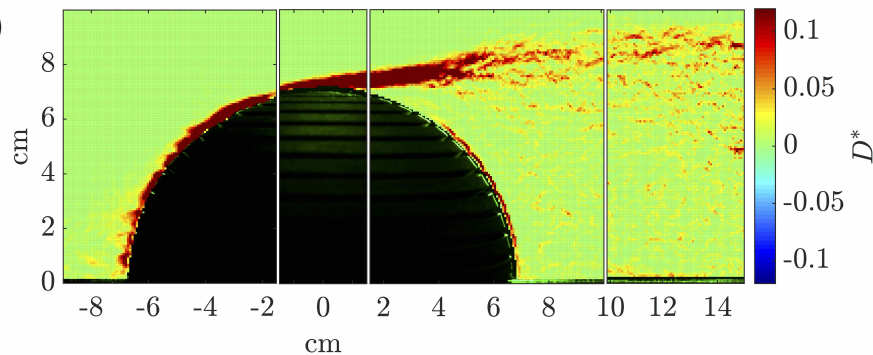

(c)

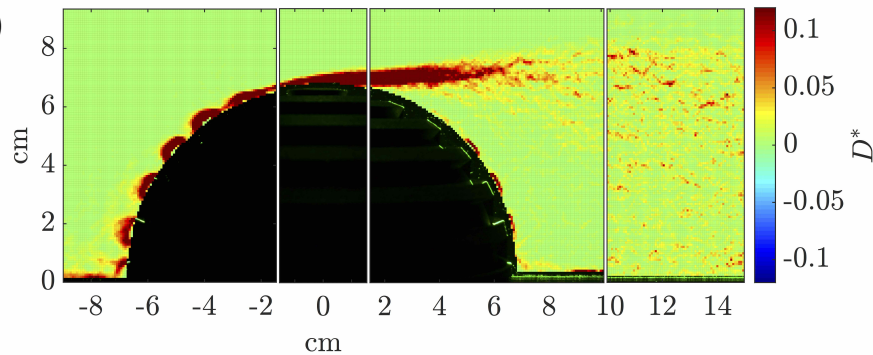

(d)

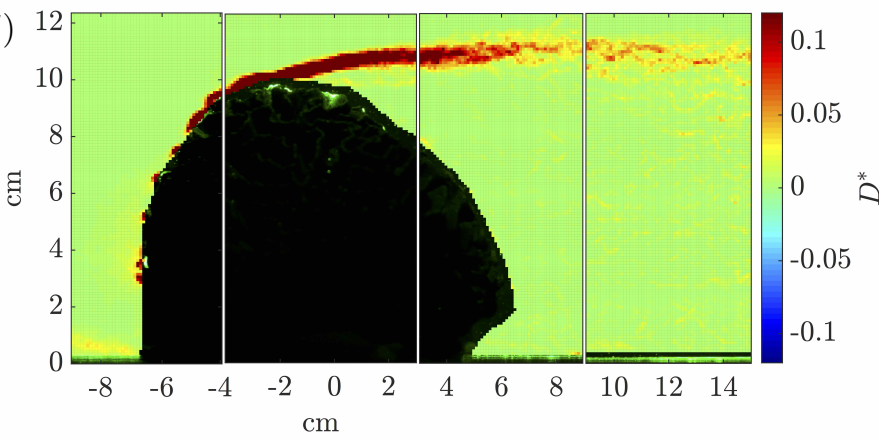

(e)

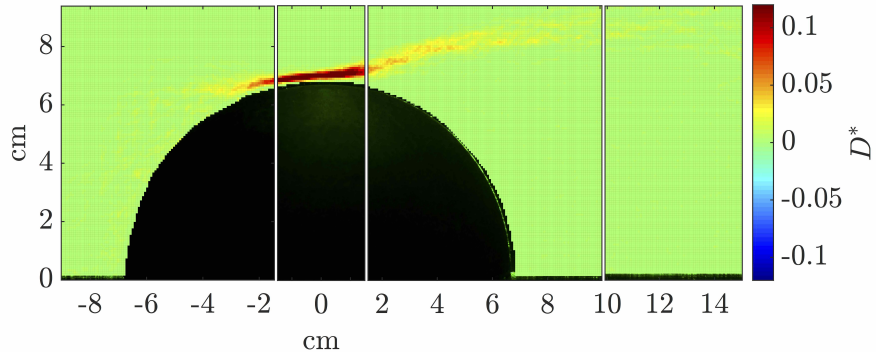

(f)

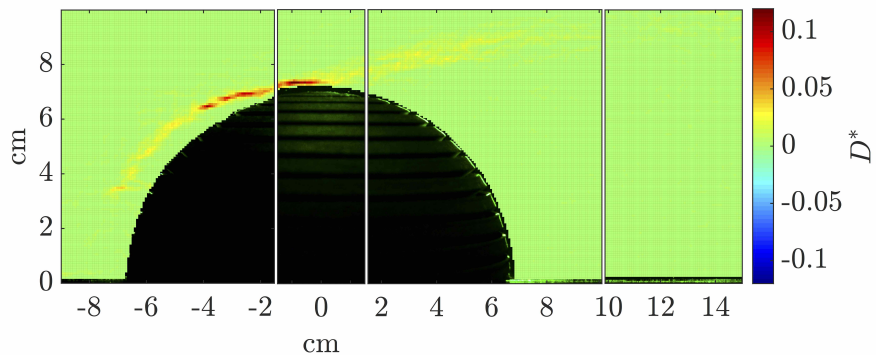

(g)

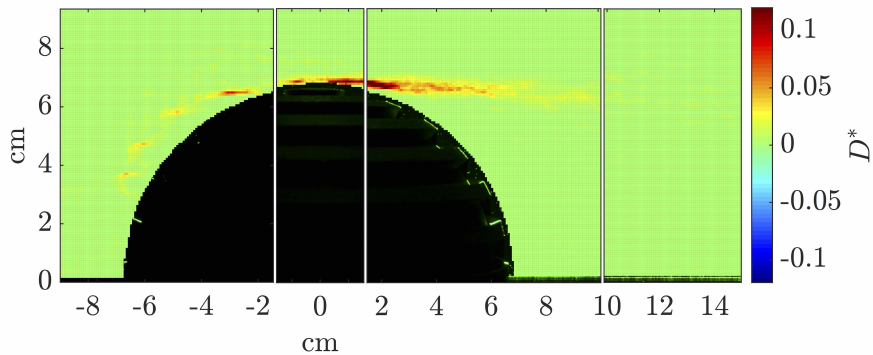

(h)

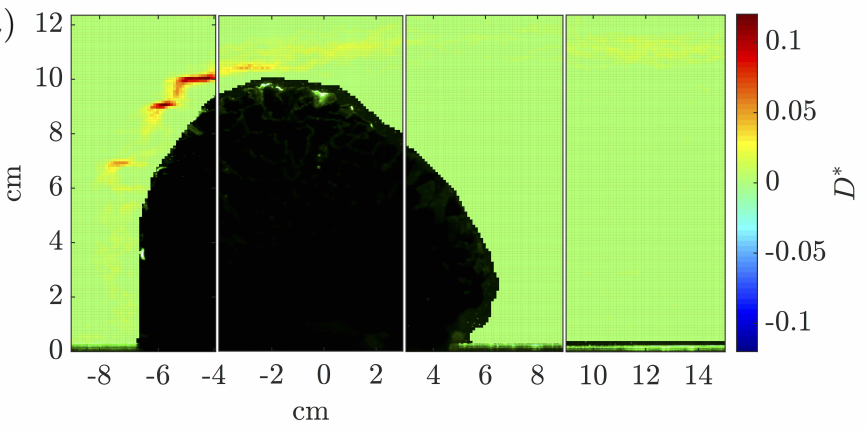

Figure 4.15: Normalized turbulence dissipation for low flow (left) and high flow (right) regimes. 
edge values are all nearly identical (0.09 to 0.13). In contrast, the center shear layer values are largest for the smooth model by $\sim 2 \mathrm{x}(0.32$ versus 0.15$)$. And on the trailing edge, the very rough k-type model exhibits significantly higher values - approximately $5 x$ larger than the other models (0.27 versus 0.05$)$. In the high Re flow, downstream dissipation values are negligible for all geometries $(\sim 0.02)$.

\subsubsection{Joint Probability Distribution Functions}

A joint probability distribution function (p.d.f.) of instantaneous velocity fluctuations provides a convenient tool to examine the anisotropy of Reynolds shear stress and to describe the flux of momentum across the turbulent boundary layer [125]. To construct a joint p.d.f., instantaneous horizontal $u^{\prime}$ and vertical $w^{\prime}$ velocity fluctuations are plotted and contours of like-probability are formed. A quadrant analysis of the probability distribution identifies the relative contributions of turbulent sweep events, i.e., a downward flux of high momentum fluid toward the surface, or burst events, i.e., an outward flux of low momentum fluid away from the surface. Turbulent sweeps are reflected by p.d.f. contours in quadrant IV, where $u^{\prime}>0$ and $w^{\prime}<0$; whereas, turbulent bursts are reflected by p.d.f. contours in quadrant II, where $u^{\prime}<0$ and $w^{\prime}>0$.

Figure 4.16 plots joint probability distribution functions for each model type under low $R e$ flow (top) and high Re flow (bottom). Velocity measurements were taken at a single point within the leading edge boundary layer, located at $x=-4 \mathrm{~cm}$ and at the y-location at which mean horizontal velocity was $10 \%$ of free-stream velocity, i.e., $\bar{u}=0.1 u_{\infty}$. Velocities were rotated into their dominant direction, and
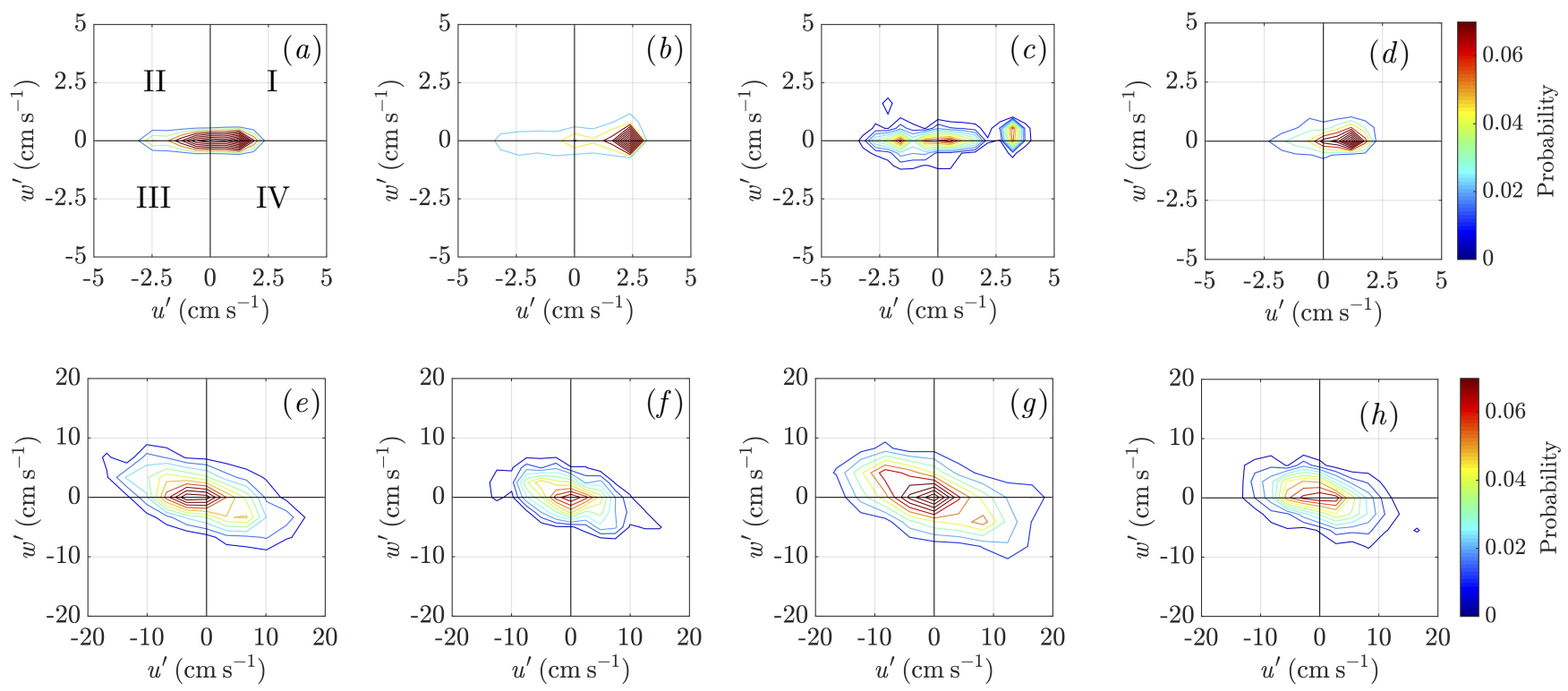

Smooth

D-type

K-type

Coral

Figure 4.16: Joint probability distribution of instantaneous horizontal and vertical velocity fluctuations for low $R e$ regime (top row) and high Re regime (bottom row). 
when compared to the surface-parallel direction, this rotation differed only by $0-15 \%$. This indicates that fluctuations in vertical velocity components reflect a movement of momentum (and mass) either toward $\left(w^{\prime}<0\right)$ or away from $\left(w^{\prime}>0\right)$ the model surface.

For the low Re flow, all four model types show no preferential direction for instantaneous $u^{\prime} w^{\prime}$ cross correlations. The combined contribution of sweep-burst events to total Reynolds stress range from $32 \%$ (for d-type and coral models) to $50 \%$ (for the smooth model), which indicates that the vertical flux of momentum - either the downward injection of fresh fluid or the outward ejection of "dead" fluid — is limited. For the high Re flow, the plots for each model type are remarkably similar, with significant tails of the distribution functions extending predominantly into quadrant II and quadrant IV. The ratio of sweep-toburst events is almost precisely 1:1 for all surface geometries, indicating an equal frequency of high momentum influx and low momentum ejection across the near-surface boundary layer. The combined contribution of sweep-burst events to total Reynolds stress ranges between $67-70 \%$ for all four models, which suggests strong anisotropy of turbulence in the boundary layer and that a majority of Reynolds stresses contribute to surface-normal momentum flux. Joint p.d.f. plots for two other x-axis locations, $x=-2 \mathrm{~cm}$ and $x=-6 \mathrm{~cm}$, were also calculated. These plots (omitted here for clarity) show nearly identical qualitative and quantitative patterns. This indicates that increased sweep-burst events are occurring along the entire leading edge for all model types under high $R e$ flow.

\subsection{Discussion}

The major objective of the experimental work in this chapter is to address the question of how dtype and k-type bar roughness on a wall-mounted hemisphere alter boundary layer turbulence characteristics and downstream wake hydrodynamics. Using PIV data collected at two different Reynolds numbers, mean and turbulence characteristics were calculated and demonstrated the critical impact k-type roughness has compared to d-type or smooth hemispheres on enhancing near-surface shear stress, delaying boundary layer separation, minimizing downstream wake area, and promoting turbulence production. These hydrodynamic effects have direct bearing on mass and thermal fluxes that significantly impact biological processes and may benefit the ecological sustainability of corals. Below, specific results are synthesized, interpreted, and discussed in relation to the current literature and with regard to their biological implications.

\subsubsection{Effect of Reynolds Number}

Reynolds number effects on flows past smooth and rough spheres and hemispheres have long been studied in the literature $85,112,113,117,126$; however, no work has investigated the impact of bar-type 
roughness on hemispheres, which offers an idealized representation of a different class of structures like corals. Though a rigorous parameterization of Reynolds number on a variable such as drag coefficient was not performed here, the results indicate that the low-flow case at $R e=6,750$ and the high-flow case at $R e=20,250$ clearly represent two distinct hydrodynamic regimes. Coherent hairpin structures visible in dye visualization recordings (figure 4.6), reduced polar separation angles (figure 4.9), streamlines showing stable, large-scale vortices in near-wake recirculation zones (figure 4.10), and significantly higher normalized Reynolds stresses in the downstream wake (figure 4.13) due to energetic, large-scale coherent structures - all suggest that the low Re test case represents a subcritical hydrodynamic regime. A subcritical hydrodynamic regime has important implications on momentum transfer and mass/thermal flux in two key regions: 1) the near-surface boundary layer and 2) the downstream wake.

\section{Boundary Layer Structure}

The low Re case also exhibits laminar flow along the leading edge as seen by the absence of Reynolds stresses, turbulence production and dissipation (figures 4.14 and 4.15), and the isotropic joint p.d.f.s in figure 4.16. These data are consistent with the current literature on flow past spheres, which has shown a laminar boundary layer with separation into unsteady vortex shedding up to $R e_{\text {crit }} \sim 10^{5}-10^{6} 127$ for smooth spheres, but as low as $R e_{\text {crit }} \sim 10^{4}$ for rough wall-mounted hemispheres 126. A laminar boundary layer along the model's leading edge without measurable turbulent shear stress means that viscous shear stress dominates contributions to surface shear stress, i.e., $\tau_{s}=\mu \frac{\partial u}{\partial z}$. A recent meta-analysis of field and laboratory experiments has demonstrated a high correlation $\left(r^{2}>0.9\right)$ between mass transfer rates and the root of surface shear stress, $\tau_{s}^{0.4} 128$. This scaling suggests that the $3-4 \mathrm{x}$ higher levels of $\overline{u^{\prime} w^{\prime}}{ }^{*}$ along the leading edge under the high $R e$ regime for all model types would result in a 1.5-1.75x greater mass transfer rate compared to low Re conditions. Enhancement of mass transfer can be achieved through laminar boundary layer thinning at higher $R e$ 93], but the magnitude of enhancement measured here for the high Re case more likely reflects a transition past $R e_{\text {crit }}$, such that mass flux is driven by much more efficient turbulent shear rather than diffusion alone.

\section{Downstream Wake}

The structure and dynamics of the downstream wake are significantly altered by the transition from a subcritical to supercritical flow regime under the high Re test conditions, as suggested by the visibly chaotic wake shedding in dye recordings and streamline plots, the increased polar separation angles, and the reduced wake Reynolds stresses resulting from the loss of coherent structures. Regular shedding of large-scale coherent structures, such as arched vortex tubes resulting from unsteady flow separation, are evident in the low $R e$ 

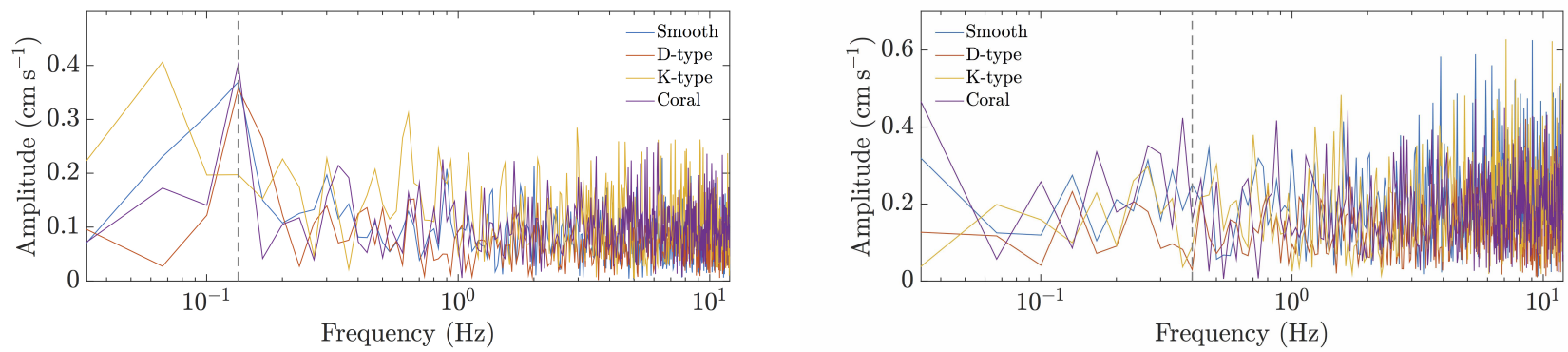

Figure 4.17: Single-sided amplitude spectral density (ASD) of instantaneous vertical velocity fluctuations $w^{\prime}$ for $R e=6,750$ (left) and $R e=20,250$ (right). The dashed vertical line indicates a frequency of $0.13 \mathrm{~Hz}$ and $0.40 \mathrm{~Hz}$ for the low and high $R e$ cases, respectively, which each correspond to a Strouhal number of 0.18.

dye visualizations of figure 4.6, consistent with previous studies 129, 130. Regular shedding of coherent structures occurs at low Reynolds numbers and is indicative of subcritical flow conditions. The periodicity of vortex shedding is characterized by the Strouhal number, a dimensionless ratio of shedding frequency to flow velocity, defined as $S t=\frac{f R}{u_{\infty}}$, where $R$ is the characteristic length scale. For a wall-mounted hemisphere, both model diameter $[129]$ and model radius $[130$ have been used as the length scale to define $S t$, and here, model radius, i.e., $6.75 \mathrm{~cm}$, is used to remain consistent with the literature selected for comparison. While some dependence of $S t$ on Re has been shown [130, for Reynolds numbers comparable to the low-flow condition in this study, previous investigations have found $S t \sim 0.2$, which corresponds to a shedding frequency of $f=0.15 \mathrm{~Hz}$.

Figure 4.17 plots spectral analyses for each model under high and low Re conditions using instantaneous vertical velocity records taken within the centerline shear layer at a downstream position of $x \sim 12 \mathrm{~cm}$. For the low Re regime (left), the smooth, d-type, and coral models all show a clear spectral peak at $f=0.13$, corresponding to $S t=0.18$ and consistent with previous studies, while the spectral peak for the k-type model is shifted to $f=0.07$, or $S t=0.09$. Dye visualization from the k-type model shows a clear disruption of the separating flow and vortex formation due to wake interaction with a downstream roughness element, likely causing the lower shedding frequency (see figure 4.18). Implications of this roughness effect are discussed in the following section. In contrast, the high Re regime shows no dominant shedding frequency for any roughness type, providing further evidence of the loss of coherent structures in the wake at higher flow rates. Further, no dominant frequency for the shear-layer instability resulting from a Kelvin-Helmholtz mechanism (which is distinct from the primary wake shedding instability resulting from a Hopf bifurcation) was detected in the downstream shear layer for either $R e$ regime, likely owing to the relatively low sampling rate $(24 \mathrm{~Hz})$, well below typical Kelvin-Helmholtz frequencies [131]. Coherent structures are an important mechanism for large-scale heat and mass transport [132]; however, the direct enhancement on thermal or scalar flux from an individual bluff body is likely minimal given the limited spatial and temporal extent of interfacial or near- 


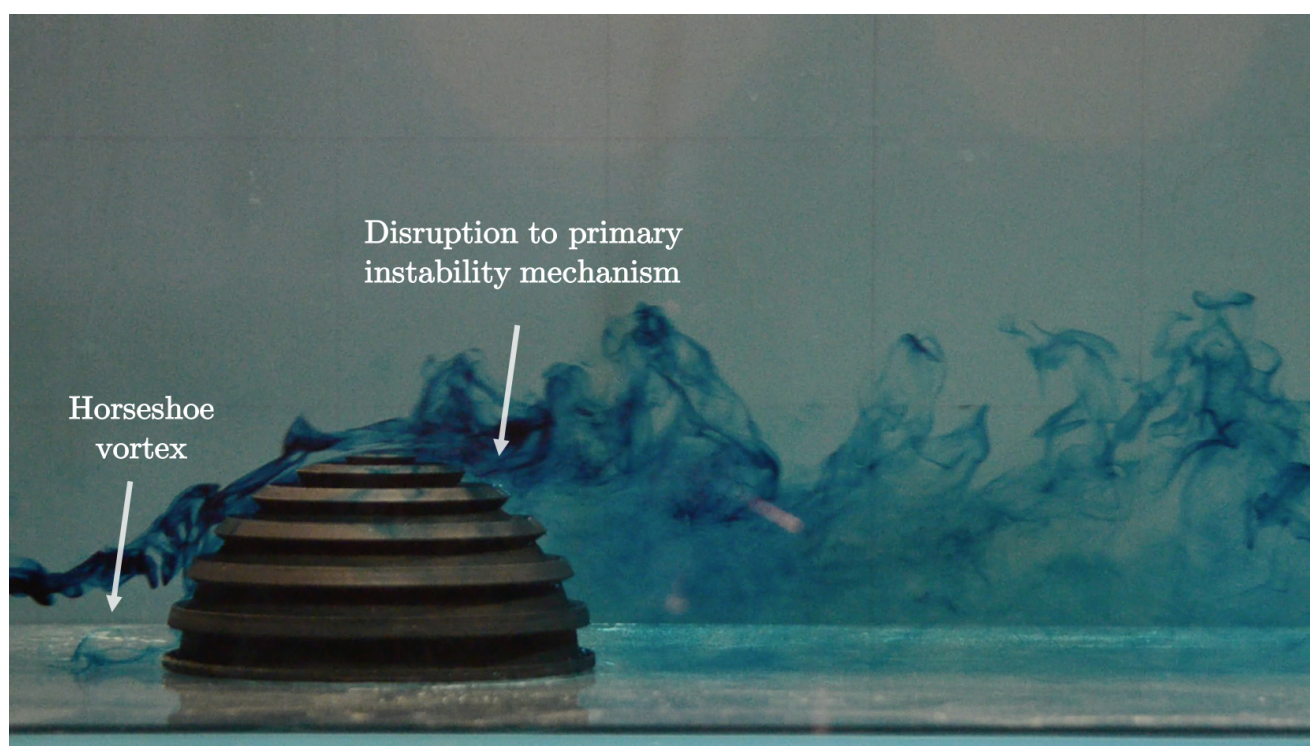

Figure 4.18: Dye visualization of flow past k-type hemisphere at $5 \mathrm{~cm} \mathrm{~s}^{-1}$, i.e., $R e=6,750$. Roughness element on the downstream surface disturbs the flow separation and alters the shedding frequency of large-scale vortex structures. The circle of dye at the leading edge indicates a horseshoe vortex (so-called because of its physical similarity as it surrounds the bluff body) and is typical of boundary layer flow interacting with a wall-mounted bluff body. This is also clearly evident in front of the smooth hemisphere in figure 4.6 .

surface interaction. Rather, coherent structures may contribute more significant impacts in the downstream wake through turbulent transport and mixing of scalars, such as coral larvae, planktonic prey, and waste products [133]. In this way, other reef organisms may be impacted by the coherent structures shed from upstream disturbances, and potentially, under a favorable set of oscillatory conditions, it is possible that the original coral structure itself may be exposed to these coherent motions.

The polar separation angle increases for all four model types under high Re flow, ranging from a $2 \%$ to $20 \%$ increase, which corresponds to a 1-13\% increase, respectively, in the amount of surface area in contact with the highly sheared boundary layer. Areal increases in momentum boundary layer coverage also mean increases in concentration and thermal boundary layer exposure, and thus additional enhancement of mass and heat exchange for the model as a whole. Immediately downstream of the models, the size and strength of the recirculation zone both increased under the high $R e$ test case. Though data was not collected far enough downstream to capture the reattachment point and full streamwise extent of the wake disturbance, it is qualitatively evident from the mean velocity fields in figure 4.8 that the area of recirculation under high $R e$ flow is significantly larger than under low Re flow-an expected result given the much higher rates of fluid advection and also seen in field measurements of wake disturbances behind coral bommies 123. The intensity of passive scalar and thermal mixing in the downstream wake can be parameterized through the use of either eddy viscosity $\nu_{e}$ or mixing length $\ell_{m}$, both of which are inversely proportional to dissipation $\epsilon$ [121]. Thus, larger rates of $\epsilon$ result in smaller mixing lengths and more efficient smearing out of chemical/thermal 
gradients. Dimensional dissipation rates (as opposed to normalized) are 2.5-5.5x higher downstream for the high $R e$ regime compared to the low $R e$ regime, which implies a 60-80\% reduction in size of $\ell_{m}$ and therefore, more intense local mixing.

\section{Implications of Re Effects on Coral Biology}

Since Reynolds number is a function of free-stream velocity and hemisphere diameter, the enhanced flux and mixing benefits derived from higher Re flow may have implications on the selective pressures driving coral size distribution in both low-energy and high-energy flow environments. The low-flow case tested here, for example, is a realistic estimate of velocities found in sheltered habitats or regions with weak tidal currents. Thus, in order to achieve a comparable supercritical Re number of 20,250, the coral diameter would need to be one-third the size of the models tested, potentially offering a competitive advantage to corals that remain small enough to achieve supercritical flow. Such hydrodynamic considerations may help explain the positive correlations between flow rate and coral size shown in surveys of habitat distribution patterns [134], as well as the positive relationship between increased flow rate and differential survival during temperatureinduced bleaching events 135. Additionally, large-scale natural disturbances, such as tropical cyclones or hurricanes, have been shown to preferentially dislodge mid-sized corals 136, which lack the mass of large corals or the low drag forces of small corals needed to prevent destructive lift forces. Though in these cases, the hydrodynamic regime is likely supercritical for all except the largest corals and thus, coral frontal area and mass determine the probability of dislodgment.

The transition from subcritical to supercritical flow over a coral alters the drag coefficients for individual coral structures, which collectively contribute to estimates of total reef-scale form drag used to determine the dissipation of incoming wave and tidal flow, and thus, the degree of shoreline protection [123. Form drag is typically defined as $F_{D}=\frac{1}{2} \rho c_{D} A u_{\infty}^{2}$, where $A$ is the body's frontal area. Thus, for a given flow rate, a larger coral bommie not only obstructs more of the flow-given by $A$-which promotes a larger $F_{D}$, but the bommie diameter may drive the downstream flow into a subcritical regime resulting in a larger $c_{D}$, and also promoting a larger drag force that contributes to an overall increase in reef bottom friction. On the other hand, given a coral of fixed size, if exposed to fast enough flow to create a supercritical $R e$ regime, then $c_{D}$ will be reduced 112,126 , which acts to reduce $F_{D}$; however, the quadratic dependence on $u_{\infty}$ typically outweighs the contribution from $c_{D}$ and thus increases the form drag. In the scenario of fixed body size, the supercritical regime increases $F_{D}$, which, though not measured here, can be inferred from the higher dissipation rates seen in the high $R e$ flow plots in figure 4.15. Rates of turbulence dissipation have been shown to positively correlate $\left(r^{2}>0.86\right)$ to the work done by drag [123], defined as $W=F_{D} U$, so that the increases in $\epsilon$ resulting from high Re flow signal an increase in form drag. The complex interplay 
between coral size, flow speed, and hydrodynamic regime indicate individual and reef-scale effects possess considerable variability across multiple temporal and spatial scales, generating numerous dynamic ecological impacts.

\subsubsection{Effect of Roughness Type}

The results demonstrate a profound impact of surface roughness on the structure and dynamics of boundary layer and wake flow especially for the turbulent high Re regime, but also to a lesser extent for the laminar low Re regime (most evident in increased dissipation rates around individual roughness elements, consistent with previous investigations $137 \mid$ ). Bar-type roughness on a wall has traditionally been categorized by the stability of intra-cavity vortices - with d-type possessing stable vortices, and k-type ejecting vortices into the momentum boundary layer 87]. Given the wall curvature of the hemispheric models studied here, however, it was hypothesized that such a distinction may not hold due to possible flushing effects of incoming flow that penetrates into narrow d-type roughness cavities. The disrupted streamlines at the d-type and coral models' leading edges (figures $4.10 f, h$ ) - similar to those of the k-type model, but to a lesser degree -indicate some degree of outflow from the roughness cavities compared to the orderly streamlines of the smooth model (figure 4.10 e). Other evidence of vortex ejection from d-type and coral roughness is seen in the strong alternating positive and negative patchiness of leading edge vorticity (figures $4.11, h$ ), which is absent over the smooth model. And finally, the near-surface patchiness of very low and very high Reynolds stresses seen over the three rough models (figure $4.13 f-h$ ) indicates the ejection of momentum into the boundary layer, which creates a "buffer" layer, pushing the strongest shear stresses farther from the surface. Though no dominant frequency is seen in a spectral analysis of the surface-normal velocities over any rough model, which would indicate periodicity of ejection, clear evidence from the dye visualization videos show sporadic ejections of dye filaments and support the conclusion that the bluff body curvature reduces the stability of d-type intra-cavity vortices and imparts momentum into the boundary layer flow.

\section{Boundary Layer Structure}

Beyond the stability of intra-cavity vortices, a more quantitative method of surface roughness characterization is the roughness function $\Delta u$, which measures the degree to which surface roughness alters the traditional log-law boundary layer profile by shifting downward the velocity profile relative to a smooth wall. The engineering formulation of the logarithmic law is given by 


$$
\bar{u}=\frac{u_{*}}{\kappa} \ln z+C_{0}-\Delta u,
$$

where $C_{0}$ is a constant equal to $\sim 5.0$ for a smooth wall $[80$. $\Delta u$ can be estimated by plotting velocity profiles for each roughness type at the same location under identical conditions and calculating the average deviation of the logarithmic region from the smooth model. Profiles of streamwise velocity $u$ were created from the $2 \mathrm{D}$ mean velocity plots in figure 4.8 by selecting the leading edge point $x=-4.8 \mathrm{~cm}, z=4.8 \mathrm{~cm}$, which represents a $45^{\circ}$ angle from the model center, and extracting the surface-normal mean velocity record. Figure 4.19 plots on a semi-log scale profiles for each model type under high Re flow. The smooth, d-type, and coral model profiles all collapse to a nearly identical logarithmic layer, while the k-type roughness shows a significant deviation downward, which produces the roughness function, $\Delta u=1.3 \mathrm{~cm} \mathrm{~s}^{-1}$. The roughness function is a measure of a surface's ability to absorb momentum [80], which suggests that the k-type roughness more effectively exchanges mass and thermal properties compared to the smooth or d-type roughness. Direct numerical simulations of flow over roughness have shown that the magnitude of $\Delta u$ depends on the shape and density of the surface topography [38], and that for bar roughness on a flat plate, $\Delta u$ peaks at $w / k \sim 7$, coinciding with a peak in form drag (and negligible skin friction) 138. Empirical heat transfer work demonstrated a similar peak in Nusselt number (a dimensionless ratio of heat transfer coefficient to thermal conductivity) at $w / k \sim 7$ for wedge-shaped ribs, with a dependence on Reynolds number and Prandtl number 139. Both DNS and experimental results found maximum heat transfer coefficients occur at the separation layer reattachment point, approximately 6-8 roughness heights downstream, where shear stress levels are highest [140].

The data collected here also demonstrate a correlation between roughness function and increased Reynolds shear stress levels (and by implication heat and mass transfer rates). Surface-normal profiles of $\overline{u^{\prime} w^{\prime}}$ extracted from figure 4.13 at the same leading edge point as the velocity profiles, i.e., $x=-4.8 \mathrm{~cm}$, $z=4.8 \mathrm{~cm}$, are shown for each model in figure 4.20 . Peak near-surface magnitudes of $\overline{u^{\prime} w^{\prime}}$ occur between 0.5-1.5 cm normal to the surface and are $\sim 33-60 \%$ larger for the k-type roughness compared to the smooth, d-type roughness, and coral skeleton. Higher in the water column, levels of $\overline{u^{\prime} w^{\prime}}$ over the k-type roughness remain elevated by $>50 \%$ for $z>3 \mathrm{~cm}$, indicating enhanced rates of turbulent mixing extending significantly farther outward from the model surface. Though the results indicate that a larger roughness ratio produces greater Reynolds stresses - consistent with previous literature - the ideal spacing ratio of $w / k=7$ found in numerical simulations and empirical studies is not, however, typically seen on corals in nature, likely due to the trade-offs associated with the concomitant loss of surface area. Since total heat (and by analogy, mass) 


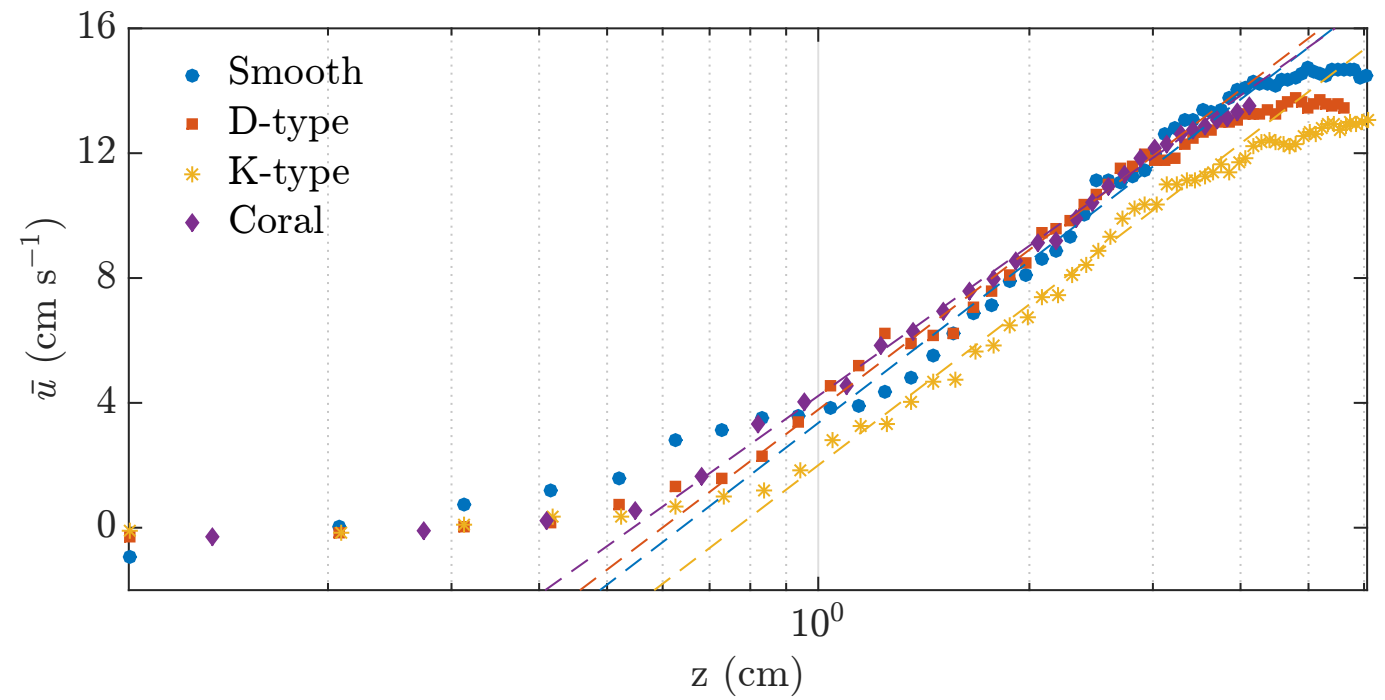

Figure 4.19: Semi-logarithmic profile plots of horizontal velocity normal to model surface along the leading edge boundary layer for each roughness type at $R e=20,250$. The roughness function $\Delta u$ measures $\sim 1.3 \mathrm{cms}^{-1}$ for the k-type roughness.

transfer is given by $q=h A\left(T_{s}-T_{\infty}\right)$, where $h$ is the heat transfer coefficient and $A$ is the surface area, then the increase in $h$ at $w / k \sim 7$ (resulting from greater turbulence generation and bed shear stress) may not be sufficient to overcome the reduction in surface area and may yield a net loss of total heat transfer. Trade-offs between turbulence generation and surface area likely play an important role in the selective pressure driving morphological differences, especially under varying tidal conditions that alter Reynolds number regimes or under wave-driven oscillatory flow conditions in which wave period and orbital excursion lengths become important parameters affecting flow over and within roughness.

\section{Downstream Wake}

The most significant effects of roughness on mean flow quantities in the wake region are seen in the larger polar separation angle and the reduced reattachment length for k-type roughness compared to the smooth and d-type/coral models. For both low Re and high Re regimes, the k-type roughness generates a delay in separation point - approximately $8 \%$ and $32 \%$ larger values of $\Theta_{S}$, respectively - which arise from the injection of momentum by roughness-induced vortices and which have the effect of enhancing the amount of surface exposed to high-shear boundary layer flow, thereby potentially increasing rates of total mass transfer for the body as a whole. An additional direct consequence of a delay in separation is that the wake shear layer is directed downward, rather than upward or level, as seen in figure 4.10. Though the reattachment length is not quantified here because the downstream measurement window is limited, it can be inferred from the streamline plots that the reattachment length behind the k-type model is significantly shortened 

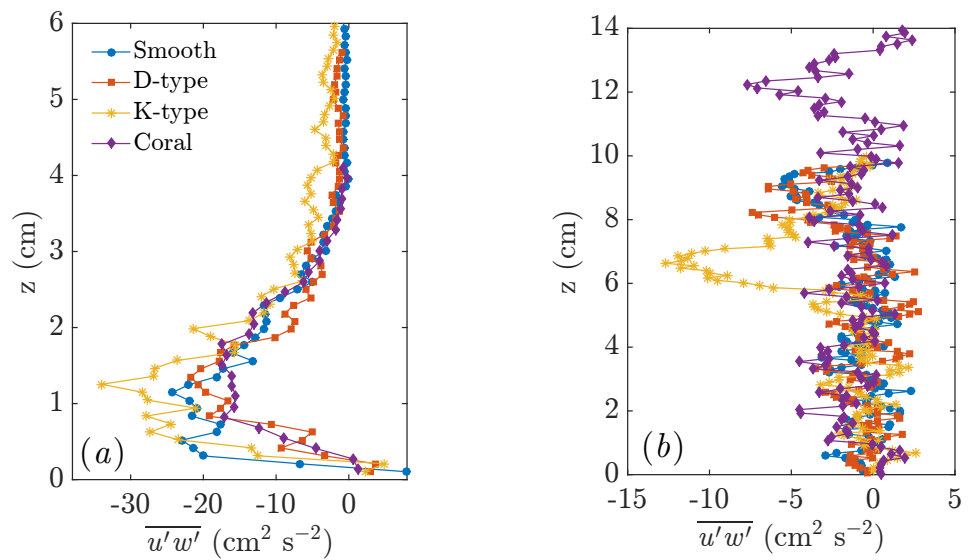

Figure 4.20: Profiles of turbulent Reynolds shear stress at the leading edge at $x=-4.8 \mathrm{~cm}$ (left) and in the downstream wake at $x=9.5 \mathrm{~cm}$ (right) for each roughness type at $R e=20,250$. Peak values for k-type roughness are approximately $50 \%$ and $100 \%$ larger, respectively, compared to smooth and d-type roughness. The much higher vertical location in peak stress for the coral skeleton is a result of the irregular shape of the 3D printed model's base, which elevates the coral skeleton $\sim 3 \mathrm{~cm}$ relative to the other models.

\begin{tabular}{clccc}
\hline & & $\begin{array}{c}\text { Mixing Length } \\
{[\mathrm{cm}]}\end{array}$ & $\begin{array}{c}\text { Eddy Viscosity } \\
{\left[\mathrm{cm}^{2} \mathrm{~s}^{-1}\right]}\end{array}$ & $\begin{array}{c}\text { Shear Layer Thickness } \\
{[\mathrm{cm}]}\end{array}$ \\
\hline \hline$R e=6,750$ & Smooth & $0.30 \pm 0.07$ & $0.29 \pm 0.11$ & 1.57 \\
& D-type & $0.27 \pm 0.05$ & $0.27 \pm 0.06$ & 2.15 \\
& K-type & $0.38 \pm 0.09$ & $0.29 \pm 0.06$ & 2.36 \\
& Coral & $0.23 \pm 0.20$ & $0.17 \pm 0.19$ & 2.04 \\
$R e=20,250$ & & & \\
& Smooth & $0.27 \pm 0.04$ & $0.60 \pm 0.08$ & 1.57 \\
& D-type & $0.35 \pm 0.07$ & $0.78 \pm 0.21$ & 2.44 \\
& K-type & $0.27 \pm 0.03$ & $0.92 \pm 0.09$ & 1.90 \\
& Coral & $0.30 \pm 0.08$ & $0.67 \pm 0.15$ & 1.83 \\
\hline
\end{tabular}

Table 4.2: Estimates (mean \pm one standard deviation) of mixing length $\left(\ell_{m}\right)$, eddy viscosity $\left(\nu_{e}\right)$, and shear layer width $(\delta)$ for each roughness type under low and high $R e$ flows at $x=9.5 \mathrm{~cm}$.

for both high and low flow regimes compared to the smooth and d-type model. This result is consistent with previous experimental findings 126 , which argue that in addition to effects from $\Theta_{S}$, enhanced boundary layer turbulence from surface roughness (seen in the turbulence production plot of Figure 4.14) increases the rate of shear layer spread. The shear layer thickness $\delta$ was defined as the distance between the vertical locations of $90 \%$ and $10 \%$ of free-stream velocity, i.e. $\delta=z\left(u=0.9 u_{\infty}\right)-z\left(u=0.1 u_{\infty}\right)$. Estimates of $\delta$ are given in Table 4.2 and show that the rough surfaces produce a $\sim 17-55 \%$ thicker $\delta$ than the smooth surface, which also implies that vertical mass flux due to turbulent wake mixing occurs over a much larger spatial range for flow behind rough surfaces.

Within the wake shear layer, stress estimates and mixing parameters provide quantitative descriptions of the impact of surface roughness on turbulent hydrodynamics. Profiles of Reynolds shear stress $\overline{u^{\prime} w^{\prime}}$ in the near-wake region at $x=9.5 \mathrm{~cm}$ are shown in Figure 4.20 (right) for the high Re regime. The smooth, 
d-type, and coral models produce similar peak values of $\overline{u^{\prime} w^{\prime}}$ of approximately $6-7 \mathrm{~cm}^{2} \mathrm{~s}^{-2}$, while the peak $\overline{u^{\prime} w^{\prime}}$ for k-type roughness is roughly $2 \mathrm{x}$ as large, or $\sim 13 \mathrm{~cm}^{2} / \mathrm{s}^{2}$. Normalized values (by $u_{\infty}^{2}$ ) compare well with empirical estimates from mixing layer [141] and bluff body wake experiments [142, and these estimates of $\overline{u^{\prime} w^{\prime}}$ can then be used to define mixing parameters such as eddy viscosity $\nu_{t}$ and mixing length $\ell_{m}$. Though the Prandtl mixing-length formulation lacks a valid physical basis for complex three-dimensional turbulence [143, it still provides a useful description of the empirically derived ratio of turbulent shear stresses to the mean velocity gradient, i.e., $\ell_{m}=\left|{\overline{u^{\prime} w^{\prime}}}^{1 / 2} /\right| \partial \bar{u} / \partial z \mid$. Conceptually thought of as the distance over which a parcel of fluid advected through turbulent action gives up its momentum to the surrounding fluid, $\ell_{m}$ was calculated in the midline of the wake shear layer using five measurement bins surrounding $z\left(u=0.5 u_{\infty}\right)$ along the vertical profile $x=9.5 \mathrm{~cm}$. Table 4.2 lists mean \pm one standard deviation mixing length estimates and shows no statistically significant differences exist either between high $R e$ and low $R e$ regimes or between different roughness types within a flow regime. This suggests that the length scale of eddy motion in the wake region is set by the propagation of Kelvin-Helmholtz instabilities originating in the shear layer as determined by the bluff body length scale.

In addition to $\ell_{m}$, the turbulent eddy viscosity $\nu_{e}$ can be used as a quantitative descriptor of the rate at which turbulent action mixes momentum and scalars and is defined as $\nu_{e}=-\overline{u^{\prime} w^{\prime}} /|\partial \bar{u} / \partial z| 61$. Estimates of $\nu_{e}$ are given in Table 4.2 and show that in the low Re regime, roughness type has no effect on $\nu_{e}$, likely due to the dominance of large-scale coherent structures, whose scale is governed by the same bluff body diameter for each model. In the high $R e$ regime, however, $\nu_{e}$ for all roughness types are significantly larger than the low flow estimates, an expected result given that $\nu_{e}$ scales with $R e$. In the chaotic shear layer wake of the high $R e$ flow, though, surface roughness has a significant impact on the magnitude of $\nu_{e}$, with the k-type roughness exhibiting a 50\% larger value than the smooth model and an $18 \%$ and $36 \%$ larger value than the d-type and coral models, respectively (though the variability of the d-type estimate renders the difference not statistically significant). Dimensional arguments dictate that eddy viscosity is proportional to a velocity scale, typically the streamwise fluctuation $u^{\prime}$, and a length scale, typically the mixing length, according to $\nu_{e} \sim u^{\prime} \ell_{m}$. Given that $\ell_{m}$ is identical for all roughness types, this relation implies that the fluctuating velocity scale for the k-type roughness is significantly larger than the other roughness types and thus, turbulent transfer of momentum is occurring more intensely in the k-type wake.

\section{Implications of Roughness Effects on Coral Biology}

At both the leading edge boundary layer and the wake shear layer, the main hydrodynamic results indicate that the d-type roughness and coral model approximate the mean velocity and turbulence characteristics of the smooth model, while the k-type roughness generates significantly more intense turbulent shear, 
mixing, and momentum and scalar transport. At first pass, the most obvious biological implication is that the d-type roughness and the coral skeleton offer a significantly larger total surface area across which interfacial mass flux may occur compared to the smooth surface while maintaining similar turbulence properties. Thus, these surface types more effectively uptake mass-transfer limited nutrients (i.e., conditions in which mass transfer is limited by physical, rather than biological, considerations as is typically the case in coral habitats [22]), remove metabolic waste products, and mitigate excessive thermal heating that may induce bleaching. The assumption, however, is that all surface faces of the d-type or coral model are approximately equally exposed to the same flow conditions. Plaster dissolution experiments of Falter et al. using mm- and cm-scale holes in bluff bodies inferred this to be valid based on a collapse of mass transfer coefficients when scaled by total surface area [37]; however, plaster rectangular bodies with holes more closely resemble porous media or branching corals rather than the hemispheric lobate corals studied here, potentially limiting the usefulness of the comparison. The finding that the d-type and coral models do exhibit some outflow from the roughness cavities due to the model curvature - in contrast to traditional flat-plate d-type bar roughness - does suggest surfaces within roughness cavities may not be entirely isolated from the boundary layer flow and thus total mass transfer may potentially be enhanced compared to a smooth surface.

Larger rates of turbulence production generated by the k-type roughness can have positive impacts on near-surface mixing and shear-driven mass transfer for the bluff body itself as previously discussed, but altered wake hydrodynamics may play a significant role in creating biological niches that other reef organisms can exploit. Within the area of reduced flow downstream of a bluff body, sheltering from strong drag forces may increase the probability of successful settlement of coral larvae [144, and in instances of post-disturbance recovery from bleaching or die-offs, the narrow d-type roughness cavities of the carbonate skeleton may themselves provide suitable attachment sites. Additionally, enhanced vertical fluxes of resources in downstream wakes resulting from turbulence generated by topographic complexity have been shown to promote ecosystem biodiversity in "current-shaded" regions 145. Other large, sessile reef organisms may benefit from free-stream turbulence advected downstream as a result of the wake shear layer, which has been widely shown to enhance heat transfer rates in the engineering literature [146], as well as mass transfer rates in plaster dissolution experiments $[96$ — with a much greater impact on lobate corals like those studied here rather than branching corals. As recirculation length correlates with flow speed [123, the scale and duration of these downstream hydrodynamic niches will vary with current direction and strength on both low frequency diurnal and seasonal scales, but also under high frequency scales of wave-driven oscillatory flow. Finally, measures of drag from an individual coral bommie may scale up to affect total reef-scale drag and bottom friction, which play important roles in wave energy dissipation and shoreline protection from erosion 23. Though drag coefficients were not directly measured, surface roughness has been shown to 
accelerate the transition to a supercritical flow regime, which can be inferred for the models studied here because of the polar separation angle data. In this regime, $c_{D}$ is independent of Reynolds number and $\sim 2 \mathrm{x}$ as large as over a smooth model [85], meaning that for a given supercritical flow speed, the greater turbulence produced by rough coral generates a larger drag force and thus, greater dissipation of flow energy. Reef assemblages are inherently more complex than a collection of smooth or rough hemispheres, and reef-scale drag estimates depend on the nature and density of reef elements, as well as flow conditions with significant effects for unidirectional versus oscillatory flow 63, 123. However, understanding the potential for specific roughness types to alter drag forces may play a useful role in future refinement of reef flow models.

\subsection{Conclusions and Future Work}

This chapter presents the hydrodynamic effects on mean and turbulent flow characteristics due to bar-type roughness on a wall-mounted hemisphere exposed to unidirectional low and high flow conditions. The densely packed roughness topographies - the d-type and the coral skeleton - closely resemble the hydrodynamic conditions of flow past a smooth hemisphere, with the exception of small flow disturbances at the model leading edge. These disturbances suggest that the hemispheric nature of the bluff body allow for some vortex ejection from roughness cavities, which alter mean streamlines and induce spatial patchiness in turbulence quantities like Reynolds shear stress. By contrast, the widely spaced k-type roughness produces a profoundly different hydrodynamic regime at both the leading edge and within the downstream wake. Significant spatial effects along the model surface are seen due to strong vortex ejection from roughness cavities, which inject momentum into the boundary layer causing a large increase in polar separation angle and a much smaller wake recirculation region. The roughness-generated turbulence enhances Reynolds shear stress by $50 \%$ at the leading edge and by $100 \%$ in the wake shear layer, while also accelerating rates of shear mixing as seen by the $\sim 50 \%$ larger eddy viscosity.

It remains an open question as to whether or not the enhanced turbulence parameters resulting from k-type roughness serve to increase total mass transfer for the hemispheric body as a whole. The broad spacing of roughness elements, which function as turbulence generators, also serves to reduce total surface area compared to the d-type roughness. The balance of trade-offs between surface area and mass transfer coefficient may act as a selective pressure on coral morphology and evolutionary development. Numerical simulations, using heat transfer as a proxy for mass transfer, will be undertaken in the following chapter to address this vital question. Additionally, the experimental design of this chapter relied only on unidirectional flow conditions, which account for a minority of coral habitat flow regimes. Far more common are combined wave-current oscillatory flows, which will also be investigated within the numerical models of the final 
dissertation chapter. The experimental results here will be used to validate the numerical results, and taken together, will serve to further our understanding of turbulent boundary layer flow. 


\section{Chapter 5}

\section{Large Eddy Simulations of Heat}

\section{Transfer from Smooth and Rough}

\section{Hemispheres under Unidirectional}

\section{and Oscillatory Flow}

The final chapter of this dissertation presents numerical modeling results of heat transfer from wall-mounted hemispheres with varying surface roughness. Large eddy simulations (LES) of unidirectional and oscillatory flows were performed over the smooth, d-type, k-type, and coral models previously studied in Chapter 4, and the experimental PIV results were used to validate the hydrodynamic outputs of the numerical model, though only for the unidirectional case. Heat transfer simulations were employed within the hydrodynamic model to quantify thermal fluxes, but also, when scaled by the appropriate turbulent Prandtl number, to quantify scalar fluxes. The main objective of this study is to quantify the trade-offs between surface area, turbulence production, and heat transfer resulting from surface roughness (and associated rugosity) under unidirectional and oscillatory flow. Special attention will be paid in describing the largely unstudied question of turbulence characteristics over rough hemispheres in oscillatory flow. The chapter is organized as follows: first, a short review of the major findings on heat/mass transfer and surface roughness will be presented. Next, the LES numerical model and the range of simulation cases will be described. Results of interfacial heat transfer at the model surfaces and vertical heat flux in downstream wakes will then be presented. Finally, the chapter will close with a discussion of the biological implications for corals due to the 
hydrodynamic/heat transfer trade-offs that have been established.

\section{$5.1 \quad$ Introduction}

\subsubsection{Heat transfer fundamentals}

Given that mass transfer between corals and the overlying water column is critical for nearly all biological requirements (e.g., feeding and nutrient capture, reproduction, waste removal, thermal regulation), in this chapter I move beyond a hydrodynamics-only analysis and its implied effect on mass transfer, to a direct investigation of the role of surface roughness on mass transfer. The numerical model implemented in the following section employs heat transfer simulations as a proxy for mass, and thus, I describe here the fundamental heat transfer equations that govern the interfacial flux. Forced external flow over a hemisphere drives heat transfer through convection, i.e., a combination of fluid advection and thermal diffusion. Assuming a constant temperature for all faces of the hemisphere surface, the total heat transfer rate $q$ is defined as 93

$$
q=\bar{h} A_{S}\left(T_{S}-T_{\infty}\right),
$$

where $T_{\infty}$ is the temperature of the overlying water column, $T_{S}$ is the surface temperature, $A_{S}$ is the total surface area, and $\bar{h}$ is the average convection coefficient (i.e., the heat transfer coefficient) given by

the spatial average of local coefficients, $\bar{h}=\frac{1}{A_{S}} \int_{A_{S}} h d A_{S}$. The local heat transfer coefficient depends on the local temperature gradient at the coral surface, the thermal conductivity of the fluid $k$, and the bulk temperature difference according to

$$
h=\frac{-k \partial T /\left.\partial z\right|_{z=0}}{T_{S}-T_{\infty}}
$$

This relation reflects the physical implications of the no-slip boundary condition, which requires that no fluid motion occur at the surface and thus, thermal (and scalar) transfer can only occur through conduction (and diffusion, by analogy). Laminar thinning of the boundary layer at higher Reynolds number or steeper surface gradients following the transition to a turbulent boundary layer would be expected to increase the surface temperature gradient, enhancing the magnitude of $h$ and increasing total heat transfer. The objective of the numerical model is, in general terms, to solve the hydrodynamic conditions throughout the fluid domain, relate the calculated turbulent momentum diffusivity to the thermal diffusivity via the turbulent Prandtl number, and finally, estimate local heat transfer coefficients and by extension, total heat transfer for each hemisphere model. 


\section{Heat-mass transfer analogy}

Regulation of the coral thermal boundary layer against rising sea-surface temperatures and direct solar irradiance is a vital pathway to mitigating coral bleaching and possible die-off. The concerns of ecosystem heat flux management offer sufficient justification to investigate the questions proposed in this chapter, but as an additional benefit, because the functional forms of the heat and mass transfer governing equations are the same, solutions for the heat transfer relationship for a particular geometry are directly analogous to the mass transfer relationship for the same geometry. Thus, the heat transfer simulations undertaken in this chapter also give - after proper scaling - the corresponding mass transfer coefficient, which can be used to address biologically important questions of mass flux. The relationship between the heat transfer coefficient $h$ and the mass transfer coefficient $h_{m}$ is given by 93

$$
\frac{h}{h_{m}}=\frac{k}{D_{A B}\left(\alpha / D_{A B}\right)^{1 / 3}}
$$

where $D_{A B}$ is the scalar diffusion coefficient, which varies with chemical species, and $\alpha$ is the thermal diffusivity. Since $k, \alpha$, and $D_{A B}$ are all known, intrinsic fluid or chemical properties, theses values can be used to estimate the mass transfer coefficient $h_{m}$ once the heat transfer coefficient $h$ has been modeled. A specific example demonstrating this conversion for a relevant chemical species will be given in the discussion below.

\subsubsection{Convection heat transfer correlations}

Convection heat transfer correlations, which relate hydrodynamic flow parameters to heat transfer parameters, have long been developed in the engineering literature and are typically given in terms of the Nusselt number $N u$, the dimensionless ratio of total heat transfer to conductive heat transfer, i.e., $N u=\frac{h L}{k}$, where $L$ is the appropriate length scale. (The mass transfer analogue, called the Sherwood number $S h$, employs the mass transfer coefficient $h_{m}$ and scalar diffusivity $D_{A B}$ in place of $h$ and $k$, respectively.) Since $k$ is a known fluid property and total heat transfer $q$ can be measured directly, $N u$ is a convenient relation for use in determining the heat transfer coefficient $h$. Larger values of $N u$ indicate greater convection transfer. For laminar flow over a flat plate, analytic solutions to the governing momentum equations are well-known (see any standard introductory heat transfer text, e.g. Incorpera and DeWitt [93]) and give the following one-dimensional local convection heat transfer relation

$$
N u_{x}=0.332 \operatorname{Re}_{x}^{1 / 2} \operatorname{Pr}^{1 / 3}
$$


which is valid for $\operatorname{Pr}>0.6$. (Prandtl number is a dimensionless fluid property relating viscous diffusion to thermal diffusion, $\operatorname{Pr}=\nu / \alpha$ ). This relation suggests that for a given fluid, i.e., a fixed Prandtl number, the heat transfer coefficient scales directly with Reynolds number. For turbulent flow over a flat plate, analytical solutions are not available, rather empirical approximations of friction coefficients and boundary layer thickness have been derived to yield a local convection correlation given by

$$
N u_{x}=0.0296 \operatorname{Re}_{x}^{4 / 5} \operatorname{Pr}^{1 / 3}
$$

also valid for $\operatorname{Pr}>0.6$. In contrast to equation 5.4. $N u$ exhibits a stronger dependence on Reynolds number for a turbulent boundary layer, and the enhanced mixing due to turbulent fluctuations generates a larger boundary layer and larger heat transfer coefficient.

\subsubsection{Rough-wall heat transfer}

The question of interest in this chapter, however, is how does surface roughness, rather than a simple flat plate, alter convection coefficients and rates of total heat transfer. Even without the complication of a turbulent boundary layer, analytical solutions of momentum equations over roughness are not available, and solutions require either empirical correlations or direct numerical simulations. Pioneering experimental work on heat transfer in smooth and rough pipe flow was undertaken by Dipprey and Sabersky (1963), in which the authors show that "closely packed" spherical roughness elements, analogous to the sand grain roughness of Nikuradse, enhanced friction coefficients and heat transfer coefficients by $2-3 \mathrm{x}$ compared to smooth tubes across a range of Reynolds numbers, peaking near the transition region 147 11 Subsequent experimental studies showed that variations in the surface geometry play a role in determining heat transfer correlations, having demonstrated dependencies for both sand grain-like size and density 148 and for two-dimensional rib roughness [140]. The results suggest that a universal correlation based on roughness equivalence can not successfully collapse heat transfer rates, but instead a heat-momentum analogy based on law-of-the-wall similarity profiles (i.e., similar rough-wall velocity shifts rather than similar roughness sizes) gives more accurate correlations. More recent direct numerical simulations, however, have shown that even this may be an insufficient universal correlation, demonstrating an approximately $50 \%$ greater Nusselt number for 2-D ribs compared to sand grain roughness with an equivalent roughness function $\Delta u[149$. Rather, an additional term accounting for the wetted surface ratio of the roughness more accurately, though not fully, captures

\footnotetext{
${ }^{1}$ The augmentation in heat transfer results from an increase in skin friction, which for industrial applications poses a trade-off in convection pumping demands versus heat transfer, but for natural convection in the environment, no such challenges exist. Rather, in the near-shore environment, the enhanced bottom friction from cumulative small-scale to reef-scale topographic roughness promotes wave energy dissipation and shoreline protection.
} 
rough-wall heat transfer correlation 150. Differences likely remain owing to the nature of the turbulence generated by various roughness types, with large-scale turbulent structures produced by repeated ribs more strongly enhancing near-wall mixing and promoting larger rates of heat transfer.

For two-dimensional repeated rib roughness, the question raised by the hydrodynamic results of the previous chapter is: how do trade-offs in surface area and roughness ratio affect total heat transfer? In particular, the larger width-to-height ratio of the k-type surface was shown to generate greater turbulence and mixing, but at the expense of a lower total surface area available for heat and mass transfer. For flatplate roughness, the optimization question was addressed through direct numerical simulations of the $w / k$ parameter space, finding that the rough-wall velocity shift and average Nusselt number both peak at $w / k \sim 7$ 138, 151. Drag forces and large-scale turbulent motions are maximized at these $w / k$ values, which suggests that the heat transfer enhancement due to turbulence outweighs contributions from simple surface area gains. The question remains, however, as to whether or not bar roughness on a hemisphere - rather than a flat plate - optimizes heat transfer in the same way, especially for oscillatory flows.

\subsubsection{Heat transfer for hemispheres}

Local rates of heat transfer around the surface of a hemisphere - similar to cylinders and spheresvary significantly with coordinate angle, reflecting the distribution of local skin friction and post-transition turbulent mixing [93. For subcritical, low Re flows, local $N u_{\theta}$ is largest near the leading edge stagnation point, decreases to a minimum at the separation point, and rises along the trailing edge. In contrast, for supercritical, high $R e$ flow, local $N u_{\theta}$ peaks sharply within the transition region, before reaching a minimum at the separation point, then rises again due to wake mixing. Though spatial variations in heat and mass transfer have important biological implications, often the more important parameter is the overall average transfer rate $\overline{N u}$. For a smooth sphere, elaborate empirical heat transfer correlations have been proposed

$$
\overline{N u}=2+\left(0.4 \operatorname{Re}^{1 / 2}+0.06 \operatorname{Re}^{2 / 3}\right) \operatorname{Pr}^{2 / 5}\left(\frac{\mu}{\mu_{s}}\right)^{1 / 4},
$$

where $\mu$ is the viscosity at the bulk fluid temperature and $\mu_{s}$ is the viscosity at the average surface temperature 152. The spherical correlation equation has been shown to be applicable across a range of Prandtl and Reynolds numbers, though significant deviations of up to $20 \%$ remain. Very little experimental work has been done on rough sphere heat transfer directly, but it is expected that hydrodynamic findings showing larger surface roughness increases drag coefficients in supercritical flow 85 would act to increase skin friction and increase $\overline{N u}$. 


\subsubsection{Mass transfer for oscillatory flow}

Finally, the question of how oscillatory flow affects heat transfer rates for both smooth and rough flat plates and spheres is largely unstudied in the engineering literature. A number of mass transfer experiments based on gypsum dissolution, however, have been performed in the environmental science literature. Oscillatory flow over cylinders within an idealized canopy array was shown to drive mass transfer rates up to $3 \mathrm{x}$ higher than for comparable unidirectional flows, and exhibited a strong dependence on canopy element spacing, such that denser spacing drove faster in-canopy flow and mass transfer 153. Similar enhancements of up to $3 \mathrm{x}$ were demonstrated for gypsum models of branching corals, also showing enhancement for a reduction in branch spacing as well as for increasing wave frequency 64. Significantly smaller rates of mass transfer enhancement, only up to $60 \%$, were found for rectangular prisms with roughness features exposed to oscillatory flow 37. The mass transfer rates also scaled linearly with surface area, seemingly in contrast to the bulk of rough-wall heat transfer research, but the roughnesses employed were all narrowly spaced d-type roughness. Thus, the large-scale motion and enhanced turbulent mixing generated by k-type roughness was likely not present and its potential transfer effects were omitted.

\subsubsection{Research questions}

The major objective of this chapter is to extend the experimental results of the preceding chapter through numerical simulations, and in doing so, to address two significant gaps in the literature: 1) oscillatory flow past a hemisphere and 2) heat transfer for a rough hemisphere. These considerations lead to the following specific research questions:

\section{What are the hydrodynamic characteristics of the turbulent boundary layer and near-field wake} for smooth and rough hemispheres under oscillatory flow?

To address this question, large eddy simulation (LES) numerical models will be developed, and validated using the unidirectional experimental results of Chapter 4, in order to characterize the impact of surface roughness and oscillatory flow on turbulence characteristics.

2. How does surface roughness affect heat transfer for a hemisphere in unidirectional and oscillatory flows, and what are the implications for trade-offs among surface roughness, surface area, turbulence generation, and heat transfer coefficients?

A heat transfer model will be coupled to the hydrodynamic LES numerical model to determine thermal flux characteristics over the various hemispheres, and these results will be used to evaluate the balance between 
roughness-generated turbulence/heat transfer and the corresponding surface areas available for thermal and scalar flux.

\subsection{Formulation of the Numerical Model}

\subsubsection{Domain setup and boundary conditions}

The geometry of the model domain was designed to replicate the experimental test conditions used in the PIV water flume experiments of Chapter 4. The $x, y$, and $z$ axes are aligned with the streamwise, cross-stream, and wall-normal directions, respectively, and the corresponding velocities are given by $u, v$, and $w$. The fluid domain comprises a rectangular box measuring $90 \mathrm{~cm} \mathrm{x} 38 \mathrm{~cm} \mathrm{x} 40 \mathrm{~cm}$ in the $x, y$, and $z$ coordinates, respectively. The digital STL files for each of the four surface-type model types were imported into the fluid domain and positioned centered on the floor of the box, i.e., such that the center of each hemisphere aligned to $x=45 \mathrm{~cm}, y=19 \mathrm{~cm}$, and $z=0 \mathrm{~cm}$. Each model was then Boolean subtracted from the fluid domain to create the appropriate surface geometry boundary condition. Figure 5.1 (left) illustrates the resulting geometry of the fluid domain, using the $w / k=2.0$ model as an example. Figure 5.2 shows close-up images for each of the four hemisphere geometries as seen from the near-wall in the direction of the positive $y$ axis.
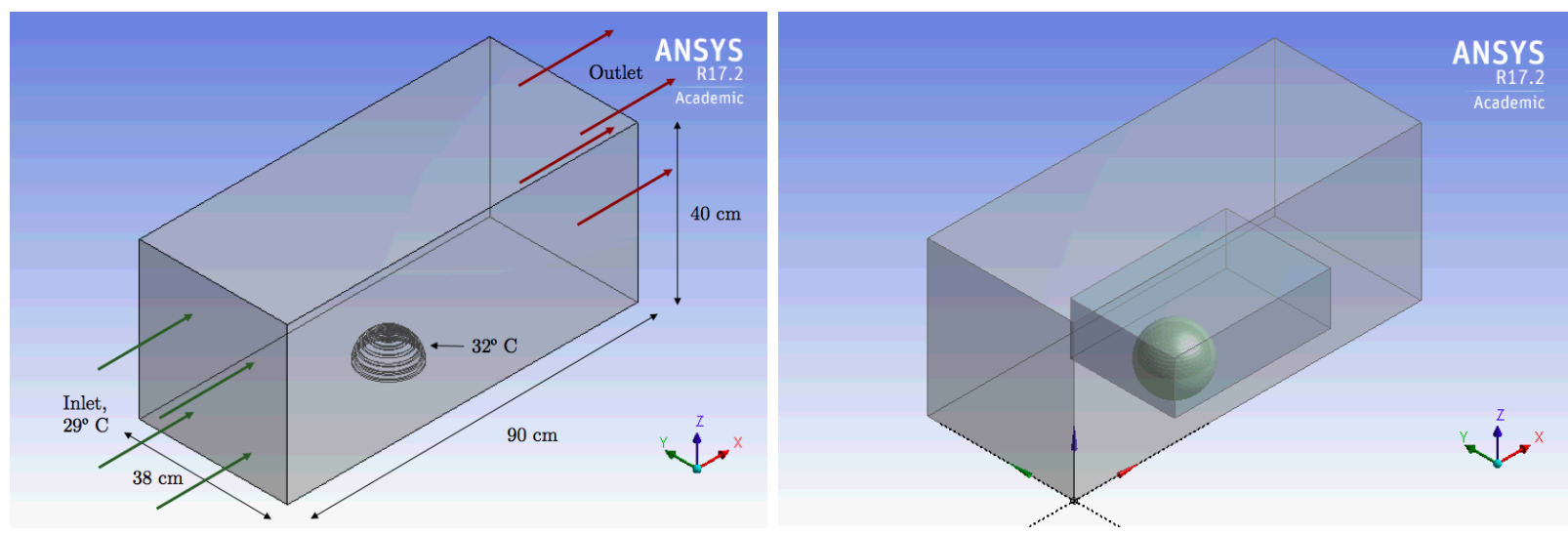

Figure 5.1: Left. Fluid model domain with $w / k=2.0$ hemisphere as example. Right. Model domain showing placement of "high-resolution" box (for unidirectional case) and "super high-resolution" concentric sphere used for targeted grid discretization to improve simulation accuracy in flow regions of greatest interest.

\begin{tabular}{lcccc}
\hline Parameter & Smooth & $w / k=0.5$ & $w / k=2.0$ & Coral \\
\hline \hline Total surface area, TSA $\left(\mathrm{m}^{2}\right)$ & 0.029 & 0.062 & 0.044 & 0.079 \\
Projected planar area, PPA $\left(\mathrm{m}^{2}\right)$ & 0.014 & 0.014 & 0.014 & 0.014 \\
Rugosity, $R u=$ TSA/PPA $(-)$ & 2.00 & 4.31 & 3.09 & 5.56 \\
\hline
\end{tabular}

Table 5.1: Description of surface parameters for model geometries. 

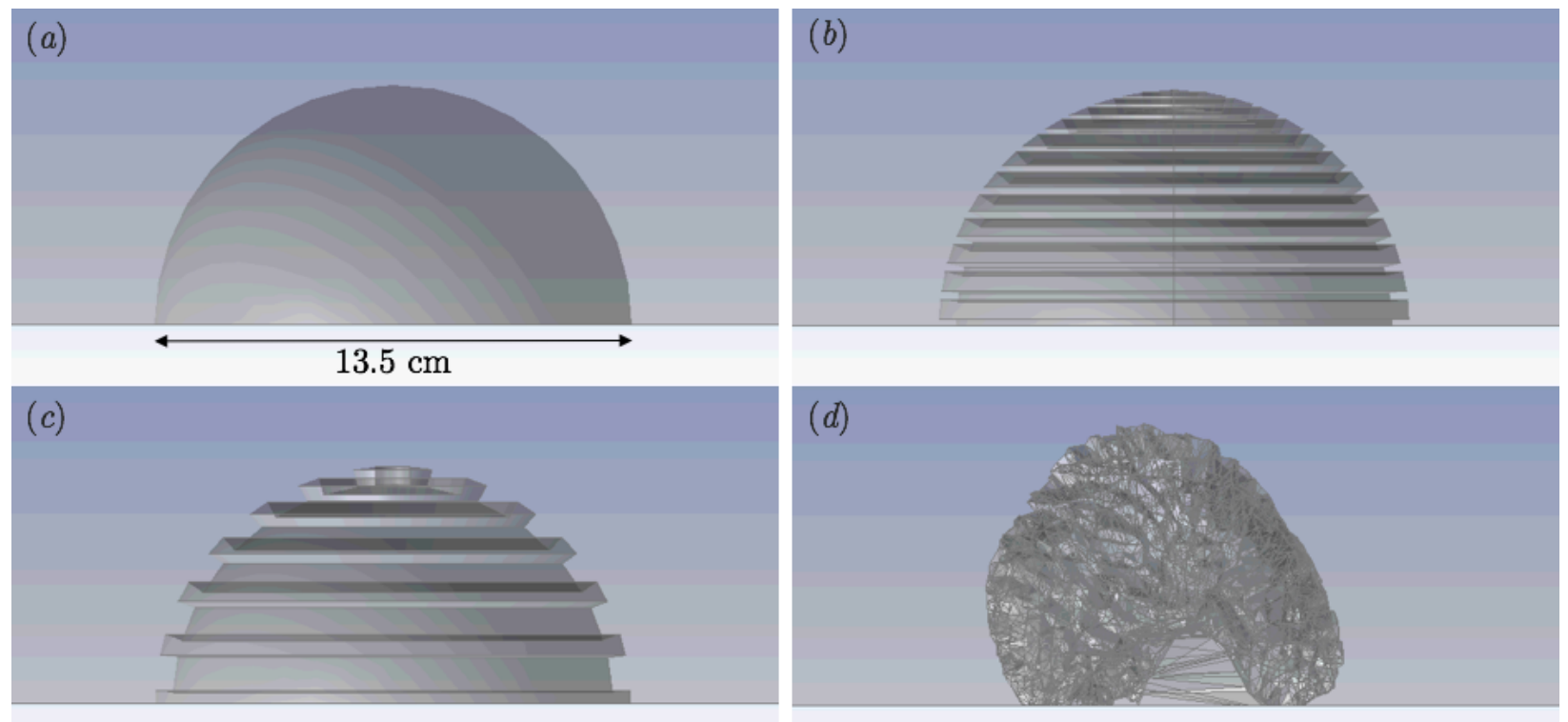

Figure 5.2: Close-up view in $x$ - $z$ plane of the four model geometries: $(a)$ smooth hemisphere, $(b)$ d-type hemisphere with $w / k=0.5,(c)$ k-type hemisphere with $w / k=2.0$, and $(d)$ coral skeleton.

For the near-wall, far-wall, and bottom boundaries, an adiabatic no-slip wall condition is applied. The top boundary is assigned an adiabatic free-slip wall condition, and the hemisphere surfaces for each model are assigned a no-slip condition and a fixed surface temperature of $32^{\circ} \mathrm{C}$. Flow is driven at the inlet wall by a prescribed streamwise $u$ velocity, depending on the test case, and with an inlet temperature of $29^{\circ} \mathrm{C}$ for all cases. For each model geometry, four flow conditions were investigated: 1) unidirectional low flow, $u(t)=5 \mathrm{~cm} \mathrm{~s}^{-1} ; 2$.) unidirectional high flow, $\left.u(t)=15 \mathrm{~cm} \mathrm{~s}^{-1} ; 3\right)$ oscillatory low flow, $u(t)=7.07 \cos (\omega t)$; and 4) oscillatory high flow, $u(t)=21.21 \cos (\omega t)$. For the oscillatory conditions, $\omega=2 \pi / T$ is the radian frequency, where $T$ is the wave period set to $5 \mathrm{~s}$, a typical timescale for wave conditions found in coral habitats. The $u_{\max }$ values for the oscillatory cases, i.e., $u_{\max }=7.07$ and $u_{\max }=21.21$, were selected in order to give a root-mean-squared (RMS) value equal to the unidirectional low- and high-flow velocities, respectively. By formulating the oscillatory conditions in this way, the total fluid motion past the hemisphere over a given amount of time is equivalent for the unidirectional and oscillatory cases. Additionally, equivalent RMS velocities for unidirectional and oscillatory flows allow for appropriate Reynolds number matching between the two regimes, where $R e=\left(u_{r m s} D\right) / \nu$, based on the hemisphere diameter $D$. Table 5.1 lists the total surface area and corresponding rugosity value for each roughness type, and table 5.2 (below) lists the complete set of hemisphere roughnesses, flow conditions, and corresponding Reynolds numbers used for the simulations. 


\subsubsection{Large eddy simulation fundamentals}

To investigate the model problem developed in the preceding section, large eddy simulations (LES) were employed. LES represents a compromise between the prohibitively expensive computational cost of direct numerical simulations (DNS), which solve all scales of turbulent motion to the Kolmogorov scale, and the less accurate, less expensive Reynolds-stress models, such as the Reynolds-Averaged Navier Stokes (RANS), which model all scales of turbulent motion. To achieve this compromise, LES directly computes the largescale motions - at the resolution of the domain grid spacing - and uses an eddy viscosity model to represent the small-scale motions between grid points. In this way, LES avoids calculating the most computationally expensive small-scale turbulent motions, which typically possess a more universal character compared to the geometry-dependent large-scale motions. LES is particularly useful for high Reynolds number flows over bluff bodies, in which the high Reynolds number renders DNS impractical, and the bluff body produces significant unsteadiness and vortex shedding, which RANS models would fail to capture.

In practice, the LES procedure involves four steps to calculate the turbulent flow field, which can be found in any standard computational fluid dynamics text, e.g. Pope (2000) 121. First, the instantaneous three-dimensional velocity field $\boldsymbol{u}(\boldsymbol{x}, t)$ is decomposed into a filtered component $\overline{\boldsymbol{u}}(\boldsymbol{x}, t)$, i.e., resolved at the grid points, and a residual component $\boldsymbol{u}^{\prime}(\boldsymbol{x}, t)$, i.e., the remaining subgrid-scale (SGS) velocity component. Second, in order to describe the evolution of the filtered velocity field, the Navier-Stokes equations are used to derive governing equations, which include a residual stress tensor that accounts for subgrid-scale motions. Third, the governing questions are closed through the use of an appropriate eddy viscosity model to represent the residual stress tensor. Finally, the equations describing the filtered velocity field $\overline{\boldsymbol{u}}(\boldsymbol{x}, t)$ are solved numerically to produce an approximation of the large-scale turbulent motions at the resolution of the model grid, while the SGS eddy viscosity model approximates the flow field at the residual scale.

\subsubsection{LES governing equations and subgrid-scale model}

The LES governing equations are obtained by applying a low-pass filter, typically in the form of an explicit box or Gaussian filter, to the time-dependent Navier-Stokes equations. In this work, however, the commercially available ANSYS CFX 17.2 package was used for LES model formulation and solving. As such, the filtering procedure was achieved implicitly through the discretization of the model spatial domain into finite control volumes, according to the general formulation

$$
\bar{\Phi}(\boldsymbol{x})=\frac{1}{V} \int_{V} \Phi\left(\boldsymbol{x}^{\prime}\right) d \boldsymbol{x}^{\prime}, \boldsymbol{x}^{\prime} \in V,
$$


where $\Phi$ is the relevant variable (and the overbar indicates a filtered variable), $V$ is the control volume, and $\boldsymbol{x}^{\prime}$ indicates a coordinate location within the control volume, i.e., a residual, or subgrid-scale, coordinate. (The implied filter function being $G\left(\boldsymbol{x} ; \boldsymbol{x}^{\prime}\right)=1 / V$, for $\boldsymbol{x}^{\prime} \in V$; and $=0$, otherwise.) Employing the Boussinesq approximation, the resulting filtered continuity and momentum equations are given, respectively, in tensor notation as

$$
\begin{gathered}
\frac{\partial \bar{u}_{j}}{\partial x_{j}}=0 \\
\frac{\partial \bar{u}_{i}}{\partial t}+\frac{\partial}{\partial x_{j}}\left(\bar{u}_{i} \bar{u}_{j}\right)=-\frac{1}{\rho} \frac{\partial \bar{p}}{\partial x_{i}}+\nu\left(\frac{\partial^{2} \bar{u}_{i}}{\partial x_{j} \partial x_{j}}\right)-\frac{\partial \tau_{i j}}{\partial x_{j}},
\end{gathered}
$$

where $\tau_{i j}$ is the subgrid-scale stress tensor defined by $\tau_{i j}=\overline{u_{i} u_{j}}-\bar{u}_{i} \bar{u}_{j}$, and which requires modeling for closure.

In order to relate the subgrid-scale stresses to the resolved large-scale strain rate tensor $\bar{S}_{i j}$, a SGS eddy viscosity $\nu_{T}$ is employed, according to

$$
\tau_{i j}=-2 \nu_{T} \bar{S}_{i j}=-\nu_{T}\left(\frac{\partial \bar{u}_{i}}{\partial x_{j}}+\frac{\partial \bar{u}_{j}}{\partial x_{i}}\right)
$$

ANSYS CFX provides several options to model $\nu_{T}$, and for the simulations performed here, the Wall-Adapted Local Eddy-viscosity (WALE) model of Nicoud and Ducros (1999) was selected [154]. The LES-WALE model utilizes both the strain rate and rotation rate of the resolved turbulence to estimate $\nu_{T}$ and achieves proper $y^{3}$ scaling in near-wall regions, without requiring the time-intensive secondary filtering of typical dynamic models. The WALE model formulation of $\nu_{T}$ is given by

$$
\nu_{T}=\left(C_{w} \Delta\right)^{2} \frac{\left(S_{i j}^{d} S_{i j}^{d}\right)^{3 / 2}}{\left(\bar{S}_{i j} \bar{S}_{i j}\right)^{5 / 2}+\left(S_{i j}^{d} S_{i j}^{d}\right)^{5 / 4}},
$$

where $C_{w}$ is the WALE model constant equal to $0.5, \Delta$ is the characteristic subgrid length scale, i.e., the mesh size, $\bar{S}_{i j}$ is the filtered strain rate tensor, and $S_{i j}^{d}$ is the symmetric part of the square of the filtered velocity gradient tensor $\bar{g}_{i j}=\partial \bar{u}_{i} / \partial x_{j}$. The term $S_{i j}^{d}$ accounts for both strain and rotation and can be rewritten in terms of the filtered strain rate and rotation tensors as such

$$
S_{i j}^{d} S_{i j}^{d}=\frac{1}{6}\left[\left(\bar{S}_{i j} \bar{S}_{i j}\right)^{2}+\left(\bar{\Omega}_{i j} \bar{\Omega}_{i j}\right)^{2}\right]+\frac{2}{3} \bar{S}_{i j} \bar{S}_{i j} \bar{\Omega}_{i j} \bar{\Omega}_{i j}+2 \bar{S}_{i k} \bar{S}_{k j} \bar{\Omega}_{j l} \bar{\Omega}_{l i} .
$$

The LES WALE model has been shown, by comparison to DNS of turbulent pipe flow, to reproduce suc- 
cessfully the correct near-wall scaling without additional damping functions and to model accurately the laminar-to-turbulent transition - both features that are important for the model setup investigated in this chapter.

\subsubsection{LES-Coupled heat transfer model}

Simulation of convective heat transfer due to turbulent fluid advection is accomplished by coupling the LES momentum equations to a thermal energy transport model. The governing thermal energy equation, derived from the First Law of Thermodynamics, is given by

$$
\frac{\partial e}{\partial t}+\nabla \cdot(\mathbf{u} e)=\frac{1}{\rho} \nabla \cdot(k \nabla T)-\frac{p}{\rho}(\nabla \cdot \mathbf{u})+\Phi_{\nu}
$$

where $e$ is the internal energy, $k$ is the fluid thermal diffusivity, $T$ is temperature, and $\Phi_{\nu}$ is viscous dissipation, i.e., internal heating through fluid viscosity [1]. Variable density effects are assumed to be negligible, and external sources of thermal energy (except for the fixed temperature hemisphere surface) are omitted. After grid-level filtering and applying the Boussinesq approximation, the thermal governing equation, where internal energy is represented by temperature, becomes

$$
\frac{\partial \bar{T}}{\partial t}+\frac{\partial\left(\bar{u}_{j} \bar{T}\right)}{\partial x_{j}}=\frac{\partial}{\partial x_{j}}\left(\mu \frac{\partial \bar{T}}{\partial x_{j}}-\overline{u_{j}^{\prime} T^{\prime}}\right) .
$$

Closure of the heat flux term $\overline{u_{j}^{\prime} T^{\prime}}$ is achieved by relating the mass eddy diffusivity $\epsilon_{M}$ (as specified by the SGS eddy viscosity model described above) to the heat eddy diffusivity, given by $\epsilon_{H}=\overline{u_{j}^{\prime} T^{\prime}} /\left(\partial \bar{T} / \partial x_{j}\right)$. The ratio of $\epsilon_{M}$ to $\epsilon_{H}$ defines the turbulent Prandtl number $P r_{t}$, which for all simulations is set equal to 0.9, a typical value for water. Thus, once $P r_{t}$ is specified, all the terms needed to calculate $\overline{u_{j}^{\prime} T^{\prime}}$ are known.

Heat transfer at the hemisphere wall is modeled using an automatic wall function, which employs a thermal law-of-the-wall description, developed by Kader (1981) [155], that blends the viscous sublayer and logarithmic layer profiles into a single non-dimensional temperature distribution $T^{+}$. The non-dimensional temperature is given by

$$
T^{+}=\operatorname{Pr} y^{*} e^{-\Gamma}+\left[2.12 \ln \left(y^{*}\right)+\beta\right] e^{(-1 / \Gamma)},
$$

where:

$$
\beta=\left(3.85 \operatorname{Pr}^{1 / 3}-1.3\right)^{2}+2.12 \ln (\operatorname{Pr}),
$$




$$
\Gamma=\frac{0.01\left(\operatorname{Pr} y^{*}\right)^{4}}{1+5 \operatorname{Pr}^{3} y^{*}}
$$

In the expressions for $\beta$ and $\Gamma, \operatorname{Pr}$ is the fluid Prandtl number given by $\operatorname{Pr}=\left(\mu c_{p}\right) / \lambda$, where $\mu$ is dynamic viscosity, $c_{p}$ is fluid heat capacity, and $\lambda$ is fluid thermal conductivity. $\operatorname{Pr}$ is distinct from the previously described turbulent Prandtl number $\operatorname{Pr}_{t}$, which is required for closure of the heat transfer turbulent flux equation. In equation 5.17, $y^{*}$ is a dimensionless near-wall length scale given by $y^{*}=\frac{u^{*} \Delta n}{\nu}$, where $\nu$ is kinematic viscosity, $\Delta n$ is the distance between the first and second grid points from the wall, and $u^{*}$ is log-layer velocity scale given by $u^{*}=C_{\mu}^{1 / 4} k^{1 / 2}$, where $C_{\mu}$ is model constant equal to 0.9 and $k$ is the turbulent kinetic energy. Once the non-dimensional temperature is determined, $T^{+}$can be used with the surface temperature $T_{S}$ and the near-wall fluid temperature $T_{f}$ to calculate the wall heat flux $q_{w}$, according to

$$
q_{w}=\frac{\rho c_{p} u^{*}}{T^{+}}\left(T_{S}-T_{f}\right)
$$

\subsubsection{Discretization and computational methods}

The flow features of greatest interest in this study are the hemisphere boundary layer and near-field wake structures and dynamics. In order to attain the highest fidelity for these features while maintaining a reasonable limit on computational expense, the fluid domain discretization was segmented into three regions of increasing mesh density. For unidirectional flows, a "high-resolution" box was positioned one-half hemisphere diameter in front of the model and extending 1.5 diameters downstream. The vertical range of the box extended one-half diameter above the model top. For oscillatory flows, the "high-resolution" box was centered over the hemisphere models, extending one model diameter in front of and behind the model. Additionally, in order to capture the highly sheared boundary layer, a "very high-resolution" sphere was positioned concentrically over each model, extending $1 \mathrm{~cm}$ normal to the model face. Figure 5.1 (right) shows the $w / k=0.5$ model domain, as an example, with the high-resolution regions visible. As an exception, the simple geometry of the smooth hemisphere allowed for the application of a surface inflation layer (25 layers extending $1 \mathrm{~cm}$ normal), rather than the concentric sphere, which reduced the number of required mesh elements while still maintaining high resolution of the shear layer.

For each model type, the main fluid domain was automatically discretized with tetrahedral elements using default sizing parameters, ranging from a minimum face size of $1.5 \mathrm{~cm}$ to a maximum of $3.2 \mathrm{~cm}$. The element size of the "high-resolution" box was assigned a maximum value $0.5 \mathrm{~cm}$, and the "super highresolution" concentric sphere was assigned a maximum element size of $0.1 \mathrm{~cm}$. As an example, figure 5.3 


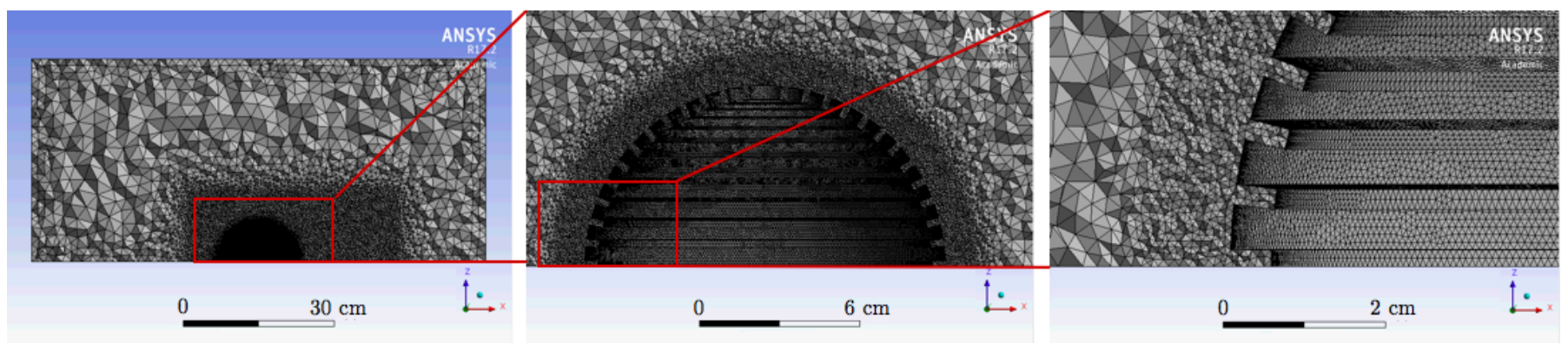

Figure 5.3: View of mid-plane slice at three levels of zoom illustrating the increasing mesh resolution for the flow areas of interest. Left. View of entire fluid domain mesh for unidirectional $w / k=0.5$ case. Middle. Zoomed view of "high-resolution" mesh region surrounding hemisphere model with maximum element size of $0.5 \mathrm{~cm}$. Right. Further zoom of "super high-resolution" concentric sphere mesh with maximum element size of $0.1 \mathrm{~cm}$.

shows a cross-sectional view of the resulting mesh for the $w / k=0.5$ hemisphere at three levels of zoom, corresponding to the three regions of increasing discretization. Mesh quality was determined based on average element skewness and orthogonal quality, with limiting criteria set to $<0.5$ and $>0.7$, respectively. Table 5.2 lists the total number of mesh elements generated for each model geometry.

The numerical procedures implemented to solve the governing equations were selected from standard p.d.e. solving strategies based on their appropriateness for LES turbulence flows. The advection terms of the governing equations were treated with a bounded central differencing scheme (CDS) because it has been shown to be less dissipative than alternative methods and provide good solutions within ANSYS CFX 156. Transient terms were treated with an implicit second-order backward Euler method, which avoids the overdamping of first-order methods. To improve solution accuracy, the simulation time step $\Delta t$ was adjusted for each run based on the peak flow velocity $u_{\max }$ and the smallest grid size $\Delta x_{\min }$ to maintain a CourantFriedrichs-Lewy (CFL) condition of approximately 1. Because the time integration scheme employed is an implicit technique, the CFL condition is not a criterion for numerical convergence, as would be the case for an explicit technique, but it does affect solution accuracy. The maximum CFL number $C_{\max }$ is given by

$$
C_{\max }=\frac{u_{\max } \Delta t}{\Delta x_{\min }}
$$

The time step required for $C_{\max } \sim 1$ for each run is given in Table 5.2 . Each simulation was run for a total of $60 \mathrm{~s}$. To allow for flow development and the convergence of turbulence statistics, flow and heat parameters of interest were averaged over the final $30 \mathrm{~s}$ of the simulation. Arranging the simulation timing in this manner allowed for six full wave cycles $(T=5 \mathrm{~s})$ to occur during flow setup for the oscillatory runs, and a further six full wave cycles to occur during parameter averaging. Because of the grid-based filtering procedure inherent to LES, the accuracy of a simulation can always be improved by refining the grid size - until the Kolmogorov scale is reached - at which point a DNS solution is achieved. Therefore, a formal analysis of increasing grid 


\begin{tabular}{ccccccc}
\hline Run & Geometry & Flow Regime & $u_{r m s}\left(\mathrm{~cm} \mathrm{~s}^{-1}\right)$ & Mesh Elements & $\Delta t(\mathrm{~s})$ & $R e$ \\
\hline \hline 1 & Smooth & Unidirectional & 5 & $1,427,023$ & 0.04 & 6,750 \\
2 & Smooth & Unidirectional & 15 & $1,427,023$ & 0.013 & 20,250 \\
3 & Smooth & Oscillatory & 5 & $1,429,259$ & 0.02829 & 6,750 \\
4 & Smooth & Oscillatory & 15 & $1,429,259$ & 0.00943 & 20,250 \\
& & & & & \\
5 & $w / k=0.5$ & Unidirectional & 5 & $5,349,661$ & 0.02 & 6,750 \\
6 & $w / k=0.5$ & Unidirectional & 15 & $5,349,661$ & 0.0066 & 20,250 \\
7 & $w / k=0.5$ & Oscillatory & 5 & $5,269,693$ & 0.02829 & 6,750 \\
8 & $w / k=0.5$ & Oscillatory & 15 & $5,269,693$ & 0.00943 & 20,250 \\
& & & & & & \\
9 & $w / k=2.0$ & Unidirectional & 5 & $5,643,656$ & 0.02 & 6,750 \\
10 & $w / k=2.0$ & Unidirectional & 15 & $5,643,656$ & 0.0066 & 20,250 \\
11 & $w / k=2.0$ & Oscillatory & 5 & $5,643,819$ & 0.02829 & 6,750 \\
12 & $w / k=2.0$ & Oscillatory & 15 & $5,643,819$ & 0.00943 & 20,250 \\
& & & & & & \\
13 & Coral & Unidirectional & 5 & $9,208,147$ & 0.02 & 6,750 \\
14 & Coral & Unidirectional & 15 & $9,208,147$ & 0.0066 & 20,250 \\
15 & Coral & Oscillatory & 5 & $9,261,241$ & 0.02829 & 6,750 \\
16 & Coral & Oscillatory & 15 & $9,261,241$ & 0.00943 & 20,250 \\
\hline
\end{tabular}

Table 5.2: Description of free-stream velocities, corresponding Reynolds numbers, and resulting flat-plate boundary layer thicknesses for experimental flows over coral models.

sensitivity was not undertaken; rather, the numerical model was validated against the experimental PIV results of Chapter 4 .

\subsection{Model Validation}

To establish the validity of the numerical model, mean and turbulent flow quantities were compared to calculated values from the empirical water tunnel data. Profiles of mean horizontal velocity $\bar{u}$ were selected for comparison at four critical streamwise locations: 1 ) at the model leading edge, $x=-4.5 \mathrm{~cm}$; 2 ) at the model top, $x=0 \mathrm{~cm}$; 3 ) in the very near downstream wake just beyond the model trailing edge, $x=8.75 \mathrm{~cm}$; and 4) in the recirculating wake one-half model diameter downstream, $x=13.5 \mathrm{~cm}$. Figure 5.4 shows the locations of the investigated profiles as dashed lines within a schematic of the experimental PIV domain. For clarity, mean velocity comparisons are shown only for the smooth hemisphere model and the $w / k=0.5$ roughness model, which was chosen as a representative of all three rough model types.

Figure 5.5 shows the comparison for the low-flow test case, i.e., $R e=6,750$, with the LES model output as a solid line and the PIV experimental data as circle markers. Excellent agreement is seen for both surface geometry types across all four profile locations, even in regions of high shear such as at the model surface boundary layer and in the downstream wake. Figure 5.6 shows the comparison for the high-flow test case, i.e., $R e=20,250$. Several locations exhibit significant deviation between the profiles, most notably at 


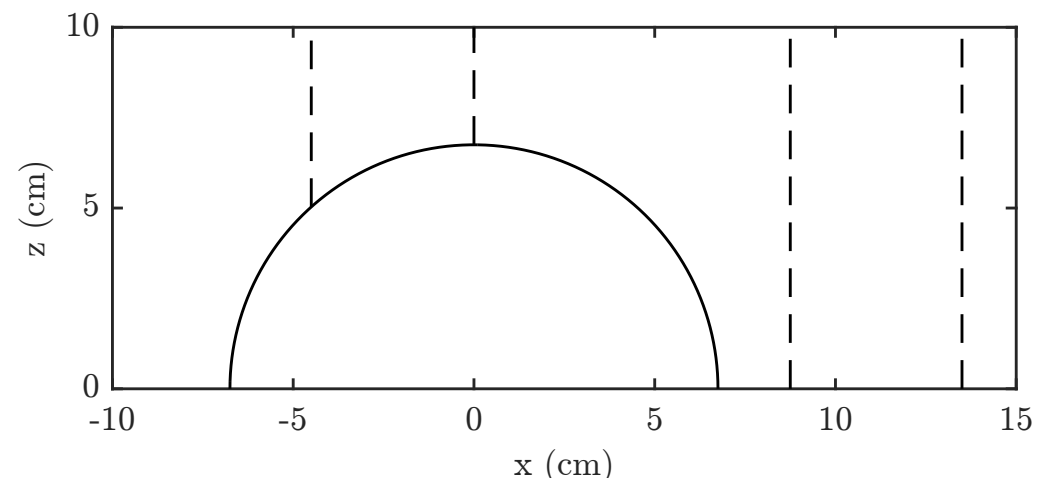

Figure 5.4: Streamwise locations of vertical profiles (indicated by dashed vertical lines) used for validation of LES model.
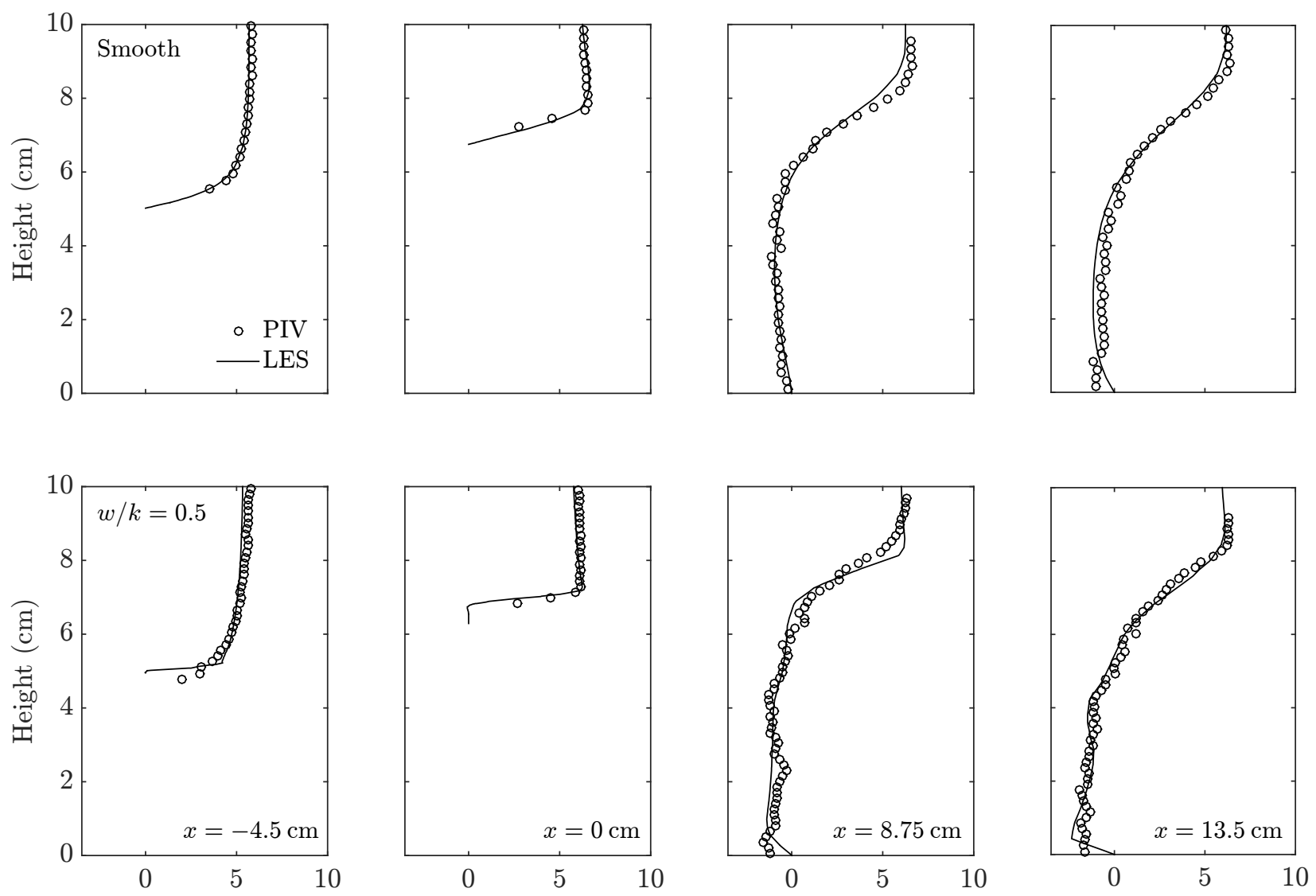

$\bar{u}\left(\mathrm{~cm} \mathrm{~s}^{-1}\right)$

Figure 5.5: Profiles of mean horizontal velocity $\bar{u}$. Top. Low-flow condition: $u_{\infty}=5 \mathrm{~cm} \mathrm{~s}^{-1}, R e=6,750$. 
the rough model top and in all the downstream plots at elevations below the shear layer. The discrepancy is most likely attributable to the difficulty of the experimental PIV setup in obtaining quality data within regions of highly turbulent, three-dimensional flow, such as the bluff body near-wake region.

In the highly three-dimensional flow of the near-wake region, PIV imaging particles move rapidly in and out of the two-dimensional laser plane, creating an inherent limitation in the image-pair pattern matching required to estimate velocities. A large body of historical research on flow past cylinders and spheres, e.g., 113, 122, 157, 158, demonstrates that the near-wake region should possess a recirculating flow with mean negative horizontal velocity - a result seen in the LES results, but not present in the PIV data ${ }^{2}$ Further, due to the limited acquisition rate (24 frames-per-second) of the DSLR camera used in the PIV

\footnotetext{
${ }^{2}$ Regarding the validity of the conclusions drawn in Chapter 4 , those results relied primarily on the accurate boundary layer and shear layer data, rather than the potentially biased recirculating, near-wake data.
}
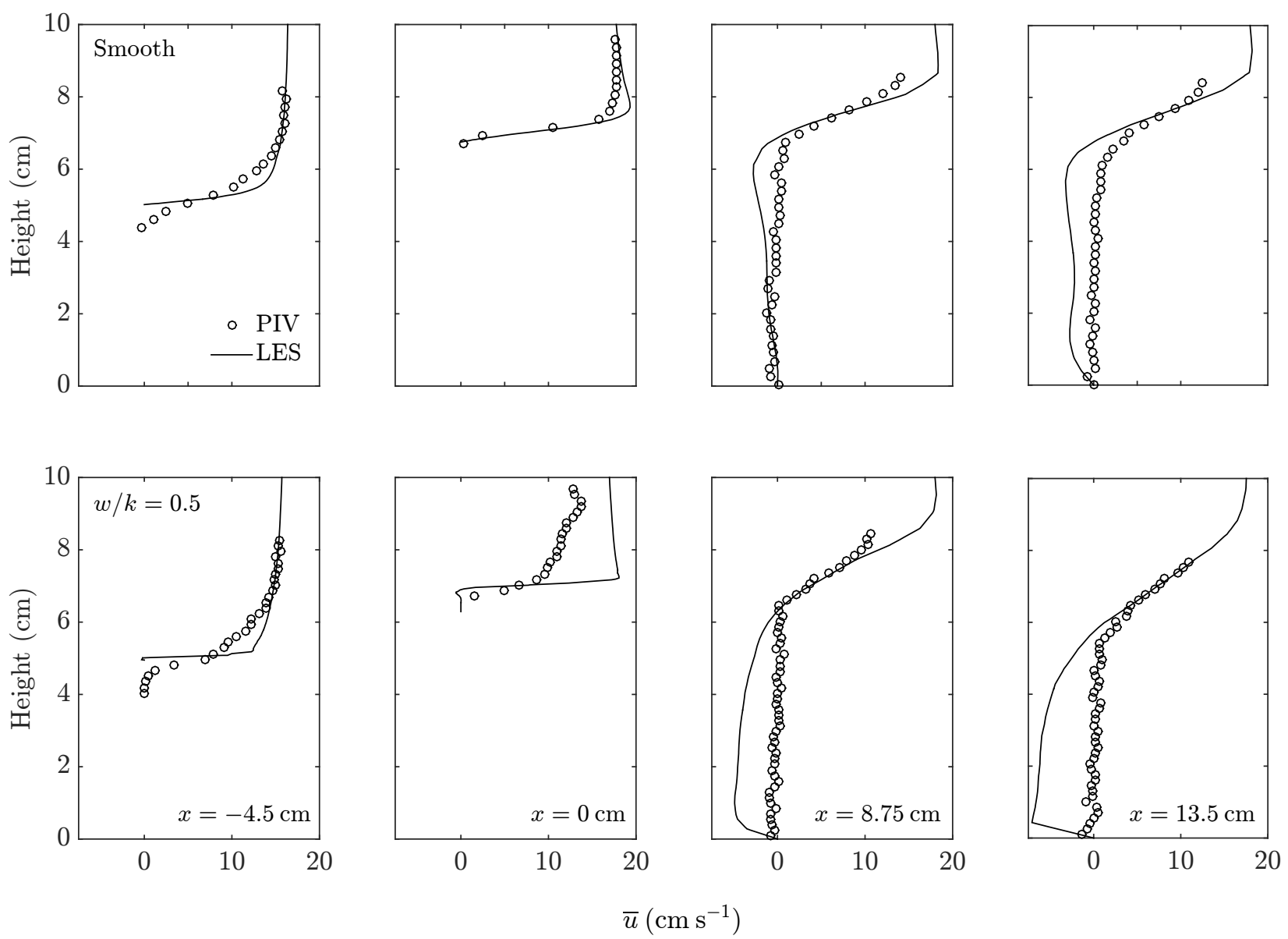

Figure 5.6: Profiles of mean horizontal velocity $\bar{u}$. Top. High-flow condition: $u_{\infty}=15 \mathrm{~cm} \mathrm{~s}^{-1}, R e=20,250$. 

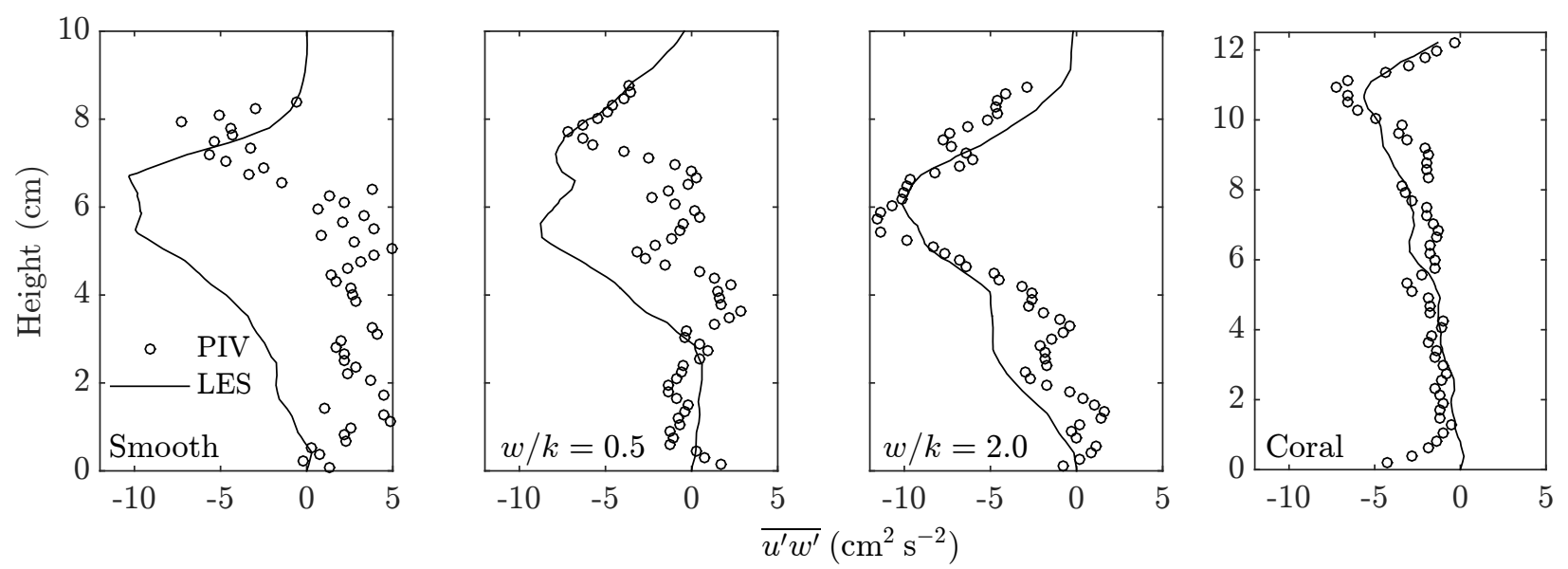

Figure 5.7: Reynolds shear stress.

setup - as opposed to a more standard, more expensive commercial system with frame rates up to 4,000,000 fps - the high velocity test case approached the limits of the system's ability to measure velocity accurately. The system has previously been shown to scale with free-stream velocity, according to $\pm 6 \%$ of $u_{\infty}$ [106], and thus, the fast-flow regime exhibits a greater deviation from the numerical model compared to the slow-flow regime primarily because of experimental error.

Additionally, comparisons of turbulent Reynolds shear stress profiles were used to validate the numerical model outputs. Figure 5.7 plots numerical model and experimental Reynolds shear stresses for all four hemisphere geometries at $R e=20,250$ at the far downstream profile location, $x=13.5 \mathrm{~cm}$. As described above, the downstream high-flow results are valid above and within the shear layer, but are less reliable in the recirculating zone below approximately $z=6 \mathrm{~cm}$. Peak Reynolds stresses compare well in both location and magnitude for all three rough hemispheres, while the smooth experimental results are approximately $2.5 \mathrm{x}$ greater than the model output. This is likely due to the prescribed inlet flow conditions of the numerical model, which introduce purely laminar flow at the boundary condition, while the inlet water tunnel flow contains residual background turbulence. Interaction with the model surface amplifies these fluctuations creating the larger shear stress signal in the PIV data. Results are shown only for the high-flow regime because the shear stress levels generated by the low-flow regime were so small as to be masked by the experimental error noise. 


\section{$5.4 \quad$ Numerical Results}

Numerical model results describing flow characteristics will be presented first, followed by results of temperature fields and heat transfer. Each major results section will be subdivided into two parts, which describe: 1) the far-field unidirectional and oscillatory results, and 2) the near-surface unidirectional and oscillatory results.

\subsubsection{Results: Flow Characteristics}

\section{Far-field flow results}

Mean horizontal velocities $\bar{u}$ for all four surface roughnesses at $R e=6,750$ and $R e=20,250$ are shown in figure 5.8 for the unidirectional regime and in figure 5.9 for the oscillatory regime. The cutplane shown is taken from the cross-stream midline, i.e., $y_{\text {plane }}=1 / 2 y_{\max }$. For the unidirectional case, flow direction in all plots is from left to right, and for the oscillatory case, flow is driven sinusoidally from the left inlet while allowing flow re-entry at the opening at right. The velocity legends for the low and high $R e$ regimes are given in dimensional terms, but the ranges between maximum and minimum velocities are scaled identically so that the colorbar scaling between the two Reynolds numbers can be considered non-dimensional.

Under unidirectional flow, the velocity fields, as expected, all exhibit separation points near the model top and reattachment downstream to create an area of recirculation behind the model bluff body. The flow fields among the idealized models are topologically similar within each $R e$ regime, but show a shortening of the reattachment length at high Reynolds numbers. The wake structure of the coral model deviates from the idealized models owing to the large-scale morphological asymmetries. Under oscillatory flow, the broad k-type surface roughness produces a markedly different flow structure compared to the other models, with greater perturbation of the organized wake topology and significant non-zero velocities advected vertically well above the model top. The envelope of high-velocity boundary layer flow extends outward nearly $4 \mathrm{x}$ farther from the k-type model surface compared to the other models, indicating the role of roughness-induced turbulence in sustaining residual flow.

To quantify the size and strength of the wake recirculation zones, horizontal profiles of streamwise velocity were extracted from each field plot at a vertical height of $1.5 \mathrm{~cm}$ above the bottom wall- the vertical location, as demonstrated in previous experimental work on hemispheres [158, closest to the region of greatest recirculation while still avoiding wall drag effects. Figure 5.10 plots these velocities - normalized by free-stream velocity - for each surface roughness at low and high Reynolds number conditions under steady and unsteady forcing. The recirculation length is determined by the location on the x-axis at which velocity 

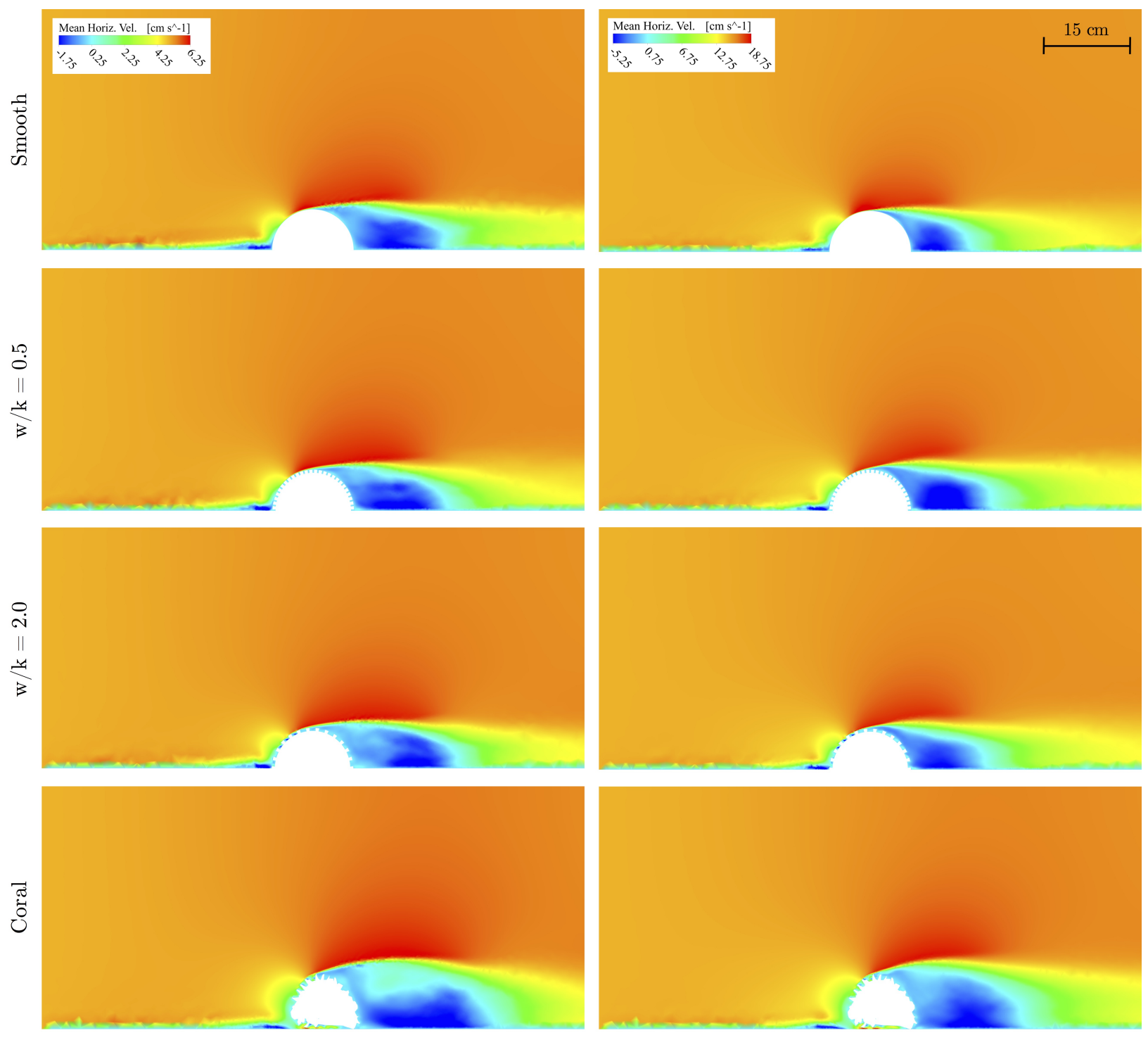

$R e=6,750$

$R e=20,250$

Figure 5.8: Time-averaged streamwise velocity fields for low (left) and high (right) Re unidirectional flow test cases. Maximum and minimum colorbar values are scaled proportionally across each Reynolds number regime so that color field plots are non-dimensional.

transitions from negative to positive, and the recirculation strength is measured by the peak value of negative flow. For both unidirectional cases, the smooth model exhibited the shortest recirculation length, due to the lack of flow disturbance caused by surface roughness features. At $R e=6,750$, the recirculation bubbles for the $w / k=2.0$ roughness and coral model are approximately $25 \%$ larger, though recirculation strength is approximately equal for all roughness types. At $R e=20,250$, the models with surface roughness exhibit an increase in reattachment length of approximately $50 \%$ compared to the smooth model, but interestingly, the k-type roughness recirculation strength is only about $70 \%$ of the peak recirculation of the d-type roughness- 

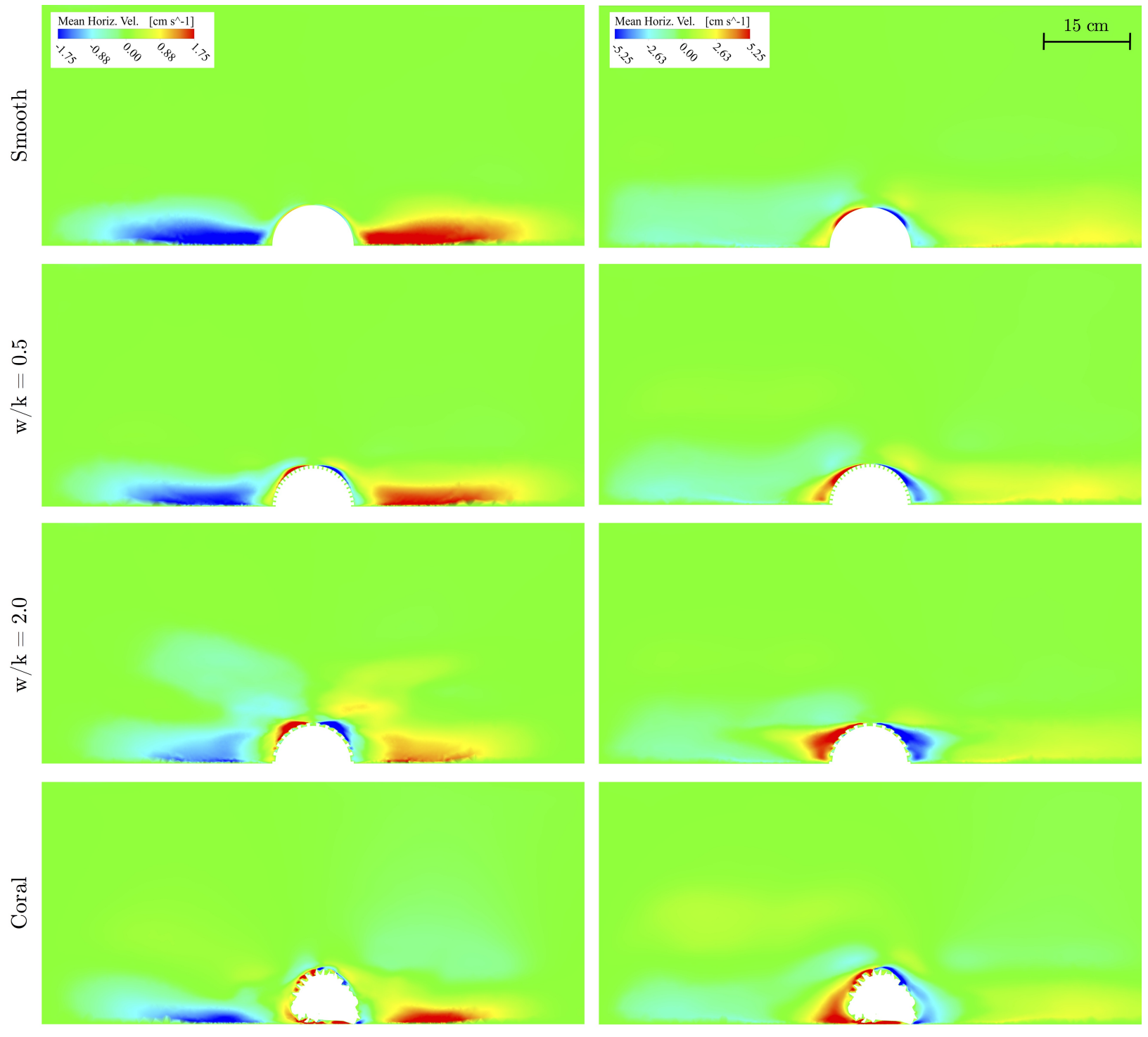

$R e=6,750$

$R e=20,250$

Figure 5.9: Time-averaged streamwise velocity $\bar{u}$ fields for low (left) and high (right) Re oscillatory flow test cases.

a dimensional deficit of nearly $2 \mathrm{~cm} \mathrm{~s}^{-1}$. This reduction in recirculation strength extends vertically across the entire height of the recirculation bubble as demonstrated in figure 5.11, which plots vertical profiles of streamwise velocity taken within the middle of the wake at $x / D=0.5$.

The bottom row in figure 5.10 shows the horizontal profiles of mean streamwise velocity for the oscillatory test cases. The key distinction from the unidirectional regime is that the unsteady nature of the oscillatory flow prevents a stable recirculation bubble from establishing itself. Rather, the surface roughness acts to smear out sharp gradients in mean velocity in the far-field wake, with the amount of dispersion increasing with larger $w / k$ roughness ratios. This is evident in the lower velocity magnitudes for the $w / k=2.0$ 

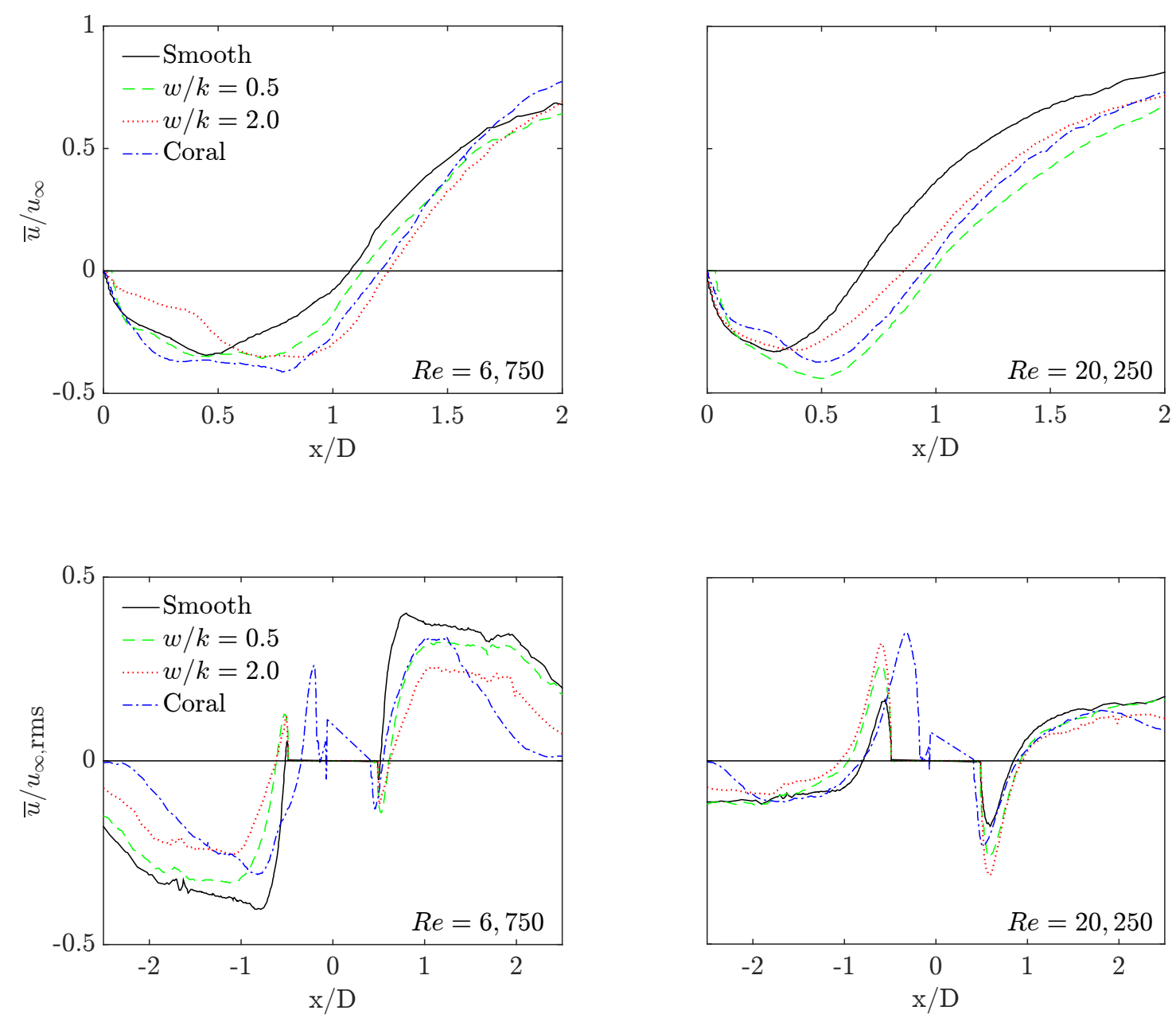

Figure 5.10: Horizontal profiles of mean streamwise velocity $\bar{u}$ (normalized by free-stream velocity $u_{\infty}$ ) at $z=1.5 \mathrm{~cm}$ above bottom wall. Top row: Unidirectional flow test case. Bottom row: Oscillatory flow test case.

model in the far-field regions 1-2 diameters away from the model surface for both low and high Reynolds number regimes.

Statistical Reynolds shear stresses $\overline{u^{\prime} w^{\prime}}$ are automatically calculated by the numerical model using running means of the instantaneous velocity correlation and running means of instantaneous velocities, according to $\overline{u_{i}^{\prime} w_{j}^{\prime}}=\overline{u_{i} u_{j}}-\overline{u_{i}} \overline{u_{j}}$. Figure 5.12 plots mean Reynolds shear stresses $\overline{u^{\prime} w^{\prime}}$ for the unidirectional test cases, and figure 5.13 shows $\overline{u^{\prime} w^{\prime}}$ for the oscillatory test cases. Again, so that non-dimensional comparisons between Reynolds number regimes can be made, maximum and minimum Reynolds shear stresses are scaled proportionally in the colorbars (since $\overline{u^{\prime} w^{\prime}}$ scales with the square of velocity, peak colorbar values are $9 \mathrm{x}$ larger in the high $R e$ case to reflect the $3 \mathrm{x}$ velocity difference). In the unidirectional plots, the distribution of $\overline{u^{\prime} w^{\prime}}$ is largely similar across the different roughness models, with the key distinction being a broader spread 

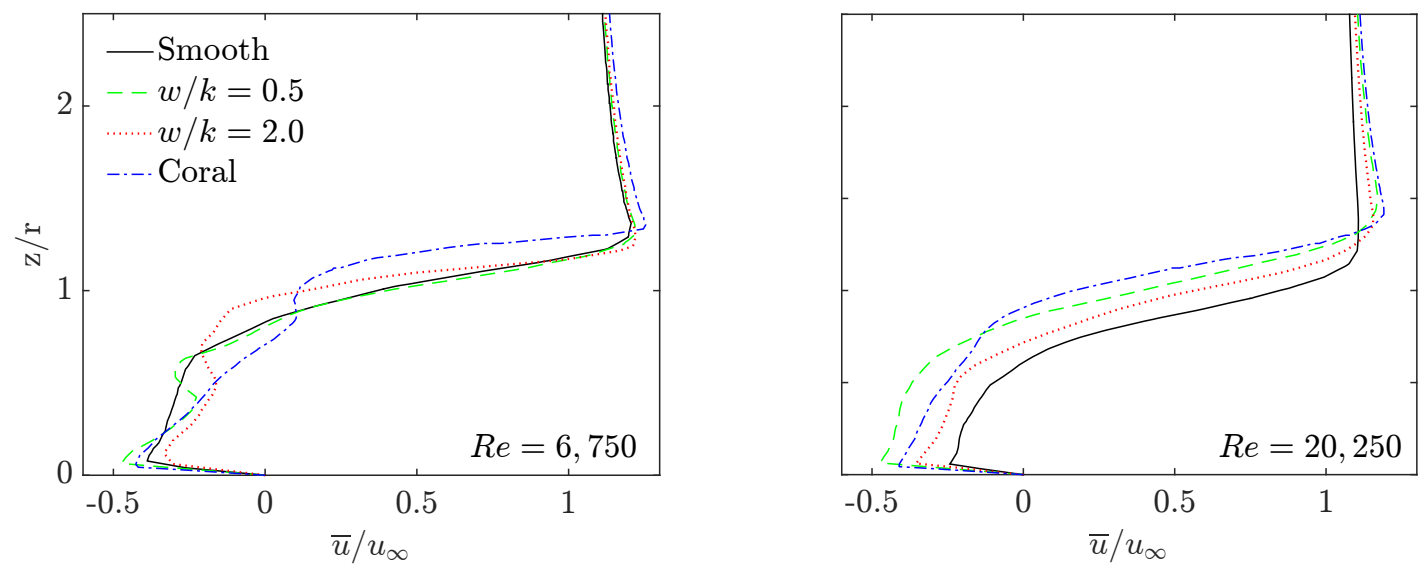

Figure 5.11: Vertical profiles of mean streamwise velocity $\bar{u}$ (normalized by free-stream velocity $u_{\infty}$ ) for $R e=6,750$ (left) and $R e=20,250$ (right) at one-half diameter downstream from trailing edge of model, i.e., $x / D=0.5$, under unidirectional flow.

of shear stresses both vertically and nearer to the model surface for the high Reynolds number flows. For the oscillatory plots, the k-type surface roughness clearly disturbs the stress distribution pattern occurring at the model top, causing a breakup of the well-defined lobes seen in the other models and greater advection of non-zero stresses to regions farther away from the peak shear producing location of the model top.

To compare $\overline{u^{\prime} w^{\prime}}$ values quantitatively, streamwise profiles were extracted along the horizontal line level with the top of each model, i.e., $z / r=1$, where the Kelvin-Helmholtz instabilities of the wake shear layer and vortices shed from roughness elements generate peak stress values as confirmed visually in the field plots. Figure 5.14 plots normalized Reynolds shear stresses for the unidirectional test cases (top row) from the model top to the domain end and for the oscillatory test cases (bottom row) across the entire model domain. For both low and high Re unidirectional flows, the k-type surface roughness produces approximately $20 \%$ larger peak shear stress values compared to the d-type surface, as well as a significantly larger "tail" of nonzero $\overline{u^{\prime} w^{\prime}}$ in the low $R e$ regime. Peak values shift closer to the model surface at the larger Reynolds number, moving from roughly 0.7 diameters downstream to only about 0.4 diameters downstream. By contrast, under oscillatory forcing, the peak Reynolds stress values associated with roughness type are reversed, with the smooth and d-type models exhibiting nearly $50 \%$ greater values of $\overline{u^{\prime} w^{\prime}}$. Interestingly, at high $R e$ oscillatory flow, the Reynolds stress profiles for the smooth and d-type models are nearly indistinguishable, suggesting that the roughness elements contribute very little to the production of shear stresses under these conditions.

The turbulence dissipation rate $\epsilon$ - the rate at which viscosity converts turbulent kinetic energy into heat - correlates to the degree of "smearing out" of scalar and thermal gradients, with larger $\epsilon$ more 

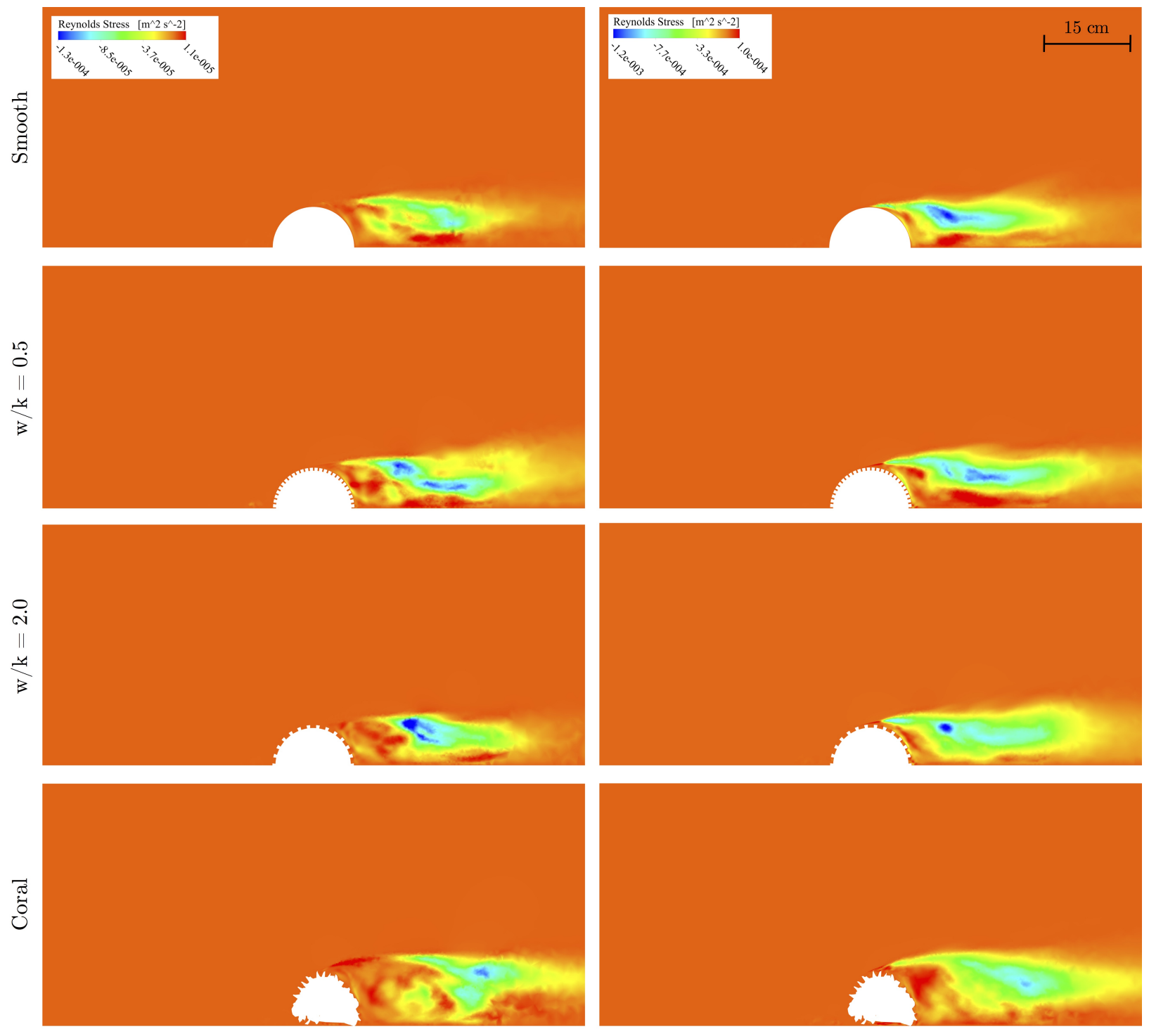

$$
R e=6,750
$$

$$
R e=20,250
$$

Figure 5.12: Field plots of statistical Reynolds shear stress $\overline{u^{\prime} w^{\prime}}$ for low (left) and high (right) Re unidirectional flow test cases. Maximum and minimum colorbar values are scaled proportionally across each Reynolds number regime so that color field plots are non-dimensional.

effectively reducing velocity gradients and homogenizing a scalar/thermal field. Turbulence dissipation is a field parameter that varies spatially, depending turbulence production and transport, and is defined by $\epsilon=2 \nu \overline{S_{i j} S_{i j}}$, where $S_{i j}$ is the strain rate tensor. Table 5.3 gives the rate of turbulence dissipation for each model type and flow condition at a point location one model diameter downstream and one model height above the wall. The dissipation rates show that the unidirectional flow advects turbulence to the downstream point more effectively than does the oscillatory flow, as expected from the flow dynamics, and that the higher $R e$ regimes produce more intense turbulent mixing. $\epsilon$ is also used to define the smallest turbulent length 

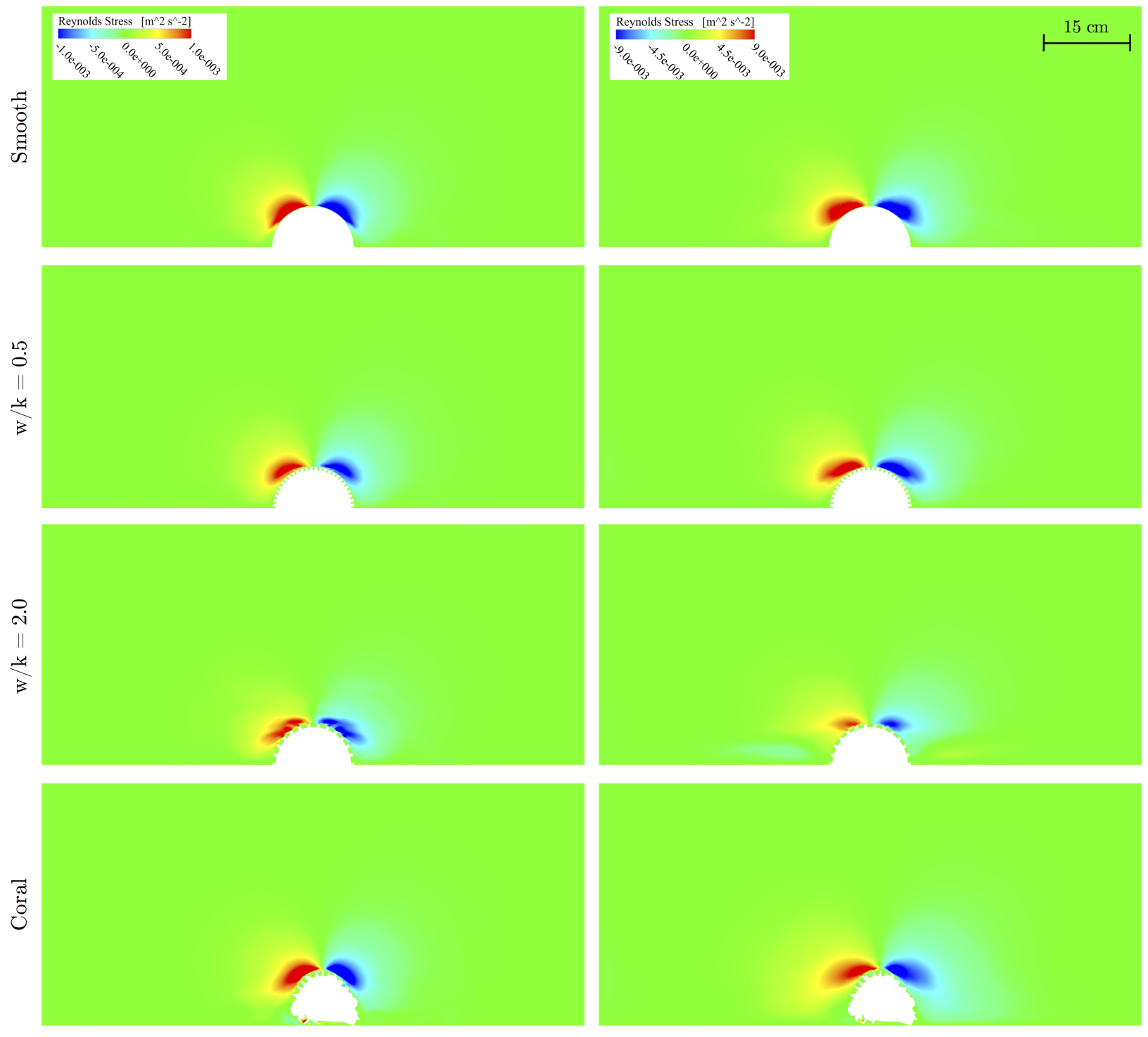

$R e=6,750$

$R e=20,250$

Figure 5.13: Field plots of statistical Reynolds shear stress $\overline{u^{\prime} w^{\prime}}$ for low (left) and high (right) Re oscillatory flow test cases.

scale, called the Kolmogorov microscale $\eta$, according to $\eta=\left(\nu^{3} / \epsilon\right)^{1 / 4}$. Since $\eta$ is inversely proportional to $\epsilon$, the larger dissipation rates create smaller scale eddies, as seen in table 5.3 , which provide the mechanism for more effectively removing velocity gradients.

\section{Near-surface flow results}

The far-field hydrodynamic results presented above play a critical role in scalar and thermal mixing and advection for the downstream environment. However, in order to understand and quantify the scalar and thermal flux occurring at the model surface, it is necessary to investigate near-field boundary layer 

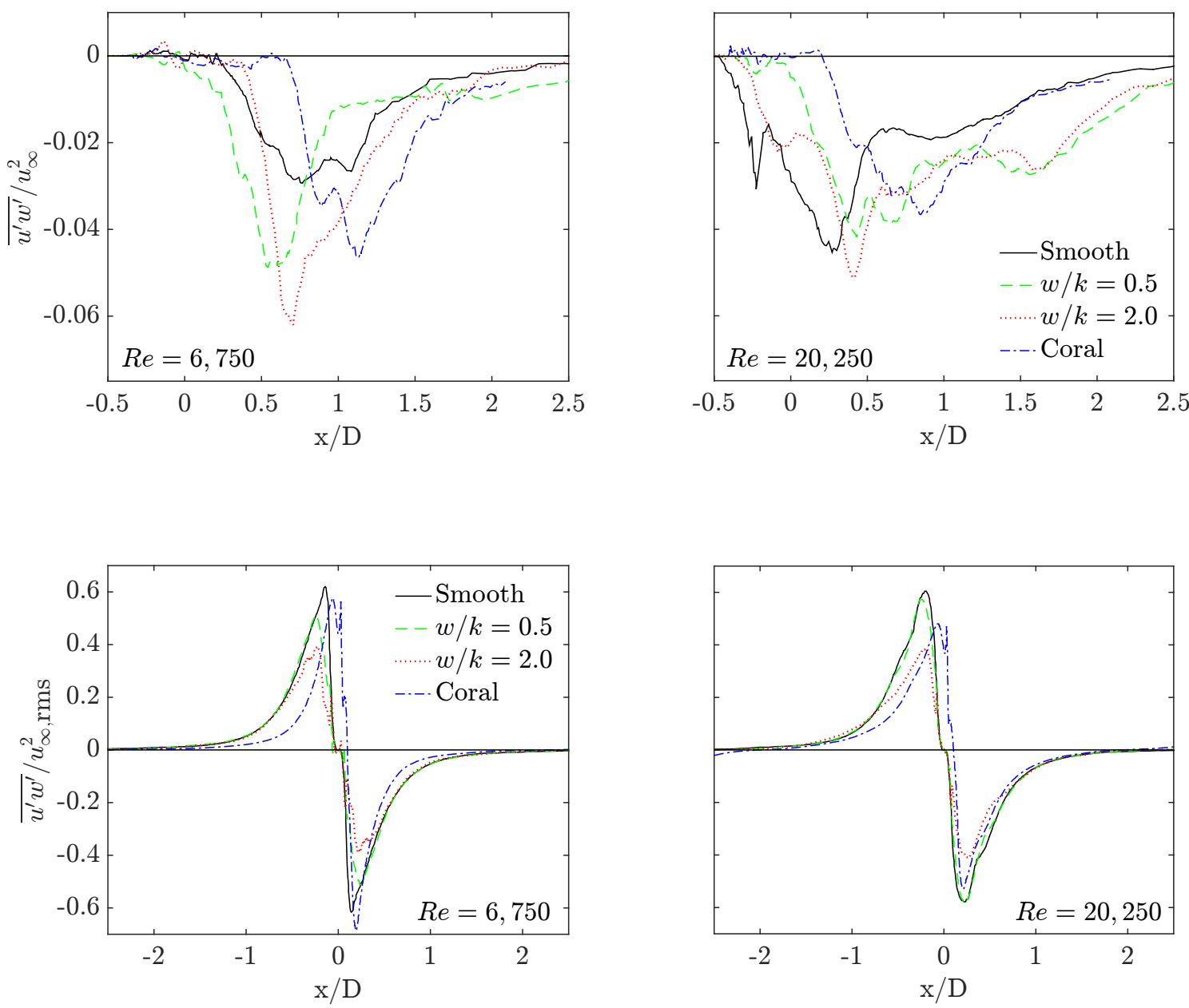

Figure 5.14: Horizontal profiles of mean Reynolds shear stress $\overline{u^{\prime} w^{\prime}}$ (normalized by the square of the free-stream velocity $u_{\infty}^{2}$ ) at an elevation equal to half the model height, i.e., $z / r=0.5$. Top row: Unidirectional flow test case. Bottom row: Oscillatory flow test case.

characteristics and flow fields both above and within surface roughness elements. Figure 5.15 shows close-up, near-surface plots of mean total velocity magnitude for each surface roughness, Reynolds regime, and flow test case. Three-component velocity magnitude, rather than streamwise-only velocity, is plotted because it provides a clearer visualization of the fluid motion occurring within the roughness cavities and because flow in any direction adjacent to the cavity walls - not just horizontal flow — plays a role in heat flux from the surface. Colorbar legends are again scaled proportionally for low and high $R e$ regimes to generate non-dimensional plot coloring.

For the unidirectional case $(t o p)$, each model shows a zero-velocity stagnation point at the leading edge, approximately $3 \mathrm{~cm}$ and $2.5 \mathrm{~cm}$ above the bottom wall for the low and high $R e$ regimes, respectively. 
Turbulence dissipation rate, $\epsilon \times 10^{-6}\left(\mathrm{~m}^{2} / \mathrm{s}^{3}\right)$

\begin{tabular}{lrcccc}
\hline Flow regime & $R e$ & Smooth & $w / k=0.5$ & $w / k=2.0$ & Coral \\
\hline \hline Unidirectional & 6,750 & 6.86 & 5.89 & 7.32 & 5.71 \\
Oscillatory & 6,750 & 0.11 & 0.03 & 0.08 & 0.02 \\
Unidirectional & 20,250 & 21.9 & 60.4 & 39.2 & 44.7 \\
Oscillatory & 20,250 & 3.37 & 2.54 & 6.48 & 0.46 \\
\hline \multicolumn{6}{c}{} \\
\hline \multicolumn{7}{c}{ Kolmogorov microscale, $\eta(\mathrm{mm})$} \\
\hline Flow regime & $R e$ & Smooth & $w / k=0.5$ & $w / k=2.0$ & Coral \\
Unidirectional & 6,750 & 0.53 & 0.55 & 0.52 & 0.56 \\
Unididirectional & 6,750 & 1.51 & 2.14 & 1.59 & 2.45 \\
Oscillatory & 20,250 & 0.40 & 0.31 & 0.34 & 0.33 \\
\hline
\end{tabular}

Table 5.3: Turbulence characteristics for each roughness type and flow condition at a point location one model diameter downstream, $x / D=1$, and one model height above the wall, $z / r=1$.

Close inspection of the leading edge shows almost no flow entering the narrow, d-type roughness cavities at low Reynolds number, while at high Reynolds number, moderate penetration into the d-type roughness cavities occurs in a small region (an arc length of approximately $45^{\circ}$ ) just above the stagnation point. For the broadly spaced k-type roughness, intense vortical flow fully penetrates the leading edge roughness cavities for both low and high Reynolds number regimes, and lower magnitude vortical flow begins to develop within trailing edge cavities, driven by the strength of the recirculating flow at high Re. Similarly, the coral model shows flow fully penetrating the leading edge cavities for both Reynolds regimes, though with less organized structure owing to the less regular roughness structure and large scale morphological asymmetries.

Under oscillatory forcing, the intra-cavity flow dynamics are significantly altered. The unsteady flow conditions drive non-zero mean velocities into nearly every roughness cavity on both the leading and trailing edges for all roughness models. The main distinction is the depth of penetration. Whereas the k-type and coral models show flow clearly reaching the cavity bottoms for both low and high $R e$ flows, the d-type roughness exhibits a qualitative difference in the degree of flow penetration, with the higher velocity flow, as expected, pushing non-zero velocity further into the roughness cavities and exposing additional surface area to convective flow.

To quantify the penetration depth $h_{p}$ and strength of intra-cavity flow, surface normal profiles of the horizontal component of velocity were taken at the middle of the models' leading edges (approximately $45^{\circ}$ from the base of the model front), beginning at the bottom of the roughness cavity and extending normal to the surface for $2 \mathrm{~cm}$. The streamwise velocity was selected because it more clearly illustrates the strength of vortical flow within the cavities. Figure 5.16 plots surface normal profiles for each $R e$ regime and flow condition, normalized by free-stream velocity. The dashed zero line of the vertical z-axis represents the top of the roughness cavity for the d-type, k-type, and coral models and the model surface for the smooth 


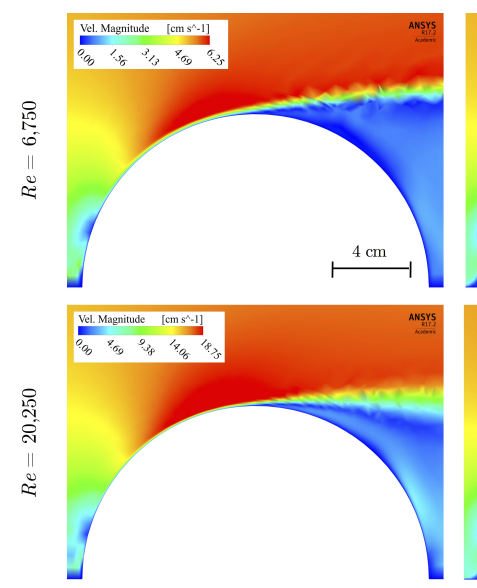

Smooth
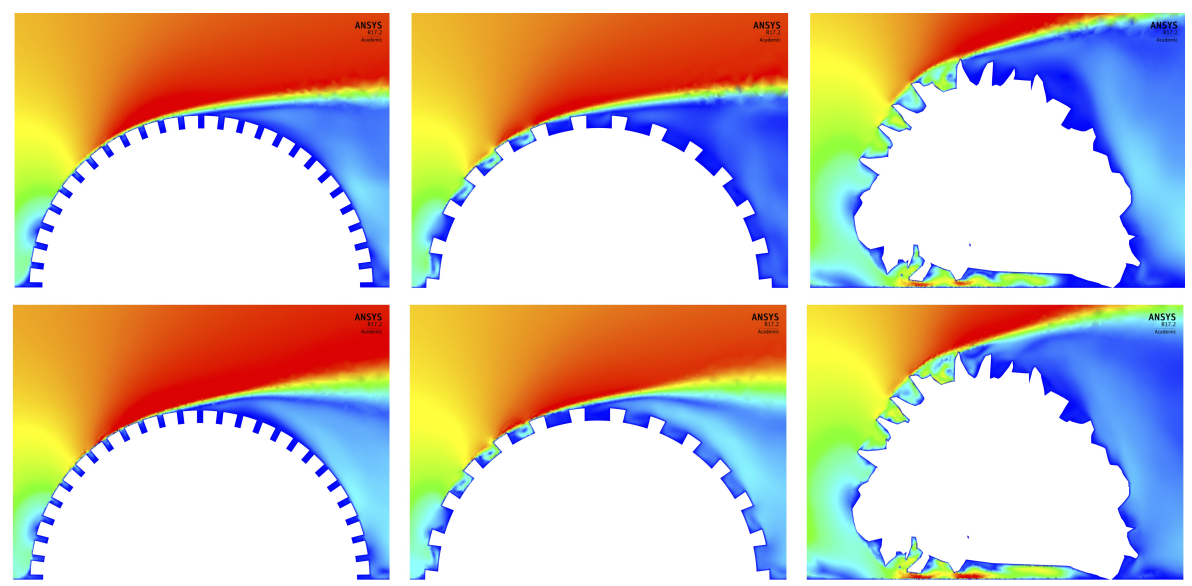

$\mathrm{w} / \mathrm{k}=0.5$

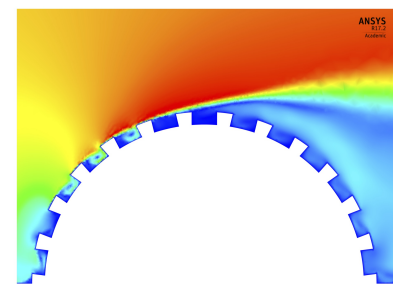

$\mathrm{w} / \mathrm{k}=2.0$

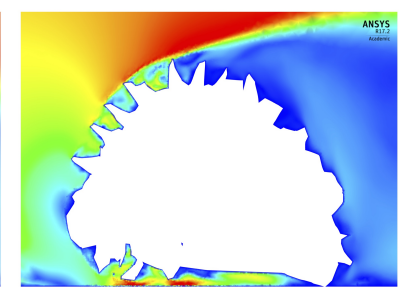

Coral

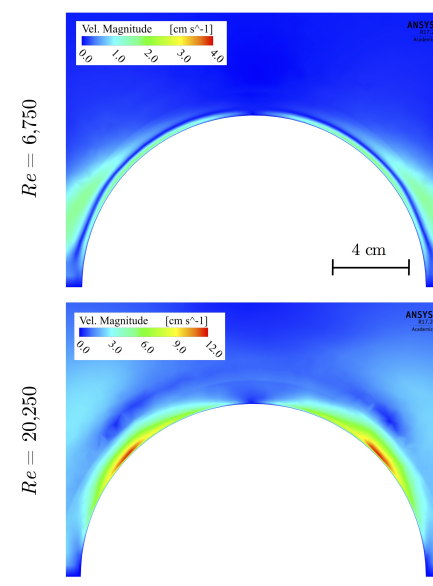

Smooth
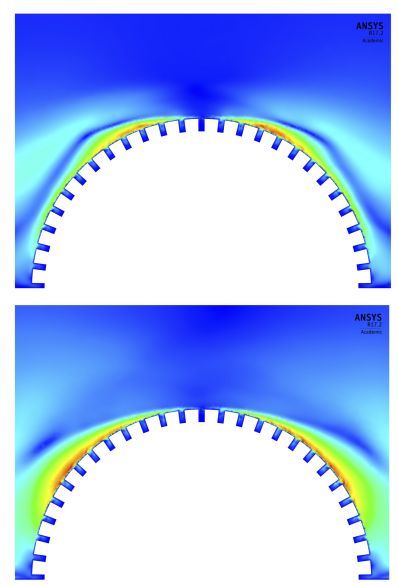

$\mathrm{w} / \mathrm{k}=0.5$
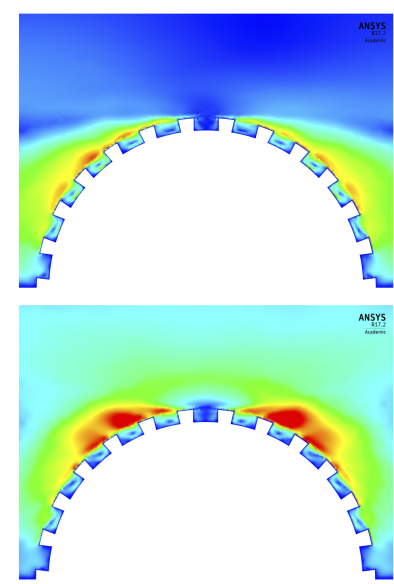

$\mathrm{w} / \mathrm{k}=2.0$
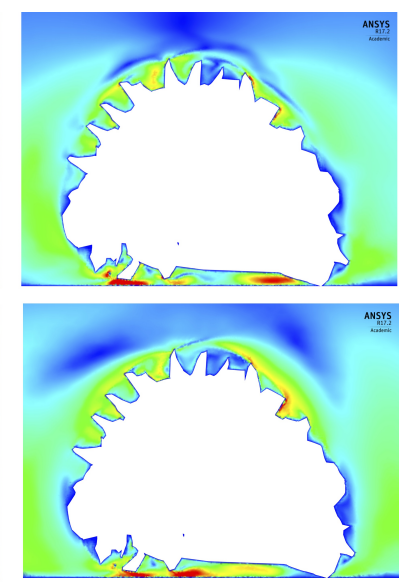

Coral

Figure 5.15: Near-surface close-up field plots of mean velocity magnitude $\overline{\mathbf{u}}$ for unidirectional test cases (top) and oscillatory test cases (bottom) for low and high Re regimes. Maximum and minimum colorbar values are scaled proportionally across each Reynolds number regime so that color field plots are non-dimensional.

hemisphere. As seen in the two-dimensional plots, the k-type surface roughness generates very strong negative flows - nearly $50 \%$ of $u_{\infty}$, except for the low oscillatory condition - directly adjacent to the cavity floor. The d-type roughness, by contrast, exhibits very small negative flows - only about $5 \%$ of $u_{\infty}$. The penetration depth for the d-type roughness, as measured by the location at which negative velocity strength drops below $0.1 \mathrm{~cm} \mathrm{~s}^{-1}$, is approximately $0.3-0.35 \mathrm{~cm}$ for the low $R e$ regimes, and approximately $0.4 \mathrm{~cm}$ for the high $R e$ regime. In other words, for the d-type model, $h_{p}$ extends roughly $60-70 \%$ of the cavity depth for low $R e$ flows and $\sim 80 \%$ of the cavity depth for high Re flows. Whereas, the k-type model has a $100 \%$ penetration depth for all flow conditions.

Figure 5.17 plots the mean wall surface shear stress for unidirectional (top) and oscillatory (bottom) 

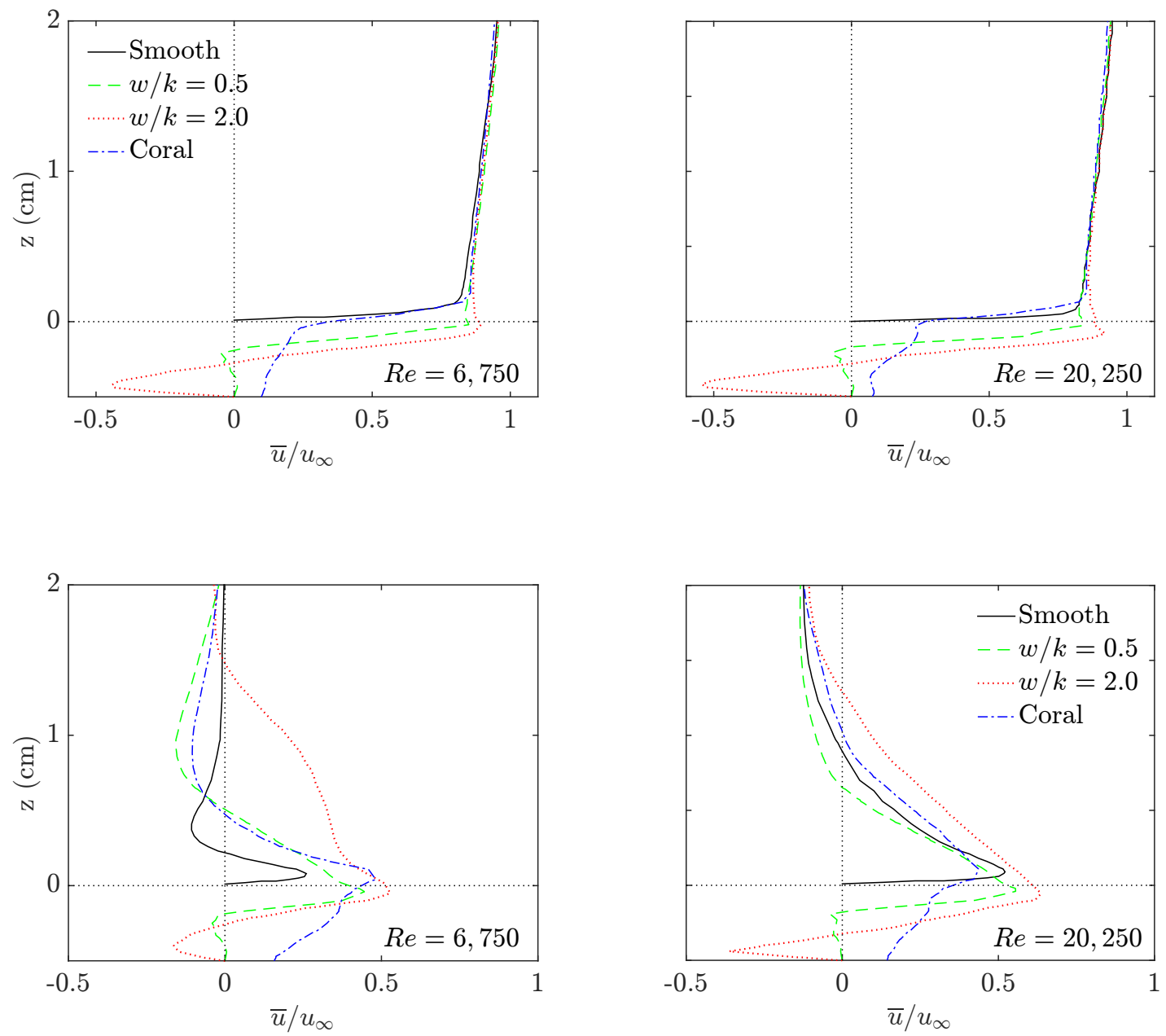

Figure 5.16: Surface normal profiles of mean streamwise velocity $\bar{u}$ (normalized by free-stream velocity $u_{\infty}$ ) for unidirectional flow (top) and oscillatory flow (bottom). Profiles are taken at the model leading edge at a location $45^{\circ}$ from the front base and extend outward from the bottom of the roughness cavity.

test cases at both low and high Reynolds number. As expected, under unidirectional forcing, the model leading edges' experience the greatest shear, increasing from a minimum at the stagnation point to a maximum in the area of greatest flow compression, and again decreasing to the point of flow separation. The trailing edges exhibit negligible surface shear, except for a small region near the model bottom where high $R e$ recirculating flow interacts with the model surface. For the rough models, peak shear values occur at the edges or tips of individual roughness elements, which become most evident on the coral model with its highly convoluted roughness patterning. Under oscillatory flows, peak stresses are lower than for comparable unidirectional flow speeds, but the surface area exposed to non-negligible shear stresses is approximately double that of the unidirectional case. Thus, a trade-off between wall shear strength and areal wall shear exposure is established 


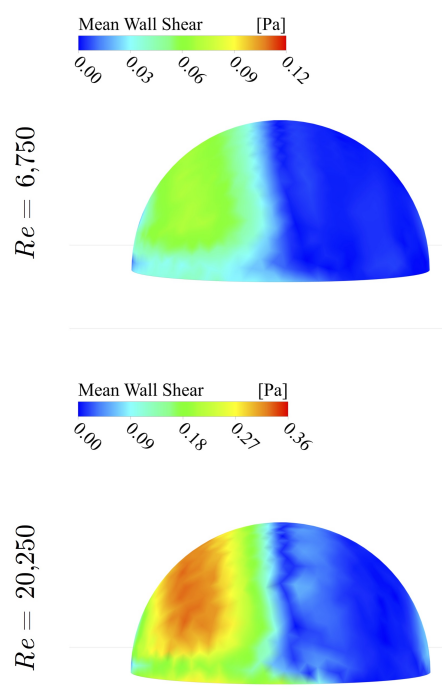

Smooth

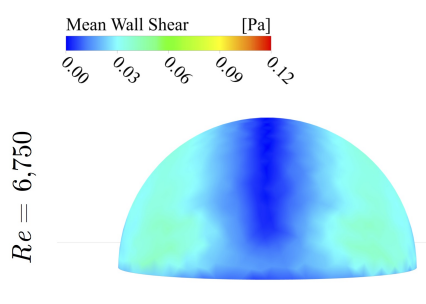

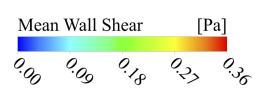

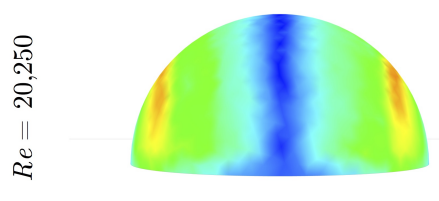

Smooth
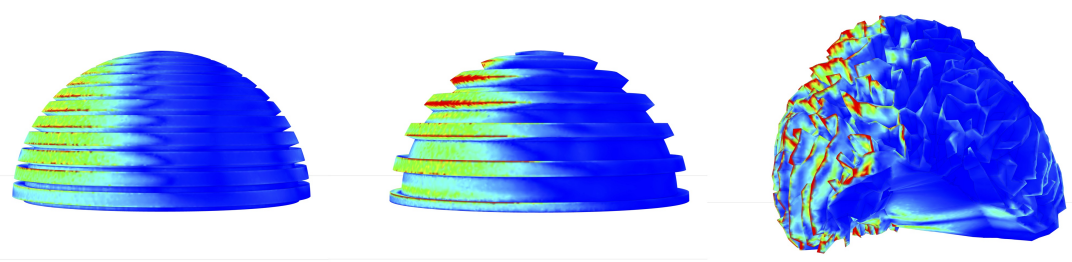

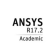

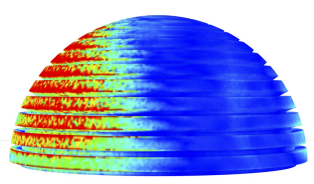

$\mathrm{w} / \mathrm{k}=0.5$

$\mathrm{w} / \mathrm{k}=2.0$
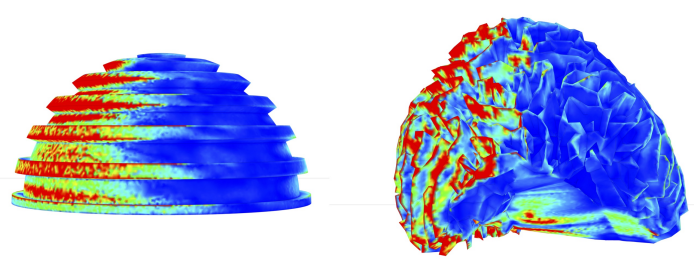

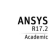
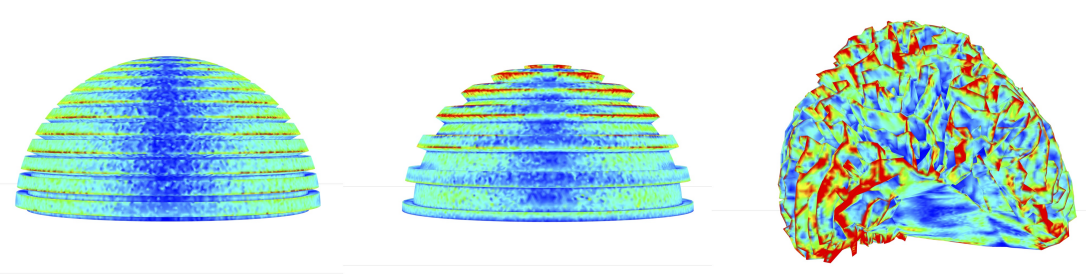

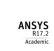
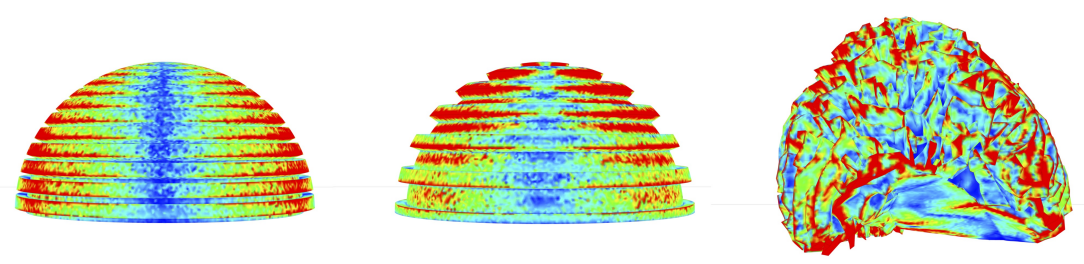

Coral

Figure 5.17: Surface plots of mean wall shear stress for unidirectional (top) and oscillatory (bottom) flows for low and high $R e$ regimes.

for unidirectional versus oscillatory flows - which suggests mass and heat transfer may also experience similar trade-offs. 

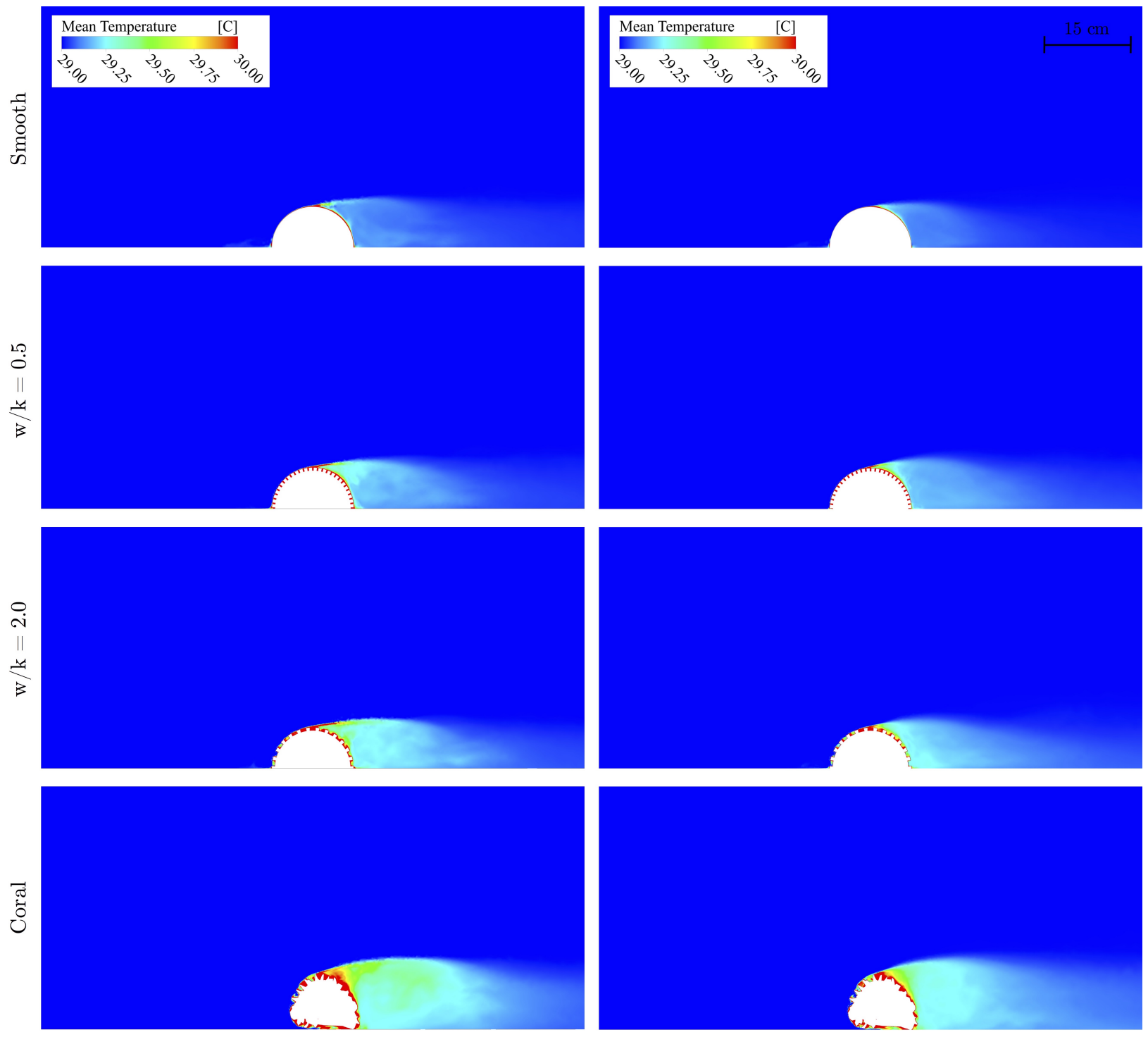

$R e=6,750$

$R e=20,250$

Figure 5.18: Time-averaged temperature fields for low (left) and high (right) Re unidirectional flow test cases.

\subsubsection{Results: Heat Transfer Characteristics}

Numerical model results for interfacial heat flux and convective heat transfer can be easily visualized through two-dimensional field plots of temperature distribution. Figure 5.18 shows mean temperature fields for high and low Re unidirectional flow past all four surface geometries. The background bulk fluid temperature and inlet fluid temperature are both $29^{\circ} \mathrm{C}$, while the surface temperature is held constant at $32^{\circ} \mathrm{C}$. The increases in thermal convection from the hemisphere surfaces correlate with increasing $w / k$ ratio, as indicated by the larger area and higher density of high-temperature colors. The exception is the coral skeleton, which has an approximate $w / k$ ratio equal to 1 , but because the roughness spacing is not radially symmetric like 

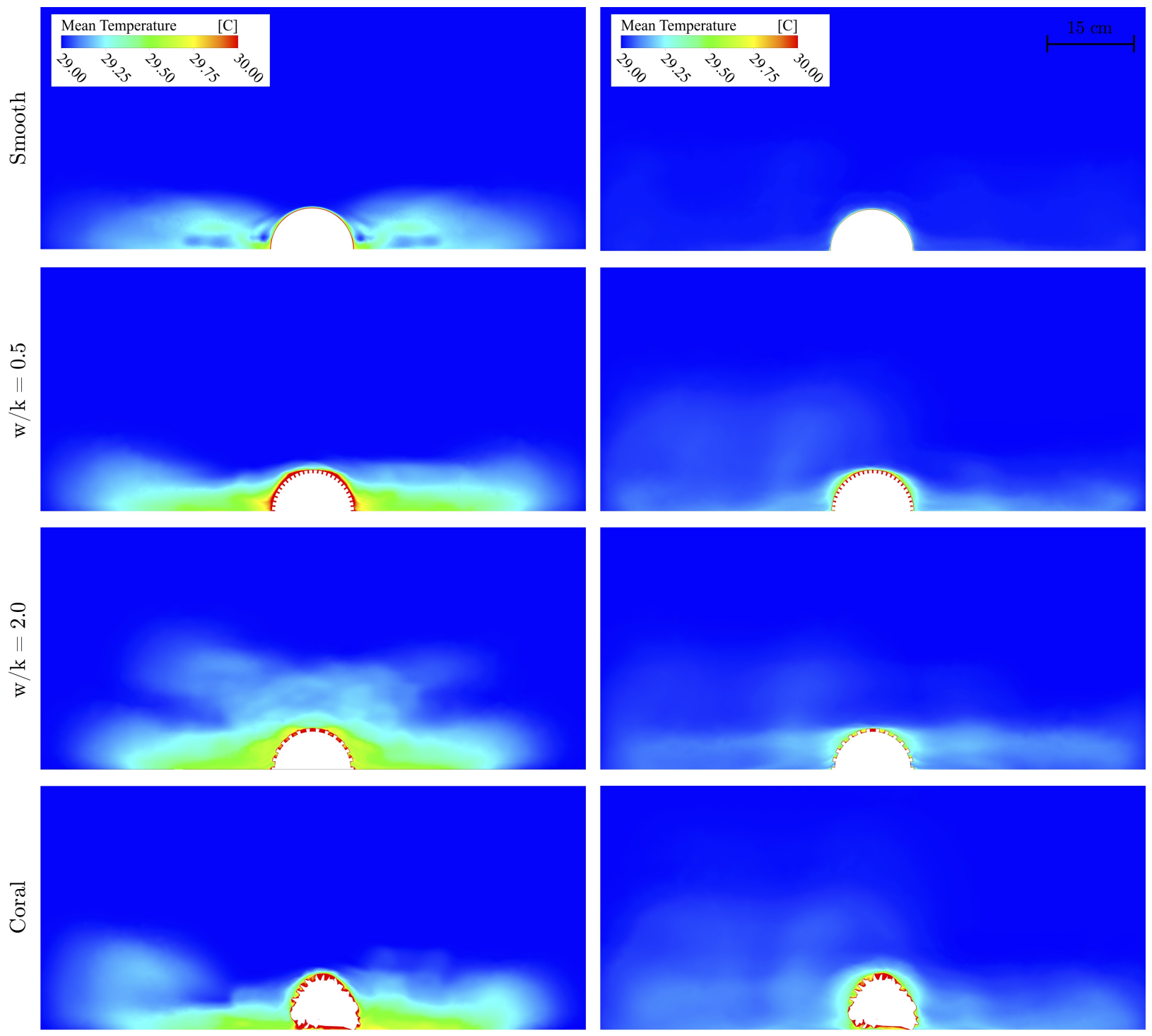

$$
R e=6,750
$$

$$
R e=20,250
$$

Figure 5.19: Time-averaged temperature fields for low (left) and high (right) Re oscillatory flow test cases.

the idealized models, the coral model possesses a significantly higher total surface area from which to shed thermal energy. The temperature distributions of the high Reynolds number flow are less intense compared to the low Reynolds flow because the higher fluid velocities advect more of the thermal energy downstream and the higher Reynolds shear stresses more effectively mix out thermal gradients. However, a similar pattern of increasing thermal field with increasing roughness is seen in these plots.

Mean temperature fields under oscillatory forcing are shown in figure 5.19. The same distribution patterns, i.e., larger, more intense temperature fields with increasing $w / k$ ratios and more diffuse temperature fields at higher Re, are seen in the oscillatory plots as in the unidirectional plots. Comparing the 

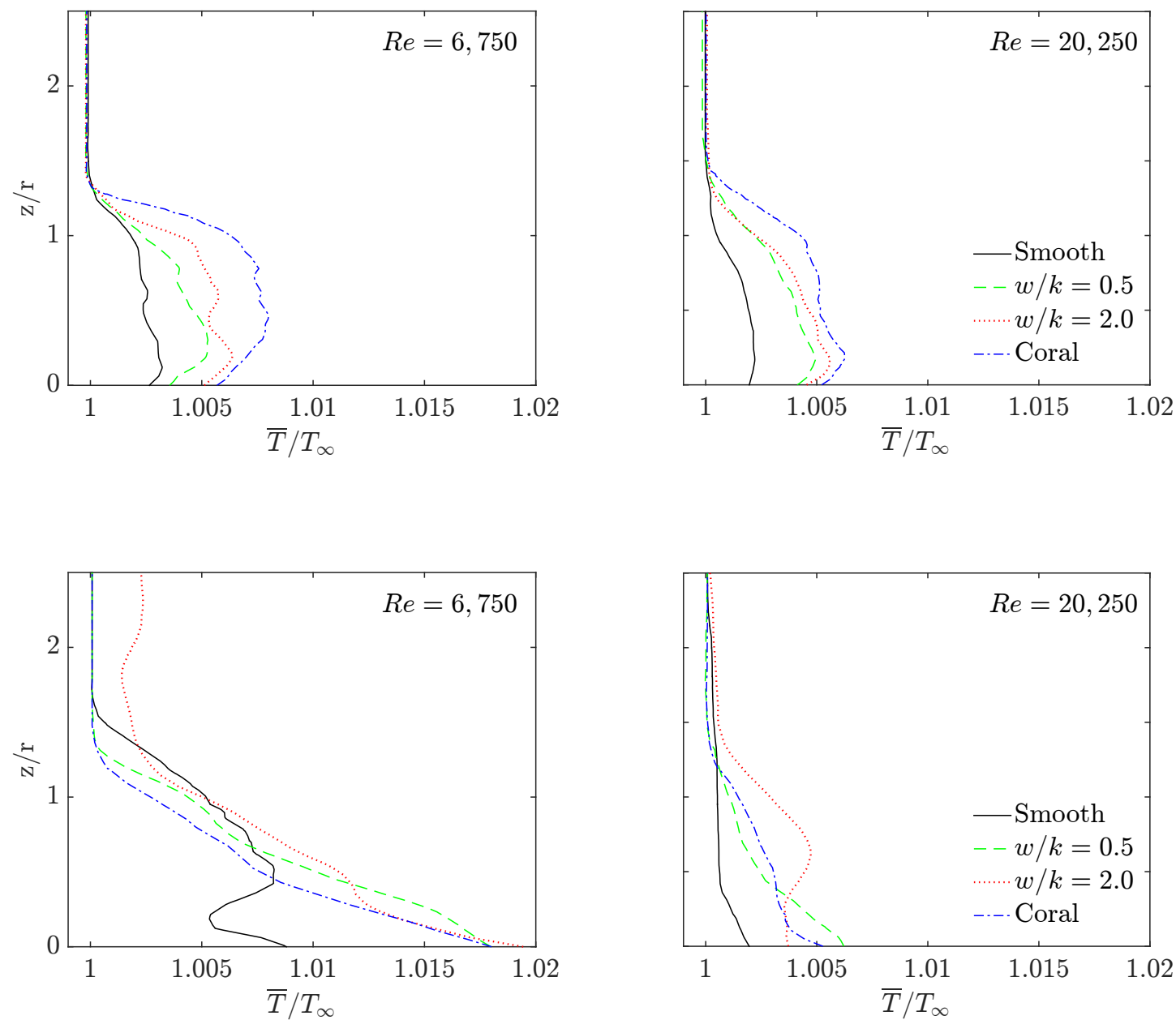

Figure 5.20: Vertical profiles of mean temperature $\bar{T}$ (normalized by background bulk fluid temperature $T_{\infty}$ ) for unidirectional (top) and oscillatory (bottom) flows at x-axis location one diameter downstream from trailing edge of model, i.e., $x / D=1$.

temperature distributions between unidirectional and oscillatory test cases for both low and high $R e$, it is evident that the oscillatory flow transfers higher levels of thermal energy from the hemisphere surfaces and that oscillatory motion confines the higher temperature fields to regions closer to the hemisphere models. Quantitive comparisons can be made by plotting vertical profiles of temperature distribution one model diameter downstream of the trailing edge. Figure 5.20 plots mean temperature normalized by bulk fluid temperature for all four surface geometries at low and high $R e$ under unidirectional and oscillatory forcing.

For unidirectional flows, the k-type model shows a temperature enhancement of approximately $1.5 \%$ and $3 \%$ over the d-type and smooth models, respectively. (The coral model is even greater at $3.5 \%$ and $5.5 \%$, respectively). For oscillatory flows, the low Re test case shows much higher temperature values for all 

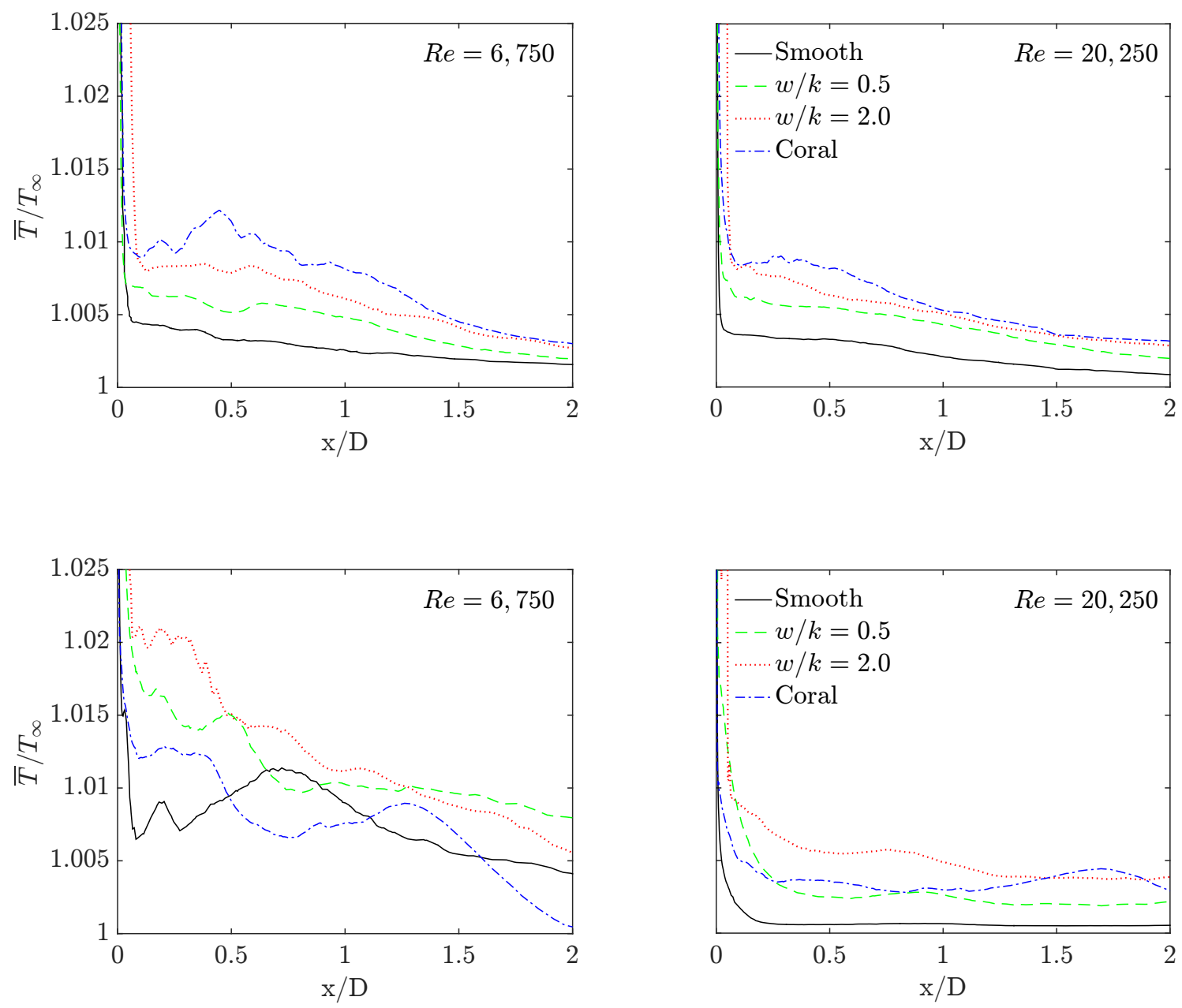

Figure 5.21: Horizontal profiles of mean temperature $\bar{T}$ (normalized by background bulk fluid temperature $T_{\infty}$ ) for unidirectional $(t o p)$ and oscillatory (bottom) flows. Profiles begin adjacent to model trailing edge and extend to end of computational domain. Profiles are taken at a vertical elevation equal to one-half model height, i.e., $z / r=0.5$.

models - on the order of a $5 \%$ increase - but the general pattern of k-type temperature enhancement over d-type remains, as it also does for the high Re oscillatory case. Quantification of downstream profiles can also be made by extracting horizontal profiles from the models' trailing edges to the end of the computational domain. Figure 5.21 plots profiles for low and high Re unidirectional and oscillatory flows. Profiles in the streamwise direction demonstrate that the enhancements seen in vertical profiles of figure 5.20 are sustained in an approximately linear fashion as one moves downstream from the models' trailing edges.

Close-ups of near-surface temperature fields are shown for each surface geometry and flow condition in figure 5.22. The colorbar scaling - ranging from a low of $29^{\circ} \mathrm{C}$ associated with the bulk fluid temperature to a high of $32^{\circ} \mathrm{C}$ associated with the model surface temperature - is different from the far-field plots, whose 

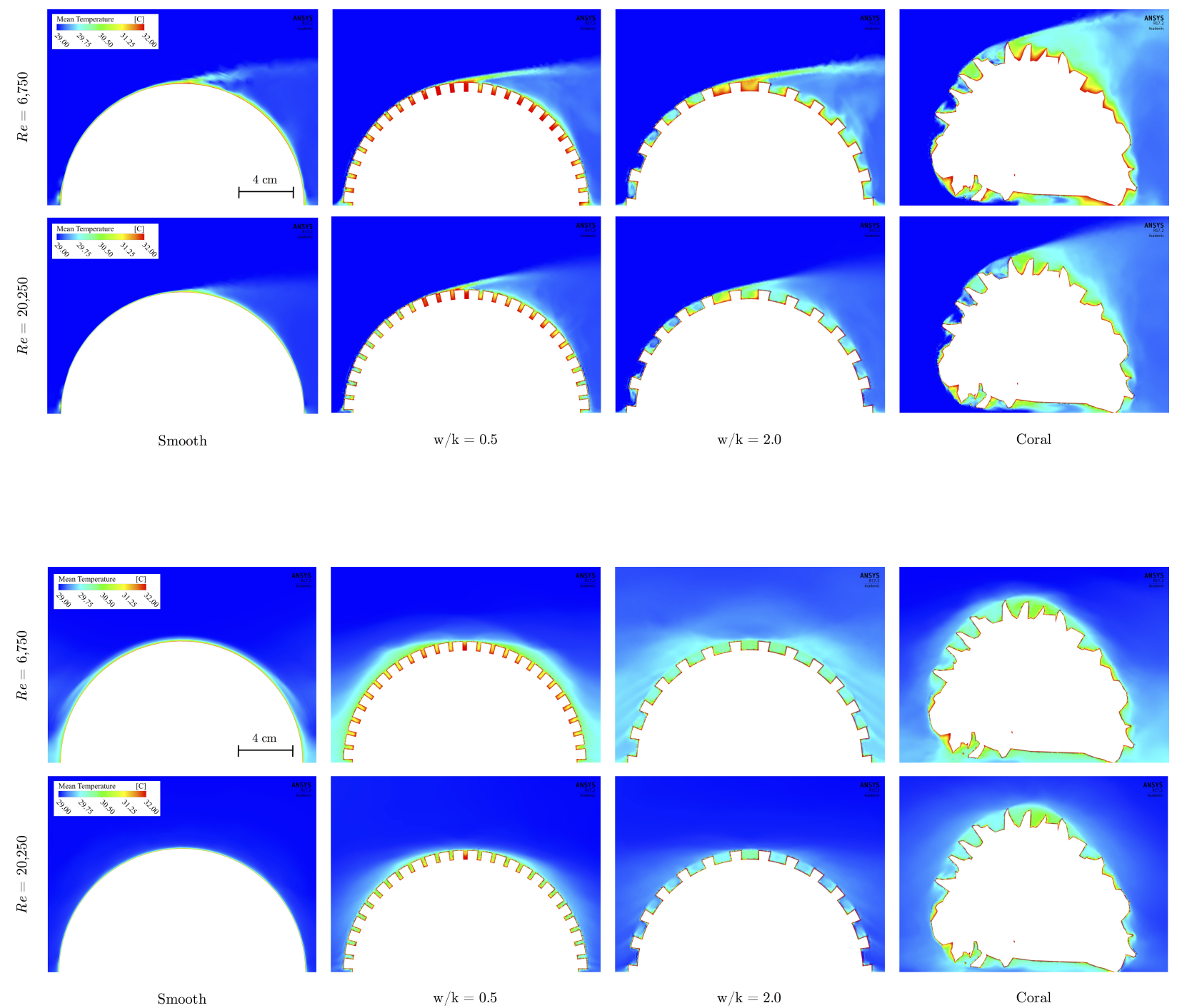

Figure 5.22: Near-surface close-up field plots of mean temperature $\bar{T}$ for unidirectional (top) and oscillatory (bottom) test cases for low and high Re regimes.

colorbar ranges were reduced to visualize more clearly the downstream temperature fields. Surface cavities filled entirely in red, for example, along the top of the d-type model in low unidirectional flow, indicate that the cavity water heated by the model surface is not being flushed out as effectively, and thereby inhibiting additional heat flux from the surface. By contrast, the k-type and coral model roughness cavities show much lighter temperature fields, which become increasingly flushed as Reynolds number increases and as oscillatory flow is experienced. The oscillatory test cases demonstrate that the narrow d-type cavities are much more effectively cleared of high temperature fluid compared to the unidirectional case and that increasing Reynolds number further enhances thermal flux away from the model surface.

Finally, surface plots of mean wall heat flux are shown in figure 5.23 for each surface geometry and flow condition. Similar to the wall shear plots in figure 5.17, the oscillatory test cases exhibit smaller peak 


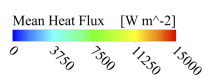

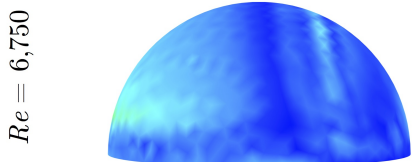

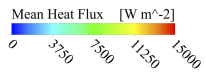

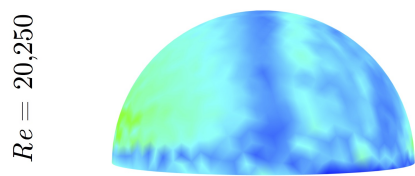

Smooth

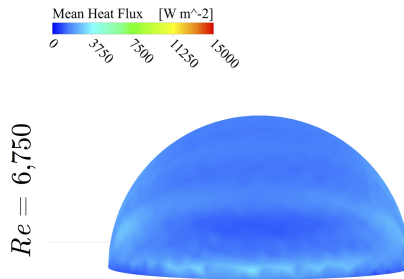

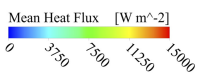

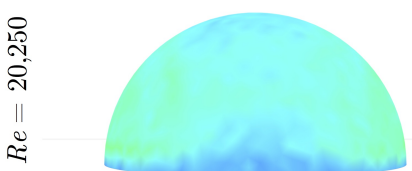

Smooth
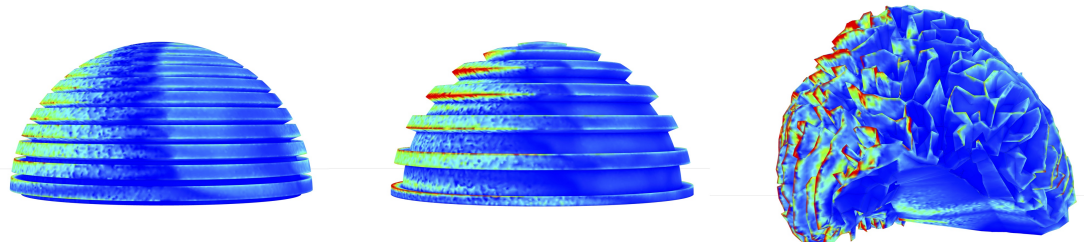

ANSYS

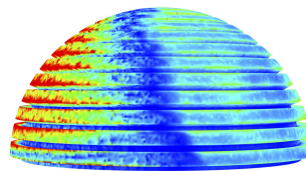

$\mathrm{w} / \mathrm{k}=0.5$

$\mathrm{w} / \mathrm{k}=2.0$
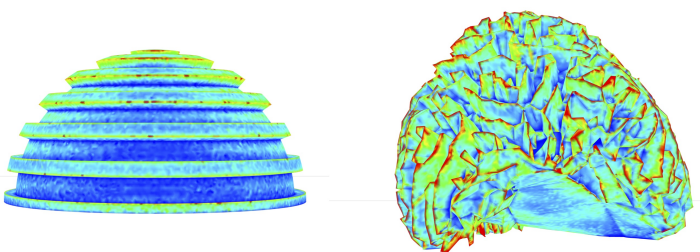

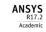
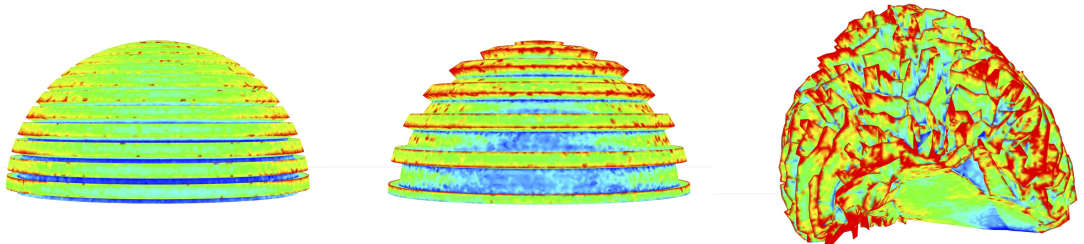

Coral

Figure 5.23: Surface plots of mean wall heat flux for unidirectional (top) and oscillatory (bottom) flows for low and high $R e$ regimes.

fluxes compared to their equivalent unidirectional cases, but the amount of surface area exhibiting nonnegligble heat flux is significantly increased. The roughness cavities for the k-type model show high levels of heat flux across all walls of the cavity, as was suggested by the vortical motion plotted in figure 5.15 and in the surface-normal profile plots of figure 5.16. Close inspection of the d-type roughness cavities show that 


\begin{tabular}{lccccc} 
Mean Wall Heat Flux, $\bar{q} \times 10^{3}\left(\mathrm{~W} \mathrm{~m}^{-2}\right)$ & & & & \\
\hline Flow Regime & $u_{r m s}\left(\mathrm{~cm} \mathrm{~s}^{-1}\right)$ & Smooth & $w / k=0.5$ & $w / k=2.0$ & Coral \\
\hline \hline Unidirectional & 5 & 1.78 & 1.32 & 2.14 & 2.58 \\
Oscillatory & 5 & 1.77 & 1.94 & 3.71 & 5.18 \\
Unidirectional & 15 & 3.51 & 3.79 & 5.50 & 6.63 \\
Oscillatory & 15 & 4.18 & 6.41 & 8.84 & 10.37 \\
\hline
\end{tabular}

\begin{tabular}{lccccc} 
Total Heat Flux, $q_{\text {tot }} \times 10^{2}(\mathrm{~W})$ & \multicolumn{1}{l}{} \\
\hline Flow Regime & $u_{r m s}\left(\mathrm{~cm} \mathrm{~s}^{-1}\right)$ & Smooth & $w / k=0.5$ & $w / k=2.0$ & Coral \\
\hline \hline Unidirectional & 5 & 0.51 & 0.81 & 0.95 & 2.05 \\
Oscillatory & 5 & 0.51 & 1.20 & 1.64 & 4.11 \\
Unidirectional & 15 & 1.00 & 2.33 & 2.43 & 5.27 \\
Oscillatory & 15 & 1.20 & 3.95 & 3.91 & 8.24 \\
\hline
\end{tabular}

\begin{tabular}{lccccc} 
Mean Heat Transfer Coefficient, $\bar{h} \times 10^{3}\left(\mathrm{~W} \mathrm{~m}^{-2} \mathrm{~K}^{-1}\right)$ & & & & \\
\hline Flow Regime & $u_{r m s}\left(\mathrm{~cm} \mathrm{~s}^{-1}\right)$ & Smooth & $w / k=0.5$ & $w / k=2.0$ & Coral \\
\hline \hline Unidirectional & 5 & 0.59 & 0.44 & 0.71 & 0.86 \\
Oscillatory & 5 & 0.59 & 0.65 & 1.24 & 1.73 \\
Unidirectional & 15 & 1.17 & 1.26 & 1.83 & 2.21 \\
Oscillatory & 15 & 1.39 & 2.14 & 2.95 & 3.46 \\
\hline
\end{tabular}

Table 5.4: Top: Surface area-averaged and time-mean wall heat flux values $\bar{q}$. Middle: Total amount of heat flux $q_{t o t}$ per unit time. Bottom: Mean heat transfer coefficient $\bar{h}$.

the bottom surfaces show negligible rates of heat transfer, but that the high Re conditions do drive heat flux farther down the sides of the cavity walls, as also implied by the surface normal velocity plots of figure 5.16. Comparisons to the wall shear stress plots in figure 5.17 indicate that areas of highest surface stress correlate with areas of greatest wall heat flux, as would be expected from the large velocity gradients that determine shear levels and define the heat transfer governing equations.

To quantify the amount of convective heat flux for flow past the model hemispheres, areal means of time-averaged wall heat flux were calculated for each surface geometry and flow condition. Mean wall heat flux values $\bar{q}$ are given in table 5.4 (top). These values were then multiplied by the appropriate model surface area (given in table 5.1) to produce the total amount of thermal energy fluxed from the model surface per unit time, given in table 5.4 (middle). Lastly, areal means of dimensionless heat transfer coefficient $\bar{h}$ were calculated according to equation 5.1 and are given in table 5.4 (bottom).

The general trend of the heat flux table is consistent with the previous figures above, i.e., greater heat transfer rates occur for larger Reynolds numbers (ranging from $2 \mathrm{x}$ to $3.3 \mathrm{x}$ enhancement) and for oscillatory, rather then unidirectional, flow (ranging from $1.2 \mathrm{x}$ to $2 \mathrm{x}$ enhancement). Rates of mean heat flux also increase as surface roughness increases, though the coral model, at $w / k \sim 1.0$, does not follow this pattern because of its highly convoluted surface. Two key exceptions to these trends should be noted. First, the average wall heat flux for unidirectional low Re flow past the smooth hemisphere is actually greater 
than for comparable oscillatory flow - a sharp contrast to all other unidirectional/oscillatory comparisons. Second, the total heat flux $q_{t o t}$, which takes into account the model surface area, mirrors the pattern of the mean heat flux $\bar{q}$ of increasing values with increasing roughness, except for the high Re oscillatory flow past the d-type hemisphere. Under these conditions, the penetration of flow into the narrow roughness cavities allows for the greater surface area of the d-type hemisphere to outperform (in terms of heat transfer) the greater roughness-generated turbulence of the k-type model. The implications of these roughness/surface area tradeoffs on individual hemisphere models and on downstream flow environments will be discussed below.

\subsection{Discussion}

\subsubsection{Boundary layer and wake hydrodynamics}

The objectives of the present study were twofold: first, to quantify and describe the role of surface roughness on the turbulence characteristics of the near-wake and downstream flow structure, and second, to analyze the effect of these roughness-generated flow differences on interfacial heat flux and far-field convective heat transfer. The hydrodynamic results here show that the presence of surface roughness on a wall-mounted hemisphere alters the structure of mean velocity and turbulence characteristics, and that the degree of alteration depends on roughness type, Reynolds number, and unidirectional versus oscillatory flow conditions. At low Reynolds numbers for both unidirectional and oscillatory flows, the mean flow characteristics of the d-type roughness more closely resemble that of the smooth hemisphere than the k-type roughness, especially with regard to recirculation size and strength and mean velocity field (see figures 5.8 through 5.11). Whereas at high Reynolds number, the d-type roughness begins to exhibit hydrodynamic characteristics more similar to the k-type roughness than the smooth hemisphere, with much larger recirculation lengths and negative velocities.

The likely physical mechanism that drives the mean field dynamics between these roughness types and Re regimes is the relative influence of frictional drag versus pressure drag induced by the roughness elements and the amount of momentum this imparts into the boundary layer flow. Direct numerical simulations of flat-plate roughness have shown that the historical classification of d-type roughness as $w / k<1$ is better described by high ratios of frictional $\operatorname{drag} C_{f}$ to pressure drag $P_{d}$, while k-type roughness exhibits lower ratios 38. The large-scale curvature of the hemisphere body, however, alters the orientation of the roughness elements in relation to the incoming flow. In contrast to flat-plate d-type roughness, this curvature allows boundary layer flow to enter the d-type roughness cavities, as demonstrated by figure 5.16, and 
thereby increase the amount of pressure drag from individual roughness elements. This mechanism underlying the transition of d-type roughness behavior with Reynolds number can be confirmed by calculating the average surface pressure along a line integral for the front half of each hemisphere model. As predicted, at low Reynolds number $\bar{P}_{d}$ for the d-type roughness measures $0.30 \mathrm{~Pa}$ and is closer to $\overline{P_{d}}$ for the smooth model, $0.35 \mathrm{~Pa}$, than for the k-type model, 0.43 Pa. In fact, the narrow d-type roughness cavities absorb some of the high pressure generated by leading edge streamline compression resulting in lower within-cavity pressure and a smaller total $\bar{P}_{d}$ compared to the smooth model. At high Reynolds number, the relationship is reversed, with the d-type and k-type pressure drags measuring $2.73 \mathrm{~Pa}$ and $3.72 \mathrm{~Pa}$, respectively, while the smooth model is only 1.96 Pa. (For completeness, the coral model generates the highest pressure drag among all models at $0.52 \mathrm{~Pa}$ and $4.36 \mathrm{~Pa}$ for low and high Re, respectively.)

With regard to turbulence characteristics, flow past a bluff body is comprised of a superposition of three key physical processes - the primary wake instability, the separating shear layer, and the surface boundary layer. The largest scale eddies are generated by von Kármán vortex shedding induced by unsteady downstream pressure fields, which scale with the size of the bluff body [159. Kelvin-Helmholtz instabilities in the shear layer produce much smaller turbulent fluctuations, which scale with the strength of the shear layer 131 . And the smallest scale turbulent eddies derive from flow interaction with the hemisphere surface, either through the development of a turbulent boundary layer at high enough $R e$ or from vortex shedding from individual roughness elements. Since the turbulence dissipation rate $\epsilon$ must match the rate of turbulent kinetic energy input from these three processes, and since the most energetic eddies derive from the same model length scale, it is expected that $\epsilon$ should be similar among all models within the same flow regime. The results in table 5.3 show this to be valid, with each particular flow condition generating dissipation rates of approximately the same magnitude across all model types.

Though the large-scale shedding mechanism dominates the TKE input (and subsequent dissipation rate), the smaller scale roughness-induced turbulent eddies are responsible for the differences in Reynolds shear stresses among model types (see figure 5.14), especially for low Re flows, in which the shear layer strength is smaller and associated Kelvin-Helmholtz instabilities contribute less to $\overline{u^{\prime} w^{\prime}}$. The location and strength of eddy vortices can be identified using the Q-Criterion, which is defined as the second invariant of the velocity gradient tensor, i.e., $Q=-\frac{1}{2} A_{m n} A_{n m}$, (which, after manipulation of tensor algebra, yields the difference between the vorticity tensor and strain rate tensor) [160. Figure 5.24 shows the distribution of eddies along the leading edge roughness elements at low $R e$ unidirectional flow. The peak values of eddy strength for the k-type roughness are approximately $40 \%$ greater than the d-type roughness $\left(69.1 \mathrm{~s}^{-2}\right.$ versus $48.5 \mathrm{~s}^{-2}$, respectively) and $300 \%$ greater than the smooth model $\left(23.0 \mathrm{~s}^{-2}\right)$. The magnitude of this pattern of increasing eddy strength with increasing roughness matches almost exactly the respective downstream 


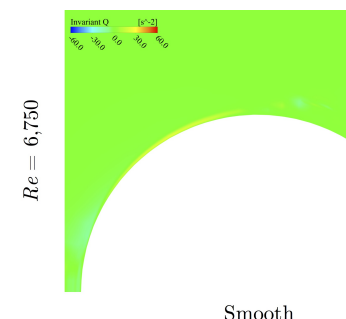

Figure 5.24: Instantaneous eddy vorticity fields for unidirectional low Reynolds number flow, as identified by the Q-Criterion.

Reynolds shear stress levels (see figure 5.14). The larger number of elements that comprise the d-type roughness allow it to "make up" for lower peak magnitudes and narrow the downstream shear stress gap to only $\sim 20 \%$. At high $R e$ the same pattern of roughness type and eddy strength remains (data not shown), but now, the shear layer instabilities contribute more to the stress levels and nearly wash out the high-frequency roughness eddy contributions, though some distinction still persists.

The oscillatory flow regimes exhibit significantly altered dynamics and are driven by different underlying physical mechanisms. Turbulence dissipation rates one model diameter downstream are much lower in comparison to equivalent unidirectional flow because the relatively short wave period does not allow enough time for either the von Kármán vortex shedding mechanism to set up or for the Kelvin-Helmholtz instabilities to propagate (and because the absence of a background mean current prevents the turbulence characteristics from being advected downstream as in the unidirectional case). Rather, the majority of vortex production comes from eddy roll-offs generated during flow reversal. As velocity slows leading to reversal, it encounters a large adverse pressure gradient, which induces shear instabilities that grow as the flow reverses and begins to interact with the model surface to create additional areas of unstable pressure gradients, similar to the dynamics seen in experiments of oscillatory flow over sand roughness 74 . Figure 5.25 illustrates this process by showing a time series of streamwise velocity, pressure field, and Q-Criterion for one-half of a wave cycle at high Reynolds number for the k-type model. At $t=0$, horizontal velocity $u(t)$ is at a maximum, and the flow and pressure fields resemble the unidirectional case with vortices occupying the leading edge roughness cavities. (The downstream eddies are residual from the previous wave cycle). In the decelerating phase at $t=\pi / 4$, the strength of the leading edge vortices weakens and recirculating flow on the downstream edge is enhanced. At the point of reversal at $t=\pi / 2$, the inertia of the downstream recirculating flow carries it along the top of the model where disturbance in the pressure field have developed, but the Q-Criterion strength of existing eddies is quite small. After flow reversal at $t=3 \pi / 4$, strong negative pressure gradients near the model top destabilize the flow and induces intense eddy vortices at this location. Finally, at $t=\pi$, the horizontal velocity flow field is fully reversed with large vortices occupying the (previously) downstream 


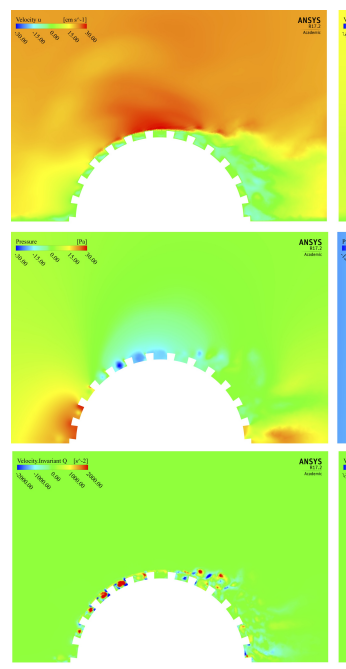

$t=0$
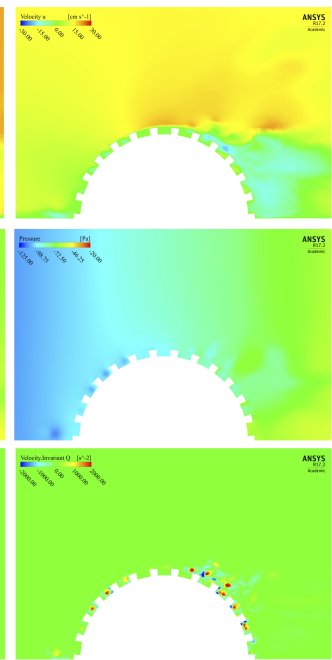

$t=\pi / 4$

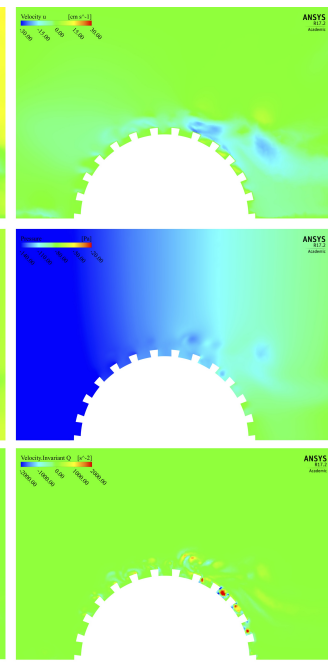

$t=\pi / 2$
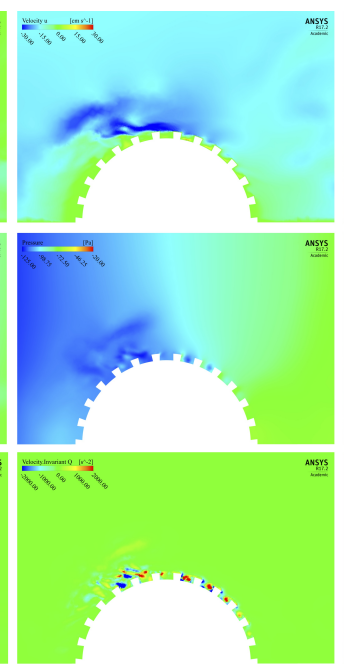

$t=3 \pi / 4$
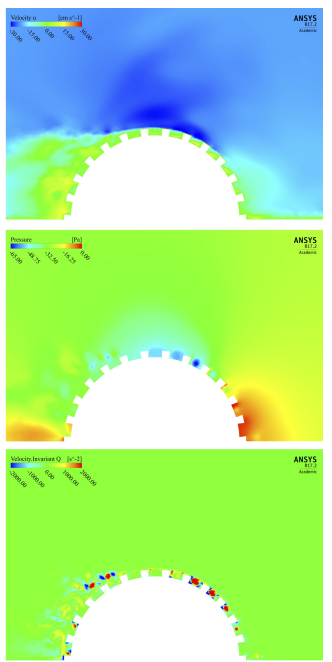

$t=\pi$

Figure 5.25: Time series of streamwise velocity (top), pressure (middle), and Q-Criterion (bottom) across one-half of a wave cycle for high $R e$ oscillatory flow past the k-type roughness model.

roughness cavities and the (newly) downstream edge eddies being advected away from the model. The roll-off eddies generated in this way produce mean Reynolds stresses that are an order of magnitude larger than for comparable unidirectional flows (note the y-axis scale in figure 5.14) and should act to homogenize both velocity and thermal/scalar gradients more efficiently.

\subsubsection{Surface roughness and flow effects on convective heat transfer}

Results from the convective heat transfer model show that the interfacial heat flux is controlled to varying degrees by each of the three parameters investigated: Reynolds number, surface roughness type, and flow regime, as well as interacting effects among the three controls. The most direct and most significant impact results from increasing Reynolds number, where a 3 -fold increase in Re produces a $2 \mathrm{x}$ to $3.3 \mathrm{x}$ enhancement in mean heat flux. The second most important control on $\bar{q}$ is the type of surface roughness, where the k-type roughness demonstrates an enhancement in mean heat flux of 1.2-2.1x compared to the smooth and d-type models. Last, the presence of oscillatory flow enhances mean heat flux by 1.2-2.0x compared to equivalent unidirectional flow (with the exception of the low Re flow past the smooth model, where $\bar{q}$ is nearly equal between the two regimes).

The dynamics of Reynolds number control are well established - e.g., thinning of the thermal boundary layer, enhanced surface shear, greater fluid advection - and the impact of $R e$ on the calculated heat flux for the unidirectional flow cases past the smooth model compares well to the empirically predicted heat transfer correlation given in equation 5.6 The numerical model over-predicts $\bar{h}$ by approximately $20 \%$ for each case (594 vs. $499 \mathrm{~W} \mathrm{~m}^{-2} \mathrm{~K}^{-1}$ and 1170 vs. $924 \mathrm{~W} \mathrm{~m}^{-2} \mathrm{~K}^{-1}$, respectively), but the correlation equation 


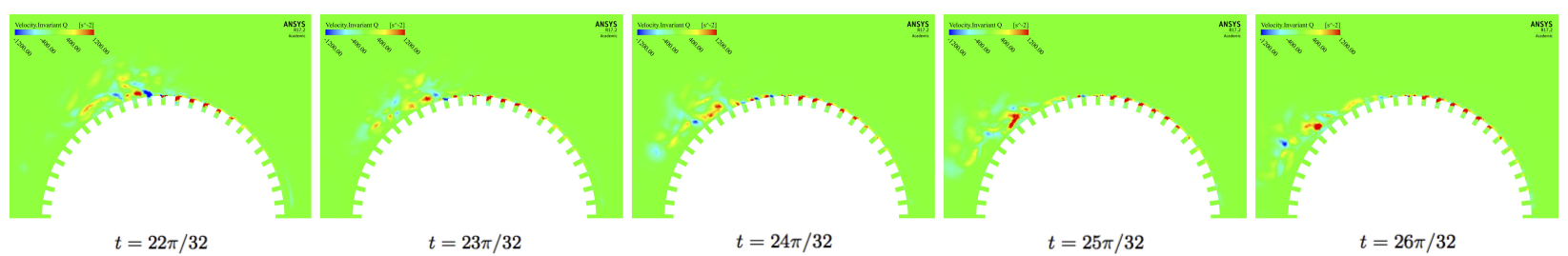

Figure 5.26: Time series of turbulent eddy distribution, as measured by Q-Criterion, for d-type model during accelerating phase after flow reversal, i.e., after $t=\pi / s$, under high $R e$ conditions.

has been shown to produce uncertainties of up to $20 \%$ and was derived for spheres rather than hemispheres 152 .

Enhancement of heat flux resulting from surface roughness is governed by the hydrodynamic mechanisms described above, in particular by the production of high vorticity eddies within roughness cavities, which create steep velocity gradients and enhance surface shear, and by the increase in pressure drag associated with greater roughness. Skin friction drag actually decreases for the d-type roughness since much of the roughness cavity surfaces are sheltered, but the surface curvature of the model - in conjunction with the presence of roughness elements - means the pressure drag is the dominant term, as is the case for all models (ranging between 90-96\% of total drag $C_{d}$ ). As described above, at low Re for unidirectional flow, the d-type roughness has the smallest total drag, followed by the smooth model and the k-type model, which corresponds to the order of heat transfer coefficient magnitude. By contrast, at high $R e$, total $C_{d}$ increases monotonically with surface roughness, and $\bar{h}$ follows accordingly. In each case, $\bar{h}$ is determined by the total model drag $C_{d}$-a correlation well documented in the literature 151. With the exception of low $R e$ flow past the d-type model, surface roughness - as measure by $w / k$ ratios - acts to increase $C_{d}$ and thereby enhance $\bar{h}$ (though only up to some optimal ratio, which for flat plates was shown to be $w / k=7$ as demonstrated by DNS data from Leonardi et al. (2003) [138]).

For oscillatory flow conditions, the increase in heat transfer coefficients results from a combination of several physical mechanisms. First, the alternating positive and negative horizontal velocities allow for a greater amount of model surface area to experience high wall shear stresses, as seen in figure 5.17. Next, at the point of flow reversal, large negative pressure fields are induced on the (previously) leading edge, which can be seen in figure 5.25 at $t=\pi / 2$. This negative pressure acts to "suction out" high temperature fluid from the roughness cavities, which, upon close inspection of the velocity field in figure 5.25, shows large, though diffuse, negative velocities at the model's left edge. Lastly, the roll-off eddies described above advect downward to draw additional thermal energy out of the upper roughness cavities, but which also act to cut off the high temperature fluid that had been suctioned from the surface and advect it away from the model. Figure 5.26 shows a time series of this eddy advection - as measured by Q-Criterion — that occurs in close 


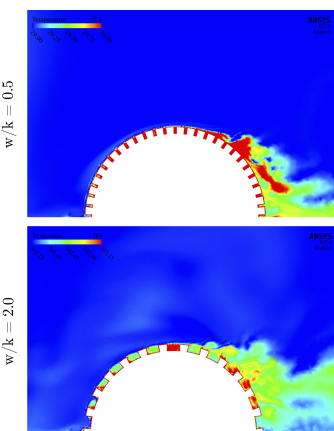

$t=0$
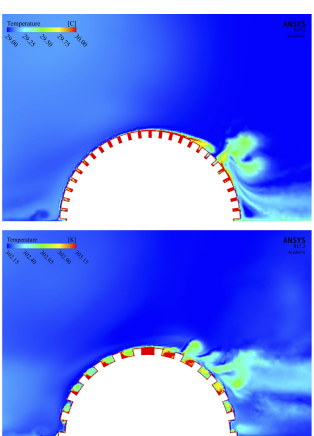

$t=\pi / 4$
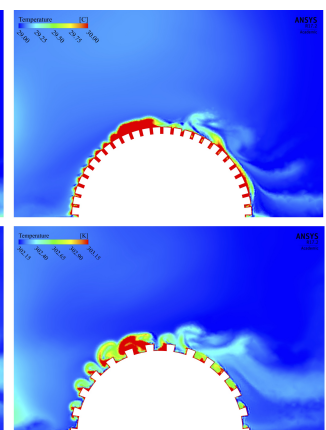

$t=\pi / 2$
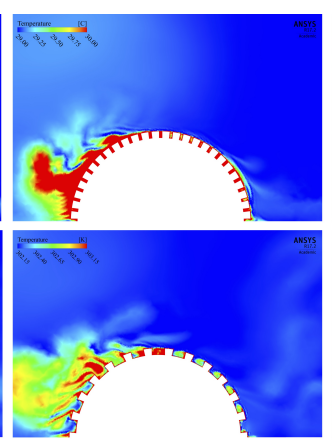

$t=3 \pi / 4$

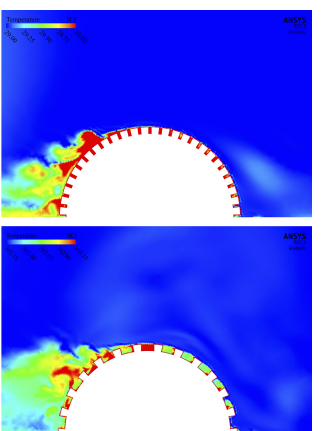

$t=\pi$

Figure 5.27: Time series of temperature fields for d-type (top) and k-type (bottom) models across one-half wave cycle of high $R e$ oscillatory flow.

succession around the $t=3 \pi / 4$ wave phase. This entire process is illustrated in figure 5.27, which shows a time series of temperature fields for both d-type and k-type models demonstrating suction at $t=\pi / 2$, eddy roll-off at $t=3 \pi / 4$, and high-temperature fluid advection at $t=\pi$. Unlike previous studies of oscillatory flow through canopies or branching structures, in which the time-varying flow field has been shown to enhance mass flux by creating pressure fields that drive higher in-canopy flow speeds [64, 153], the rough bluff bodies investigated here exhibit significantly different physics, yet experience a similar magnitude of heat transfer enhancement resulting from oscillatory flow.

\subsubsection{Biological and ecosystem implications of heat and mass transfer}

The rate of heat/mass transfer across the coral-water interface has been shown to play a role in a number of processes critical to the biological success of corals, such as driving rates of photosynthesis [31, directing structural growth [161, and mitigating heat-induced bleaching 162. The high rugosity of reef systems and the maximization of coral surface area available for these biophysical process has been suggested as a key explanation for the so-called "Darwin's Paradox", which attempts to reconcile the high productivity of reef ecosystems with the relative paucity of nutrients found in tropical aquatic habitats of corals. To this end, experimental work on gypsum dissolution of rectangular forms with mm- and cm-scale roughness by Falter et al. (2005) 37] demonstrated a positive linear relationship between rugosity, i.e., the ratio of total surface area-to-planar projected area (given in table 5.1) and mass transfer coefficient $\bar{h}_{m}$. However, the heat transfer results here indicate that such a relationship is not present and is directly contradicted by the higher values of $\bar{h}$ for the k-type roughness, despite its smaller rugosity and lower total surface area. Falter et al. suggest their experimental design allowed for all surfaces of the model to experience comparable flow conditions, which the mean velocity plots show not to be the case here, and which does not account for the differing mechanisms of turbulence-driven heat flux resulting from different roughness types and specific flow 
conditions.

Measurements of mass transfer coefficients for nutrients taken over coral reef flats by Bilger and Atkinson (1992) [27], by contrast, suggest rugosity is not a key determinant of mass flux and that the enhancement in $\bar{h}_{m}$ is $\sim 40 \mathrm{x}$ larger than would be expected from engineering relationships developed for flat-plate roughness [163. The authors suggest a variety of mechanisms that may be responsible for the unexpectedly high flux, such as wave surge, higher surface areas resulting from the fractal nature of reef organisms, and active ciliary pumping at the coral surface, which recent work has indeed shown to enhance mass flux 4 -fold 25]. The results of the current study demonstrate that using convection coefficients derived from unidirectional laboratory flow past flat-plate roughness as an analogue for wavy flow past reef structures fails to account for many of the additional physical dynamics that enhance heat transfer. Using the heat transfer correlation equations developed for flat-plate roughness by Webb et al. (1971) (the same as those employed by Bilger and Atkinson) [140, the low Reynolds number unidirectional flow over k-type roughness investigated in this study produces an estimated flat-plate $\bar{h}$ of $\sim 290 \mathrm{Wm}^{-2} \mathrm{~K}^{-1}$. The equivalent flow conditions for the k-type hemisphere produce a mean heat transfer coefficient nearly $2.5 \mathrm{x}$ larger $\left(\sim 714 \mathrm{Wm}^{-2} \mathrm{~K}^{-1}\right)$, which is a similar magnitude of heat transfer enhancement that is seen for smooth hemispheres compared to smooth plates using equations 5.6 and 5.4 above, respectively. Thus, the combination of ciliary action (4x enhancement), oscillatory fluid motion (2x enhancement), and hemisphere dynamics (2.5x enhancement) can account for roughly a $20 \mathrm{x}$ enhancement over predicted rough flat-plate convection coefficients.

Representing the entire reef as a rough flat plate may be useful for reef-scale parameterizations of terms like friction velocity $u_{*}$, drag coefficient $C_{d}$, or bottom friction $C_{f}$ in order to describe the meter-scale bottom boundary layer. Such descriptions of reef systems have been shown to not depend on the smallscale features of individual reef components 95 and have been successfully employed to estimate large-scale impacts such as wave dissipation 23] or whole-reef nutrient uptake 22. However, for an individual coralwhose health is critical to sustaining the entire reef ecosystem - the results presented here demonstrate that the hydrodynamic and heat transfer dynamics of the fine-scale near-surface boundary layer, embedded within the larger bottom boundary layer, depend heavily on small-scale surface features and individual coral topography. Spatial variation of wall shear and mass transfer rates have been shown to drive patterns of coral morphogenesis, where tissue aggregation is controlled by the rate of nutrient accumulation [14, 161, and thus, the hydrodynamic conditions of a particular coral habitat may apply selective pressure on the morphology best suited to that environment. As a colonial organism, though, with the ability to transport transport nutrients internally throughout an entire colony of polyps, an equally important consideration for coral health - in addition to spatial variability of local flux - is total mass transfer across the entire surface 
area. As such, the optimal surface morphology to drive maximum heat flux $q_{\text {tot }}$ depends on the specific combination of flow speed and oscillatory condition, as seen in table 5.4 and is result of the specific physics generated by the dynamic relationship between flow regime and roughness type.

In addition to describing the hydrodynamic and heat transfer impacts on individual corals, the numerical results in this study provide a characterization of downstream turbulence, mixing, and heat/mass flux that affects the bio-physical environment of other nearby reef structures and organisms. Field measurements of the flow environment behind a single coral bommie made by Hench and Rosman (2013) indicate that the wake structure for low frequency $(<0.03 \mathrm{~Hz})$ wavy flow creates a similar effect to unidirectional flow, which shelters organisms from high drag forces, reduces mean flow speeds, and enhances turbulent fluctuations and vertical mixing [123]. The simulation results for the unidirectional case here are consistent with these findings and also demonstrate that the nature of the surface roughness of the upstream obstruction significantly alters the hydrodynamic and scalar wake conditions. The variability within these far-field flow conditions creates an array of niche environments in which reef organisms can exploit specific conditions and increase habitat biodiversity. High frequency flows like the oscillatory cases investigated in this study, by contrast, generate a small flow excursion length $A_{\infty}^{\text {rms }}$ relative to the coral diameter $d$ (for high Re regime here, $A_{\infty}^{\text {rms }}$ is only $88 \%$ of $d$ ), which prevents the establishment of a persistent wake structure. Nevertheless, oscillatory flow enhances flux from the coral surface compared to an equivalent unidirectional flow, and as the temperature fields in figure 5.19 illustrate, also produces a range of potentially exploitable niche conditions, which are differentially affected by surface roughness. Thus, the wave period of the oscillatory flow establishes whether drag forces dominate (i.e., at low frequencies) or inertial forces dominate (i.e., at high frequencies). This interaction of flow speed, wave period, and surface roughness across a range of spatial and temporal scales in the natural environment creates a dynamic flow and resource landscape that has profound consequences on the bio-physical processes of surrounding reef organisms.

\subsection{Conclusions}

The major research question addressed in this chapter is how does surface roughness affect hydrodynamics and heat transfer for flow past a wall-mounted hemisphere. The implication of this question is that trade-offs exist between the advantage of using broadly spaced roughness to generate turbulence for enhancement of heat transfer versus the advantage of using densely spaced roughness to increase the amount of total surface area for enhancement of heat transfer. The results demonstrate that the preferred $w / k$ roughness ratio needed to optimize these trade-offs depends on the specific flow conditions, in particular the flow speed and whether the flow is unidirectional or oscillatory. Moving beyond the ideal flat-plate roughness ratio of 
$w / k \sim 7-8$ as demonstrated by Leonardi et al. (2003) and Ryu et al. (2007) 138, 151], the conclusions in this study offer new and interesting contributions to the literature and provide novel insights into the physical mechanisms and resulting impacts of the complex interaction between flow speed, steadiness of flow, and the nature of surface roughness. Ultimately, the results suggest potential new avenues of research within both the engineering flow and heat transfer literature, as well as the coral environmental fluid dynamics literature. The results of this dissertation aim to help the scientific community achieve a better understanding of not only fundamental physics in turbulence and fluid dynamics, but importantly, offer new insights into the resilience and sustainability of corals and the efforts we can take to promote their continued survival and future success. 


\section{Chapter 6}

\section{Conclusions}

The major results of this dissertation show how specific alterations to a coral surface impact the characteristics of boundary layer flow and affect the flux of mass and thermal scalars across the coral-water interface. In particular, Chapter 3 demonstrates that the addition of turf algae shifts the turbulence regime of the boundary layer from a wall-bounded inertial layer to a plane-mixing layer, which enhances turbulent mixing above the algal canopy but simultaneously reduces mass flux at the coral surface. Chapters 4 and 5 illustrate the profound hydrodynamic consequences resulting from variations in surface roughness and the presence of oscillatory flow, in which k-type roughness increases turbulence production and heat transfer, while oscillatory flow shifts the underlying physics and introduces new trade-offs in surface area-to-roughness optimization that favor d-type roughness under wavy, high speed flow conditions. Below, I situate the present results within the larger context of existing literature on coral biology and engineering roughness and describe the significant contributions of this research in extending our current understanding.

\subsection{Relevance to Current Research in Coral Biology}

Massive Scleractinian corals exhibit a wide range of surface morphologies in nature, from smooth, lightly dimpled surfaces to broad ridges with and without grooves to sharp, fin-type roughness (e.g., figure 6.1). The results presented in this dissertation demonstrate that specific variations in morphology, in particular the spacing between roughness elements, can drive significant differences in rates of heat (and mass) transfer. Though much inter-specific variation is determined by genetic differences 164, 165, long-term environmental conditions, such as the flow regimes investigated here, can create selective evolutionary pressures that optimize a particular surface topography for a dominant flow environment. At much shorter time scales, the phenotypic plasticity seen in intra-specific morphological variation has been shown to be a response to 

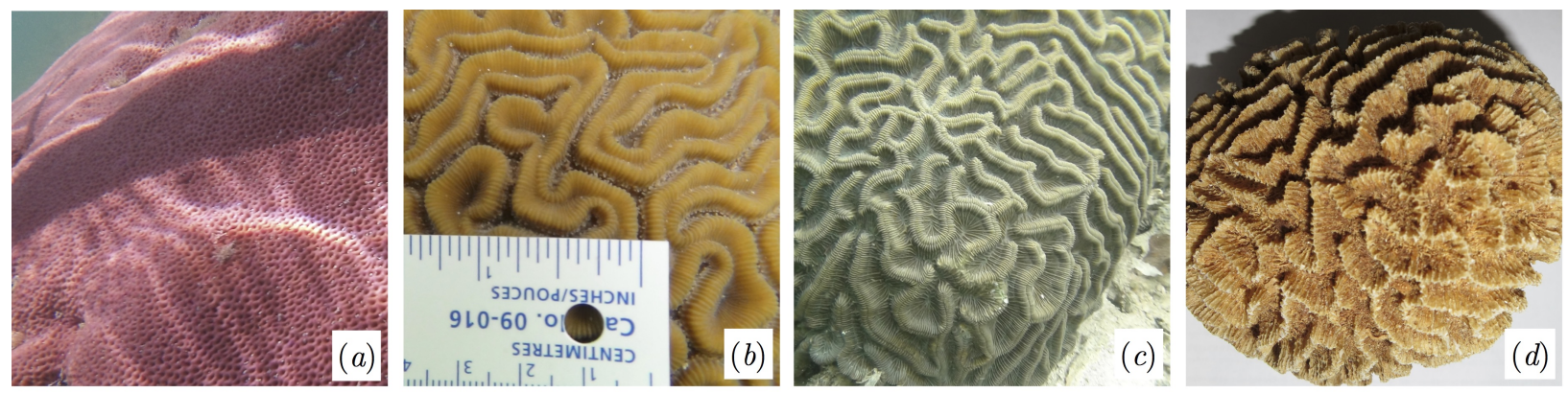

Figure 6.1: Photographs illustrating variation in Scleractinian surface morphology. (a) Siderastrea siderea. (b) Diploria labyrinthiformis. (c) Colpophyllia natans. (d) genus Platygyra, species-level identification unavailable.

environmental gradients (e.g., light, nutrient/prey availability, temperature), which are often controlled by local hydrodynamic conditions. Gradients in coral size have been shown to correlate with increasing wave and tide conditions [134, and faster flows have been shown to promote this distribution by generating denser carbonate structures, raising zooxanthellae counts, and improving reproductive output [166]. Since these coral growth and survival processes are driven by scalar fluxes at the surface, the results presented in Chapter 5 suggest that the enhanced heat transfer rates shown to result from rougher surfaces may expand the environmental conditions under which they can exist. For example, the heat transfer coefficient for the k-type model at low oscillatory flow is nearly equal to that of the smooth model at high oscillatory flow (1.24 versus $1.39 \mathrm{~W} \mathrm{~m}^{-2} \mathrm{~K}^{-1}$, respectively), which implies the rough model may be able to endure much lower energy flow environments than the smooth model, yet still benefit from comparable rates of heat and mass exchange.

Given flow as a key abiotic factor, then, in controlling coral productivity and directing growth, recent efforts were undertaken to quantify directly the rates of mass transfer subject to various flow and morphological conditions using primarily field/mesocosm measurements and laboratory gypsum dissolution methods. A direct relationship was demonstrated between flow speed and increased nutrient uptake over reefs in the field 28,167 . In the lab, the addition of oscillatory flow or roughness - either as branching structures or rubble resembling bluff bodies — was shown to enhance mass transfer up to 3-fold 37, 64, 153 and 9-fold [37, 91], respectively, compared to comparable unidirectional flow or smooth surfaces. The findings here are consistent with the existing literature, as well as with long-established convection correlations in the engineering literature for smooth spheres, as was demonstrated in Chapter 5. However, the simulations offer new insights into the combined effects of these variables, by demonstrating an important trade-off that occurs to alter the optimization strategy of sacrificing surface area in order to enhance turbulence generation. Under oscillatory high-speed flows, the benefits of greater surface area from narrowly spaced roughness outweigh the benefits of enhancing turbulence from broadly spaced roughness in the effort to maximize heat transfer. 
Such environmentally dependent trade-offs are likely to affect patterns of coral habitat distribution, but also impact differential survival rates during stress events and subsequent recovery rates, as has been documented for post-bleaching zooxanthellae recovery [135, 168].

Further, by investigating the specific physical mechanisms that drive this trade-off-as was done through a half-wave cycle in Chapter 5-I also show that the underlying physics differs from that which drives oscillatory flow mass flux enhancement for branching corals. In the case of branching canopies, oscillatory flow generates large pressure gradients between coral branches which drive higher in-canopy flow speeds and result in enhanced mass transfer 64,153 . By contrast, the hemispherical coral models here experience a shift in the relative importance of inertially driven flow, which induces large negative pressure fields that "suction" thermal energy from the surface, as well as enhances eddy vortex production. The rates of heat flux modeled here, however, are limited only by the thermal diffusion boundary layer, rather than any biological mechanism whose reaction kinetics may slow rates of mass transfer [169]. Field measurements of nutrient uptake rates 22, 170 have shown that the scalar exchange processes occur at maximal levels, indicating these systems are "mass-transfer limited" and thus, the heat transfer results can likely be extended to estimate mass transfer rates, using the appropriate scaling of thermal to scalar diffusivity given in Chapter 5. The numerical modeling undertaken here also has the advantage of avoiding some of the limitations of gypsum dissolution methodology, for example, the effect of changing surface structure as the gypsum model dissolves and the limited efficacy of comparisons across different flow environments [171]. Lastly, numerical modeling provides an easy, low-cost, time-effective strategy to investigate a range of parameterizations of flow conditions and surface morphologies.

In addition to considerations of mass flux on coral health, the dynamics of the thermal boundary layer and the rate of heat flux play vital roles in mitigating thermal stress and associated photoinhibition of zooxanthellae and bleaching events 172 . Hemispherical stony corals have been shown to be more susceptible to surface warming compared to branching corals 32 and those with larger aspect ratios, i.e., greater heightto-diameter ratios, were reported to suffer greater mortality during thermal stress events 173. Micro-profiles of the thermal boundary layer across coral surfaces with "uneven topography" revealed fine-scale variations and patchiness 162 — a finding consistent with the hydrodynamic PIV results presented here in Chapter 3 and the modeling of temperature profiles in Chapter 5. Though coral thermal flux has been shown to depend on flow speed and surface topography, no careful classification of coral roughness type and its effect on heat transfer has been undertaken until this dissertation. The idealized roughness models used in Chapters 4 and 5 were designed with engineering analogues, i.e., two-dimensional bar roughness, as a simplified representation of coral surface morphology in order to isolate the influence of roughness spacingto-height ratios on hydrodynamics and heat transfer. However, the lack of fidelity of the idealized models to 
actual coral morphology raises the question: can the results presented in this dissertation yield insights into real coral biophysics?

Two key features of the heat transfer data presented in Chapter 5 allow us to connect the idealized model results to potential implications for actual corals in the environment. First, the underlying physics that was shown to affect optimization trade-offs for the simplified models - for example, the finding that under certain flow conditions (i.e., fast, wavy flows) total surface area becomes a more important driver of heat transfer than roughness-generated mixing - applies equally well to real corals with different morphologies, though with different specific trade-offs for their more complex morphology. Second, the inclusion of an actual coral skeleton in the numerical modeling in Chapter 5 provides us with an indication of the scale of heat transfer enhancement resulting from the additional variation in surface topography. As the $w / k$ roughness ratio is increased from 0.5 to 2.0 , the heat transfer coefficient increases between $40-90 \%$ depending on flow condition. The coral model has a $w / k$ ratio between the d-type and k-type models-approximately equal to 1.0 - but the average heat transfer coefficient is $20-40 \%$ larger than the k-type model. Thus, we would expect the trend of increasing rates of heat transfer with increasing roughness to extend to real corals, but with an additional enhancement to account for the added complexity in surface topography.

\subsection{Relevance to Engineering Literature}

Despite the biological motivation underpinning the main questions of this dissertation, the findings also contribute new results to the physics and engineering literature, particularly with respect to flow and heat transfer characteristics over bluff bodies with roughness. Though the investigated parameter ranges for Reynolds number and roughness ratios were not as broad as are available in the literature, the hydrodynamic and heat transfer findings are largely consistent with previous work, particularly with regard to polar separation angles, form drag relationships, and convection coefficients. The results demonstrate that trade-offs exist between maximizing surface area by densely packing roughness elements together versus maximizing drag and turbulence production by spacing roughness elements broadly apart - a physical consideration that has been shown to be inherent to flat plate roughness as well. In general, heat transfer rates peak when resistance to the flow is maximized, and for flat plate two-dimensional bar roughness, this corresponds to a peak in form drag [138 and the greatest strength of outward fluid ejection from roughness cavities [174]. This typically occurs at $w / k \sim 7-9[138,151$, though differences in rates of heat transfer persist depending on the shape of the roughness elements, even for those that produce identical shifts in roughness functions 175, 176. Such wide spacing is not usually seen in coral morphology —and thus such large $w / k$ ratios were not tested - but this suggests that other biologically driven pressures are contributing to phenotypic devel- 
opment, and importantly, that the wave-driven flows in the ocean environment create different cost-benefit evaluations for roughness types compared to the unidirectional flow typically employed in flat plate roughness studies.

Additionally, the models tested here - and the real hemispherical corals they represent - are not well described by flat plate roughness. The experimental and numerical results presented in Chapters 4 and 5 are the first investigation of rib-type roughness on a wall-mounted hemisphere. Studies of flows past spherical bluff bodies have shown the amount of form drag remains independent of Reynolds number until a critical value is reached (approximately $R e_{c r i t}=4 \times 10^{6}$ ), above which the previously laminar boundary layer flow transitions to turbulent flow and a smaller drag value is established [112. The presence of surface roughness accelerates this transition, with larger roughness reducing the value of $R e_{\text {crit }}$ more than smaller roughness and also creating a larger supercritical drag for $R e>R e_{\text {crit }}$ 85. Though such large Reynolds numbers were not investigated, the hydrodynamic results are consistent with these previous findings, and the PIV results of Chapter 4 showed that the k-type roughness accelerates this transition to turbulence (and thus increases the separation angle) at a Reynolds number much lower than has been seen for sand-type roughness or small spherical protuberances 85 . Despite the lower drag at high Reynolds number, the production of velocity fluctuations by the turbulent flow enhances heat transfer rates compared to the laminar regime [152], and the results of the heat transfer simulations are consistent with this finding. What has not been previously demonstrated, however, is that the large-scale bluff body curvature alters the traditional balance of frictional drag to pressure drag for rib roughness, which allows for d-type roughness to outperform k-type roughness in terms of total heat flux under specific flow conditions.

In particular, it is the presence of oscillatory flow that creates the advantage for the larger surface area of the d-type roughness. However, the nature of the oscillatory flow, specifically the wave period $T$ and maximum velocity $u_{\max }$, determines the degree to which this mechanism alters trade-offs, as has been shown in gypsum dissolution experiments where higher wave frequencies enhance mass transfer [64]. The ratio of wave excursion length to object diameter $D$ defines the dimensionless Keulegan-Carpenter number, given as $K C=\left(u_{\max } T\right) / D$, and which describes the relative influence of inertial to drag forces. For the high and low oscillatory regimes studied here, the flow conditions give $K C=2.6$ and $K C=7.8$, respectively, which fall on either side of the critical $K C$ number that identifies the onset of vortex shedding, i.e., $\sim 5$ for a smooth surface 177. The role of vortex shedding described in Chapter 5 is an important component in the inertia-driven mechanism that enhances heat transfer under oscillatory forcing. Characterizing the roughness-to-surface area trade-offs that maximize heat transfer for a wall-mounted hemisphere with rib roughness specifically under oscillatory flows have not previously been performed, and this dissertation contributes significant novel findings to the engineering literature, which also helps us better understand the role of coral morphology 
and the ecological consequences that derive from the interaction of flow with coral structures.

Ultimately, the health and sustainability of corals face a host of environmental challenges in the future, of which two of the major threats include excessive nutrient loading and rising sea surface temperatures. Eutrophication promotes algal overgrowth, which has been shown in Chapter 3 to fundamentally alter hydrodynamic characteristics and negatively impact coral mass transfer. Mitigating thermal stress and promoting post-bleaching recovery can be guided by better a understanding of heat transfer processes that are affected by flow conditions and coral morphology, which Chapters 4 and 5 have demonstrated depend on trade-offs between total surface area and roughness-induced turbulence production. In sum, the dissertation provides new insights into the effects on scalar flux resulting from flow interaction with variation in coral morphology and elucidates the underlying physics that govern these processes. It is hoped that understanding these bio-physical interactions can guide policy and management decisions to identify vulnerable areas and target protection for susceptible populations as a way to promote coral resilience and support these fragile organisms and their unique reef ecosystems. 
Appendices 



\section{Appendix A}

\section{Appendix}

\section{A.1 Wave-turbulence decomposition}

The goal of the Phase method is to isolate the turbulent Reynolds stress $\overline{u^{\prime} w^{\prime}}$ from the wave stress $\widetilde{\widetilde{u}} \widetilde{w}$ using spectral sums in frequency space. The wave stress is defined as

$$
\overline{\widetilde{u} \widetilde{w}}=\int_{-f_{N y q u i s t}}^{f_{N y q u i s t}} S_{\widetilde{u} \widetilde{w}}(f) \mathrm{d} f
$$

where $S_{\widetilde{u} \widetilde{w}}$ is the two-sided cross-spectral density (CSD) of the wave orbital velocities. The turbulence spectrum is then defined as the difference between the spectrum of raw velocities (minus the mean flow) and the spectrum of wave orbital velocities

$$
S_{u^{\prime} w^{\prime}}(f)=S_{u w}(f)-S_{\widetilde{w} \widetilde{w}}(f)
$$

Integrating the CSDs in equation A.2 yields an estimate of turbulent Reynolds stress

$$
\overline{u^{\prime} w^{\prime}}=\overline{u w}-\overline{\widetilde{u} \widetilde{w}}
$$

In the spectral domain, the wave orbital frequencies and the turbulence frequencies typically overlap so that the wave-containing frequencies cannot be band-passed out without losing turbulence stress values. Thus, to isolate the contribution of wave stress to the total CSD for the wave-containing frequencies, it is necessary to interpolate the turbulence spectrum beneath the wave peak via a least-squares fit to the theoretical $-5 / 3$ inertial subrange using frequencies outside the wave peak. As the real part of the CSD 
contains both positive and negative values, interpolation is only possible by redefining the CSD in terms of the autospectral densities (PSD) of $u$ and $w$, which contain only positive values. The PSD of $u$ is given by

$$
S_{u u, j}=\frac{1}{\mathrm{~d} f}\left|U_{j}\right|^{2}
$$

where $U_{j}$ is the Fourier transform of $u(t)$ for frequency $f_{j}$. A similar expression gives the PSD for $w$.

For a finite data series, equation A.1 becomes

$$
\overline{\widetilde{u} \widetilde{w}}=\sum_{-N / 2}^{N / 2} \widetilde{U}_{j} * \widetilde{W}_{j}
$$

where $N$ is the number of data points in the Fourier transform, $\widetilde{U}_{j}$ is the Fourier transform of $\widetilde{u}$ at frequency $f_{j}$, and $\widetilde{W}_{j}$ is the Fourier transform of $\widetilde{w}$ at frequency $f_{j}$. The phase of $\widetilde{U}_{j}$ is determined from the Fourier

coefficients of the PSD, and the magnitude of $\widetilde{U}_{j}$ is the difference between the raw $U_{j}$ and the turbulence $U_{j}^{\prime}$, found using the least-squares fit interpolation (figure 3.5 in main text). The turbulent Reynolds stresses are then found through equation A.3.

The Phase method assumes no interaction occurs between the turbulence-induced strain field and the wave-induced strain field, which can "stretch" turbulence and bias Reynolds stress estimates 178. The extent of strain interaction for each measurement record was calculated using the rapidity parameter, $R=(\partial u / \partial z)_{\text {wave }} /(\partial u / \partial z)_{\text {turbulence }}$, which describes the ratio of wave-to-turbulence strain and confirms no interaction if $R \ll 1[179$. All bursts used in the analysis met this criterion.

\section{A.2 Turbulent kinetic energy (TKE) budget equations}

Calculation of $q^{2}$ requires all three velocity components; however, particle image velocimetry (PIV) data only includes horizontal and vertical components. Therefore, we estimated TKE as $q^{2}=0.5\left(\overline{u^{\prime} u^{\prime}}+\right.$ $2.3 \overline{w^{\prime} w^{\prime}}$, where the scaling factor of 2.3 was chosen because shear layers have been shown to possess similar relative intensities of velocity components with $\overline{v^{\prime} v^{\prime}}=1.3 \overline{w^{\prime} w^{\prime}}$ across a range of flow conditions 80 . Wake production $P_{w}$ was estimated using the canopy drag coefficient $C_{d}$ according to $P_{w}=(1 / 2) C_{d} a \bar{u}^{3}$, where $a$ is the frontal area per canopy volume, i.e., canopy density $\lambda$ divided by canopy height $h . C_{d}$ is a function of mean flow velocity $\bar{u}$, algal stem diameter $d$, and the element Reynolds number $R e_{d} \equiv \frac{\bar{u} d}{\nu}$, and is given by $C_{d}=\frac{2 \nu\left(5 R e_{d}^{1 / 3}+0.5 R e_{d}\right)}{\bar{u} d} 180$.

Dissipation estimates were made using the inertial dissipation method, in which the one-dimensional autocorrelation power spectral density (PSD) for vertical fluctuations is fit to a theoretical wavenumber 
Kolmogorov spectrum. For each measurement record, a PSD was generated by ensemble averaging 11 independent spectra created by dividing the 10-min burst interval into Hanning-windowed segments of 2048 data points with $50 \%$ overlap. The ensemble-averaged spectra exhibited a characteristic $-5 / 3$ slope outside the wave-containing frequencies, which indicates the presence of an inertial subrange (see figure 3.5 in main text). Velocity spectra were fit to the model representation given by

$$
S_{w w}(\omega)=\frac{12}{55} \alpha \epsilon^{2 / 3} \bar{u} \omega^{-5 / 3} I(\sigma / \bar{u}, \theta)
$$

where $\alpha=1.5$ is the Kolmogorov constant, $\omega=2 \pi f$ is the PSD radian frequency, and $I(\sigma / \bar{u}, \theta)$ is a function describing the variance in wave-induced horizontal velocity $\sigma^{2}$, the mean current $\bar{u}$, and the angle between waves and current $\theta[181 . I(\sigma / \bar{u}, \theta)$ accounts for convection of turbulence by a spectral wave field 182 and is defined in the appendix of (Trowbridge and Elgar 2001) [181]. To convert frequency spectra into wavenumber space, we employed Taylors "frozen" turbulence approximation, which assumes advection of the turbulence field past the sensor at a rate much faster than turbulent eddy evolution. Taylors hypothesis, $k=2 \pi f / u_{\mathrm{rms}}$, is considered valid when $u^{\prime} / u_{\text {textrmrms }} \ll 1[183$, where the use of root mean square (rms) velocity rather than mean velocity is the more appropriate scale in the presence of waves [184. The magnitude of $S_{w w}$ was then used in equation A.6 to solve for $\epsilon$. Turbulent wake production in the canopy, however, is very fine-scale and bypasses the inertial cascade, which means the IDM method fails to account for this dissipation 78 . Thus, estimates of $\epsilon$ in the canopy are set equal to the negative of wake production, which accounts for the in-canopy balance. 


\section{References}

1. Darwin, C. The Structure and Distribution of Coral Reefs (Smith Elder, London, England, 1842).

2. Odum, H. T. \& Odum, E. P. Trophic structure and productivity of a windward coral reef community on Eniwetok Atoll. Ecological Monographs 25, 291-320 (July 1955).

3. Copper, P. Ancient reef ecosystem expansion and collapse. Coral Reefs 13, 3-11 (Jan. 1994).

4. McAllister, D. E. What is the status of the world's coral reef fishes? Sea Wind 5, 14-18 (1991).

5. Spalding, M. D. \& Grenfell, A. M. New estimates of global and regional coral reef areas. Coral Reefs 16, 225-230 (1997).

6. Veron, J. Corals of the World (Australian Institue of Marine Sciences and CRR Qld Pty Ltd., Australia, 2000).

7. Carté, B. K. Biomedical potential of marine natural products. BioScience 46, 271-286 (Apr. 1996).

8. Cesar, H. Economic analysis of Indonesian coral reefs (Environmentally Sustainable Development Vice Presidency, World Bank, 1996).

9. Moberg, F. \& Folke, C. Ecological goods and services of coral reef ecosystems. Ecological Economics 29, 215-233 (1999).

10. Costanza, R. et al. The value of the world's ecosystem services and natural capital. Nature 387, 253260 (1997).

11. Hoegh-Guldberg, O. et al. Coral reefs under rapid climate change and ocean acidification. Science 318, 1737-1742 (Dec. 2007).

12. Hughes, T. et al. Climate change, human impacts, and the resilience of coral reefs. Science 301, 929$933(2003)$.

13. Hallock, P. \& Schlager, W. Nutrient excess and the demise of coral reefs and carbonate platforms. PALAIOS 1, 389 (Aug. 1986). 
14. Kaandorp, J., Lowe, C., Frenkel, D. \& Sloot, P. Effect of nutrient diffusion and flow on coral morphology. Physical Review Letters 77, 2328-2331 (1996).

15. Goreau, T. Mass expulsion of zooxanthellae from Jamaican reef communities after hurricane flora. Science 145, 383-386 (1964).

16. Hoegh-Guldberg, O \& Smith, G. The effect of sudden changes in temperature, light and salinity on the population density and export of zooxanthellae from the reef corals Stylophora pistillata Esper and Seriatopora hystrix Dana. Journal of Experimental Marine Biology and Ecology 129, 279-303 (Aug. 1989).

17. McCook, L., Jompa, J. \& Diaz-Pulido, G. Competition between corals and algae on coral reefs: a review of evidence and mechanisms. Coral Reefs 19, 400-417 (2001).

18. Sammarco, P. W. Diadema and its relationship to coral spat mortality: Grazing, competition, and biological disturbance. Journal of Experimental Marine Biology and Ecology 45, 245-272 (1980).

19. Miller, M. W. Corallivorous snail removal: evaluation of impact on Acropora palmata. Coral Reefs 19, 293-295 (2001).

20. Patterson, K. L. et al. The etiology of white pox, a lethal disease of the Caribbean elkhorn coral, Acropora palmata. Proceedings of the National Academy of Sciences 99, 8725-8730 (2002).

21. Richmond, R. H. Coral reefs: Present problems and future concerns resulting from anthropogenic disturbance. American Zoologist 33, 524-536 (Jan. 1993).

22. Falter, J. L., Atkinson, M. J. \& Merrifield, M. A. Mass-transfer limitation of nutrient uptake by a wave-dominated reef flat community. Limnology and Oceanography 49(5), 1820-1831 (2004).

23. Lowe, R. J. et al. Spectral wave dissipation over a barrier coral reef. Journal of Geophysical ResearchOceans 110 (2005).

24. Finelli, C. M., Helmuth, B. S. T., Pentcheff, N. D. \& Wethey, D. S. Water flow influences oxygen transport and photosynthetic efficiency in corals. Coral Reefs 25, 47-57 (Mar. 2006).

25. Shapiro, O. H. et al. Vortical ciliary flows actively enhance mass transport in reef corals. Proceedings of the National Academy of Sciences 111, 13391-13396 (2014).

26. Hearn, C. J., Atkinson, M. \& Falter, J. A physical derivation of nutrient-uptake rates in coral reefs: effects of roughness and waves. Coral reefs 20, 347-356 (2001).

27. Bilger, R. W. \& Atkinson, M. J. Anomalous mass transfer of phosphate on coral reef flats. Limnology and Oceanography 37(2), 261-272 (1992). 
28. Atkinson, M. \& Bilger, R. Effects of water velocity on phosphate uptake in coral reef-flat communities. Limnology and Oceanography 37(2), 273-279 (1992).

29. Koehl, M. A. R., Strother, J., Reidenbach, M., Koseff, J. \& Hadfield, M. Individual-based model of larval transport to coral reefs in turbulent, wave-driven flow: behavioral responses to dissolved settlement inducer. Marine Ecology Progress Series 335, 1-18 (2007).

30. Dennison, W. \& Barnes, D. Effect of water motion on coral photosynthesis and calcification. Journal of Experimental Marine Biology and Ecology 115, 67-77 (1988).

31. Mass, T., Genin, A., Shavit, U., Grinstein, M. \& Tchernov, D. Flow enhances photosynthesis in marine benthic autotrophs by increasing the efflux of oxygen from the organism to the water. Proceedings of the National Academy of Sciences 107, 2527-2531 (2010).

32. Jimenez, I. M., Kühl, M., Larkum, A. W. D. \& Ralph, P. J. Heat budget and thermal microenvironment of shallow-water corals: Do massive corals get warmer than branching corals? Limnology and Oceanography 53(4), 1548-1561 (2008).

33. Reidenbach, M. A., Koseff, J. R. \& Monismith, S. G. Laboratory experiments of fine-scale mixing and mass transport within a coral canopy. Physics of Fluids 19 (2007).

34. Chang, S., Elkins, C., Alley, M., Eaton, J. \& Monismith, S. Flow inside a coral colony measured using magnetic resonance velocimetry. Limnology and Oceanography 54, 1819-1827 (2009).

35. Loya, Y et al. Coral bleaching: the winners and the losers. Ecology Letters 4, 122-131 (2001).

36. Luhar, M., Coutu, S., Infantes, E., Fox, S. \& Nepf, H. Wave-induced velocities inside a model seagrass bed. Journal of Geophysical Research: Oceans 115 (2010).

37. Falter, J. L., Atkinson, M. J. \& Coimbra, C. F. Effects of surface roughness and oscillatory flow on the dissolution of plaster forms: Evidence for nutrient mass transfer to coral reef communities. Limnology and Oceanography 50, 246-254 (2005).

38. Leonardi, S., Orlandi, P. \& Antonia, R. A. Properties of d- and k-type roughness in a turbulent channel flow. Physics of Fluids 19, 125101 (2007).

39. Carpenter, R. C. \& Williams, S. L. Effects of algal turf canopy height and microscale substratum topography of profiles of flow speed in a coral forereef environment. Limnology and Oceanography 38(3), 687-694 (1993).

40. Heyward, A. \& Babcock, R. Self-and cross-fertilization in Scleractinian corals. Marine Biology 90, 191-195 (1986). 
41. Heyward, A. \& Negri, A. Natural inducers for coral larval metamorphosis. Coral Reefs 18, 273-279 (1999).

42. Shashar, N., Kinane, S., Jokiel, P. L. \& Patterson, M. R. Hydromechanical boundary layers over a coral reef. Journal of Experimental Marine Biology and Ecology 199, 17-28 (1996).

43. Cloern, J. E. Does the benthos control phytoplankton biomass in South San Francisco Bay? Marine Ecology Progress Series 9, 191-202 (1982).

44. Nichols, F. H. Increased benthic grazing: an alternative explanation for low phytoplankton biomass in northern San Francisco Bay during the 1976-1977 drought. Estuarine, Coastal and Shelf Science 21, 379-388 (1985).

45. Fréchette, M., Butman, C. A. \& Geyer, W. R. The importance of boundary-layer flows in supplying phytoplankton to the benthic suspension feeder, Mytilus edulis L. Limnology and Oceanography 34, 19-36 (1989).

46. O'Riordan, C. A., Monismith, S. G. \& Koseff, J. R. A study of concentration boundary-layer formation over a bed of model bivalves. Limnology and Oceanography 38, 1712-1729 (1993).

47. Jørgensen, B. B. \& Revsbech, N. P. Diffusive boundary layers and the oxygen uptake of sediments and detritus. Limnology and Oceanography 30, 111-122 (1985).

48. Jørgensen, B. B. \& Des Marais, D. J. The diffusive boundary layer of sediments: Oxygen microgradients over a microbial mat. Limnology and Oceanography 35, 1343-1355 (1990).

49. Hondzo, M. Dissolved oxygen transfer at the sediment-water interface in a turbulent flow. Water Resources Research 34, 3525-3533 (1998).

50. Wüest, A. \& Lorke, A. Small-scale hydrodynamics in lakes. Annual Review of Fluid Mechanics 35, 373-412 (2003).

51. Reidenbach, M., Limm, M., Hondzo, M. \& Stacey, M. The effects of bed roughness on boundary layer mixing and mass flux across the sediment-water interface. Water Resources Research 46 (2010).

52. Lorke, A., Müller, B., Maerki, M. \& Wüest, A. Breathing sediments: The control of diffusive transport across the sediment-water interface by periodic boundary-layer turbulence. Limnology and Oceanography 48, 2077-2085 (2003).

53. Røy, H., Hüttel, M. \& Jørgensen, B. The role of small-scale sediment topography for oxygen flux across the diffusive boundary layer. Limnology and Oceanography 47, 837-847 (2002). 
54. Carpenter, R. C., Hackney, J. M. \& Adey, W. H. Measurements of primary productivity and nitrogenase activity of coral reef algae in a chamber incorporating oscillatory flow. Limnology and Oceanography 36, 40-49 (1991).

55. Carpenter, R. C. Competition among marine macroalgae: A physiological perspective. Journal of Phycology 26, 6-12 (Mar. 1990).

56. Jompa, J. \& McCook, L. J. The effects of nutrients and herbivory on competition between a hard coral (Porites cylindrica) and a brown alga (Lobophora variegata). Limnology and Oceanography 47, $527-534(2002)$.

57. Fricke, A., Teichberg, M., Beilfuss, S. \& Bischof, K. Succession patterns in algal turf vegetation on a Caribbean coral reef. Botanica Marina 54, 111-126 (2011).

58. Diaz-Pulido, G. \& McCook, L. J. The fate of bleached corals: patterns and dynamics of algal recruitment. Marine Ecology Progress Series 232, 115-128 (2002).

59. De Nys, R., Coll, J. \& Price, I. Chemically mediated interactions between the red alga Plocamium hamatum (Rhodophyta) and the octocoral Sinularia cruciata (Alcyonacea). Marine Biology 108, 315320 (June 1991).

60. Littler, M. M. \& Littler, D. S. Epithallus sloughing: a self-cleaning mechanism for coralline algae. Coral Reefs 18, 204-204 (1999).

61. Kundu, P. K. \& Cohen, I. M. Fluid Mechanics Fourth Edition (Academic Press, USA, 2008).

62. Atkinson, M., Falter, J. \& Hearn, C. J. Nutrient dynamics in the Biosphere 2 coral reef mesocosm: water velocity controls $\mathrm{NH}_{4}$ and $\mathrm{PO}_{4}$ uptake. Coral Reefs 20, 341-346 (2001).

63. Lowe, R., Koseff, J. R. \& Monismith, S. G. Oscillatory flow through submerged canopies: Part 1. Velocity structure. Journal of Geophysical Research 110 (2005).

64. Reidenbach, M., Koseff, J. R., Monismith, S. G., Steinbuck, J. \& Genin, A. The effects of waves and morphology on mass transfer within branched reef corals. Limnology and Oceanography 51, 1134-1141 (2006).

65. Fredsøe, J. Turbulent boundary layer in wave-current motion. Journal of Hydraulic Engineering 110, 1103-1120 (1984).

66. Trowbridge, J. H. On a technique for measurement of turbulent shear stress in the presence of surface waves. Journal of Atmospheric and Oceanic Technology 15, 290-298 (1998).

67. Grant, W. D. \& Madsen, O. S. Combined wave and current interaction with a rough bottom. Journal of Geophysical Research 84, 1797-1808 (1979). 
68. Hino, M., Kashiwayanagi, M., Nakayama, A. \& Hara, T. Experiments on the turbulence statistics and the structure of a reciprocating oscillatory flow. Journal of Fluid Mechanics 131, 363-400 (1983).

69. Sleath, J. Turbulent oscillatory flow over rough beds. Journal of Fluid Mechanics 182, 369-409 (1987).

70. Dean, R. G. \& Dalrymple, R. A. Water Wave Mechanics for Engineers and Scientists (World Scientific, Singapore, 1991).

71. Jonsson, I. G. Measurements in the turbulent wave boundary layer. 10th Congress I.A.H.R. 1 (1963).

72. Bakker, W. T. \& Van Doorn, T. Near-bottom velocities in waves with a current. Coastal Engineering Proceedings 1 (1978).

73. Smith, J. D. Modeling of sediment transport on continental shelves. In: The sea 6 (ed Goldberg, E. D.) 539-577 (1977).

74. Jensen, B., Sumer, B. \& Fredsøe, J. Turbulent oscillatory boundary layers at high Reynolds numbers. Journal of Fluid Mechanics 206, 265-297 (1989).

75. Hansen, J. \& Reidenbach, M. Wave and tidally driven flows in eelgrass beds and their effect on sediment suspension. Marine Ecology Progress Series 448, 271-287 (2012).

76. Rosman, J., Koseff, J., Monismith, S. G. \& Grover, J. A field investigation into the effects of a kelp forest (Macrocystis pyrifera) on coastal hydrodynamics and transport. Journal of Geophysical Research 112 (2007).

77. Lowe, R. J., Shavit, U., Falter, J. L., Koseff, J. R. \& Monismith, S. G. Modeling flow in coral communities with and without waves: A synthesis of porous media and canopy flow approaches. Limnology and Oceanography 53, 2668 (2008).

78. Finnigan, J. Turbulence in plant canopies. Annual Review of Fluid Mechanics 32, 519-571 (2000).

79. Nepf, H. M. Flow and transport in regions with aquatic vegetation. Annual Review of Fluid Mechanics 44, 123-142 (2012).

80. Raupach, M., Antonia, R. \& Rajagopalan, S. Rough-wall turbulent boundary layers. Applied Mechanical Review 44, 1-25 (1991).

81. Ghisalberti, M. \& Nepf, H. The structure of the shear layer in flows over rigid and flexible canopies. Environmental Fluid Mechanics 6, 277-301 (2006).

82. Brown, A. L. \& Carpenter, R. C. Water flow influences the mechanisms and outcomes of interactions between massive Porites and coral reef algae. Marine Biology (Dec. 2014). 
83. Raupach, M., Finnigan, J. \& Brunet, Y. Coherent eddies and turbulence in vegetation canopies: The mixing-layer analogy. Boundary-Layer Meteorology 60, 375-395 (1996).

84. Meyers, T. P. \& Baldocchi, D. D. The budgets of turbulent kinetic energy and Reynolds stress within and above a deciduous forest. Agricultural and Forest Meteorology 53, 207-222 (1991).

85. Achenbach, E. The effects of surface roughness and tunnel blockage on the flow past spheres. Journal of Fluid Mechanics 65, 113-125 (1974).

86. Choi, J., Jeon, W.-P. \& Choi, H. Mechanism of drag reduction by dimples on a sphere. Physics of Fluids 18 (2006).

87. Perry, A. E., Schofield, W. H. \& Joubert, P. N. Rough wall turbulent boundary layers. Journal of Fluid Mechanics 37, 383-413 (1969).

88. Jimenez, J. Turbulent flows over rough walls. Annual Review of Fluid Mechanics 36, 173-196 (2004).

89. Nikuradse, J. Laws of flow in rough pipes. National Advisory Committee for Aeronautics. Tech. Mem. 1292, 60 (1933).

90. Kays, W. M. Turbulent Prandtl number-where are we? Journal of Heat Transfer 116, 284-295 (1994).

91. Baird, M. E. \& Atkinson, M. Measurement and prediction of mass transfer to experimental coral reef communities. Limnology and Oceanography 42(8), 1685-1693 (1997).

92. O'Connor, B. \& Hondzo, M. Dissolved oxygen transfer to sediments by sweep and eject motions in aquatic environments. Limnology and Oceanography 53(2), 566-578 (2008).

93. Incropera, F. P. \& DeWitt, D. P. Fundamentals of Heat and Mass Transfer, Fourth Edition (John Wiley and Sons, New York, NY, 1996).

94. Stocking, J. B., Rippe, J. P. \& Reidenbach, M. A. Structure and dynamics of turbulent boundary layer flow over healthy and algae-covered corals. Coral Reefs 35, 1047-1059 (2016).

95. Reidenbach, M., Monismith, S. G., Koseff, J. R., Yahel, G. \& Genin, A. Boundary layer turbulence and flow structure over a fringing coral reef. Limnology and Oceanography 51, 1956-1968 (2006).

96. Falter, J. L., Atkinson, M. J., Lowe, R. J., Monismith, S. G. \& Koseff, J. R. Effects of nonlocal turbulence on the mass transfer of dissolved species to reef corals. Limnology and Oceanography 52, $274-285$ (2007).

97. Monismith, S. Hydrodynamics of coral reefs. Annual Review of Fluid Mechanics 39, 37-55 (2007). 
98. Brown, A. \& Carpenter, R. Water-flow mediated oxygen dynamics within massive Porites-algal turf interactions. Marine Ecology Progress Series 490, 1-10 (Sept. 2013).

99. Li, A. \& Reidenbach, M. A. Forecasting decadal changes in sea surface temperatures and coral bleaching within a Caribbean coral reef. Coral Reefs 33, 847-861 (2014).

100. Gross, T. F. \& Nowell, A. R. Mean flow and turbulence scaling in a tidal boundary layer. Continental Shelf Research 2, 109-126 (1983).

101. Bendat, J. \& Piersol, A. Random Data, Analysis and Measurement Proceedures, Second Edition 2nd ed. (ed Wiley-Interscience) (New York, 1986).

102. Nikora, V. et al. Double-averaging concept for rough-bed open-channel and overland flows: Theoretical background. Journal of Hydraulic Engineering 133, 873-883 (2007).

103. Voulgaris, G. \& Trowbridge, J. H. Evaluation of the acoustic Doppler velocimeter (ADV) for turbulence measurements. Journal of Atmospheric and Oceanic Technology 15, 272-289 (1998).

104. Rusello, P. Near boundary measurements with a profiling acoustic Doppler velocimeter. Proceedings of the Hydraulic Measurement and Experimental Methods Conference, ASCE (2012).

105. Sveen, J. in Mechanics and Applied Mathematics (Dept. of Mathematics, University of Oslo, 2004).

106. Reidenbach, M., George, N. \& Koehl, M. A. R. Antennule morphology and flicking kinematics facilitate odor sampling by the spiny lobster, Panulirus argus. Journal of Experimental Biology 211, 2849-2858 (2008).

107. Bricker, J. D. \& Monismith, S. G. Spectral wave-turbulence decomposition. Journal of Atmospheric and Oceanic Technology 24, 1479-1487 (Aug. 2007).

108. Wiberg, P. \& Smith, J. A comparison of field data and theoretical models for wave-current interactions at the bed on the continental shelf. Continental Shelf Research 2, 147-162 (1983).

109. Soulsby, R. L. \& Dyer, K. R. The form of the near-bed velocity profile in a tidally accelerating flow. Journal of Geophysical Research 81, 8067-8073 (1981).

110. Jonsson, I. Wave boundary layers and friction factors. 10th Coastal Engineering Conference. American Society of Civil Engineering (Sept. 1966).

111. Madsen, O. S., Poon, Y.-K. \& Graber, H. C. Spectral wave attenuation by bottom friction: theory. Coastal Engineering Proceedings 1 (1988).

112. Achenbach, E. Experiments on the flow past spheres at very high Reynolds numbers. Journal of Fluid Mechanics 54, 565-575 (1972). 
113. Taneda, S. Visual observations of the flow past a sphere at Reynolds numbers between $10^{4}$ and $10^{6}$. Journal of Fluid Mechanics 85, 187-192 (1978).

114. Johnson, T. A. \& Patel, V. C. Flow past a sphere up to a Reynolds number of 300. Journal of Fluid Mechanics 378, 19-70 (1999).

115. Tomboulides, A. G. \& Orszag, S. A. Numerical investigation of transitional and weak turbulent flow past a sphere. Journal of Fluid Mechanics 416, 45-73 (2000).

116. Sakamoto, H. \& Haniu, H. The formation mechanism and shedding frequency of vortices from a sphere in uniform shear flow. Journal of Fluid Mechanics 287, 151-171 (1995).

117. Taniguchi, S., Sakamoto, H., Kiya, M. \& Arie, M. Time-averaged aerodynamic forces acting on a hemisphere immersed in a turbulent boundary. Journal of Wind Engineering and Industrial Aerodynamics 9, $257-273(1982)$.

118. Laforsch, C. et al. A precise and non-destructive method to calculate the surface area in living scleractinian corals using X-ray computed tomography and 3D modeling. Coral Reefs 27, 811-820 (2008).

119. Cowen, E. \& Monismith, S. A hybrid digital particle tracking velocimetry technique. Experiments in Fluids 22, 199-211 (1997).

120. Moffatt, H. K. Viscous and resistive eddies near a sharp corner. 18, 1-18 (1964).

121. Pope, S. B. Turbulent Flows (Cambridge University Press, Cambridge, 2000).

122. Balachandar, S., Mittal, R. \& Najjar, F. M. Properties of the mean recirculation region in the wakes of two-dimensional bluff bodies. Journal of Fluid Mechanics 351, 167-199 (1997).

123. Hench, J. L. \& Rosman, J. H. Observations of spatial flow patterns at the coral colony scale on a shallow reef flat. Journal of Geophysical Research: Oceans 118, 1142-1156 (Mar. 2013).

124. Fincham, A. M., Maxworthy, T. \& Spedding, G. R. Energy dissipation and vortex structure in freely decaying, stratified grid turbulence. Dynamics of Atmospheres and Oceans 23, 155-169 (1996).

125. Lu, S. \& Willmarth, W. Measurements of the structure of the Reynolds stress in a turbulent boundary layer. Journal of Fluid Mechanics 60, 481-571 (1973).

126. Savory, E \& Toy, N. Hemisphere and hemisphere-cylinders in turbulent boundary layers. Journal of Wind Engineering and Industrial Aerodynamics 23, 345-364 (1986).

127. Clift, R., Grace, J. R. \& Weber, M. E. Bubbles, drops, and particles (Courier Corporation, 2005). 
128. Falter, J. L., Lowe, R. J. \& Zhang, Z. Toward a universal massmomentum transfer relationship for predicting nutrient uptake and metabolite exchange in benthic reef communities. Geophysical Research Letters (2016).

129. Savory, E. \& Toy, N. The flow regime in the turbulent near wake of a hemisphere. Experiments in Fluids 4, 181-188 (1986).

130. Tamai, N., Asaeda, T. \& Tanaka, N. Vortex structures around a hemispheric hump. Boundary-Layer Meteorology 39, 301-314 (1987).

131. Prasad, A. \& Williamson, C. H. K. The instability of the shear layer separating from a bluff body. Journal of Fluid Mechanics 333, 375-402 (1997).

132. Hussain, A. K. M. F. Coherent structures and turbulence. Journal of Fluid Mechanics 173, 303-356 (1986).

133. Ghisalberti, M. \& Nepf, H. Mixing layers and coherent structures in vegetated aquatic flows. Journal of Geophysical Research 107, 3-1-3-11 (2002).

134. Sebens, K. Water flow and coral colony size: interhabitat comparisons of the octocoral Alcyonium siderium. Proceedings of the National Academy of Sciences 81, 5473-5477 (1984).

135. Nakamura, T. \& Van Woesik, R. Water-flow rates and passive diffusion partially explain differential survival of corals during the 1998 bleaching event. Marine Ecology Progress Series 212, 301-304 (2001).

136. Done, T. \& Potts, D. Influences of habitat and natural disturbances on contributions of massive Porites corals to reef communities. Marine Biology 114, 479-493 (1992).

137. Gloss, D. \& Herwig, H. Wall roughness effects in laminar flows: an often ignored though significant issue. Experiments in Fluids 49, 461-470 (2010).

138. Leonardi, S., Orlandi, P., Smalley, R. J., Djenidi, L. \& Antonia, R. A. Direct numerical simulations of turbulent channel flow with transverse square bars on one wall. Journal of Fluid Mechanics 491, 229-238 (2003).

139. Bhagoria, J., Saini, J. \& Solanki, S. Heat transfer coefficient and friction factor correlations for rectangular solar air heater duct having transverse wedge shaped rib roughness on the absorber plate. Renewable Energy 25, 341 -369 (2002).

140. Webb, R., Eckert, E. \& Goldstein, R. Heat transfer and friction in tubes with repeated-rib roughness. International Journal of Heat and Mass Transfer 14, 601 -617 (1971). 
141. Roshko, A. Perspectives on bluff body aerodynamics. Journal of Wind Engineering and Industrial Aerodynamics 49, $79-100$ (1993).

142. Cantwell, B. \& Coles, D. An experimental study of entrainment and transport in the turbulent near wake of a circular cylinder. Journal of Fluid Mechanics 136, 321-374 (1983).

143. Bradshaw, P. Possible origin of Prandt's mixing-length theory. Nature 249, 135-136 (May 1974).

144. Reidenbach, M., Koseff, J. R. \& Koehl, M. A. R. Hydrodynamic forces on larvae affect their settlement on coral reefs in turbulent, wave-driven flow. Limnology and Oceanography 54, 318-330 (2009).

145. Cardinale, B. J., Palmer, M. A. \& Collins, S. L. Species diversity enhances ecosystem functioning through interspecific facilitation. Nature 415, 426-429 (Jan. 2002).

146. Blair, M. F. Influence of free-stream turbulence on turbulent boundary layer heat transfer and mean profile development, Part I-Experimental data. Journal of Heat Transfer 105, 33-40 (Feb. 1983).

147. Dipprey, D. \& Sabersky, R. Heat and momentum transfer in smooth and rough tubes at various Prandtl numbers. International Journal of Heat and Mass Transfer 6, 329 -353 (1963).

148. Hosni, M., Coleman, H. W. \& Taylor, R. P. Measurements and calculations of rough-wall heat transfer in the turbulent boundary layer. International Journal of Heat and Mass Transfer 34, 1067 -1082 (1991).

149. Miyake, Y., Tsujimoto, K. \& Nakaji, M. Direct numerical simulation of rough-wall heat transfer in a turbulent channel flow. International Journal of Heat and Fluid Flow 22, 237 -244 (2001).

150. Aupoix, B. Improved heat transfer predictions on rough surfaces. International Journal of Heat and Fluid Flow 56, 160 -171 (2015).

151. Ryu, D., Choi, D. \& Patel, V. Analysis of turbulent flow in channels roughened by two-dimensional ribs and three-dimensional blocks. Part II: Heat transfer. International Journal of Heat and Fluid Flow 28, $1112-1124(2007)$.

152. Whitaker, S. Forced convection heat transfer correlations for flow in pipes, past flat plates, single cylinders, single spheres, and for flow in packed beds and tube bundles. AIChE Journal 18, 361-371 (1972).

153. Lowe, R., Koseff, J., Monismith, S. G. \& Falter, J. Oscillatory flow through submerged canopies: Part 2. Canopy mass transfer. Journal of Geophysical Research 110 (2005).

154. Nicoud, F. \& Ducros, F. Subgrid-scale stress modelling based on the square of the velocity gradient tensor. Flow, Turbulence and Combustion 62, 183-200 (1999). 
155. Kader, B. Temperature and concentration profiles in fully turbulent boundary layers. International Journal of Heat and Mass Transfer 24, 1541 -1544 (1981).

156. ANSYS CFX-Solver Modeling Guide Release 15.0 (Canonsburg, PA, 2013).

157. Nakamura, I. Steady wake behind a sphere. The Physics of Fluids 19, 5-8 (1976).

158. Dey, S., Sarkar, S., Bose, S. K., Tait, S. \& Castro-Orgaz, O. Wall-wake flows downstream of a sphere placed on a plane rough wall. Journal of Hydraulic Engineering 137, 1173-1189 (2011).

159. Achenbach, E. Vortex shedding from spheres. Journal of Fluid Mechanics 62, 209-221 (1974).

160. Hunt, J. C., Wray, A. A. \& Moin, P. Eddies, streams, and convergence zones in turbulent flows. Center for Turbulence Research Report CTR-S88 (1988).

161. Kaandorp, J. A. et al. Morphogenesis of the branching reef coral Madracis mirabilis. Proceedings of the Royal Society of London B: Biological Sciences 272, 127-133 (2005).

162. Jimenez, I. M., Kuhl, M., Larkum, A. W. D. \& Ralph, P. J. Effects of flow and colony morphology on the thermal boundary layer of corals. Journal of The Royal Society Interface 8, 1785-1795 (2011).

163. Tantirige, S. \& Trass, O. Mass transfer at geometrically dissimilar rough surfaces. The Canadian Journal of Chemical Engineering 62, 490-496 (1984).

164. Flot, J.-F., Magalon, H., Cruaud, C., Couloux, A. \& Tillier, S. Patterns of genetic structure among Hawaiian corals of the genus Pocillopora yield clusters of individuals that are compatible with morphology. Comptes Rendus Biologies 331, 239 -247 (2008).

165. Vollmer, S. V. \& Palumbi, S. R. Hybridization and the Evolution of Reef Coral Diversity. Science 296, 2023-2025 (2002).

166. Mass, T., Brickner, I., Hendy, E. \& Genin, A. Enduring physiological and reproductive benefits of enhanced flow for a stony coral. Limnology and Oceanography 56, 2176-2188 (2011).

167. Thomas, F. I. M. \& Atkinson, M. J. Ammonium uptake by coral reefs: Effects of water velocity and surface roughness on mass transfer. Limnology and Oceanography 42, 81-88 (1997).

168. Nakamura, T., Yamasaki, H. \& van Woesik, R. Water flow facilitates recovery from bleaching in the coral Stylophora pistillata. Marine Ecology Progress Series 256, 287-291 (2003).

169. Sanford, L. \& Crawford, S. Mass transfer versus kinetic control of uptake across solid-water boundaries. Limnology and Oceanography 45(5), 1180-1186 (2000).

170. Atkinson, M. Rates of phosphate uptake by coral reef flat communities. Limnology and Oceanography 32(2), 426-435 (1987). 
171. Porter, E., Sanford, L. \& Suttles, S. Gypsum dissoltuion is not a universal integrator of 'water motion'. Limnology and Oceanography 45(1), 145-158 (2000).

172. Smith, L. W. \& Birkeland, C. Effects of intermittent flow and irradiance level on back reef Porites corals at elevated seawater temperatures. Journal of Experimental Marine Biology and Ecology 341, $282-294(2007)$.

173. Van Woesik, R., Irikawa, A., Anzai, R. \& Nakamura, T. Effects of coral colony morphologies on mass transfer and susceptibility to thermal stress. Coral Reefs 31, 633-639 (2012).

174. Leonardi, S., Orlandi, P., Djenidi, L. \& Antonia, R. Structure of turbulent channel flow with square bars on one wall. International Journal of Heat and Fluid Flow 25. Turbulence and Shear Flow Phenomena (TSFP-3), $384-392$ (2004).

175. Krogstad, P.-Å, Antonia, R. A. \& Browne, L. W. B. Comparison between rough-and smooth-wall turbulent boundary layers. Journal of Fluid Mechanics 245, 599-617 (1992).

176. Krogstadt, P.- $\AA \&$ Antonia, R. A. Surface roughness effects in turbulent boundary layers. Experiments in Fluids 27, 450-460 (1999).

177. Bearman, P. W., Downie, M. J., Graham, J. M. R. \& Obasaju, E. D. Forces on cylinders in viscous oscillatory flow at low Keulegan-Carpenter numbers. Journal of Fluid Mechanics 154, 337-356 (1985).

178. Teixeira, M. \& Belcher, S. On the distortion of turbulence by a progressive surface wave. Journal of Fluid Mechanics 458, 229-267 (2002).

179. Monismith, S. G. \& Magnaudet, J. in Physical Processes in Lakes and Oceans (ed Imberger, J.) 662 (1998).

180. Tanino, Y. \& Nepf, H. M. Laboratory investigation of mean drag in a random array of rigid, emergent cylinders. Journal of Hydraulic Engineering 134, 34-41 (2008).

181. Trowbridge, J. \& Elgar, S. Turbulence measurements in the surf zone. Journal of Physical Oceanography 31 (2001).

182. Lumley, J. L. \& Terray, E. A. Kinematics of turbulence convected by a random wave field. Journal of Physical Oceanography 13, 2000-2007 (1983).

183. Lumley, J. Interpretation of time spectra measured in high-intensity shear flows. The Physics of Fluids 8, 1056-1062 (1965).

184. Agrawal, Y. et al. Enhanced dissipation of kinetic energy beneath surface waves. Nature 359, 219-220 (1992). 